\title{
ON CONFLICT AND RESOLUTION IN THE TERMITE-FUNGUS SYMBIOSIS
}

Sabine M.E. Vreeburg

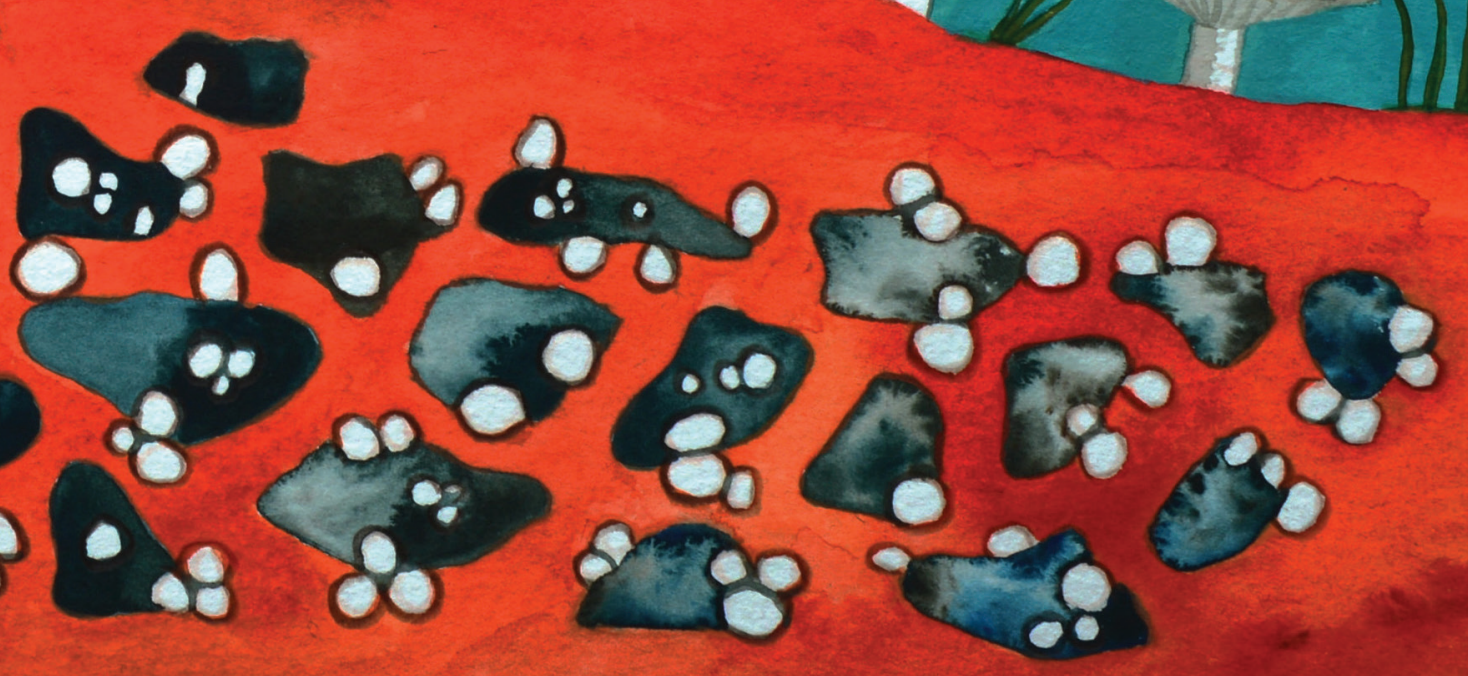




\section{Propositions}

1. The asexual spore balls formed by Termitomyces are not the primordia of mushrooms (this thesis).

2. There is no such thing as a standard life cycle for basidiomycete fungi (this thesis)

3. If the outcome of a student's project is vital to her or his supervisor, a conflict of interest is inevitable.

4. Society is the customer of science.

5. Selection for soundbites and catchy headlines in science communication is in conflict with the credibility of science as a whole.

6. The core issue for the acceptance of evolutionary theory is the question whether evolutionary theory applies to humans.

7. Differences in payed parental-leave drive the observed patterns of paternal and maternal child-care.

8. Men in top positions are often not hired for their qualities, but rather because they are men.

Propositions belonging to the thesis, entitled:

"On conflict and resolution in the termite-fungus symbiosis"

Sabine M.E. Vreeburg

Wageningen, 15 September 2020 


\section{ON CONFLICT AND RESOLUTION IN}

\section{THE TERMITE-FUNGUS}

SYMBIOSIS

Sabine M.E. Vreeburg 


\section{Thesis Committee}

\section{Promotor}

Prof. Dr B. J. Zwaan

Professor of Genetics

Wageningen University \& Research

\section{Co-promotor}

Prof. Dr D.K. Aanen

Laboratory of Genetics

Wageningen University \& Research

\section{Other members}

Prof. Dr F.P.M. Govers, Wageningen University \& Research

Prof. Dr J. Korb, University of Freiburg, Germany

Prof. Dr T.W. Kuyper, Wageningen University \& Research

Dr A.F. van Peer, Wageningen University \& Research

This research was conducted under the auspices of the C.T. de Wit Graduate School for Production Ecology and Resource Conservation. 


\section{ON CONFLICT AND RESOLUTION IN THE TERMITE-FUNGUS SYMBIOSIS}

Sabine Maria Emerentiana Vreeburg

submitted in fulfilment of the requirements for the degree of doctor at Wageningen University by the authority of the Rector Magnificus, Prof. Dr A.P.J. Mol, in the presence of the Thesis Committee appointed by the Academic Board to be defended in public on Tuesday 15 September 2020 at 11 a.m. in the Aula. 
Sabine Maria Emerentiana Vreeburg

On conflict and resolution in the termite-fungus symbiosis

$\mathrm{PhD}$ thesis, Wageningen University, Wageningen, the Netherlands (2020)

With references, with summary in English

ISBN 978-94-6395-469-3

DOI $10.18174 / 527068$ 


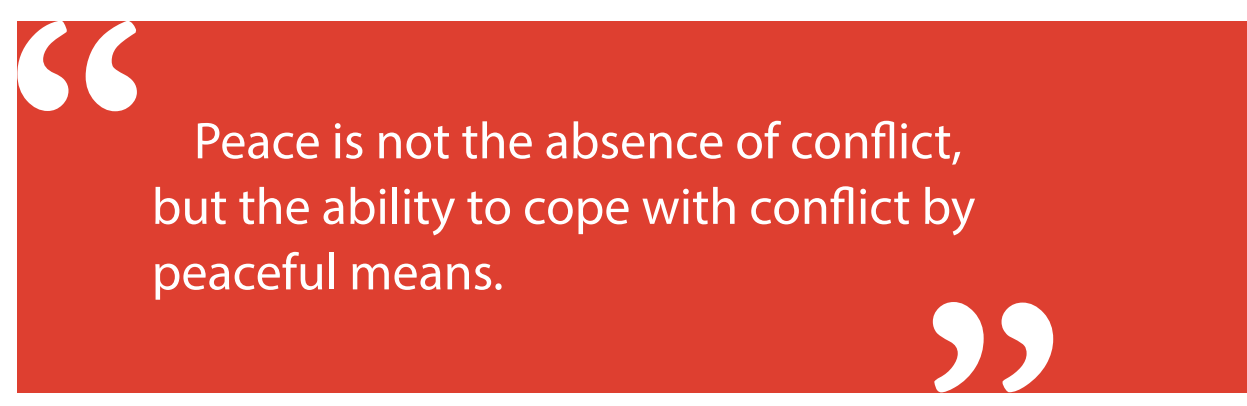

- Ronald Reagan 



\section{Table of Contents}

CHAPTER 1

General introduction

CHAPTER 2

Asexual and sexual reproduction are two separate developmental pathways in a Termitomyces species

CHAPTER 3

Can interaction specificity in the fungus-farming termite symbiosis be explained by nutritional requirements of the fungal crop?

CHAPTER 4

An improved reference assembly and a first linkage map of the Termitomyces symbiont of Macrotermes natalensis

CHAPTER 5

Unholy marriages and eternal triangles - how competition in the mushroom life cycle can lead to genomic conflict

CHAPTER 6

General discussion

REFERENCES

SUMMARY

ACKNOWLEDGEMENTS 


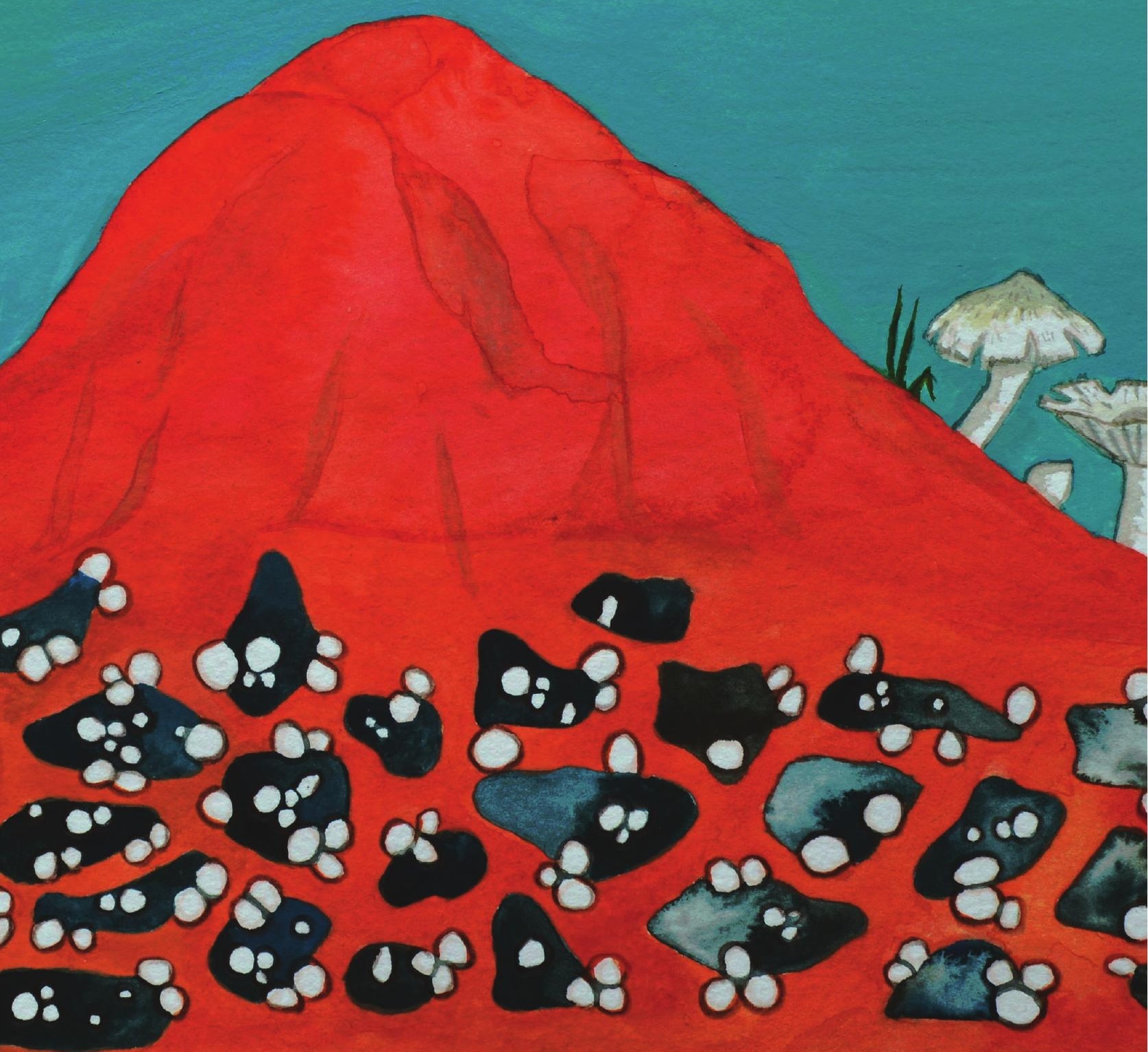




\section{A childhood memory}

"I remember the excitement of hunting for these mushrooms during Christmas summer holidays that we sometimes spent on the farm of a family member in the Otjiwarongo region of Namibia, when I was young. If it rained around Christmas time, which might be some of the very first heavy summer rains after a long dry spell, the mushrooms would pop up around the termite colonies that were scattered through the beautiful savanna of that area (Figure 1). We were holding onto the rails on the back of a slowly moving open truck as we drove through 'the veld', as we call it. You had to look carefully at each mound as you passed, peering through the Acacia thorn bush branches and yellow grass. These mounds are large - up to two meters or taller if I remember correctly - and rock hard. As children we would sometime break one open with a pickaxe and marvel at how hard they were, and at the perfectly smooth large tunnels that ran like veins right through them (Figure 2). Much too large to imagine that it was built, or used, by termites.

You had to spot the mushrooms soon after they emerged, otherwise they would be full of insects and become tough in the sun. But when you did, it was a feast. The species is large, with caps that can be the size of a dinner plate (Figure 1). And there could be 10,20 or 30 of them - maybe the numbers have grown with my fading memory of those days. But there were often many! If you carefully pulled a mushroom by its stem it would bring a long root with it, leaving a hole in the base of the rock-hard mound. We marvelled at how such a soft mushroom body could push through that mound base. Back at home, after washing, we would prepare for a feast. Large specimens could be cut in thick slices and directly fried on the fire, with a bit of salt and butter. There was enough left for butter fried sliced blocks to eat with a meat braai (barbecue) or on bread. The taste is not easy to describe - it is delicious, that is for sure. People say it has a meaty taste - but not quite like meat - much softer, and rich, but not fatty. And with a texture that is firm but melt-inyour mouth mushroom-like. After eating as much as we could, there would still be loads left. The remaining would be sliced into small blocks, lightly fried in butter and frozen for later use in soup or sauces. What a feast! I miss Otjiwarongo, December summer rain, and Termitomyces on the termite mounds!" 


\section{Termitomyces and Termites}

The childhood memory of Professor Bernard Slippers (director of the Forestry and Agricultural Biotechnology Institute (FABI), University of Pretoria, South Africa) paints a picture of the role that the Termitomyces mushrooms play in Africa and Asia. All known Termitomyces mushrooms, the fruits of fungi of the basidiomycete genus Termitomyces, are edible and considered delicacies in the areas where they are found. The mushrooms are picked for personal consumption as well as to be sold on markets, providing a considerable income to local people (Kone et al. 2013). Especially in rural areas, the mushrooms are used as meat and fish replacement in meals (Kone et al. 2013). In addition to their meaty taste and texture, Termitomyces mushrooms are nutritionally a good candidate in the search for alternative protein sources. Termitomyces fungi been shown to contain high amounts of protein (Ogundana and Fagade 1982, Botha and Eicker 1992, Aletor 1995, Kansci et al. 2003, Adejumo and Awosanya 2005, Masamba 2010). Finally, the size of Termitomyces mushrooms can be impressive. Professor Slippers remembers that the species he found in Namibia could be the size of a dinner plate. There is even a species, Termitomyces titanicus, that can reach the size of an umbrella with a diameter up to one meter (Figure 3; Pegler and Piearce 1980).

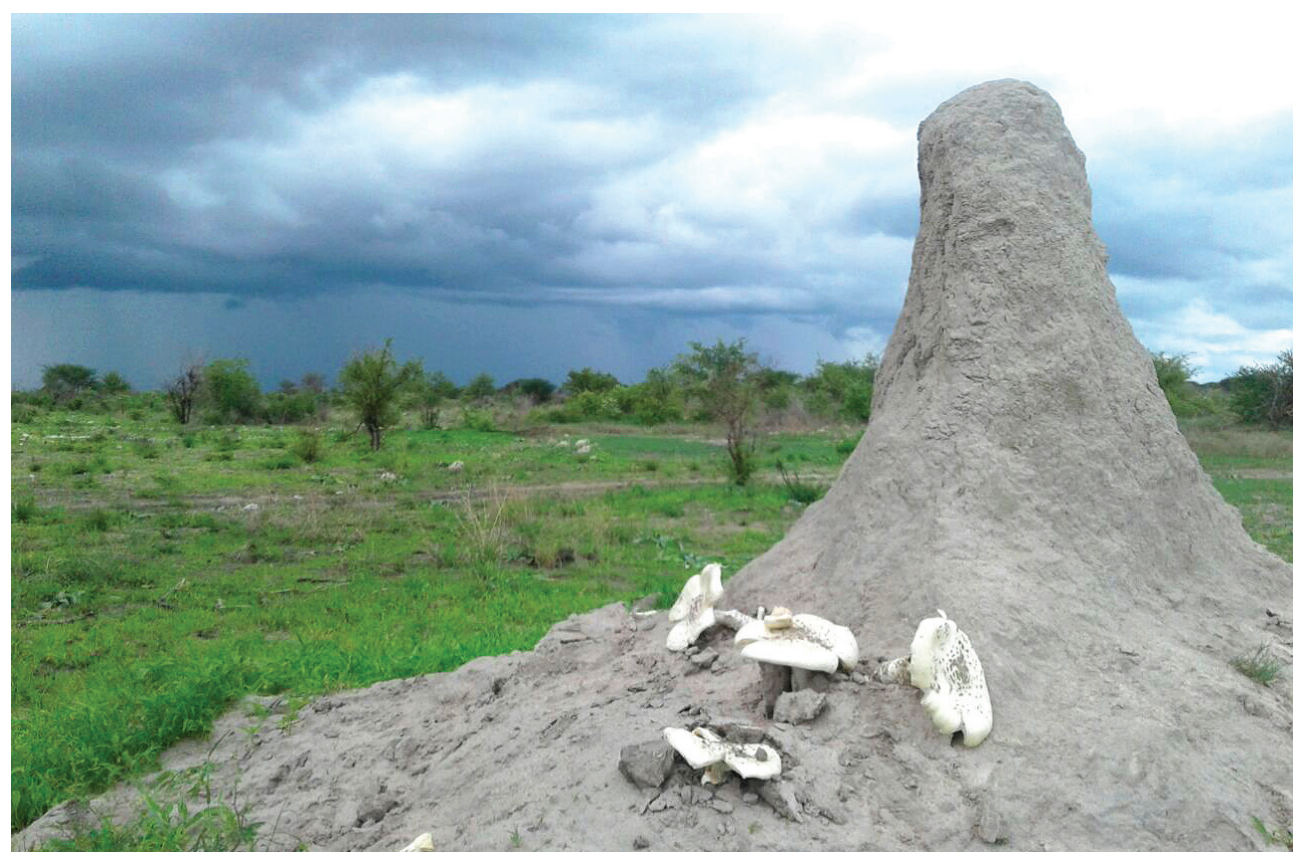

Figure 1| A Macrotermes termite mound in Namibia sprouting Termitomyces mushrooms at the base during the rainy season. "As the grass turns green and the clouds mass for summer downpours, the gargantuan fungi appear as if in celebration of the rains or as manna from the gods." (Gondwana Collection Namibia). Photo credit: (Gondwana Collection Namibia). 
The Termitomyces mushrooms, those that Professor Bernard Slippers so fondly remembers, do not grow on termite mounds by coincidence as was once thought (Petch 1906). Termitomyces fungi - of which the fruits are mushrooms - live in a mutualistic symbiosis with socalled fungus-growing termites (order Isoptera; family Termitidae; subfamily Macrotermitinae). Both partners benefit from being in the symbiosis: the termites provide a constant food supply and optimal growth conditions for the fungi, and in return, the fungi convert complex plant substrates into a more nutritious food source for the termites. Through their cooperation, termites and their fungi have become obligatory dependent on each other, which means that one cannot live without the other (Aanen et al. 2002, FrØSlev et al. 2003).

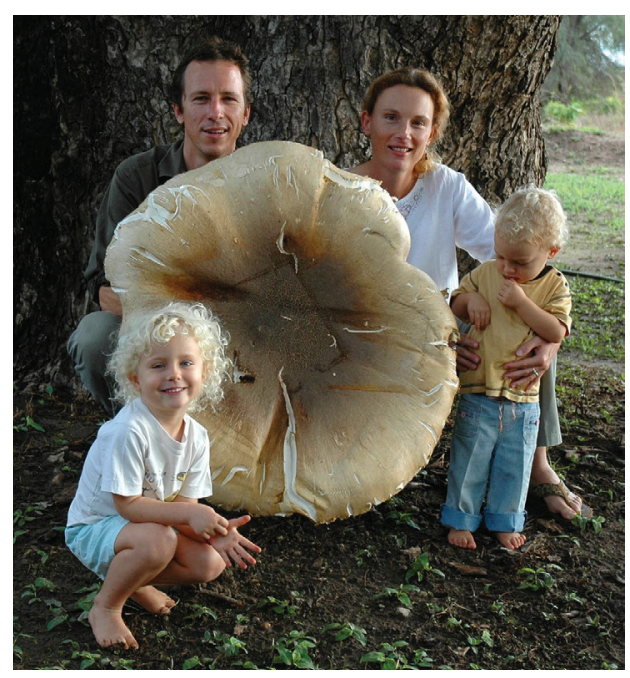

Figure $3 \mid$ The cap of a Termitomyces titanicus mushroom found in Zambia. The Termitomyces titanicus mushroom is likely the largest edible mushroom in the world. Photo credit: Groenendijk (2016).

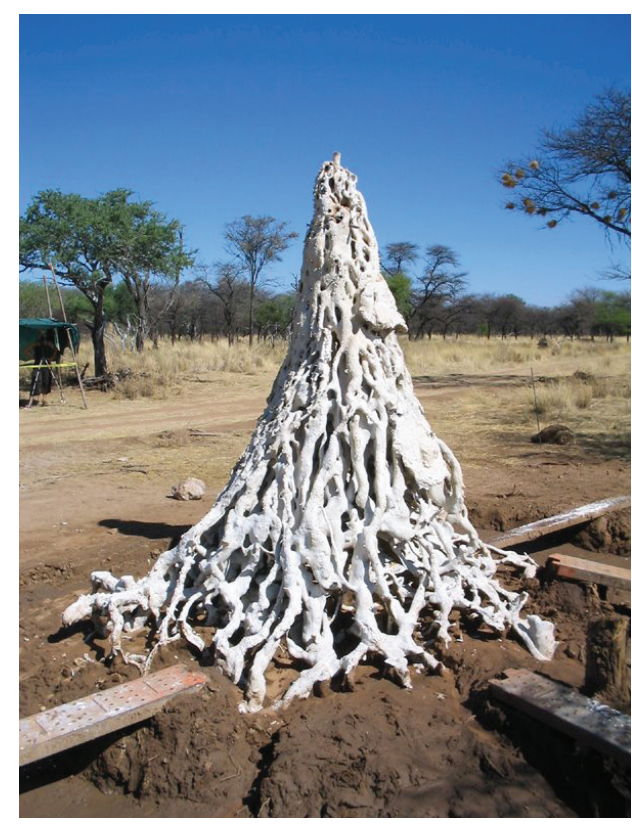

Figure $2 \mid$ To show the inside of a termite mound and to study "the perfectly smooth large tunnels that ran like veins right through them" researchers have cast termite mounds in plaster and subsequently washed away the soils. The photo shows the cast of a Macrotermes michaelseni mound. Everything that is white was empty space in the termite mound that could be filled with plaster (Haitham 2010). Photo credit: Turner (2011).

The termite-fungus symbiosis emerged at a single point in time and space, roughly 30 million years ago in the African rain forest (Aanen et al. 2002, Aanen and Eggleton 2005, Roberts et al. 2016). Because termites can create a climate that resembles the warm humid conditions of the rain forest, the cooperation with termites allowed Termitomyces to live and thrive in the more variable and unfavourable conditions of savannas (Aanen and Eggleton 2005). The fungus-growing termites and their fungi expanded their habitat throughout Africa and Asia (Figure 4). 


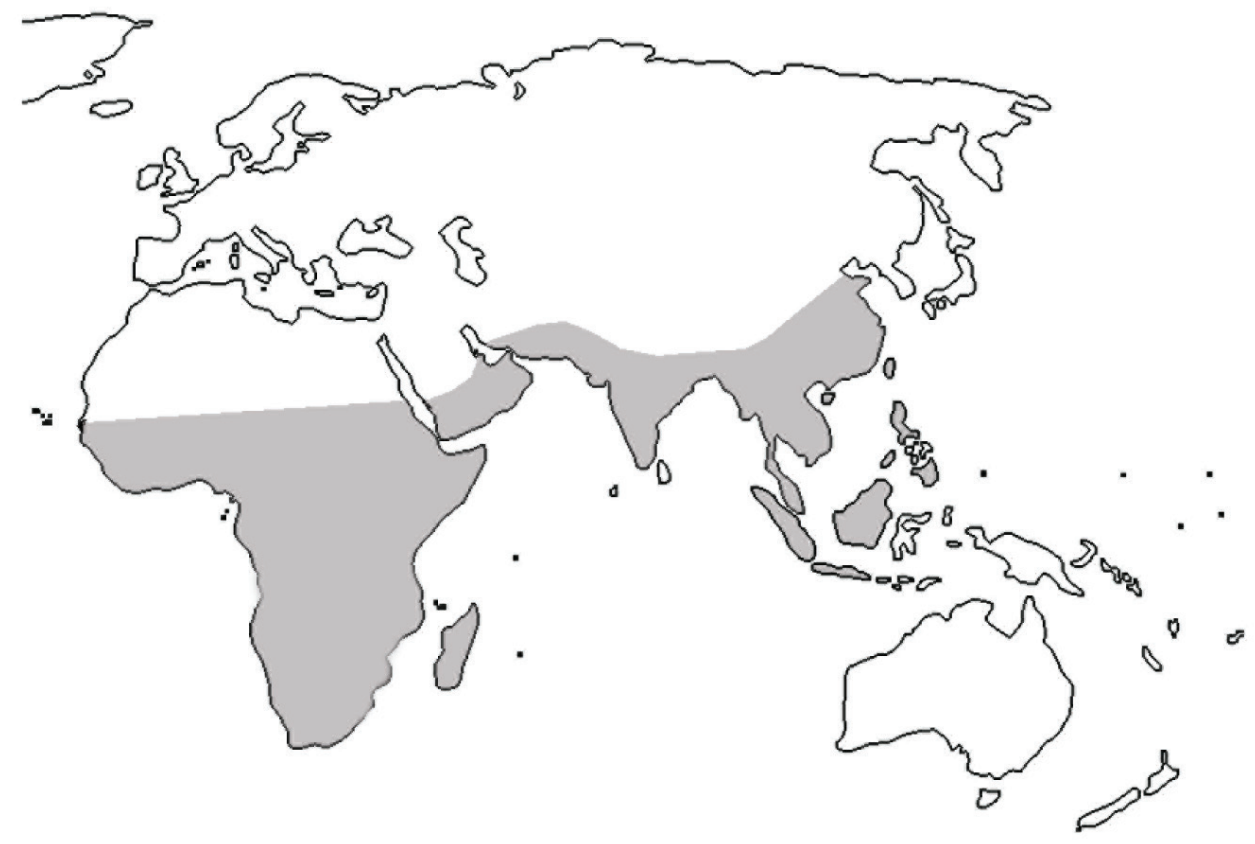

Figure 4| Fungus-growing termite distribution: grey area shows the approximate distribution of fungus-growing termites in the world. Figure was modified from Poulsen et al. (2014).

Consequently, between the origin of the symbiosis and now, as far as we know, 11 genera of fungus-growing termites have evolved (Eggleton 2000). At the moment, we know about 330 fungus-growing termite species and 40 Termitomyces species. All species of Termitomyces belong to the same genus (Aanen et al. 2002).

The success of fungus-growing termites is viewed somewhat ambiguously. On the one hand, fungus-growing termites are considered pest species that cause serious damage to crops and buildings (Mitchell 2002, Uys 2002). On the other hand, fungus-growing termites have almost reverently been referred to as 'ecosystem engineers' and key decomposers of arid areas; in savannas fungus-growing termites are responsible for up to $20 \%$ of all carbon mineralisation (Wood and Sands 1978). The termite mounds are fertility hotspots in nutrient poor areas and the foraging tunnels influence water dynamics in otherwise arid areas. Some even propose that termites, and especially fungus-growing termites, provide ecosystem services to arid and sub-arid regions in a way similar to the services that earthworms provide in temperate and humid tropical ecosystems (Jouquet et al. 2017). Whether perceived positively or not, the success of the fungus-growing termites can largely be attributed to their reliable symbiosis with Termitomyces. 


\section{Mutualistic symbiosis}

Symbiosis is a persistent co-existence between two or more different species; this co-existence has aided major transitions in evolution (Maynard Smith and Szathmáry 1995). For example, it is widely accepted that symbiosis resulted in the transition from prokaryotic cell to eukaryotic cell. Also, by bringing together species with a different pool of abilities, new combinations arise, and new ecological niches can be explored. Symbiotic relationships can range from being beneficial for both partners (mutualism) to being harmful for both partners (competition) (Figure 5). In general mutualistic symbioses beneficial to both partners - are thought to provide the best possibility for evolutionary novelty (Maynard Smith 1989). However, how mutualistic symbioses remain mutually beneficial and evolutionary stable has puzzled many evolutionary biologists.

Although the outcome of a mutualistic relationship is beneficial to both partners, this does not mean that there are no conflicts of interest between its partners. Mutualisms can even be viewed as reciprocal exploitations that provide a net benefit to both partners, despite the costs of the interaction (Herre et al. 1999). Moreover, natural selection should favour those mutualists that cheat: mutualists that increase their own fitness by

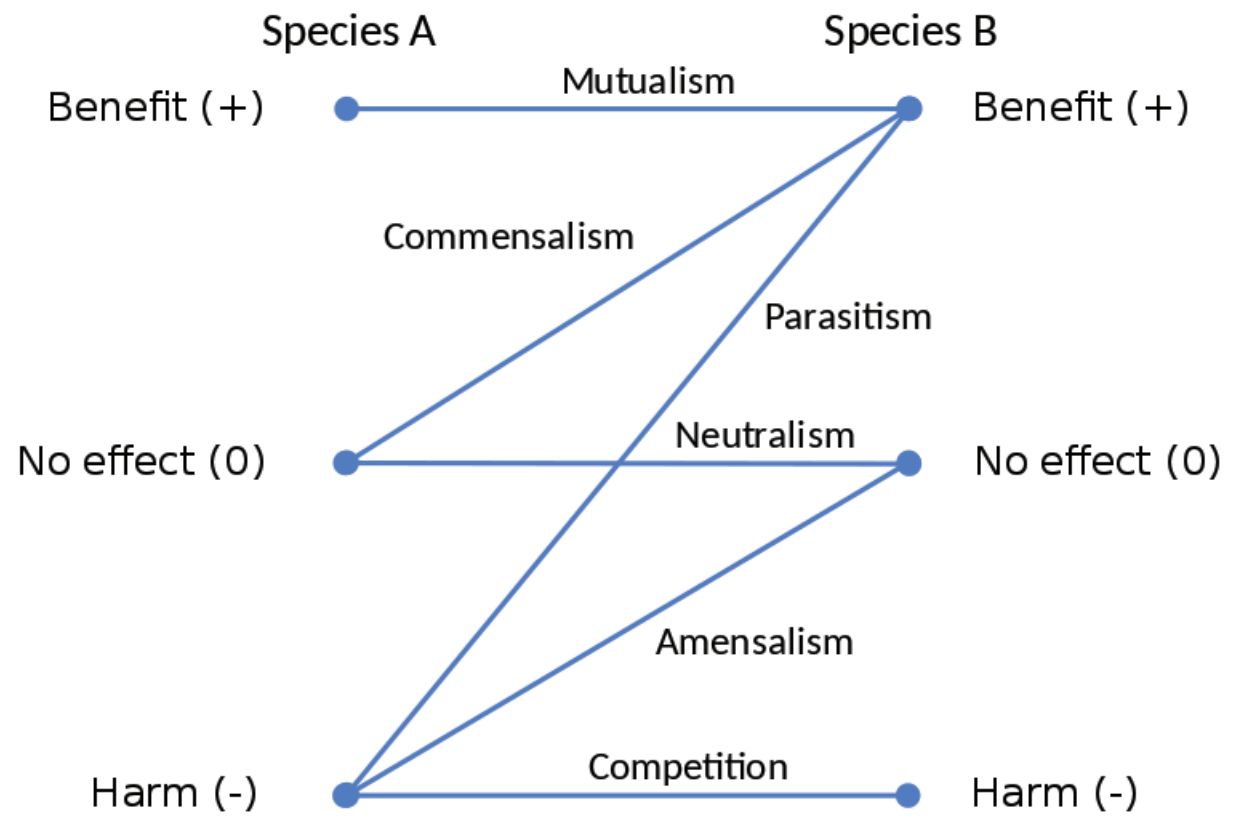

Figure 5 | A schematic representation of the possible types of symbiotic relationships and their effect on both partners. Figure by Alexander (2018). 
reducing the costs of 'benefitting' their partner. By cheating on one's partner the mutualism would shift from mutualism to commensalism, or even parasitism (Figure 5). Yet, there are many examples of ancient mutualistic relationships, such as that between termites and Termitomyces fungi. A major question in evolutionary biology is thus what stabilising mechanisms explain the myriad of mutualistic symbioses (Heath and Stinchcombe 2014).

\section{Stabilising mechanisms}

Mode of transmission, i.e. the way in which symbionts are transferred from one generation to the next greatly influences the degree of parasitism or mutualism (Maynard Smith 1989, Bull et al. 1991, Maynard Smith and Szathmáry 1995, Leigh Jr 2010). It must be noted that most hypotheses about stabilising mechanisms and theories about symbioses are about a larger host and a smaller endosymbiotic symbiont, as these are most abundant (Law and Lewis 1983, Frank 1996, Leigh Jr 2010). Therefore, I will now switch to the terms host and symbiont, rather than symbiotic partners. In the sections below, I will further extend on how these theories apply to the termite-fungus symbiosis.

Symbionts can be transmitted either horizontally, or vertically between generations. Vertical transmission means that a symbiont is passed directly from parent to offspring, whereas horizontal transmission means that a new relationship must be established with each new generation. In the case of vertical transmission, transmission can be uniparental or biparental, meaning that offspring inherits the symbiont from one or both parents respectively.

Vertical transmission reduces the possibility for cheating, because it aligns the reproductive interest of host and symbiont (Herre et al. 1999). Due to vertical transmission, the fitness of the symbiont becomes coupled to that of the host. Therefore, if the symbiont would reduce the fitness of its host, as a result it would also decrease its own fitness. A second stabilising result of vertical symbiont transmission is that, in general, it reduces the mixing of unrelated symbionts within the host. This is important for mutualism stability, because having multiple unrelated symbionts could lead to competition between the symbionts with possible detrimental side effects to the host (Frank 1996). Vertical transmission is usually uniparental, which results in even less symbiont diversity within a host.

Yet, in spite of the stabilising effect of vertical uniparental transmission, theory predicts that host and symbiont do not have the same interest when it comes to mode of transmission (Frank 1996). As explained above the host is under selection to reduce symbiont dispersal and the associated mixing between genetically different symbionts (Frank 1996). The symbiont, however, is under selection to disperse out of the host lin- 
eage to reduce competition with close relatives (Hamilton and May 1977, Frank 1996). Therefore, symbionts should always try to escape and favour at least some degree of horizontal transmission.

Next to mode of transmission there are other mutualism stabilising mechanisms. Two mechanisms that are often discussed in the context of horizontal transmission are partner fidelity and partner choice (Bull and Rice 1991, Herre et al. 1999, Sachs et al. 2004). Partner fidelity is defined by the degree of commitment between partners; the longer the interaction, the higher the commitment, the less incentives for cheating. The classic model for partner fidelity is the "Iterated Prisoner's Dilemma", which assumes individuals to be associated with the same partner for a number of interactions (Bull and Rice 1991). If an individual fails to cooperate in one interaction this is penalised by its partner in the next trial. So usually when we are talking about partner fidelity this also implies some sort of sanctioning mechanism, or a feedback mechanism to ensure cooperation over a longer period. In contrast, the partner choice mechanism is based on only one interaction (Bull and Rice 1991). One partner can distinguish how cooperative a partner is and change his reward accordingly (Bull and Rice 1991, Herre et al. 1999, Sachs et al. 2004). Partner choice thus always implies asymmetry between the interaction partners, i.e. one partner can choose whereas the other cannot, which is often the case in host symbiont interactions.

Finally, a result of a prolonged interaction between species can be coevolution, if the interaction has an impact on the reproductive success of the partners (Stearns and Hoekstra 2000). The extent of coevolution can have a stabilising effect on a mutualism; the more host and symbiont coevolve, the more specialised on each other they likely become. One way in which partners in a mutualism can become specialised on each other is if they become nutritionally dependent. Nutritional dependence can, for instance, mean that one species loses the ability to synthesise a nutrient that is reliably provided by their symbiont, and/or vice versa.

\section{Life cycle of the termite-fungus symbiosis}

Fungus-growing termites are an excellent example of a horizontally transmitted, yet ancient and stable mutualistic symbiosis. This means that each new termite colony needs to re-establish the interaction with a Termitomyces fungus. As I explained above, this begs the question how the interests of the termites and their fungi are aligned and how possible conflicts are mitigated. However, to be able to explore these questions, we first need to understand the life cycle of the termite-fungus symbiosis. I will start by explaining the life cycle between fungus-growing termite colonies, i.e. how a new termite colony is founded. Subsequently I will explain the within colony dynamics, i.e. how the Termito- 
myces symbiont is cultivated within a nest. Figure 6 is a wonderful schematic representation of how this sexual cycle (between colonies) and asexual cycle (within colonies) are coupled, made by Nobre et al. (2014).

Every single fungus-growing termite colony is founded by two winged, reproductive termites called alates. A male and female alate pair up and if the pairing is successful, they shed their wings and burry themselves in the soil (Wood and Sands 1978, Darlington 1994). These two alates become the king and queen of the mound (Figure 7). Together they produce all the other termites that form the society within the termite colony. Other castes (worker and soldier termites) are sterile; they are incapable of reproduction. The first workers that are produced by the new king and queen, leave the colony to forage for food, which they ingest and subsequently defecate to start the formation of the first fungus garden (also called fungus comb). At this point, the fungus garden needs to be inoculated with the Termitomyces symbiont, otherwise the colony dies (Johnson et al. 1981). If the inoculation is successful, the colony can further develop. In some species, it grows out into a mound that is a large, intricate structure with tunnels and chambers in which the conditions are tightly regulated (Korb 2003). Other species form below-ground colonies that also can reach large sizes, but do not form conspicuous above-ground structures (van de Peppel and Aanen 2020). Each colony houses thousands of termites and can persist for decades (Aanen et al. 2002).

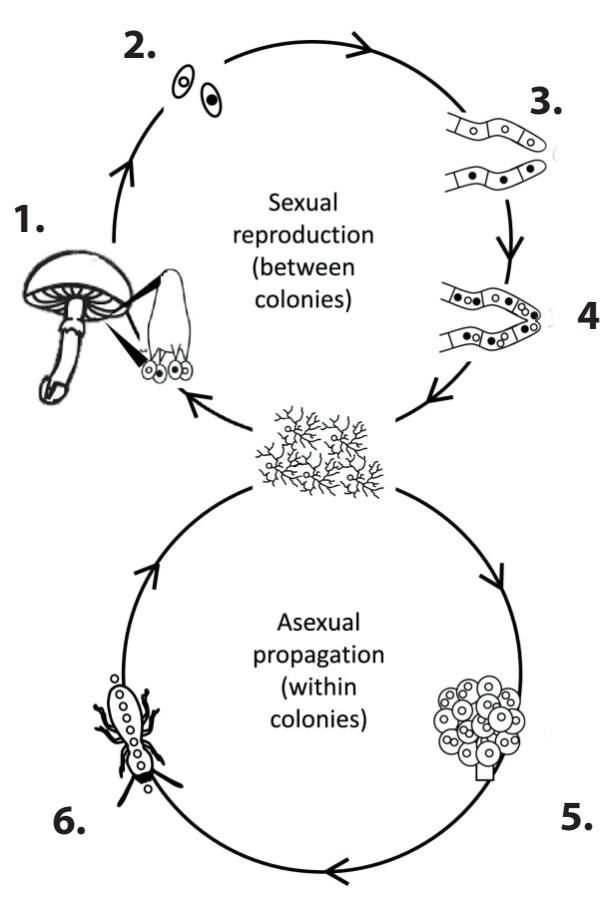

Figure 6| The within and between colonies life cylce of most Termitomyces species, modified from Nobre et al. (2014). The top circle shows the cycle between colonies. 1.) A Termitmyces fungus from an existing termite colony fruits; a mushroom appears on the termite mound. 2.) The mushroom makes sexual spores that spread through the environment. 3.) The sexual spores are picked up by workers from new termite colonies and germinate in the fungal combs to form homokaryotic mycelium: mycelium with one type of haploid nucleus. 4.) Homokaryons can fuse to form heterokaryotic mycelium: mycelium with two different types of haploid nuclei. The lower circle shows the asexual cycle within a termite colony. 5.) Fungal mycelium colonises the fungus comb and forms asexual spore balls called nodules. 6.) Termites consume the nodules and inoculate new parts of the fungus comb with the spores in their excrements (also see Figure 7). 


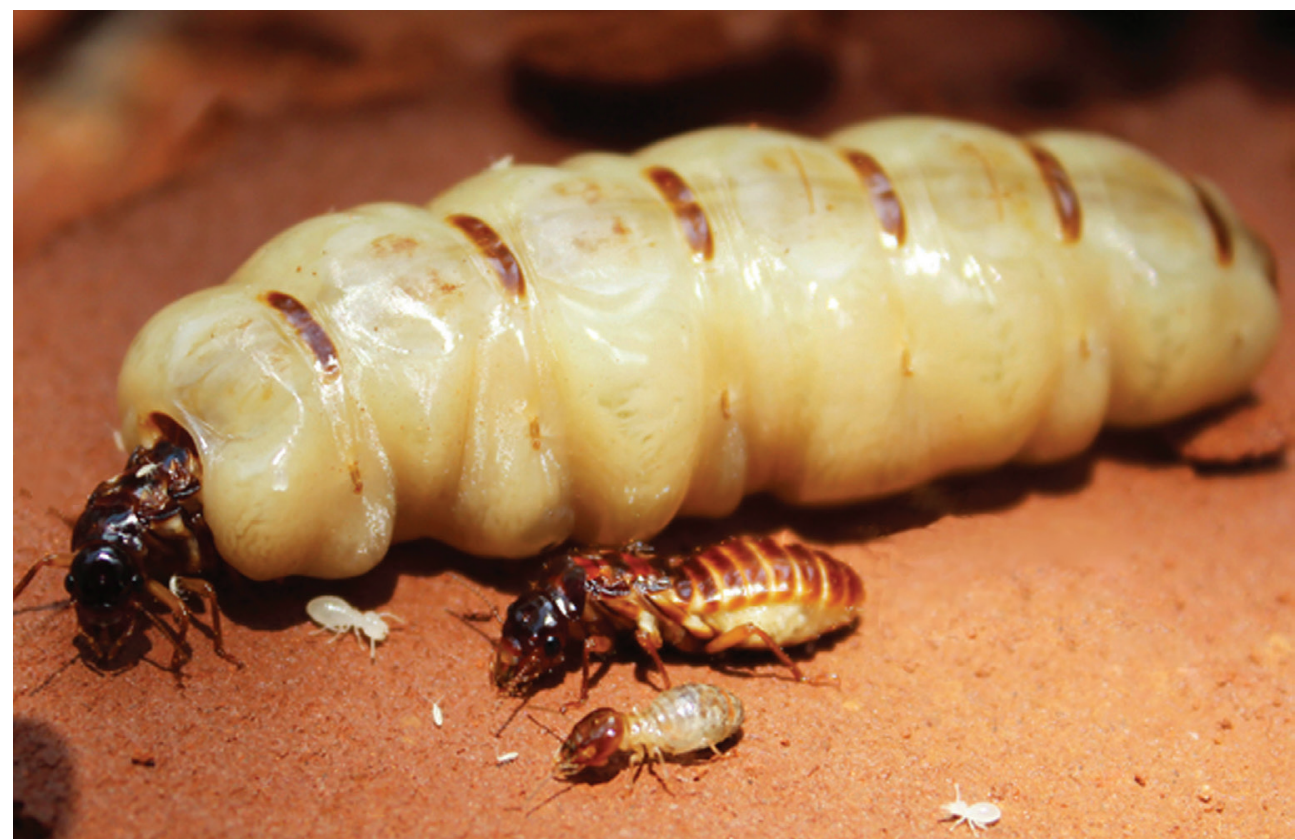

Figure 7| Termite queen (back), termite king (middle), termite worker (front), and nymphs (white). Photo credit: Saria Otani.

The termite colony mainly feeds on dead, although not much decomposed, plant material, e.g. leaf litter, dead grass, and dead wood, but also excrements of other animals such as elephant dung (Darlington 1994). The dead plant material is transported to the colony by termite workers, where it is consumed together with asexual Termitomyces spores and defecated to form the fungus comb: the first gut passage (Figure 8). The fungus comb is a cork-like substance that is essentially perfectly inoculated fungal substrate, allowing for rapid colonisation by the fungus. Once the comb is colonised, the fungus produces asexual spores in the form of white balls that grow on the fungus combs (Figure 9). These asexual spore balls have been described by all researchers that studied fungus-growing termites and are thought to be present in all Termitomyces species (e.g. Petch 1906, Heim 1942, Batra and Batra 1967, Leuthold et al. 1989, Darlington 1994). The spore balls are most often referred to as nodules, however, they have also frequently been called mycotêtes (Heim 1942, Wood and Sands 1978) or spherules (Batra and Batra 1966). The nodules, of larger species especially, look and feel somewhat like tiny Styrofoam balls. Finally, the oldest fragments of the comb, of which the nodules have already been harvested, are eaten again by termite workers: the second gut passage (Figure 8). 


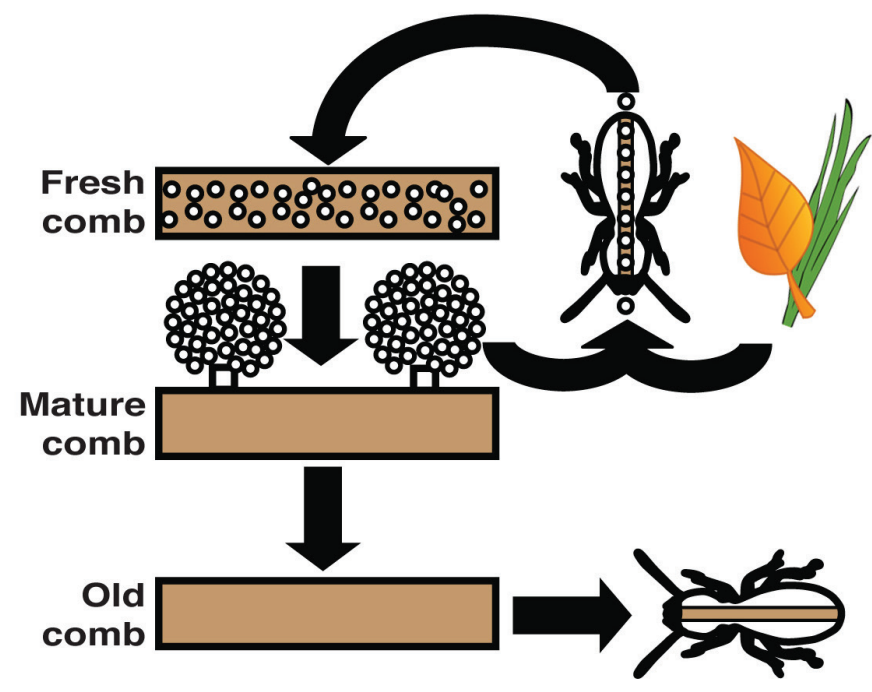

Figure 8| A schematic representation of how Termitomyces is grown and propagated within the termite colony, modified from Aanen and Boomsma (2006). Dead plant material is collected and brought to the colony where it is consumed by young workers together with asexual Termitomyces spores from nodules. After a short gut passage this inoculated substrate is defecated to form new fungus comb. The fungus colonises the substrate and forms new nodules. Old substrate is consumed by old workers for a second gut passage.

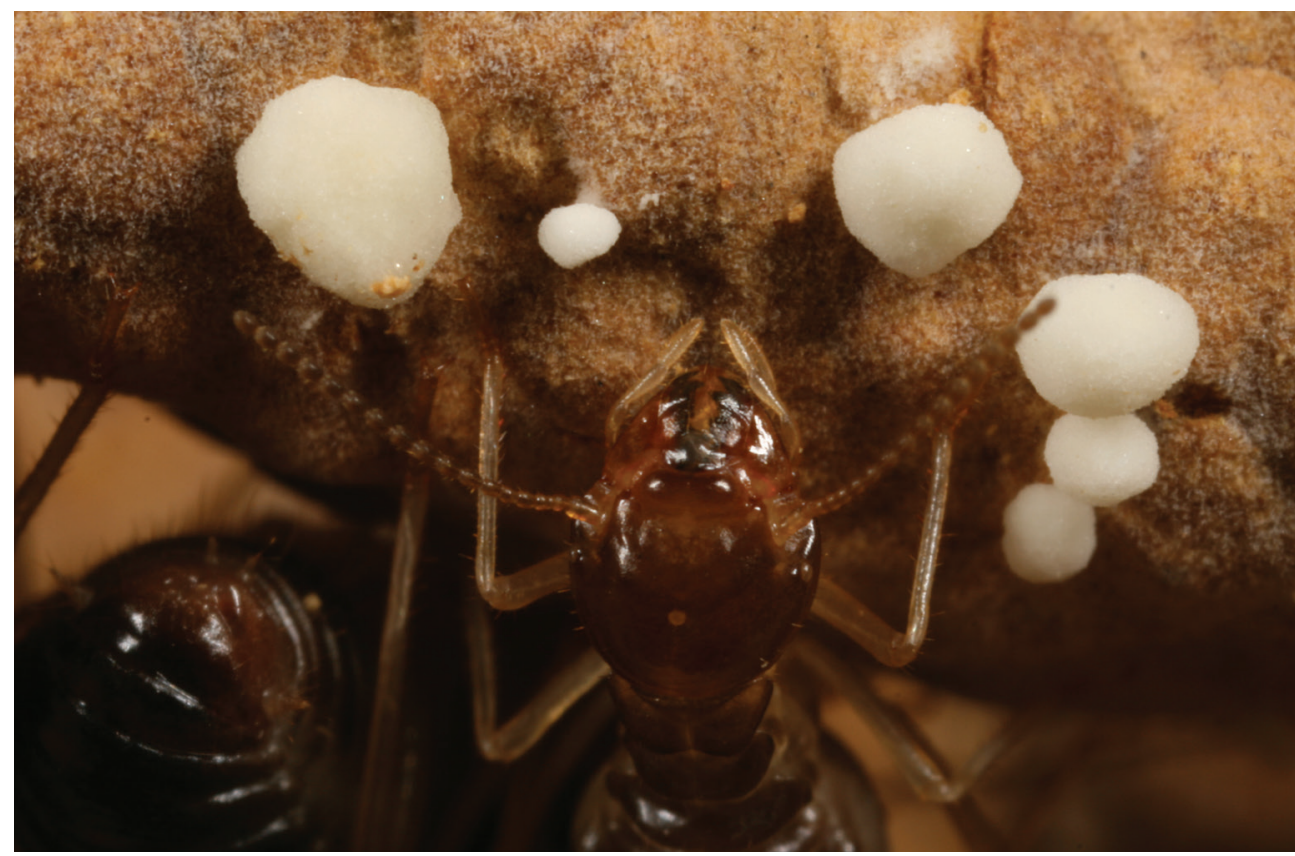

Figure 9| Macrotermes natalensis termite worker on a fungal comb with nodules. Photo credit: Koos Boomsma. 
The within-colony cycle of fungus-growing termites has best been described for species of two termite genera with larger termites: Macrotermes and Odontotermes (Badertscher et al. 1983, Gerber et al. 1988, Leuthold et al. 1989, Li et al. 2016). All associated studies show the same general principles for an age-related division of labour between young and older workers in the cycling of food through the colony. Young workers maintain the fungus comb. They consume nodules and plant material and are thus responsible for inoculating the fungus comb with asexual fungal spores from nodules. Older workers are responsible for foraging and mainly consume old fungus comb to produce the final faeces. In contrast to this age-polyethism that was found for Macrotermes and Odontotermes species, studies of some Microtermes species and Ancistrotermes species did not find indications for this division of labour (Aanen and Boomsma 2006).

\section{Symbiont transmission in the termite-fungus symbiosis}

As explained in the section above, once the fungus comb is initiated by the first workers of a new termite colony, it needs to be inoculated with its fungal symbiont. In most fungus-growing termites, the Termitomyces symbiont is obtained from the environment through horizontal transmission (Sands 1960, Johnson et al. 1981, Sieber 1983, Korb and Aanen 2003, De Fine Licht et al. 2006). Only two independent evolutionary transitions to vertical transmission have likely occurred: in one species of Macrotermes and in all known species of Microtermes (Johnson 1981, Johnson et al. 1981, Nobre et al. 2010). In fungus-growing termite species with vertical transmission, transmission is indeed uniparental. Only one of the two alates, either the male alate (in Macrotermes bellicosus), or the female alate (in all studied species of Microtermes) brings asexual spores from the mother colony to inoculate its newly founded colony Johnson 1981, Johnson et al. 1981, Nobre et al. 2010).

For horizontal transmission of the Termitomyces symbionts, it is necessary that the fungus can be found in the environment. As Termitomyces fungi are not found in a free-living state, it is unlikely that the fungus can be found as mycelium, the vegetative growth form of a fungus. Also, Leuthold et al. (1989) found that mycelium does not survive gut passage, thus making it even more unlikely that fungal mycelium is the source of horizontal symbiont transmission. Finally, asexual spores from the Termitomyces nodules are unlikely to be spread in the environment as they remain inside the colony and are not wind dispersed. By deduction, horizontal symbiont transmission is likely achieved through obtaining wind-dispersed sexual spores, which are spread by the fungal fruits: mushrooms. This is in line with a study of the population structure of the fungal symbiont of Macrotermes natalensis, in which a signature of free recombination was found (De Fine Licht et al. 2006). Free recombination indicates that there is a sexual stage, 
i.e. sexual spore formation in mushrooms, between symbiont transmission from one colony to the next.

For sexual spores to be spread in the environment, the mushrooms that produce these spores need to appear. For this to happen, existing mounds need to allow their fungus to produce mushrooms. This is where there is room for conflict between the termite host and the fungal symbiont. It is the embodiment of the conflict over symbiont transmission mode, which I described in the section "stabilising mechanisms". Firstly, a conflict over resources allocated to sexual reproduction emerges. The mushrooms of some Termitomyces species are reported to weigh about $2.5 \mathrm{~kg}$ (Piearce 1987), thus consuming a substantial amount of mound resources. Yet, there is no direct fitness benefit to the existing mound to allow its fungus to fruit (Aanen and Boomsma 2006). Vice versa, when alates disperse, an estimated $40 \%$ of the colony biomass disappears (Wood and Sands 1978), leaving fewer termites to provide the fungus with substrate. Secondly, there is the conflict over symbiont mixing: each new termite colony is prone to being inoculated with multiple, unrelated Termitomyces fungi. As each new termite colony must obtain its Termitomyces symbiont via inoculation with two compatible sexual spores (two homokaryons that later form a heterokaryon, see Figure 6), this implies that sexual spores are abundant in the environment. Therefore, it is likely that starting termite colonies are indeed inoculated with multiple Termitomyces fungi, possibly introducing competition between fungal individuals.

\section{Endosymbiosis}

Before I continue on the stabilising mechanisms we know of in the termite-fungus symbiosis, we need to address the question whether Termitomyces is an endosymbiont - a symbiont that lives within a host - or not (ectosymbiont). Long-term mutualisms - mutualisms in which at least one partner species spends over half of its life associated with the other (Douglas 2010, Leigh Jr 2010) - often involve a larger host and a smaller endosymbiotic symbiont that belong to different kingdoms (Law and Lewis 1983, Frank 1996, Leigh Jr 2010). The latter tends to bring together species with complementary abilities, which benefits both species. The host is often the dominant partner that has some form of control over its symbionts to prevent them from becoming parasites (Douglas 2010, Leigh Jr 2010). Most theories about mutualism stabilising mechanisms that I explained above are about larger hosts and endosymbiotic symbionts.

One could argue that for a single termite, Termitomyces is not an endosymbiont. However, it has often been suggested that a termite colony can and should be viewed as one superorganism. As Eugène Marais puts it: "You must consider a termitary as a single animal, whose organs have not yet been fused together as in a human being" (Marais 
1937). The reason that a colony of fungus-growing termites can indeed be viewed as one organism is because fungus-growing termites are highly eusocial insects (Wheeler 1911). Boomsma and Gawne (2018) have reviewed the term "superorganism" from this perspective as I will extend on below. A similar case concerning endosymbiosis has been made for the fungus-growing ants and their symbionts (Poulsen and Boomsma 2005, Kooij 2013).

By definition, insects have to fulfil three criteria to be considered eusocial (Wilson 1971). The first criterion is cooperative brood care, which means that individuals take care of offspring that is not directly their own. The second criterion is that there is reproductive division of labour, which means that some individuals forgo reproduction and only take care of the offspring of other individuals from their colony. The third criterion is that is overlap of adult generations, which is thought to increase the amount of parental care the offspring receive (Queller 1994). In fungus-growing termites, the eusociality is so extreme that only two individuals within a colony reproduce (the king and queen) and these two individuals are mated for life. This means that the population of the termite colony can be compared to the germline (sex cells) and soma (all other cells) in a multicellular organism. There are a few cells that can reproduce (the germline) and all the other cells support these germline cells, forgoing reproduction. From the superorganism perspective, the fungus-growing termite colony can indeed be considered a superorganism, which would make their Termitomyces fungi endosymbionts rather than ectosymbionts.

An additional argument for considering a host-endosymbiont relation between the termites and their fungi, follows from the fact that many more fungus-growing termite species have been found than Termitomyces species (Eggleton 2000). Law and Lewis (1983) found that inhabitant symbionts are represented by much smaller taxonomic diversity than exhabitant hosts, which is in line with what we observe in the termite-fungus symbiosis. However, it must be noted that it is likely that the currently known Termitomyces species are an underrepresentation of the actual number of Termitomyces species, because the Termitomyces taxonomy is largely based on the morphology of mushrooms. The mushrooms are rare and possibly absent in some Termitomyces species (Nobre et al. 2011c) and some Termitomyces species may not be distinguishable by mushroom morphology (FrØSlev et al. 2003).

In this thesis, I consider Termitomyces to be an endosymbiont, which means that we can investigate whether the theories on mechanisms that stabilise mutualisms apply to the fungus-growing termites and their symbiont. Being in a host-symbiont relationship implies that the termites are likely to have a more dominant function controlling parasitic tendencies of their Termitomyces fungi. 


\section{Known stabilising mechanism in the termite-fungus symbiosis}

One of the mechanisms that stabilise the termite-fungus symbiosis, was elucidated by Aanen et al. (2009) and later expanded on by Nobre et al. (2014). Each fungus-growing termite colony only houses one individual heterokaryotic fungus; the fungus has two different haploid nuclei, comparable to a diploid organism (Figure 6). However, the colonies are inoculated with homokaryotic spores - with only one haploid nuclear type (De Fine Licht et al. 2005). This means that at the start of each colony there must be multiple -at least two, likely more- inoculations with homokaryotic spores. Aanen et al. (2009) found the resulting heterogeneity is mitigated and resolved by positive frequency dependent selection of the fungus: because the termites inoculate each piece of fungus comb with asexual spores from nodules, that fungus that makes most nodules will be present most in new parts of the fungus comb, leading to more nodules et cetera. Also, Aanen et al. found that strains in a mixture with a higher frequency produced disproportionally more of the resulting spores (so more than based on their initial frequency) than strains with a lower frequency. The observed increase in spore production of the most frequent heterokaryon is likely the result of fusion between germinating spores of the most frequent strain (Aanen et al. 2009). Furthermore, as heterokaryons, resulting from a mating between two compatible homokaryons, make more spores than homokaryons (Nobre et al. 2014), this mechanism ensures that as the mound matures only one, heterokaryotic fungal symbiont remains.

We now know how a single heterokaryotic fungal symbiont remains in a mature termite colony. We do not know, however, how the 'correct' symbiont is selected. As explained above, the more host and symbiont depend on each other, the more coevolution should occur. In host-symbiont interactions with strict vertical transmission there should be a high degree of coevolution and a high degree of mutual specificity (also termed interaction specificity; Aanen et al. 2007). In the termite-fungus symbiosis, however, transmission mode does not seem to reflect the interaction specificity that is observed between termites and their fungi (Aanen et al. 2007, Nobre and Aanen 2012, van de Peppel and Aanen 2020). There are species with vertical transmission that have fungal symbionts that are not monophyletic (Aanen et al. 2002, Nobre et al. 2011a, Nobre et al. 2011b, van de Peppel and Aanen 2020), and vice versa there are species with horizontal transmission that are specialised on one fungal symbiont (De Fine Licht et al. 2006, Aanen et al. 2007). The latter especially implies that a mechanism exists that ensures a high interaction specificity despite horizontal symbiont transmission. 


\section{Outline of this thesis - stabilising mechanisms and possible room for conflict}

The mechanism that minimises symbiont competition within the host is a large part of the puzzle that explains the stability of the termite-fungus symbiosis. However, we do not know how the conflict over resource allocation associated with independent sexual reproduction and dispersal is resolved. Nor do we know the mechanism that allows the host to 'choose' the symbiont that fits best. The aim of this thesis is to explore possible mechanisms for stabilisation of the fungus-termite mutualism as well as to explore other possibilities for conflict within symbiosis.

It has been hypothesised that the conflict over resource allocation is mitigated by alignment of the moment at which host and symbiont reproduce. The idea is that when the alates leave the colony - this happens synchronously - less fungus is consumed and the fungus can escape the termite's suppression of mushroom formation (Aanen and Boomsma 2006). This fits data from Kone et al. (2011), who have shown that Termitomyces mushrooms are only found on termite mounds that have produced alates. Also it is in line with the observation that Termitomyces mushrooms appear shortly after the alates have dispersed from their mother colonies (Johnson et al. 1981, Darlington 1994). A crucial component of the hypothesised mechanism is that the asexual nodules are also the primordia - the beginnings - of mushrooms. When they are no longer consumed by termites they can grow out into the precious Termitomyces mushrooms. In chapter 2 we address a part of this hypothesis by testing whether nodules of the symbiont of Macrotermes natalensis indeed have the capacity to grow into mushrooms when they are not consumed by termites.

Positive frequency dependent symbiont selection explains mechanistically how one single Termitomyces heterokaryon is selected by the termites. Yet, it does not explain the interaction specificity that is observed within the termite-fungus symbioses. To explain the observed interaction specificity and co-cladogenesis amongst the termites and their fungal symbionts several studies have suggested that the substrate that is provided by the termites plays a role in the selection of the fungal symbiont (Rouland-Lefèvre and Bignell 2002, Nobre and Aanen 2012, Kone et al. 2013). If the type of substrate suits the Termitomyces individual, the fungus will be able to produce more asexual spores. The fungus that produces most asexual spores will be selected. In chapter 3 we explore whether Termitomyces isolates from two different genera of termites have different growth capacities on a range of substrates. A difference in growth capacity could explain why the fungus of one termite species we studied is never found with termites of two species from the other genus we studied. We test growth capacity by performing single-factor growth assays - where we vary only the type of carbon source -, and by 
a two-factor nutritional geometric framework experiment - where we simultaneously vary carbohydrate and protein ratios.

In chapter 4 we make a more contiguous and complete version of the reference genome assembly of the Termitomyces symbiont of $M$. natalensis and present the first genetic linkage map of a Termitomyces fungus with two aims in mind. On the one hand we provide a tool with which further studies of Termitomyces will be facilitated. A more contiguous assembly will for example help QTL studies, comparative genomics between different Termitomyces isolates, and analysis of metabolic capacities. On the other hand, we use the genetic linkage analysis to study the recombination landscape of a full-sibling mapping population of this Termitomyces species. In the context of mutualisms this is especially interesting, because Law and Lewis (1983) and Bergstrom and Lachmann (2003) both show that selection for genetic change in endosymbionts is low compared to free-living relatives because of the mutualistic interaction.

In chapter $\mathbf{5}$ we zoom in on the peculiarities of the basidiomycete life cycle. The basidiomycete life cycle differs from that of many other organisms in ways that can lead to genomic conflict. In many organisms, when gametes fuse, the nuclei of the gametes fuse too to form a diploid, which links the faiths of the individual gametes. In basidiomycete fungi, two monokaryons - mycelia with one type of haploid nucleus - fuse to form a dikaryon - mycelium with two compatible haploid nuclei. In contrast to most organisms, however, the nuclei of the dikaryon remain separate, which leaves room for competition between nuclei. In addition, in contrast to the fusion of two gamete cells (e.g. an egg and sperm), in basidiomycetes two multicellular homokaryons fuse. Both homokaryons act as the donor of nuclei and as receptor of nuclei, taking on the male and female role simultaneously. In this chapter we explore the possibilities of genomic conflict in the context of the text-book basidiomycete life cycle. Also, I will extend the discussion in chapter 5 to the life cycle of Termitomyces that does not follow the textbook basidiomycete life cycle in all aspects.

In chapter 6 I discuss the findings of this thesis in the light of mutualism stability. I will elaborate on the origin of nodules in the termite-fungus symbiosis and elaborate on an alternative hypothesis to explain co-occurrence of the termite alates and fungal mushrooms.

\section{Final introductory remarks}

For centuries, probably even longer but without documentation, people have marvelled at the immensely complicated structures built by termites (Figure 10). I was lucky to spend my whole $\mathrm{PhD}$ on this intriguing symbiosis. I got to travel to South Africa, around the area of Pretoria and walk in the footsteps of perhaps the most famous ter- 
mite researcher so far: Eugène Nielen Marais writer of Die Siel van die Mier (The Soul of the White Ant). I am forever grateful to the people of FABI for sharing their labs, their knowledge, their fun and their childhood memories (see the first paragraphs of this introduction). In this thesis I hope to take you along on this wonderous journey into the termite-fungus mutualism.

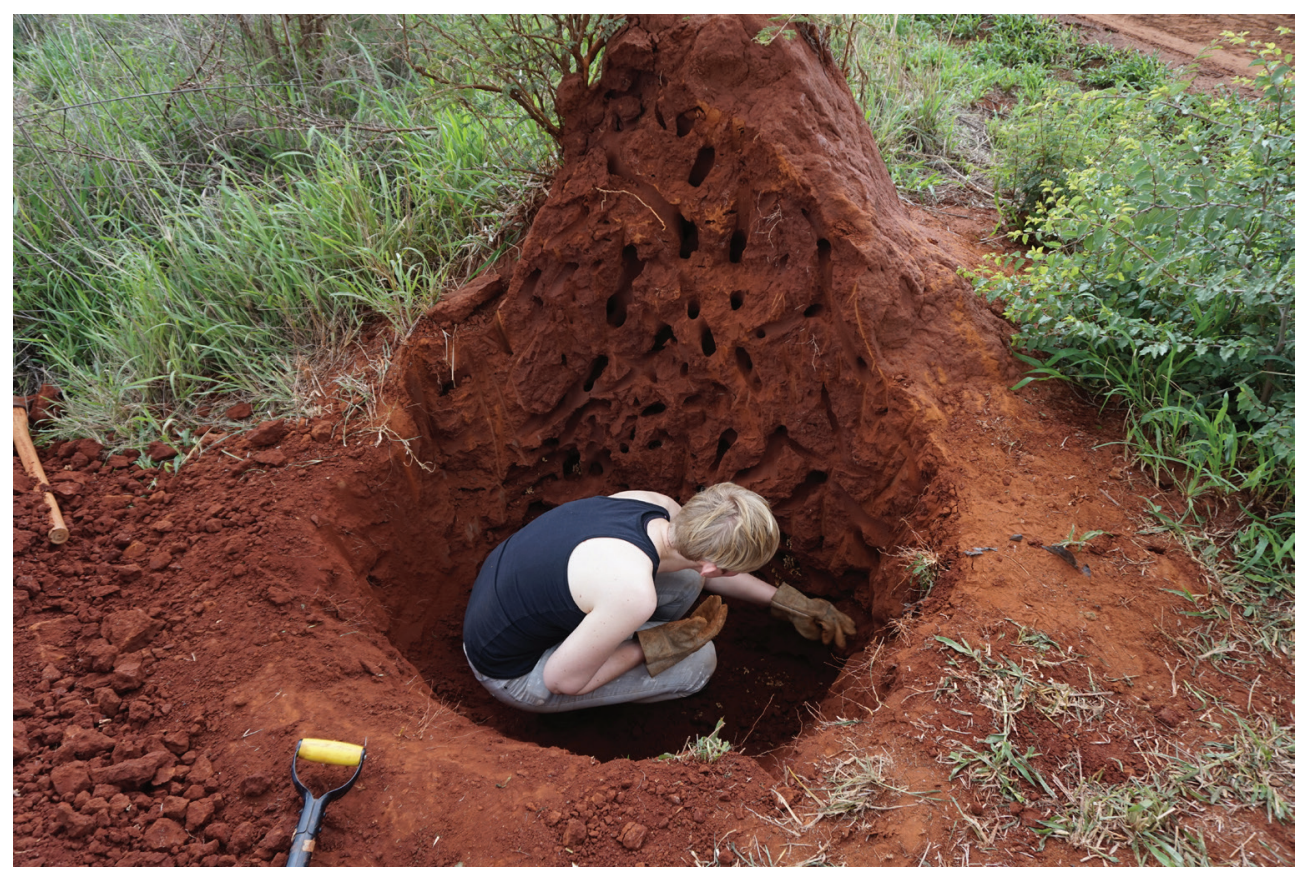

Figure 10 A M. natalensis termite mound, opened to examine the fungus garden. The above ground mound shows the intricate shafts that are built by the termites. Above ground is, however, not where most termites are found. The fungal combs and royal chamber are found belowground. In this photo I am carefully taking pieces of fungus comb from the colony. I am wearing gloves because the termite soldiers bite to protect their colony. This mound was pivotal to the main finding of this thesis. It was the first mound in which we discovered pointy nodules, the true mushroom primordia (see chapter 2). 


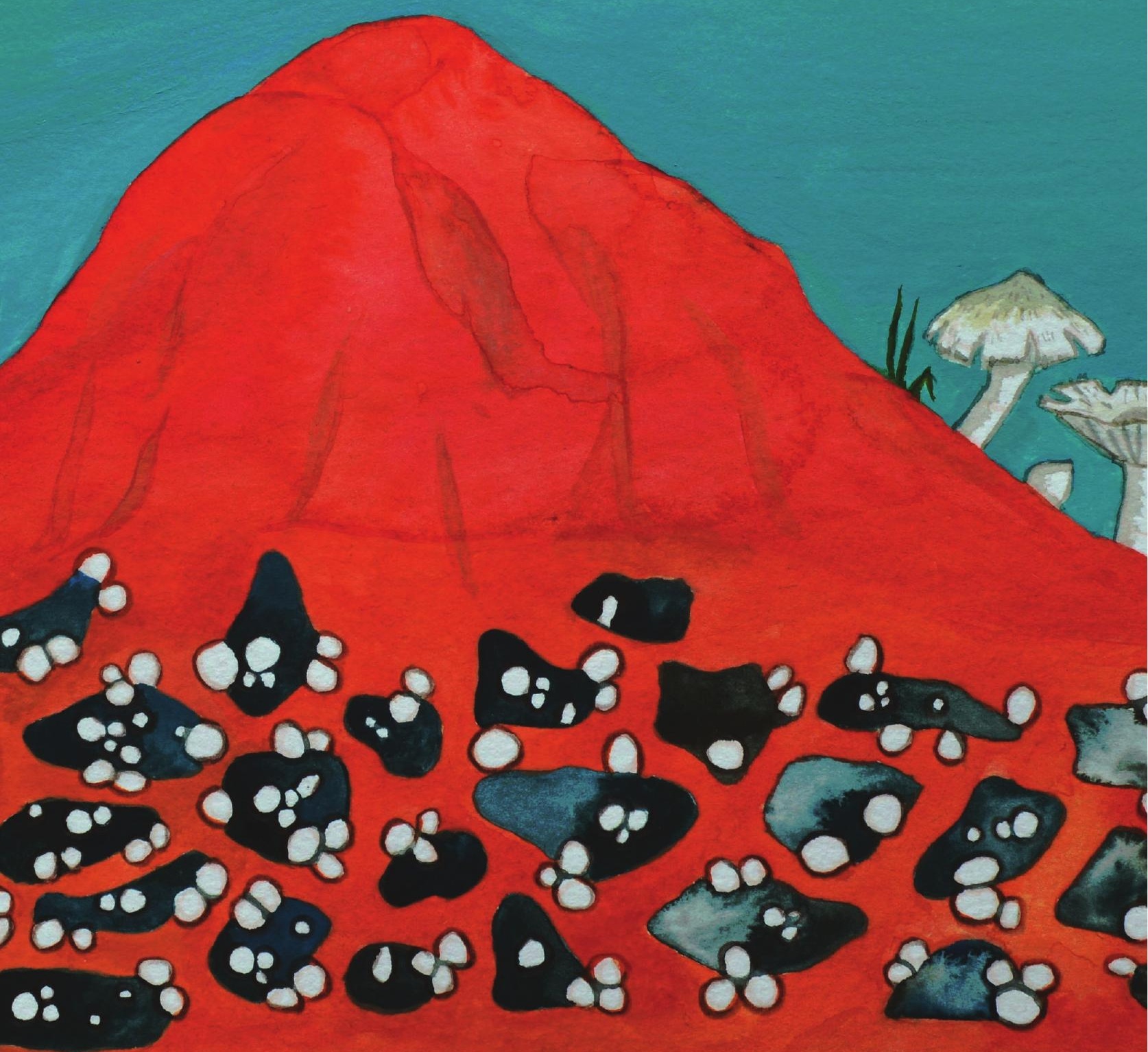




\section{Abstract}

Although mutualistic symbioses per definition are beneficial for interacting species, conflict may arise if partners reproduce independently. We address how this reproductive conflict is regulated in the obligate mutualistic symbiosis between fungus-growing termites and Termitomyces fungi. Even though the termites and their fungal symbiont disperse independently to establish new colonies, dispersal is correlated in time. The fungal symbiont typically forms mushrooms a few weeks after the colony has produced dispersing alates. It is thought that this timing is due to a trade-off between alate and worker production; alate production reduces resources available for worker production. As workers consume the fungus, reduced numbers of workers will allow mushrooms to 'escape' from the host colony. Here we test a specific version of this hypothesis: the typical asexual structures found in all species of Termitomyces - nodules - are immature stages of mushrooms that are normally harvested by the termites at a primordial stage. We refute this hypothesis by showing that nodules and mushroom primordia are macro- and microscopically different structures and by showing that in the absence of workers, primordia do, and nodules do not grow out into mushrooms. It remains to be tested whether termite control of primordia formation or of primordia outgrowth mitigates the reproductive conflict. 


\section{Introduction}

All known species of the basidiomycete genus Termitomyces grow in a remarkable, obligate symbiosis with termites of the subfamily Macrotermitinae (Aanen et al. 2002). This farming symbiosis, in which termite hosts grow fungal symbionts for food in exchange for substrate and shelter, has attracted the interest of many ecologists and evolutionary biologists (e.g. Petch 1906, Batra and Batra 1967, Wood and Sands 1978, Darlington 1994, Aanen 2006). A major conundrum in the termite-fungus symbiosis is how the reproductive interests of host and symbiont are aligned, despite their independent dispersal in most fungus-growing termite species (Aanen et al. 2002, Aanen et al. 2007).

Termitomyces fungi have both an asexual and a sexual life cycle (Darlington 1994). The asexual cycle is the dominant lifecycle in a colony, while the sexual life cycle is required for symbiont dispersal to new colonies (Korb and Aanen 2003). Within a colony the fungus is grown on airy structures of plant substrate, called the fungus comb. The fungus colonises the comb and subsequently forms spherical structures that contain asexual spores: nodules. These nodules are consumed by termites together with plant material and defecated to form new fungus comb, thereby completing the asexual cycle (Leuthold et al. 1989). For sexual reproduction, the fungus forms sexual fruiting bodies: mushrooms (Heim 1977). These mushrooms have their origin in the fungus comb and pierce their way up to the surface of the termite mound. Once matured, they spread sexual spores throughout the environment, which are picked up by foraging termites to inoculate newly founded, fungus-less termite colonies (Johnson et al. 1981, Darlington 1994, Nobre et al. 2011a).

Paradoxically, while most fungus-growing termite species are dependent on acquiring their symbiont from spores in the environment (Sieber 1983, Aanen et al. 2002), it is not in the short-term interest of any individual termite colony to allow its fungus to fruit (Korb and Aanen 2003). Production of fruiting bodies wastes resources that could otherwise have been allocated to growth of the colony and ultimately to more alates. This has led multiple researchers to argue that the termites actively suppress fruiting body formation of their fungal symbiont (Batra and Batra 1967, Korb and Aanen 2003, Aanen 2006, Aanen and Boomsma 2006). Indeed it seems plausible that fewer workers can be produced to maintain the fungus comb, when alates are produced by a colony, and fewer mushroom initials will be eaten (Wood and Sands 1978, Aanen 2006, Aanen and Boomsma 2006, Kone et al. 2011). As a more specific corollary of this idea, it has been speculated that, in response to consumption of mushrooms at a primordial stage, the fungus would have evolved gut-resistant asexual spores on the unripe mushrooms, leading to the typical asexual structures found in all species of Termitomyces: nodules (Heim 1977, Leuthold et al. 1989, Aanen 2006, Aanen and Boomsma 2006). According 
to this hypothesis, these ubiquitous nodules are the initials of mushrooms that can develop into sexual fruiting bodies if not eaten by termites (Bathellier 1927, Sieber 1983, De Fine Licht et al. 2005).

Here we set out to test the latter assumption. Under the assumption that nodules are unripe mushrooms, nodules on fungus-comb fragments incubated in the absence of termites should develop into mushrooms. Also, since the inner structure of initials of other basidiomycetes shows clear mushroom features at very early stages (Bonner et al. 1956, Moore 1994), we hypothesised that if the nodules were equivalent to these stages of mushroom formation, they should show similar differentiation into mushroom.

\section{Materials and Methods}

\section{Excavations and fungus comb incubations}

A minimum of 15 fungus comb samples were excavated from 25 mature Macrotermes natalensis colonies in January and February 2015, 2016 and 2018. We chose to study the combs of this particular termite species, because it has been found that all Termitomyces strains associated with M. natalensis belong to the same biological species (De Fine Licht et al. 2005, De Fine Licht et al. 2006, Aanen et al. 2007, Nobre et al. 2014) and because the shape of its nodules can be studied with the naked eye. Fungus combs were carefully transferred to plastic zip-lock bags. The zip-lock bags were transferred to the laboratory in a plastic container and kept overnight at $4^{\circ} \mathrm{C}$.

The next day, wet, sterilised chromatography or filter paper was placed inside a sterile Microbox container (model O118/50+OD118, white filter) and two mL of sterilised, demineralised water was added to each container to maintain high humidity. A subset of 110 fungus combs from 12 colonies were transferred to each Microbox and any remaining termites were removed using sterilised forceps. The chambers were incubated in the dark at $25^{\circ} \mathrm{C}$. The fungus combs were regularly inspected for mushroom formation (Supplementary Table 1). In line with previous observations, as many as 29 combs were overgrown with other fungi, mainly Psendoxylaria, within four days of incubation (Supplementary Table 1; Thomas 1987b, Visser et al. 2009, Visser et al. 2011). These 29 fungus combs were removed.

\section{Basidiospore germination}

To check basidiospore viability, spore prints were made from three mushrooms of different combs on agar plates. The cap of the mushroom was cut off and attached with Vaseline to the lid of a Petri dish with malt yeast extract agar (MYA) medium (20g malt, $2 \mathrm{~g}$ yeast extract, $15 \mathrm{~g}$ agar in $1 \mathrm{~L}$ of demineralised water) for time periods ranging from 10 seconds to one hour. After incubation at $\sim 25^{\circ} \mathrm{C}$ germinating spores were individually 
transferred to a fresh Petri dish with MYA medium. All mushrooms produced viable homokaryotic spores, which was confirmed by mating experiments.

\section{Fixation and embedding of nodules}

Normal nodules and primordia were carefully taken off from a fungus comb using a small brush. Thin slices of opposite vertical sides of the nodules were cut off to increase fixation speed and accessibility during infiltrations and allow positioning of the nodules in embedding moulds. Nodules were put in at least five times their volume of fixative (4\% paraformaldehyde, $0.1 \%$ glutaraldehyde, and $0.05 \%$ Triton P40 in $0.05 \mathrm{M}$ PBS pH 6.8) and submerged by creating a low pressure until they sunk. Samples were kept at $4^{\circ} \mathrm{C}$ until embedding.

Fixed samples were dehydrated for at least ten minutes in 10\%, 30\%, 50\%, 70\%, $90 \%$ and two times in 100\% ethanol followed by gradual resin infiltration (Technovit 7100 (T7100); resin A: $100 \mathrm{ml} \mathrm{T7100,} 1$ bag of hardener I and 2,5 ml PEG 400). Samples were gently rotated for a minimum of $1 \mathrm{hr}$ at $30 \mathrm{rpm}$ with resin A:ethanol mixtures (resp. 1:3, 1:1 and 3:1), followed by o/n rotation in 100\% T7100 infiltration solution (A). Bottoms of the moulds were covered with a small layer of T7100 polymerisation solution (resin B: $15 \mathrm{ml}$ infiltration solution A and $1 \mathrm{ml}$ hardener II). Samples were quickly transferred, oriented and covered with polymerisation solution. Moulds were covered with a sheet of plastic, kept at RT for one hour, followed by $37^{\circ} \mathrm{C}$ incubation for one hour. Hardened embedded blocks were attached to microtome sample holders with freshly made Technovit 3040 glue. Longitudinal midplane sections $(4 \mu \mathrm{m})$ were made, stretched on a water bath, and baked to slides at $80^{\circ} \mathrm{C}$.

\section{Staining and imaging sections}

Sections were stained for 15 seconds with Toluidine blue O (Merck 1.15930; 1\% (w/v) Toluidine blue $\mathrm{O}$ in 1\% potassium tetra borate, washed 3 times for 5 min in water and enclosed in Euparal permanent mounting agent. Sections were imaged in a Nikon 80i microscope with 20x Plan Fluor 0.5 NA and 40x Plan Fluor 0.75 NA objectives and a DS Fi1 colour camera. When needed images were stitched using Image Composite Editor (V2.0.3.0, Microsoft research).

\section{Results}

Unexpectedly, when we excavated the termite mounds, we observed that there were two different types of structures: the normally described, irregularly shaped roundish nodules as well as distinctly differently shaped structures that could, however, easily be mistaken for nodules (Figure 1A). The shape of the latter was oval with a pointy top, and we hypothesised that these were true mushroom primordia (Figure 1B). Over three years we excavated 25 termite mounds, some of which in multiple years, adding 
up to 32 observations (Supplementary Tables 1 and 2). We noted potential primordia in six different mounds at seven observations. On each comb fragment with potential primordia, less than $20 \%$ of all fungal developmental structures were regular nodules.

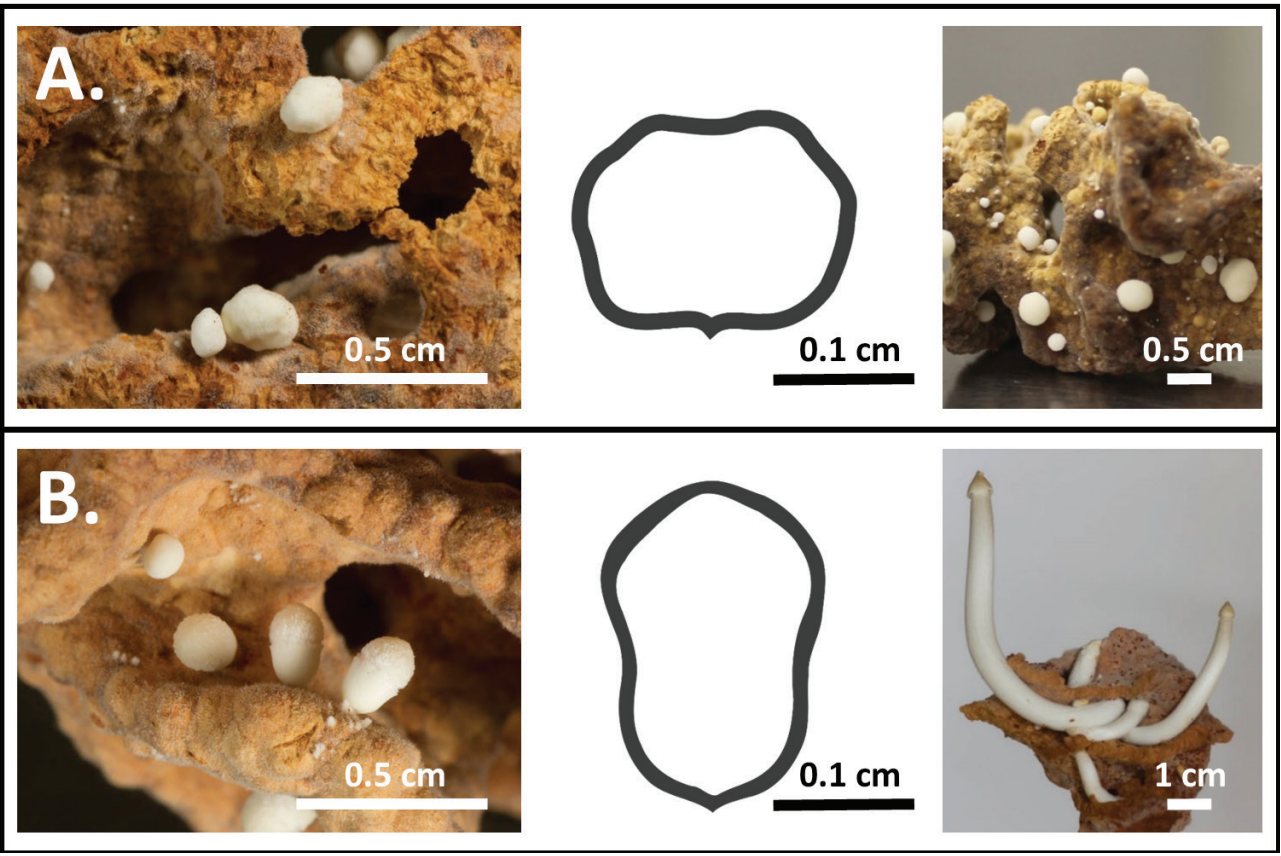

Figure 1| Two types of developmental structures found within mounds of M. natalensis: (A) Normal nodules (left), fungus-comb fragment, (middle) schematic drawing, and (right) fungus comb fragment incubated without termites for five days showing enlarged normal nodules. (B) Primordia (left), fungus comb fragment, (middle) schematic drawing, (right) mushrooms growing from primordia after four days of incubation without termites. The front of the fungus comb has been broken off, to fully show the mushroom stipes. Cap of the mushroom already shows the typical Termitomyces perforatorium (Heim 1977), i.e., the sharply pointed cap.

Of the 110 incubated fungus combs (Supplementary Table 1) 91 only displayed normal nodules or no nodules and 19 displayed potential primordia. On average each comb contains more than 10 nodules, meaning that we studied over 1100 developmental structures, of which about 900 were nodules and about 200 were potential primordia. When incubated in the absence of termites, none of the normal nodules developed into mushrooms, whereas six combs with potential primordia developed fully grown, spore-producing mushrooms. On all combs with potential primordia, there were also potential primordia that did not develop into mushrooms. These primordia were arrested at different stages of development and some of them turned brown and wilted. One comb fragment in our experiment, in which all normal nodules turned brown and 
wilted - taken from a mound with only normal nodules - produced primordia after 16 days of incubation. These primordia also developed into mushrooms.

The sections of potential primordia and their development showed that these developmental structures are indeed the true primordia of Termitomyces mushrooms (Figure 2B-Figure S1 A, B, C). In contrast, the sections of normal nodules did not show the hyphal alignment that is typical for mushroom formation (Figure 2A), but rather showed unorganised strings of ovoid asexual spores and larger spherical cells (Figure S1D, E). Moreover, the larger nodules that were studied after nine days did not develop mushroom features either.

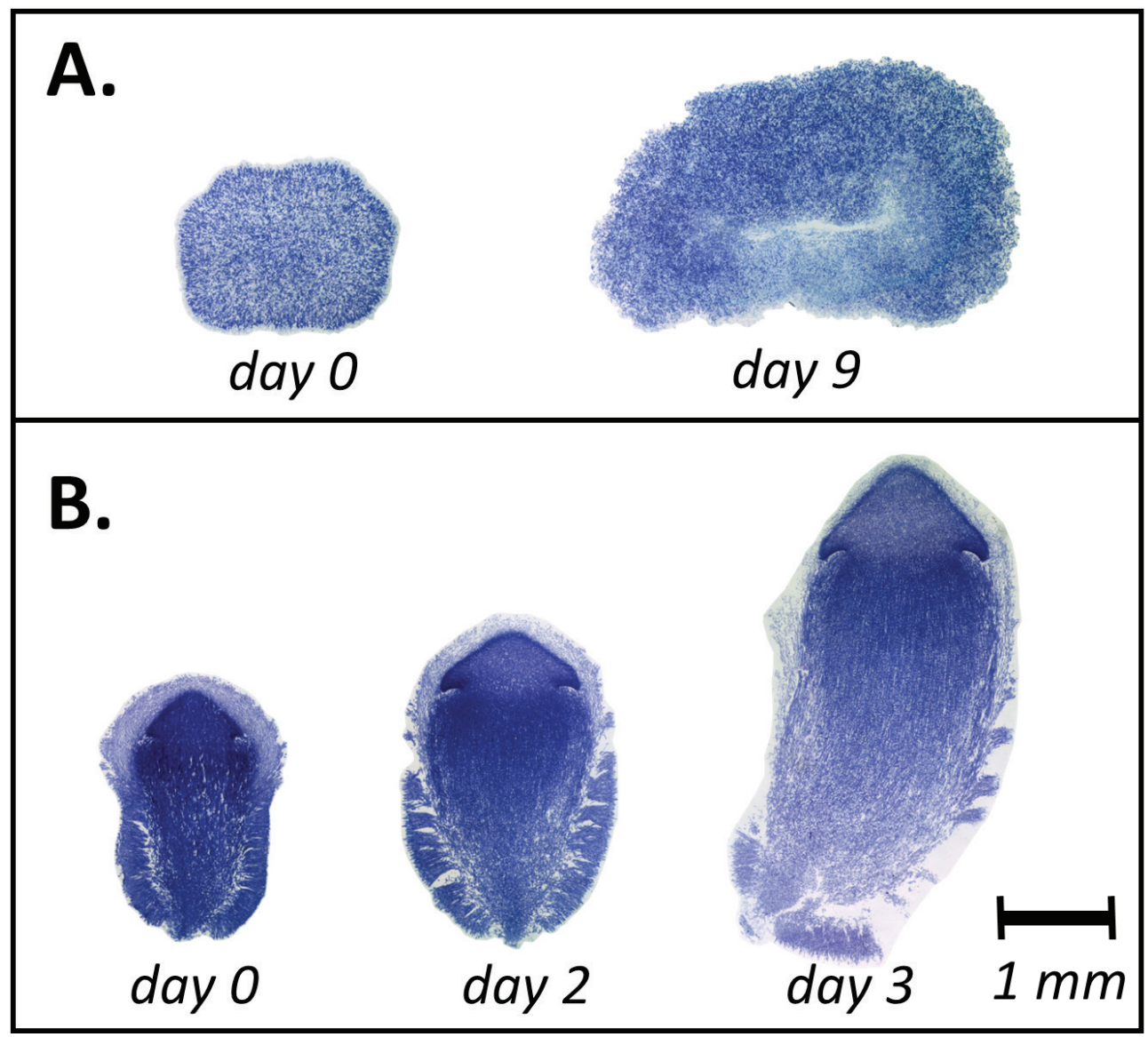

Figure 2 Images show Toluidine blue stained midplane sections of different developmental stages of (A) nodules versus (B) primordia after incubation in the absence of termites. 


\section{Discussion}

We tested the assumption that nodules are unripe mushrooms. We reject this assumption by showing that 1.) normal nodules do not develop into mushrooms and 2.) although Termitomyces primordia bear resemblance to nodules, they are macro- and microscopically different developmental structures. Our observations of normal nodules confirm earlier descriptions of normal nodules in other species (Heim 1977, Leuthold et al. 1989, Botha and Eicker 1991, Botha and Eicker 1992) and it is likely that our findings can be translated to all other Termitomyces species, as all known species make the nodules that are unique to this genus of fungi that are grown by termites (Petch 1906, Darlington 1994).

Although our results showed that nodules are not the initials of mushrooms, this does not prove or disprove that fruiting body formation in Termitomyces is actively suppressed by its host. Termitomyces primordia may, similar to nodules, be consumed by termites, but this remains to be tested. Behavioural studies in these termites are, however, notoriously difficult, as termites immediately repair open areas in their mounds. Li et al. (2016) have recently managed to set up a laboratory colony of Odontotermes formosanus, which opens up possibilities for future studies, including behavioural ones.

Regardless of whether primordia are or are not consumed, the triggers for primordia formation are unknown. We only observed primordia in $20 \%$ of the excavations and when we observed primordia on adjacent mound of the same species often did not have primordia. This indicates that there are factors within a colony that trigger or prevent primordia formation. We observed that combs that carried primordia were relatively mature in the sense that their colour was light, which is an indication of lignin breakdown and thus substrate depletion (Hyodo et al. 2000, da Costa et al. 2018). Also, we observed the formation of primordia on a fungus comb that had been incubated without termites for 16 days and was thus nutritionally depleted. Finally, it is known for other basidiomycete species that mushrooms can be formed in response to starvation (Kües and Liu 2000, Halbwachs et al. 2016, Sakamoto 2018). Therefore, we hypothesise that when fewer workers are present to maintain the fungus combs, some combs are left unattended and become nutritionally depleted because new substrate is no longer added. This nutritional depletion could, under the right environmental conditions, trigger formation of primordia. Our hypothesis is in line with the observation that Termitomyces microcarpus mushrooms are found on pieces of comb that are ejected from a termite colony (thus left unattended) and with the observation that mushrooms are sometimes found on dead, unattended colonies (Sieber 1983, Darlington 1994, Nobre et al. 2011a).

Analogously, in the convergently evolved obligate ant-fungus symbiosis, the conflict over symbiont dispersal is mitigated by ant control over symbiont dispersal (Mueller 
2002). If the ant fungus is grown on substrate that is poor in protein mushroom formation is triggered. However, if the fungus is grown on substrate that is too rich in protein, vegetative growth is hampered. Mushroom formation in the ant-fungus is suppressed by growing it on substrate that contains enough protein to prevent mushroom formation, but not so much that fungal growth is hindered (Shik et al. 2016).

\section{Acknowledgements}

We thank Z. Wilhelm de Beer, Bernard Slippers, Michael J. Wingfield, and the Forestry and Agricultural Biotechnology Institute, Pretoria, for hosting field work; Christine Beemelmanns, René Benndorf, Victoria L. Challinor, Benjamin H. Conlon, Haofu Hu, Nina Kreuzenbeck, Saria Otani, Kristine S.K. Pedersen, and Jane de Verges for help with excavations; Nicky P.M. Bos for help with excavations and photographs; Margo Wisselink, Lennart Van de Peppel, and Ben Auxier for help with excavations as well as critically commenting on the manuscript; Eric Bastiaans and Alexey A. Grum-Grzhimaylo for critical comments on the manuscript. We thank the Wageningen Light Microscopy Centre (WLMC) for technical support and equipment for sample processing and microscopy.

S.M.E.V. and D.K.A. were supported by The Netherlands Organisation for Scientific Research (ALW Open competition 824.01.002; VICI; NWO 86514007). R.R.d.C was supported by the CAPES Foundation, Ministry of Education of Brazil, Brasilia, Brazil (Grant BEX 13240/13-7). M.P. was supported by the Villum Kann Rasmussen Young Investigator Fellowship (VK10101). 


\section{Supplementary material}
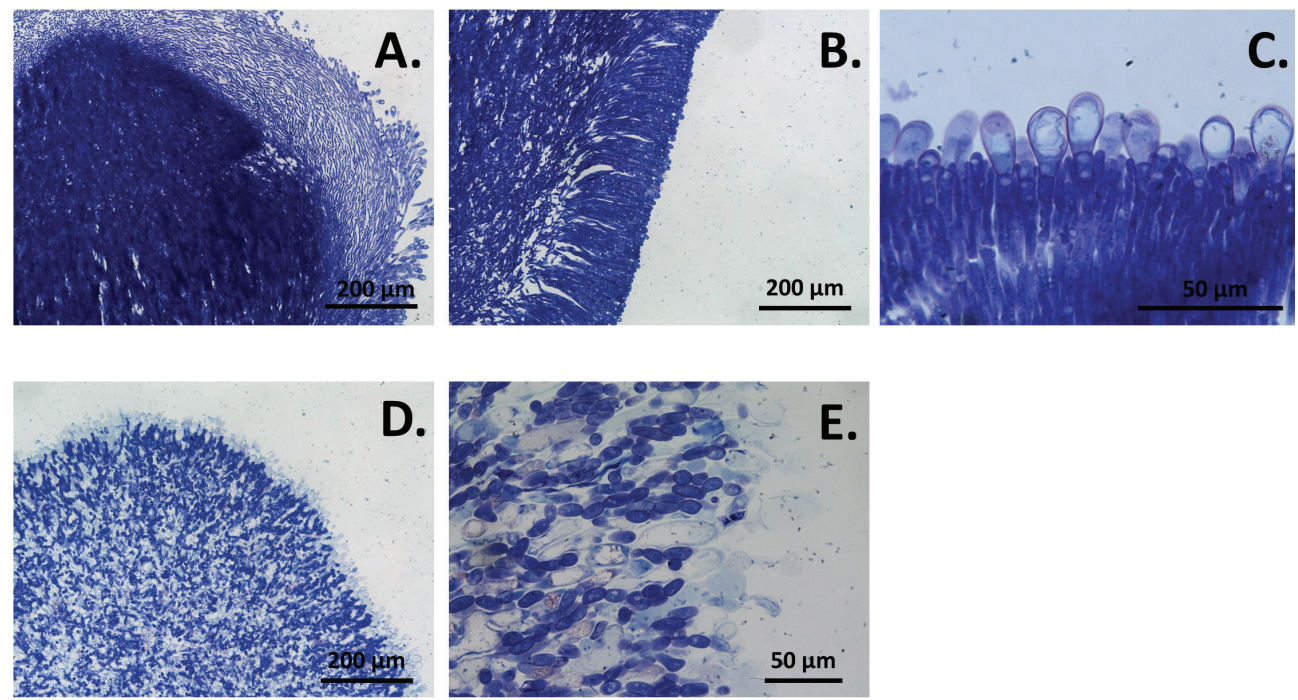

Supplementary Figure 1| Cross section enlargements of primordium (A, B) and primordium after 4 days of incubation without termites (C). (A) Primordia show the start of cap formation and (B) parallel orientation of hyphae at the outside of the stipe. After 4 days of incubation without termites (B) lamellae were present, yet basidia were not mature enough to observe spore formation. Cheilocystidia are clearly visible between basidia, which has been observed for many Termitomyces species (Heim 1977, van der Westhuizen and Eicker 1990, Pegler and Vanhaecke 1994, Wei et al. 2005). Cross section enlargements of nodules (D, E). Nodules show unorganised aggregation (D) of hyphae, ovoid conidiospores and larger cells (E). 


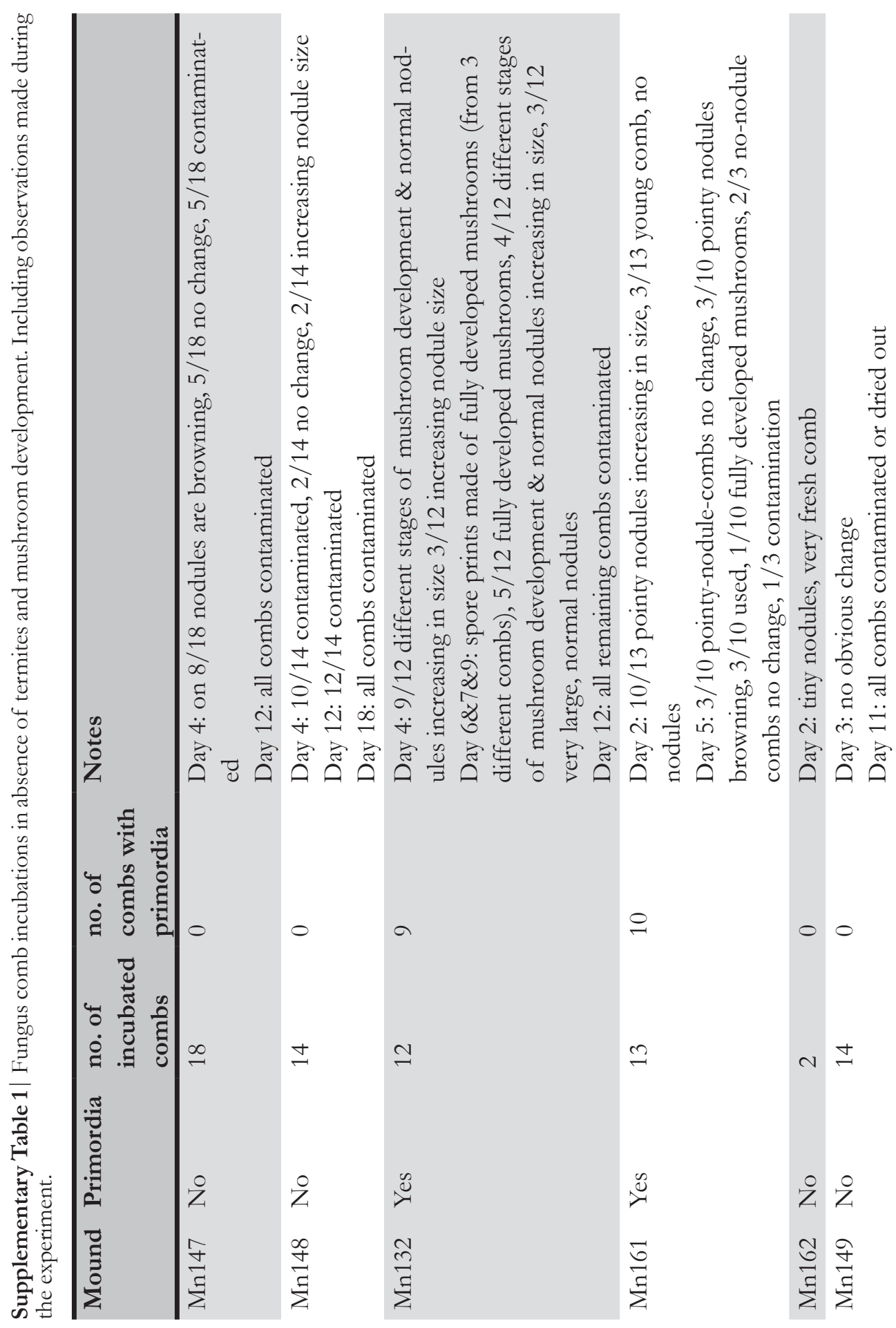




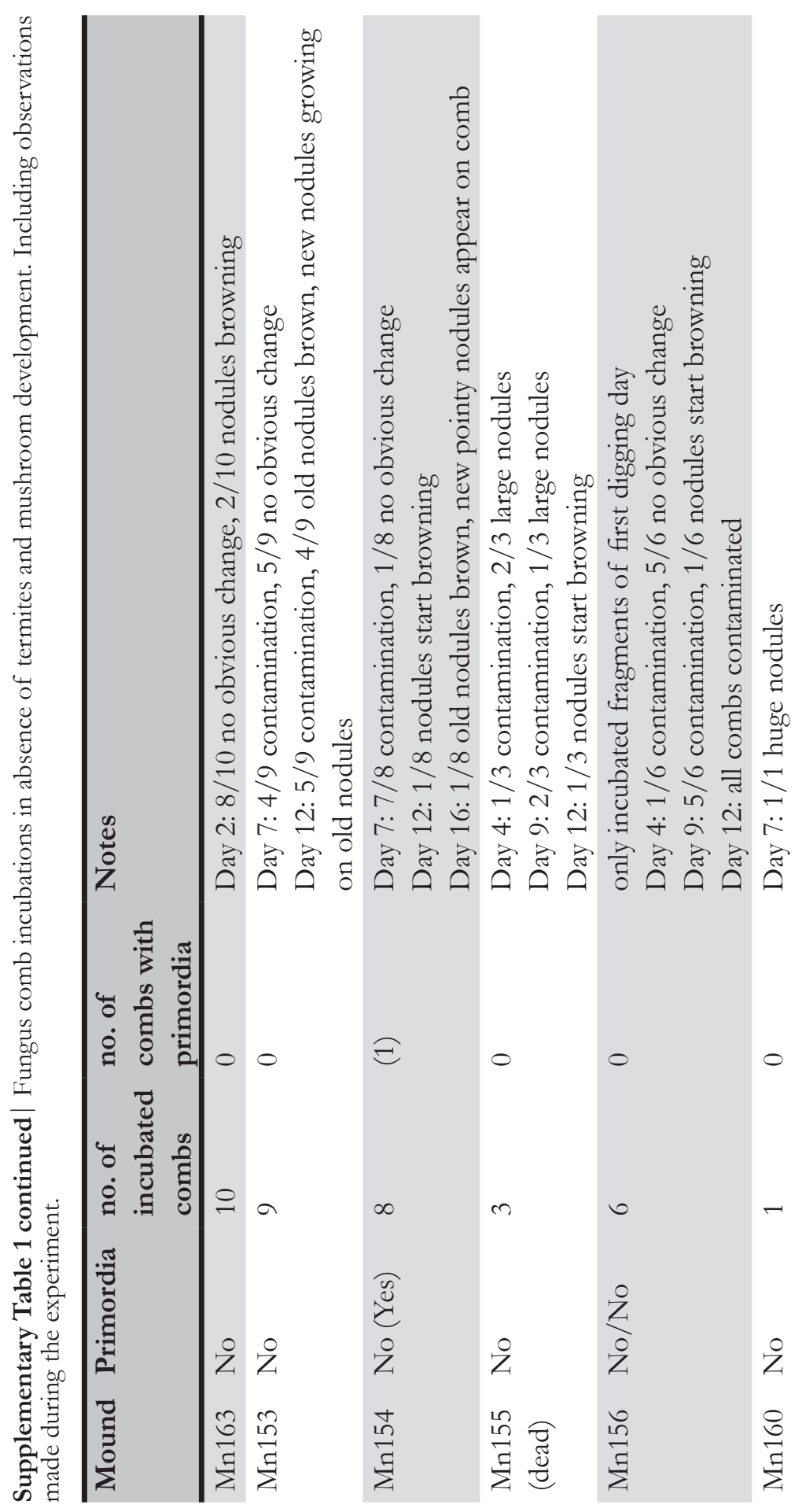


Supplementary Table $2 \mid$ Excavated $M$. natalensis termite mounds in January and February 2015, 2016 and 2018, including exact GPS locations and excavation dates. In total 25 mounds were dug of which five in multiple years, adding to 32 observations to show whether a mound contained pointy nodules (Yes), or only normal nodules (No). Mound Mn155 was dead at excavation, i.e., did not have a live queen and king, yet still contained uncontaminated fungus combs.

\begin{tabular}{|c|c|c|c|c|}
\hline \multirow[t]{2}{*}{ Mound } & \multirow[t]{2}{*}{ Location } & \multicolumn{3}{|c|}{ Primordia: } \\
\hline & & 2015 & 2016 & 2018 \\
\hline Mn147 & S24 40.478 E28 47.898 & No & - & - \\
\hline Mn148 & S24 40.509 E28 47.952 & No & - & - \\
\hline Mn132 & S24 40.484 E28 48.271 & Yes & Yes & No \\
\hline Mn161 & S24 39.668 E28 47.555 & Yes & - & - \\
\hline Mn162 & S24 39.693 E28 47.559 & No & - & - \\
\hline Mn165 & S24 39.724 E28 47.608 & - & No & - \\
\hline Mn166 & S24 39.666 E28 47.590 & - & No & - \\
\hline Mn173 & S24 39.694 E28 47.588 & - & Yes & - \\
\hline Mn187 & S24 40.434 E28 48.275 & - & - & No \\
\hline Mn188 & S24 40.512 E28 48.260 & - & - & Yes \\
\hline Mn149 & S25 43.698 E28 14.102 & No & - & - \\
\hline Mn163 & S25 43.761 E28 14.167 & No & - & - \\
\hline Mn168 & S25 43.708 E28 14.461 & - & No & - \\
\hline Mn169 & S25 43.666 E28 14.458 & - & No & - \\
\hline Mn190 & S24 40.512 E28 48.260 & - & - & Yes \\
\hline Mn153 & S25 44.492 E28 15.663 & No & No & - \\
\hline Mn154 & S25 44.581 E28 15.659 & No (Yes) & No & No \\
\hline Mn155 (dead) & S25 44.537 E28 15.659 & No & - & - \\
\hline Mn156 & S25 44.623 E28 15.655 & No & - & No \\
\hline Mn160 & S25 44.578 E28 15.645 & No & Yes & - \\
\hline Mn164 & S25 44.762 E28 15.434 & - & No & - \\
\hline Mn186 & S25 44.600 E28 15.648 & - & - & No \\
\hline Mn171 & S25 56.622 E30 35.869 & - & No & - \\
\hline 2004MN2.1ISWEPE & S26 48.898 E30 42.667 & - & No & - \\
\hline DUURMN2004-3-2 & S26 50.163 E30 30.490 & - & No & - \\
\hline
\end{tabular}




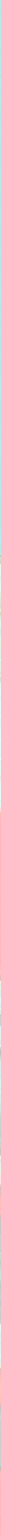




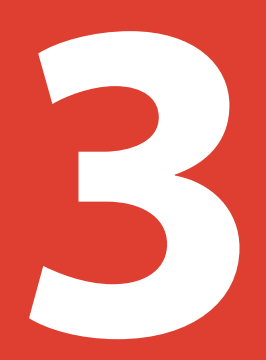

Can interaction specificity in the fungus-farming termite symbiosis be explained by nutritional requirements of the fungal crop?

\author{
Rafael Rodrigues da Costa*, \\ Sabine M.E. Vreeburg*, \\ Jonathan Z. Shik, Duur K. Aanen, \\ Michael Poulsen
}

Published in Fungal Ecololgy *contributed equally to this chapter 


\section{Abstract}

Fungus-growing termites are associated with genus-specific fungal symbionts, which they acquire via horizontal transmission. Selection of specific symbionts may be explained by the provisioning of specific, optimal cultivar growth substrates by termite farmers. We tested if differences in in vitro performance of Termitomyces cultivars from nests of three termite species on various substrates are correlated with the interaction specificity of their hosts. We performed single-factor growth assays (varying carbon sources), and a two-factor geometric framework experiment (simultaneously varying carbohydrate and protein availability). Although we did not find qualitative differences between Termitomyces strains in carbon-source use, there were quantitative differences, which we analysed using principal component analysis. This showed that growth of Termitomyces on different carbon sources was correlated with termite host genus, rather than host species, while growth on different ratios and concentrations of protein and carbohydrate was correlated with termite host species. Our findings corroborate the interaction specificity between fungus-growing termites and Termitomyces cultivars and indicate that specificity between termite hosts and fungi is reflected both nutritionally and physiologically. However, it remains to be demonstrated if those differences contribute to selection of specific fungal cultivars by termites at the onset of colony foundation. 


\section{Introduction}

Mutualisms are widespread in nature, with cooperation between species often providing entry into ecological niches that could not support either species alone (Moya et al. 2008). Yet, the interactions between mutualists vary from short-term co-existence to irreversible obligate symbiosis. In addition, there are varying degrees of interaction specificities; i.e., possible combinations of hosts and symbionts (Aanen et al. 2007). Vertical transmission of symbionts generally leads to a high degree of interaction specificity and co-evolution, whereas horizontal transmission typically leads to less specialised associations between symbionts (Bright and Bulgheresi 2010). Interaction specificity, however, not only depends on transmission mode. It is often observed that (metabolic) traits of a symbiont are lost because their functions become redundant as the other partner is reliably providing the resources (Visser et al. 2010, Ellers et al. 2012). Such reciprocal specialisation can favour obligate symbiotic partnerships and foster co-cladogenesis of symbionts, even in the absence of vertical transmission (Aanen et al. 2007).

An intriguing obligate symbiosis is that between a monophyletic group of termites (family Termitidae, subfamily Macrotermitinae) and basidiomycete fungi of the genus Termitomyces (Agaricomycetes, Lyophyllaceae), which originated ca. 30 million years ago in sub-Saharan Africa (Aanen et al. 2002, Aanen and Eggleton 2005, Roberts et al. 2016). Fungus farming enables the termites to utilise food sources they cannot digest themselves, as the fungi convert recalcitrant plant substrates into edible fungal biomass and accessible carbohydrates. In return, the termites shelter the fungus from unfavourable abiotic and biotic conditions (Wood and Sands 1978, Rouland-Lefèvre and Bignell 2002). There are about 330 described species of fungus-growing termites in 11 monophyletic genera (Aanen et al. 2002, Nobre et al. 2010), all farming Termitomyces fungal symbionts (Aanen et al. 2002, Roberts et al. 2016). Even though most termites acquire their symbionts horizontally (Figure 1A; De Fine Licht et al. 2006, Aanen et al. 2007, Nobre et al. 2010), there is a degree of co-cladogenesis between the termite and fungus phylogenies; groups of Termitomyces associate with specific termite clades (Figure 1B; Johnson et al. 1981, Sieber 1983, Darlington 1994, Aanen et al. 2002, Aanen et al. 2009). Yet, within these groups, large differences in interaction specificity are observed; some are highly specific, e.g., all Macrotermes natalensis studied so far associate with members from a single biological species of Termitomyces, whereas others have more diffuse co-evolutionary relationships; for example, most species of the genus Odontotermes associate with a broad range of Termitomyces lineages, which represent multiple species (Aanen 2006, De Fine Licht et al. 2006, Aanen et al. 2007).

To explain the observed co-cladogenesis and differences in interaction specificity between termite-fungus associations, several studies have proposed that the substrates 


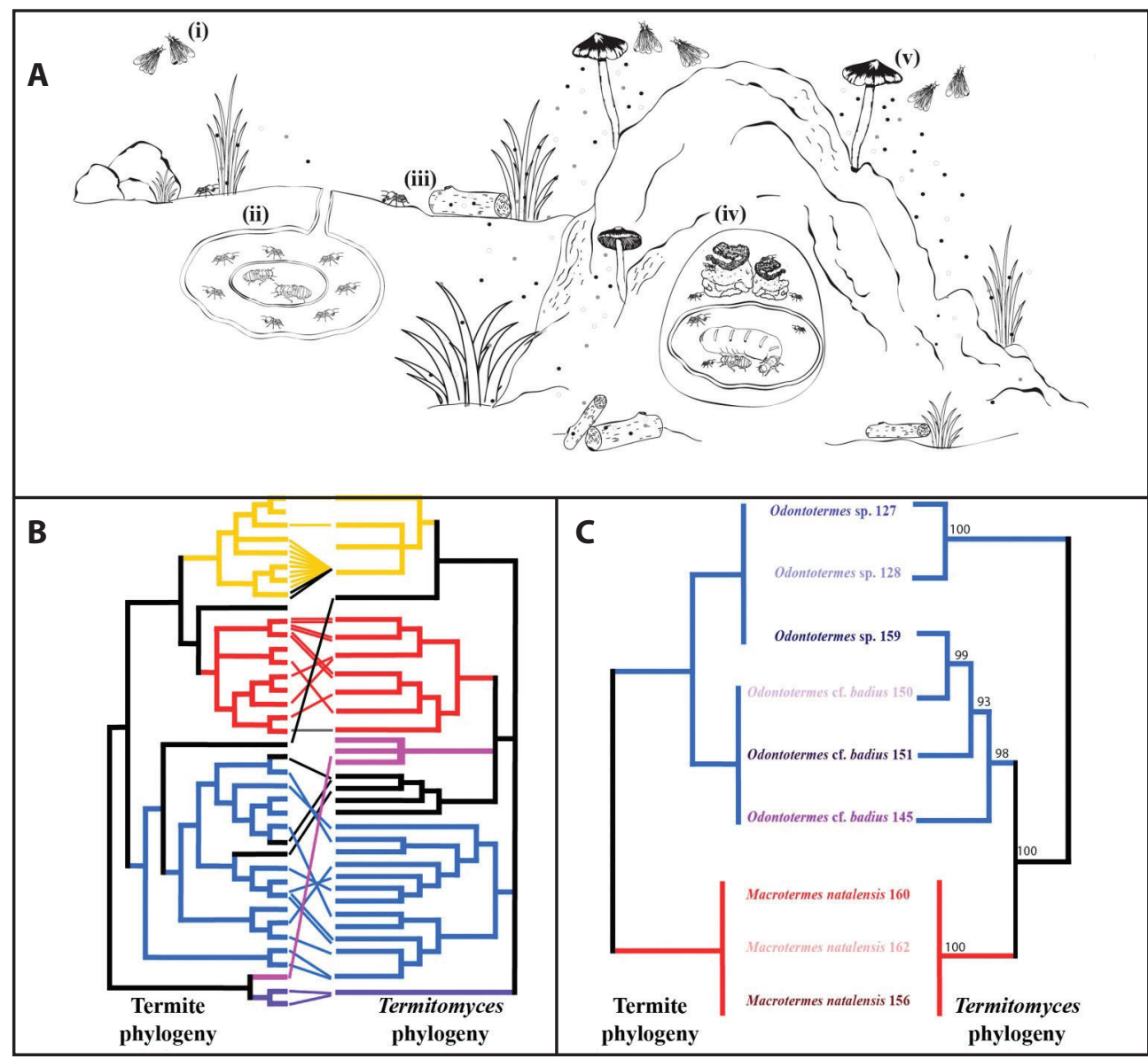

Figure 1| (A) Schematic of fungal/termite life cycle: (i) winged male and female termites (alates) leave a mature nest for the nuptial flight. (ii) They shed their wings and dig into the ground to establish a new colony. After few months the first generation of workers will build a chamber around the reproductive pair (former alates, now king and queen). (iii) This first generation of workers will leave the nest in order to obtain Termitomyces basidiospores from the environment along with plant substrate that will serve as growth substrate for the symbiotic fungus. (iv) Frequency-dependent selection between different Termitomyces strains assures that mature colonies maintain only a single fungus clone. Once the fungus is established as a fungus comb, it is nourished by workers with continuous plant substrate inoculation. (v) In mature nests, the fungus comb can produce fruiting bodies that emerge from the termite mound to release basidiospores into the environment. (B) Simplified consensus phylogenetic tree of fungus-growing termites species (left) and their fungal symbionts (right) (modified from Aanen et al. 2002). The lines in the centre indicate associations across termite and Termitomyces species; in blue the Odontotermes termites and their associated fungi, in red the Macrotermes termites and their associated fungi $(\mathrm{C})$ Termite (inferred) and Termitomyces (ITS based) phylogenies unrooted trees using cluster analysis (UPGMA algorithm with Jukes-Cantor distance measurement, and bootstrap support with 10,000 pseudoreplicates) for the three termite species and nine nests used in the study. Sequences were aligned using the CLC Genomics Workbench v9.5.3 (https://www.qiagenbioinformat-

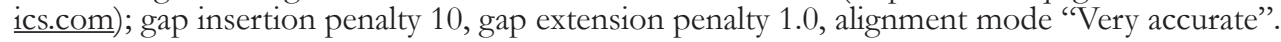


provided by termites play a role in the selection of a suitable fungal symbiont (Rouland-Lefèvre 2000, Nobre and Aanen 2012). The hypothesis is that the most specific associations have evolved most complementarity in the breakdown of plant biomass. According to this hypothesis, the less specifically cultivated Termitomyces, like the ones cultivated by Odontotermes spp., are expected to have a broader potential substrate range than very specifically cultivated Termitomyces, like the symbiont of $M$. natalensis. In addition, different termite species and genera collect different plant substrates, which could further select for association-specific metabolic capacities in the fungal symbionts (Grassé 1982, Dangerfield and Schuurman 2000, Johjima et al. 2006, Soleymaninejadian et al. 2014, da Costa et al. 2018).

Here, we explore the extent to which Termitomyces performance on different substrates is correlated with interaction specificity in the termite-fungus symbiosis. First, we generate a phylogeny of nine Termitomyces isolates to compare to previous work and confirm interaction specificities (Aanen et al. 2002, Aanen et al. 2007). Second, we test whether there are qualitative or quantitative differences in in vitro cultivar growth performance on 35 carbon sources between Termitomyces symbionts of the more specific Macrotermes natalensis and two less specific Odontotermes species. Third, as growth substrates are not expected to differ one-dimensionally (i.e., for carbon source only) between termite species, we use a geometric framework approach to generate nutritional landscapes by which we can visualise in vitro cultivar growth performance upon varying carbohydrate and protein concentrations and ratios simultaneously (Lee et al. 2008, Dussutour et al. 2010, Simpson and Raubenheimer 2012, Shik et al. 2016). Finally, we identify whether the observed growth patterns, separately for the carbon-source and the nutritional-landscape experiment, reflect the interaction specificity between the fungi and their hosts.

\section{Materials and methods}

\section{Termite collections and fungal isolations}

We studied Termitomyces fungi from mature colonies for which selection of the resident fungal cultivar has already taken place. Fungus comb samples were collected from nine fungus-growing termite nests in 2015 at three geographical locations South Africa (Table 1). Samples were collected from the termite species Odontotermes sp. (Od127, Od128 and Od159), Odontotermes cf. badius (Od145, Od150 and Od151), and Macrotermes natalensis (Mn156, Mn160 and Mn162), for which the termite species had previously been determined (Table 1; Otani et al. 2014, da Costa et al. 2018). Mature, nodule-containing parts of the fungus comb were collected, placed into plastic bags and taken to the laboratory. Termitomyces fungal nodules were picked from clean parts of the fungus comb (no visible soil particles under a binocular microscope) with a sterile needle and placed 
on Petri plates with Malt Yeast Agar (MYA: $20 \mathrm{~g}$ malt, $2 \mathrm{~g}$ yeast extract, $15 \mathrm{~g}$ agar in $1 \mathrm{~L}$ distilled water). Fungal growth was monitored daily to check the purity of the isolates.

\section{Termitomyces barcoding and phylogenetic analysis}

Termitomyces DNA was isolated using a Cetyltrimethylammonium Bromide (CTAB) extraction. For isolates from mounds Od128, Od145, Od150, Od151 and Mn162, part of the nuclear ribosomal region, including both internal transcribed spacer (ITS) regions and the 5.8S ribosomal RNA (ITS1, 5.8S and ITS2), were amplified and sequenced using ITS1 and reverse primer ITS4 (White et al. 1990). Because Termitomyces is present in termite mounds as a heterokaryon, i.e., with two separate haploid nuclei, the total DNA of each isolate can contain two different copies for each region of the genome, which was the case for at least one of the two ITS regions in the fungal isolates of mounds Od159, Mn156, Mn160 and Od127. Further, if a length mutation exists between the two different copies of a genomic region, Sanger sequencing will fail. Therefore, we used both forward primers ITS1 and ITS3 and reverse primers ITS2 and IST4 (White et al. 1990) to obtain most of the ITS sequences. We obtained both ITS regions, but not the 5.8S region for Termitomyces from mound Od159. For Mn156 and Mn160 we obtained ITS1 and part of ITS2, and for Od127, part of ITS1, and part of ITS2. Sequences have been deposited to GenBank (MG283253-MG283261; Table 1). Sequences were aligned using the CLC Genomics Workbench v9.5.3 (https://www.qiagenbioinformatics.com); gap insertion penalty 10, gap extension penalty 1.0, alignment mode "Very accurate". An unrooted tree was obtained by cluster analysis using the UPGMA algorithm with Jukes-Cantor distance measurement, and bootstrap support was assessed using 10,000 pseudoreplicates (Figure 1C). Using BLASTn, the ITS sequences were compared to haplotypes obtained in a study of South African Termitomyces by Aanen et al. (2007).

\section{Single nutrient assay: performance on different carbon sources}

To determine biomass production of Termitomyces strains on different carbon substrates, we used a minimal medium developed for Schizophyllum commune (Supplementary Table 1; Dons et al. 1979), supplemented with $300 \mathrm{mg}$ Urea/L, where glucose was replaced by one of 35 carbon sources as described at www.fung-growth.org, without birch wood xylan and oat spelt xylan that were no longer available and with chitin added (de Vries et al. 2017). The use of this specific set allows for comparison to other studies gathered in the FUNG-GROWTH database (e.g; Benoit et al. 2015, de Vries et al. 2017). Sterilised polycarbonate membranes (Profiltra, 0.1 pore size, $76 \mathrm{~mm}$ diameter, Almere, The Netherlands) were placed in each Petri dish to facilitate fungal biomass collection and weighing after incubation. Termitomyces strains were cultured for 15 days on MYA before roughly the same amount of fungal material from each strain was harvested and added to each of six Eppendorf tubes containing $750 \mu \mathrm{l} 0.6 \%$ saline. The suspensions were subsequently pooled in a $12 \mathrm{ml}$ tube and vortexed. Ten $\mu \mathrm{L}$ of this hypha/spore 


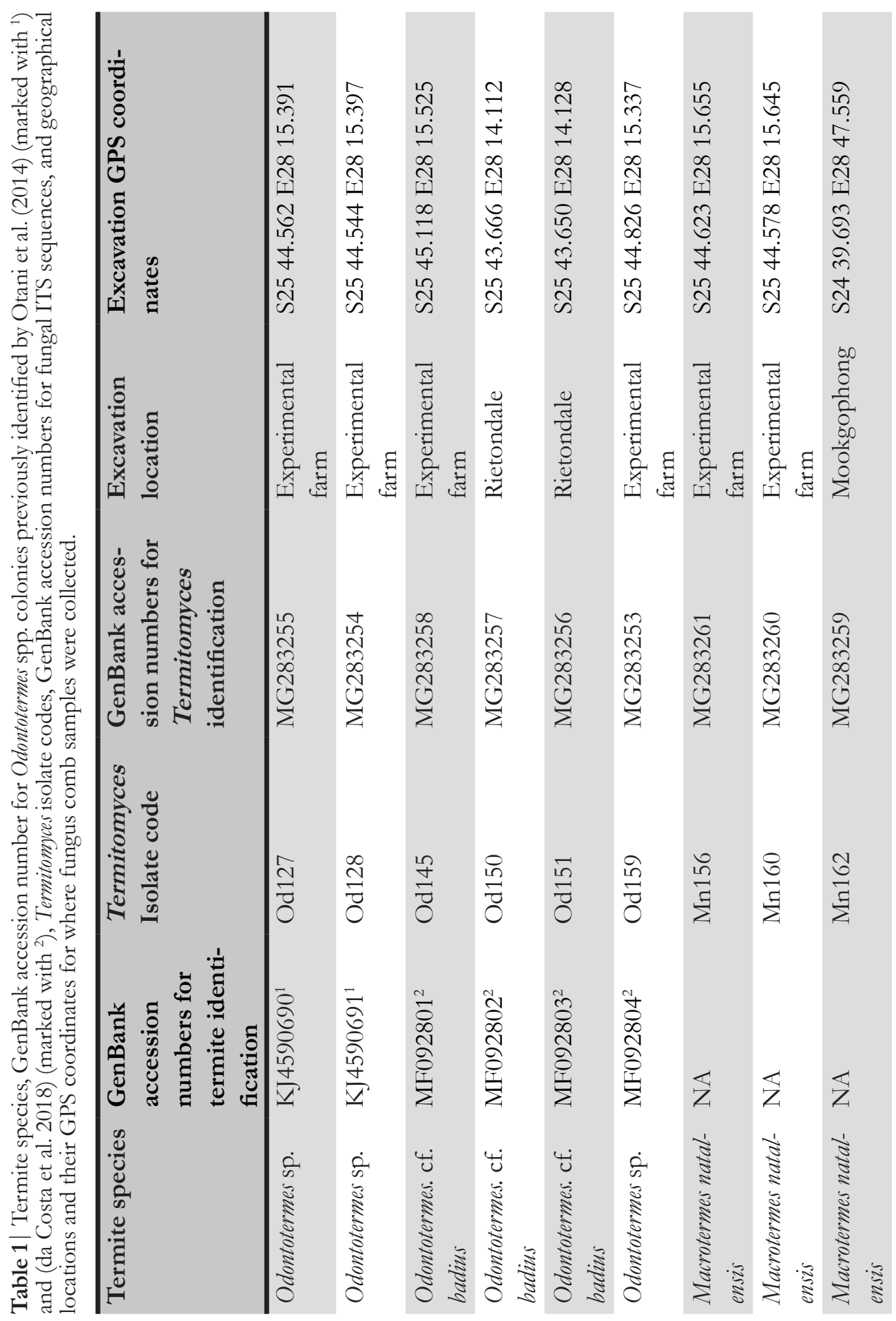


suspension was used as the inoculum. Three inoculates were placed on one plate; three replicate plates were inoculated per fungal strain per carbon substrate (total of nine replicates per fungus, per carbon source). The plates were incubated for 21-31 days (Supplementary Table 2), after which, per Petri dish, biomass was collected and weighed ( $\mathrm{g}$ wet weight). Contaminated plates were discarded (18\% out of 945 plates). Plates without membranes were incubated and photographed for visual inspection (Supplementary Figure 1). Area could, however, not be evaluated, because of a small number of replicates. Principal Component Analysis (PCA) was performed on the average biomass per strain, per carbon source ( 35 measurements per strain) in R v. 3.3.2 (R Core Team 2019) using the FactoMineR package (Lê et al. 2008). Contamination led to missing data points, for which values were estimated using missMDA in R (Josse and Husson 2016). The carbon sources that contributed the most to the PCA patterns were evaluated by their cumulative percentage of variance explained, loading values, and eigenvalues (Supplementary Table 3, and 4).

\section{Geometric framework assay: performance across protein:carbohydrate ratios and concentrations}

Termitomyces cultivar biomass formation and radial growth was assessed on media with nine protein:carbohydrate $(\mathrm{P}: \mathrm{C})$ ratios $(1: 9,1: 6,1: 3,1: 2,1: 1,2: 1,3: 1,6: 1$, and 9:1) in four different concentrations (8, 32, 56 and $80 \mathrm{~g} / \mathrm{L}$ protein + carbohydrate) (Lee et al. 2008, Dussutour et al. 2010, Shik et al. 2016). The mixtures of carbohydrate and protein were prepared in $250 \mathrm{~mL}$ distilled water and $4 \mathrm{~g}\left[1.6 \%\right.$ (wt:vol)] agar and autoclaved at $121^{\circ} \mathrm{C}$. Carbohydrates were provided by even parts glucose and starch (Sigma-Aldrich, St. Louis, MO, USA). Protein was provided by even parts bactopeptone, trypticase peptone, and bactotryptone (Sigma-Aldrich, St. Louis, MO, USA). Micronutrients were provided by crushed vitamins added at $2 \%$ of the cumulative mass of protein and carbohydrates (Centrum) (modified from Shik et al. 2016; for full media recipe, see Supplementary Table 5). Sterilised polycarbonate membranes (Profiltra, 0.1 pore size, $76 \mathrm{~mm}$ diameter; Almere, The Netherlands) were placed in each plate to facilitate fungal biomass retrieval after incubation.

Prior to inoculation, Termitomyces strains were cultured for 20 days on PDA plates, and they were harvested and inoculated as described above, except that each plate was inoculated with only a single inoculum. After 24 days, the Petri dishes were photographed (Supplementary Figure 2); contaminated plates excluded (1.3\% of the 972 plates). The images were used to measure growth area in ImageJ (NIH Image, v.1.50g). After photographing, fungal biomass was harvested and weighed. To analyse differences in biomass formation for different $\mathrm{P}: \mathrm{C}$ ratios among fungal strains, a PCA was performed in $\mathrm{R} v$. 3.3.2 (R Core Team 2019) using the FactoMineR package (Lê et al. 2008). Cumulative percentage variance, loading and eigenvalues were calculated to establish which ratios 
of P:C contributed the most to the separation observed in the PCA (Supplementary Table 6 and 7 ).

To visualise Termitomyces growth, we generated P:C landscapes upon which we mapped variation in fungal area $\left(\mathrm{cm}^{2}\right)$ and biomass (g wet weight) after 24 days. We did this using the "fields" package in v.2.14.0 R to generate non-parametric thin-plate splines (Lee et al. 2008, Dussutour et al. 2010), setting topological resolution of response surfaces to $\lambda$ $=0.001$ as a smoothing parameter (Shik et al. 2016). We tested whether colony identity influenced growth, performing GLM analyses (proc GLM, SAS V9.3) for each of the three fungal strains (i.e., fungal strains from nests of a given termite host species). Models for biomass and area included the factors protein, carbohydrate, protein x carbohydrate, protein squared, carbohydrate squared colony identity, and interactions between colony identity and each of the protein and carbohydrate effects. Colony identity was never a significant factor (Supplementary Table 8), so we generated performance landscapes based on strain-level means. For subsequent interpretation of P:C landscapes, we used least-square regressions based on strain-level means with both linear and quadratic terms to evaluate the main and interactive effects of protein and carbohydrates on performance (Supplementary Tables 9, 10, and 11).

\section{Results}

\section{Termitomyces barcoding and phylogeny}

All fungal isolates from $M$. natalensis had identical ITS sequences, apart from the occasional SNP between the two nuclei of the heterokaryons, while there were larger differences in ITS sequence and length between fungal isolates from Odontotermes spp. All the sequences were compared to sequences available on GenBank (https://www.ncbi. nlm.nih.gov/genbank/). Sequences of two isolates from Odontotermes sp. (Od127 and Od128) only differed in two insertions/deletions, and they were most similar to a fungal strain previously isolated from Odontotermes latericius (Figure 1C; Aanen et al. 2007). Odontotermes sp. (Od159) was most similar to an isolate from O. cf. badius (Od150) and both were most similar to an isolate from O. latericius (Figure 1C; Aanen et al. 2007). The other two O. cf. badius isolates (Od145 and Od151) were most similar to fungal strains previously isolated from the same termite species (Aanen et al. 2007). The placement of the strains in the phylogeny we generated based on our ITS sequences, corroborated the phylogeny of the most similar strains generated by Aanen et al. (2007).

\section{Termitomyces performance across single carbon sources}

Contrary to the hypothesis that the less specifically cultivated Termitomyces are able to grow on a broader range of substrates, we did not observe qualitative differences between Termitomyces strains in growth on different substrates. We did, however, find con- 
sistent quantitative differences between Termitomyces strains associated with $M$. natalensis and Odontotermes spp. The PCA showed that the Termitomyces strains associated with $M$. natalensis clustered together and were separated from Odontotermes spp. strains (Figure 2A). Loading values of the PCA indicated that the main contributors to the separation in the first principal component were cotton seed pulp, cellulose, alfalfa meal, Arabic gum and soybean hulls (negative loading values), and by $\mathrm{D}$-galacturonic acid, D-glucose, L-arabinose, casein and soluble starch (positive loading values) (Supplementary Table 3). The main contributors to the separation in the second component were D-glucuronic acid and lignin (negative loading values), and apple pectin and beechwood xylan (positive loading values) (Supplementary Table 3). There was no effect of incubation time on biomass formation (correlation analysis: $y=0.0047 x+0.028, R^{2}=0.0043$ ).

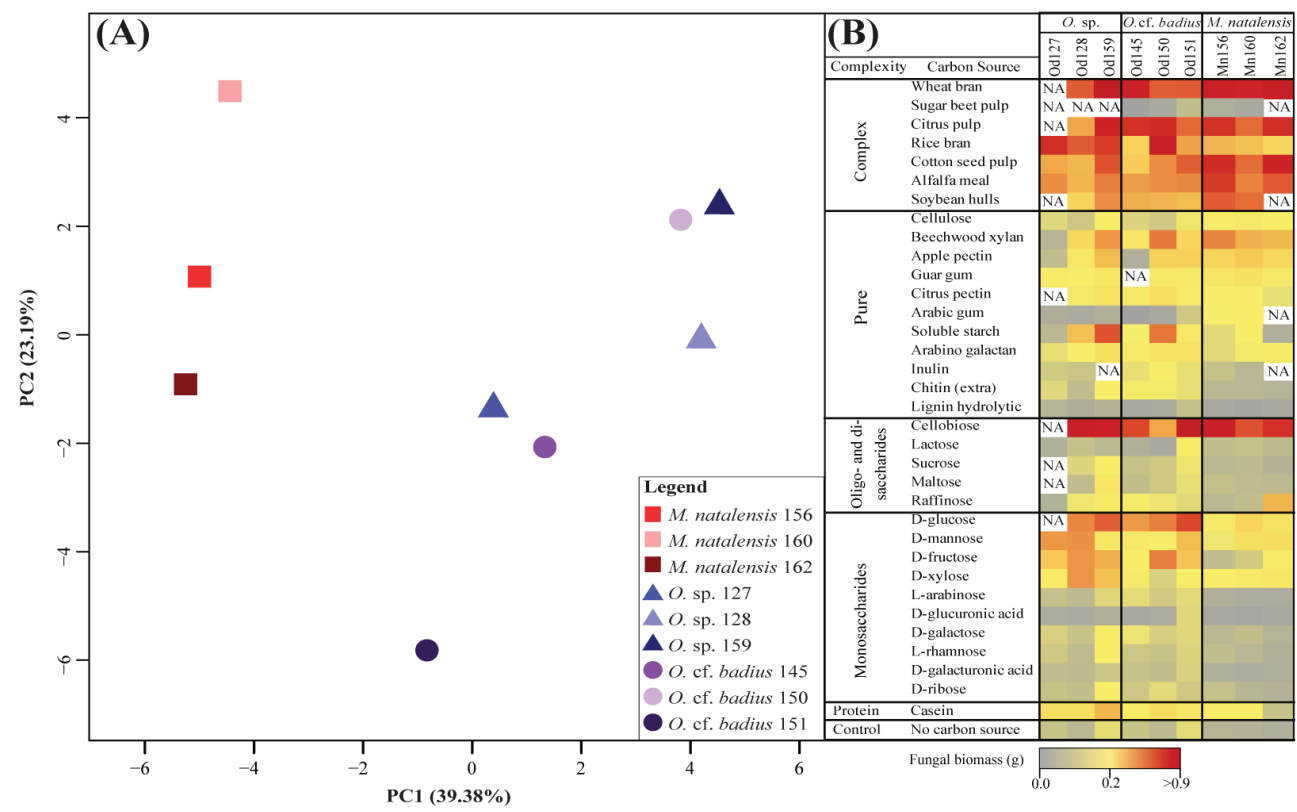

Figure 2| (A) PCA plot of biomass formation patterns on different carbons sources. Each dot represents the average biomass production pattern of a single Termitomyces strain (showed in B). (B) Heatmap showing average $(\mathrm{n} \leq 9)$ biomass formation by Termitomyces strains on different carbon sources. The colour scale ranges from grey for the lowest values, yellow for intermediate and red for the highest values. NA means that all plates were contaminated, i.e. missing data.

Overall, Termitomyces strains grew best on complex substrates such as wheat bran, citrus pulp, rice bran, cottonseed pulp, alfalfa meal and soybean hulls, and on the disaccharide cellobiose (Figure 2B; Supplementary Table 12). Sugar beet pulp was the only complex substrate with limited Termitomyces growth. Among the monosaccharides, D-glucose was the only one that allowed substantial biomass formation across all strains. None of the strains formed substantial biomass on lignin. Also, little biomass was formed on 
sucrose, even though some biomass was formed on D-glucose and D-fructose, the monosaccharides that build sucrose. Odontotermes spp. strains grew slightly better on D-glucose, D-fructose, and chitin than $M$. natalensis strains, while $M$. natalensis strains shown slightly better growth on Arabic gum (Figure 2B).
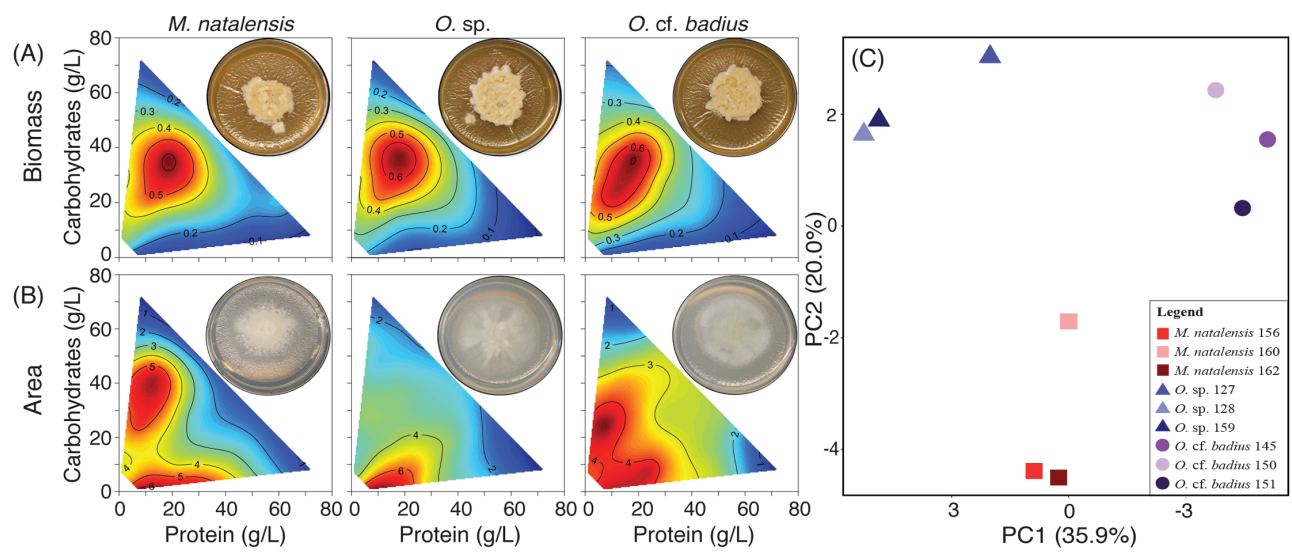

Figure 3| Nutritional landscapes mapping the performance of Termitomyces cultivars on different ratios (1:9 to 9:1 P:C) and concentrations (8 to $80 \mathrm{~g} / \mathrm{L}$ ) of protein and carbohydrates in nutritionally defined artificial media. (A) Red areas indicate P:C blends that maximised biomass production in grams (top row) and (B) radial growth area in $\mathrm{cm} 2$ (bottom row) and dark blue represent minimum values for these variables. Coloured isoclines increase to maximal dark red values of $>0.6 \mathrm{~g}$ of fungal biomass in (A) and $>5 \mathrm{~cm} 2$ fungal area in (B) measured after 24 days. Fungal cultivars are from colonies of $M$. natalensis (left), Odontotermes sp. (middle) and Odontotermes cf. badius (right) ( $\mathrm{n}=3$ colonies per species). All response surface regressions were significant (Supplementary Tables 8-11). (B) Principal component analysis on the biomass formation patterns on different P:C ratios and concentrations. Each dot represents the average biomass formation production pattern of a single Termitomyces strain. Pictures of plates represent the best performance for biomass (A) and area (B). For the full results, see Supplementary Figure 2.

\section{Cultivar performance across protein:carbohydrate landscapes}

In contrast to the results obtained on biomass formation patterns on different carbon sources, the PCA on biomass formation patterns on different P:C ratios and concentrations indicated clustering based on termite species (Figure 3C). Fungal cultivar performance is maximised by specific blends of protein and carbohydrates (Supplementary Tables 13 and 14), with strains exhibiting carbohydrate-biased 'bulls eyes' for maximal biomass production around concentrations of $35 \mathrm{~g} / \mathrm{L}$ carbohydrates and $20 \mathrm{~g} / \mathrm{L}$ protein (Figure 3A; Supplementary Tables 9, 10 and 11). The effects of carbohydrates on fungal biomass production interacted with protein availability (i.e., quadratic terms for C were significant for each of the strains; Supplementary Table 11), indicating that single nutrient assays of cultivar performance (e.g., the carbon source test) need to be interpreted with caution. Visualised on the performance landscapes, the same carbohydrate concentration (i.e., a horizontal line extending from the y axis at $32 \mathrm{~g} / \mathrm{L}$ ) that was 
associated with the highest cultivar biomass when present in slight abundance relative to protein (i.e., red bullseye at 1:2 to 1:3 P:C) was also associated with low biomass production at higher protein levels (i.e., blue areas to the right of red bullseyes, with diets $>2: 1$ P:C) (Figure 3A).

The P:C ratios and concentrations that maximised growth area differed visually from those for biomass formation (Figure 3A) and statistically, as the quadratic term for $\mathrm{P}$ was significant in each of the radial area regressions (but not in the biomass regressions, Supplementary Table 11). Regions of maximal growth area also appeared to differ across Termitomyces strains from different termite species (Figure 3B). The strains from $M$. natalensis showed a P:C diet maximum for radial growth that was comparable to the maximum for biomass formation (between 1:2 and 1:3, and between 32 and 56 $\mathrm{g} / \mathrm{L}$ ) and a second at P:C 3:1 and 6:1 at $8 \mathrm{~g} / \mathrm{L}$ (Figure 3B). The strains from O. cf. badius were similar to $M$. natalensis, while Odontotermes sp. strains only displayed one maximum (P:C 3:1-9:1 at $8 \mathrm{~g} / \mathrm{L}$ ) (Figure 3B).

\section{Discussion}

\section{Termite-fungus interaction specificity and its associations with Termitomyces growth}

Our phylogeny of fungi isolated from $M$. natalensis and two species of Odontotermes revealed patterns of interaction specificity consistent with previous work (Aanen et al. 2002, Aanen et al. 2007), demonstrating that M. natalensis colonies associate with a single Termitomyces species, while symbionts associated with Odontotermes spp. are genetically more variable and without one-to-one termite-to-fungus species relationships. Our in vitro assays suggest that Termitomyces nutritional requirements could contribute to interaction specificity with termite hosts. The patterns of biomass formation on different carbon sources separated $M$. natalensis from Odontotermes spp. strains, but not Odontotermes spp. from each other (Figure 2B), consistent with higher specificity in $M$. natalensis-Termitomyces interactions. Yet, the in vitro growth patterns in our two-factor geometric framework experiment, also separate the strains of the two Odontotermes species.

\section{Termitomyces performance of different carbon sources}

The termite species included in this study primarily harvest decaying wood and leaf litter (Wood and Sands 1978) and it was thus unsurprising that all Termitomyces strains performed well on complex carbon sources (Visser et al. 2011, Poulsen et al. 2014). Our findings do, however, suggest that $M$. natalensis should preferentially collect leaf litter and dead wood that are rich in cellulose, while Odontotermes should preferentially collect substrates richer in mono-, di- and oligosaccharides (Figure 2B). Substrate preference is generally poorly understood in fungus-farming termites, but variation does indeed ex- 
ist: fresh leaves (e.g., Macrotermes mülleri, Macrotermes ivorensis, Macrotermes michaelseni, and Odontotermes formosanus; (Grassé 1982, Dangerfield and Schuurman 2000, Soleymaninejadian et al. 2014)), grass stalks (e.g., Macrotermes bellicosus and Macrotermes subbyalinus; (Grassé 1982)), roots (e.g., M. michaelseni, Ancistrotermes sp., and Odontotermes sp.; (e.g., M. michaelseni, Ancistrotermes sp., and Odontotermes sp.; Dangerfield and Schuurman 2000)), and mammal dung (e.g., Odontotermes sp., O. cf. badius, M. natalensis, M. michaelseni; (Dangerfield et al. 1998, da Costa et al. 2018)). Consistently, a recent comparison found higher expression of cellulases in $M$. natalensis than in Odontotermes sp. fungus combs ( $\mathrm{da}$ Costa et al. 2018), and Johjima et al. (2006) found that pectinases and hemicellulases were expressed more than cellulases in Termitomyces associated with Macrotermes gilvus, which harvests grasses and leaves.

All Termitomyces strains formed more biomass on cellobiose than D-glucose, the building block of cellobiose. This may appear counterintuitive, since the degraded entities that are taken up by the fungus are monosaccharides (Allaway and Jennings 1970) but is likely because we standardised carbon source concentrations and not carbon content, and cellobiose has more total carbon (and hence higher energetic value) than D-glucose. This appears to be unproblematic for our interpretations, as there was no overall correlation between the number of carbon molecules and biomass formed for the mono-, di- and oligosaccharides for which we could estimate energetic values $(y=0.003 x+$ $\left.0.038, \mathrm{R}^{2}=0.019\right)$. Biomass formation is thus more likely associated with metabolic adaptations to certain carbon sources, consistent with the higher expression of enzymes targeting these carbon sources (e.g., cellobiohydrolases) in fungus combs (da Costa et al. 2018). Cellobiohydrolases convert cellobiose to monosaccharides, which likely leads to increased glucose levels in guts of old workers at the final step of plant decomposition (da Costa et al. 2018), and consistent with gut bacteria putatively being breaking down and assimilating simple sugars (Poulsen et al. 2014). The potential importance of nutrient co-limitation (evident from the geometric framework experiment) represents a caveat to the above interpretation, since carbohydrate-mediated cultivar growth depends on protein availability.

Termite species forage on different substrates in different geographical locations (da Costa et al. 2018), so Termitomyces growth likely varies in response to the availability of substrates in given habitats. Analyses of a Termitomyces genome from $M$. natalensis indicate that the cultivar should be able to decompose most complex polysaccharides (Poulsen et al. 2014). This implies that the substrates should not govern symbiont selection alone; however, variation in forage availability between habitats could affect what enzymes are most expressed. Further, because multiple fungal symbiont species associate with multiple Odontotermes species, genome content and enzymatic capacity variation between symbionts could be larger and allow termite host species to utilise a wider 
range of substrates. Additional -omics approaches will be needed to fully characterise the standing variation and the relative roles of genome differences vs. gene expression differences between Termitomyces species.

\section{Fungal performance across gradients of P:C blends and termite species-specif- ic farming strategies}

The geometric framework provides a powerful graphical approach for visualising how foraging diverse organisms from slime moulds (Dussutour et al. 2010) to humans (Simpson and Raubenheimer 2012) prioritise competing nutritional requirements when confronted with imbalanced resources. We used this approach to visualise nutritional landscapes and test for nutritional specificity differences between cultivar strains. While cultivars exhibit nearly identical nutritional P:C targets for maximum biomass production (Figure 3A), they appear to exhibit strain-specific targets for growth area (Figure 3B). Thus, if nutrients act as a filter among competing strains in incipient nests, they likely favour cultivars best able to exploit nutrients through rapid radial growth than those able to acquire most biomass over time. This may be driven by heightened starvation responses, with low macronutrient concentrations (especially carbohydrates) inducing extensive radial growth (cf., Boddy 1999, Boswell et al. 2002, Heaton et al. 2010).

An important next step will be to define nutritional mixtures present in each of the resources harvested by termite colonies in nature. By overlaying these maps of nutrient availability on growth maps, the degree of overlap between the 'realised niche' (nutrients actually provided by termites) and the 'fundamental niche' (resources that maximise cultivar performance) can be evaluated (Raubenheimer 2011). A reasonable hypothesis is that the substrates available to termite foragers in nature have low overall protein concentrations and low nutritional variability. For instance, the carbohydrate bias of optimal biomass formation may reflect that dead wood substrates can contain less than $0.5 \%$ nitrogen (LaFage and Nutting 1978) and fungus combs contain 1.9\% and 41\% nitrogen and carbon, respectively (Arshad and Schnitzer 1987). The P:C cultivar growth has additional eco-evolutionary importance, since the existence of nutritional 'bullseyes' implies that termites may stand to benefit by providing cultivars with a specific blend of nutrients (i.e. to target the fundamental niche of their cultivar). This in turn implies a basis for selection of sensory mechanisms in the termite host, and potentially among termite species growing different cultivar species, to identify and select those nutritional blends.

\section{Termite colony foundation and forage use may affect fungal cultivar selection}

The ecological dynamics by which fungus-growing termites select their symbionts during early phases of colony development remain unknown. One possibility is that the first termite workers selectively pick up Termitomyces spores from the species they normally associate with, along with plant substrate to start the fungal comb. This would, 
however, require recognition capabilities in the termites (Grassé and Noirot 1958, Sands 1960, Sieber 1983, Aanen et al. 2002). Sieber (1983) demonstrated that provisioning of Termitomyces spores to incipient Odontotermes montanus and M. michaelseni laboratory colonies allowed for the colonisation of a primordial fungus comb (comprised of soil) by Termitomyces. However, these experiments did not involve testing whether only specific fungal species were collected. Alternatively, multiple Termitomyces species may randomly be collected by the first termite workers if spores are brought in with plant forage coincidently. In this case foraged plant material could contribute to drive the subsequent competitive selection within the nest prior to the establishment of a monoculture.

Our findings provide support to the interaction specificity between fungus-growing termites and Termitomyces and indicate that specificity between termite hosts and fungi is not only visible from phylogenetic reconstructions, but also reflected physiologically. Differences between fungal symbionts of different hosts could facilitate fungal colonisation of the substrate collected by specific termites in incipient nests, potentially contributing to maintaining termite-fungus interaction specificity if physiological differences cause variable growth during competition between multiple fungal strains at the onset of fungus garden formation.

\section{Data accessibility}

Termitomyces ITS sequences have been deposited in GenBank.

\section{Acknowledgements}

We thank Z. Wilhelm de Beer, Michael J. Wingfield, and the staff and students at the Forestry and Agricultural Biotechnology Institute, University of Pretoria, for hosting field work; Christine Beemelmanns, René Benndorf, Saria Otani, Benjamin H. Conlon, Jane de Verges, Nick Bos, and Jeremy M. Thomas-Poulsen for help with excavations; Mandy Runderkamp, Margo Wisselink, and Kasun Bodawatta for laboratory assistance; and Ben Auxier for critically reading of the manuscript. This study was funded by the CAPES Foundation, Ministry of Education of Brazil (grant BEX: 13240/13-7) to RRdC, The Netherlands Organisation for Scientific Research Grant (ALW open competition 824.01.002) to SMEV, a Marie Curie International Incoming Fellowship (327940) to JZS, and a Villum Kann Rasmussen Young Investigator Fellowship (VKR10101) to MP. 


\section{Supplementary material}

Supplementary Table 1| Termitomyces basic medium - Schizophyllum commune minimal medium modified from Dons et al. (1979) with addition of urea.

\begin{tabular}{ll}
\hline Before autoclaving & Amount \\
\hline MgSO4*7H2O & $0.5 \mathrm{~g} / 1$ \\
\hline L-asparagine (monohydrate) & $1.5 \mathrm{~g} / 1$ \\
\hline Solution B & $1 \mathrm{ml} / 1$ \\
\hline Solution D & $2.5 \mathrm{ml} / 1$ \\
\hline Select agar & $15 \mathrm{~g} / 1$ \\
\hline After autoclaving & \\
\hline Solution A & $1 \mathrm{ml} / 1$ \\
\hline Solution C & $1 \mathrm{ml} / 1$ \\
\hline Urea solution & $5 \mathrm{ml} / 1$ \\
\hline Stock solutions & \\
\hline Solution A (filter sterilised): & \\
\hline Thiaminiumdichloride & $0.12 \mathrm{~g} / 1$ \\
\hline Solution B (filter sterilised): & \\
\hline FeCl3*6H2O & $5 \mathrm{~g} / 1$ \\
\hline Solution C (filter sterilised): & \\
\hline HBO3 & $0.06 \mathrm{~g} / 1$ \\
\hline (NH4)Mo7O24*4H2O & $0.04 \mathrm{~g} / 1$ \\
\hline CuSO4*5H2O & $0.2 \mathrm{~g} / 1$ \\
\hline ZnSO4*7H2O & $2.0 \mathrm{~g} / 1$ \\
\hline MnSO4*4H2O & $0.1 \mathrm{~g} / 1$ \\
\hline CoCl2*6H2O & $0.4 \mathrm{~g} / 1$ \\
\hline Ca(NO3)2*4H2O & $1.2 \mathrm{~g} / 1$ \\
\hline Solution D (filter sterilised): & \\
\hline KH2PO4 (water free) & $184 \mathrm{~g} / 1$ \\
\hline K2HPO4 (water free) & $400 \mathrm{~g} / 1$ \\
\hline Urea Solution (filter sterilised): & \\
\hline Urea & \\
\hline
\end{tabular}


Supplementary Table $2 \mid$ Days of incubation on different carbon sources for each fungal isolate before biomass collection and photographing.

\begin{tabular}{|llll}
\hline Termite species & Strain & $\begin{array}{l}\text { Days of incubation for } \\
\text { biomass harvesting }\end{array}$ & $\begin{array}{l}\text { Days incubated } \\
\text { for photo }\end{array}$ \\
\hline Macrotermes natalensis & Mn156 & 23 & 23 \\
& Mn160 & 26 & 22 \\
& Mn162 & 26 & 23 \\
\hline Odontotermes sp. & Od127 & 22 & 24 \\
& Od128 & 31 & 24 \\
\hline Odontotermes cf. badius & Od159 & 21 & 28 \\
& Od150 & 22 & 28 \\
& Od151 & 21 & 26 \\
\hline
\end{tabular}

Supplementary Table $3 \mid$ Contribution (loading values) of performance on individual carbon sources to the separation of different Termitomyces isolates in PCA space (Figure 2).

\begin{tabular}{|c|c|c|c|c|c|c|}
\hline & $\begin{array}{l}\text { Variable (carbon } \\
\text { sources) }\end{array}$ & $\begin{array}{l}\text { Dimen- } \\
\text { sion } 1\end{array}$ & $\begin{array}{l}\text { Dimen- } \\
\text { sion } 2\end{array}$ & $\begin{array}{l}\text { Dimen- } \\
\text { sion } 3\end{array}$ & $\begin{array}{l}\text { Dimen- } \\
\text { sion } 4\end{array}$ & $\begin{array}{l}\text { Dimen- } \\
\text { sion } 5\end{array}$ \\
\hline \multirow{7}{*}{$\frac{\frac{x}{0}}{\stackrel{a}{a}}$} & Wheat bran & -0.156 & 0.240 & 0.089 & 0.009 & -0.218 \\
\hline & Sugar beet pulp & -0.125 & 0.064 & 0.204 & 0.020 & 0.475 \\
\hline & Citrus pulp & -0.152 & 0.180 & -0.254 & 0.064 & -0.025 \\
\hline & Soybean hulls & -0.178 & 0.236 & 0.028 & -0.106 & -0.020 \\
\hline & Rice bran & 0.176 & 0.138 & 0.092 & 0.283 & 0.133 \\
\hline & Cotton seed pulp & -0.209 & 0.160 & 0.041 & 0.112 & 0.025 \\
\hline & Alfalfa meal & -0.204 & 0.197 & 0.028 & 0.109 & -0.057 \\
\hline \multirow{11}{*}{ 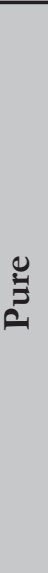 } & Guar gum & -0.208 & 0.200 & 0.080 & 0.014 & -0.065 \\
\hline & Arabic gum & 0.217 & 0.155 & -0.020 & 0.128 & 0.199 \\
\hline & Apple pectin & 0.139 & 0.136 & -0.175 & 0.389 & -0.060 \\
\hline & Citrus pectin & -0.091 & 0.273 & -0.067 & 0.182 & 0.208 \\
\hline & Arabino galactan & -0.186 & 0.198 & 0.080 & -0.101 & 0.042 \\
\hline & Chitin & -0.130 & 0.261 & -0.006 & -0.089 & -0.126 \\
\hline & Lignin & -0.105 & 0.275 & 0.087 & -0.009 & 0.196 \\
\hline & Cellulose & 0.211 & 0.116 & -0.219 & -0.017 & -0.067 \\
\hline & Soluble starch & 0.092 & 0.260 & -0.137 & 0.164 & 0.121 \\
\hline & Inulin & 0.151 & 0.189 & -0.099 & 0.172 & 0.043 \\
\hline & Beechwood xylan & 0.166 & -0.137 & 0.225 & 0.010 & 0.182 \\
\hline
\end{tabular}


Supplementary Table 3 continued | Contribution (loading values) of performance on individual carbon sources to the separation of different Termitomyces isolates in PCA space (Figure 2).

\begin{tabular}{|c|c|c|c|c|c|c|}
\hline & $\begin{array}{l}\text { Variable (carbon } \\
\text { sources) }\end{array}$ & $\begin{array}{l}\text { Dimen- } \\
\text { sion } 1\end{array}$ & $\begin{array}{l}\text { Dimen- } \\
\text { sion } 2\end{array}$ & $\begin{array}{l}\text { Dimen- } \\
\text { sion } 3\end{array}$ & $\begin{array}{l}\text { Dimen- } \\
\text { sion } 4\end{array}$ & $\begin{array}{l}\text { Dimen- } \\
\text { sion } 5\end{array}$ \\
\hline \multirow{5}{*}{ 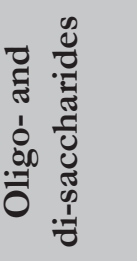 } & Cellobiose & -0.088 & 0.112 & 0.354 & 0.031 & -0.233 \\
\hline & Maltose & 0.118 & 0.177 & 0.012 & -0.283 & 0.226 \\
\hline & Lactose & -0.131 & 0.031 & 0.354 & -0.045 & 0.052 \\
\hline & Sucrose & 0.189 & 0.150 & 0.212 & -0.128 & 0.062 \\
\hline & Raffinose & -0.111 & -0.037 & 0.006 & 0.236 & -0.153 \\
\hline \multirow{10}{*}{ 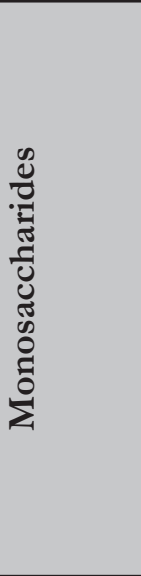 } & D-glucose & 0.239 & 0.032 & 0.159 & 0.116 & -0.045 \\
\hline & D-fructose & 0.191 & 0.064 & 0.136 & 0.344 & 0.015 \\
\hline & D-galactose & 0.176 & 0.181 & -0.028 & -0.257 & -0.190 \\
\hline & D-mannose & 0.069 & -0.017 & 0.389 & 0.125 & -0.177 \\
\hline & D-ribose & 0.169 & 0.206 & 0.019 & -0.278 & 0.038 \\
\hline & D-xylose & 0.106 & 0.050 & 0.367 & 0.096 & -0.191 \\
\hline & L-arabinose & 0.238 & 0.014 & -0.145 & -0.021 & -0.185 \\
\hline & L-rhamnose & 0.174 & 0.234 & -0.075 & -0.042 & 0.108 \\
\hline & $\begin{array}{l}\text { D-galacturonic } \\
\text { acid }\end{array}$ & 0.245 & 0.019 & 0.089 & 0.105 & -0.187 \\
\hline & D-glucuronic acid & 0.042 & -0.221 & 0.123 & 0.029 & 0.416 \\
\hline Protein & Casein & 0.233 & 0.134 & 0.078 & -0.121 & -0.034 \\
\hline Control & No carbon source & 0.200 & 0.110 & 0.068 & -0.339 & -0.019 \\
\hline
\end{tabular}

Supplementary Table 4| Principal component analysis Eigen values follow by their cumulative percentage of variance (Figure 2).

\begin{tabular}{lll}
\hline $\begin{array}{l}\text { Principle } \\
\text { Component }\end{array}$ & Eigenvalue & $\begin{array}{l}\text { Cumulative \% } \\
\text { of variance }\end{array}$ \\
\hline PC1 & 13.78 & 39.38 \\
\hline PC2 & 8.117 & 62.57 \\
PC3 & 4.951 & 76.72 \\
\hline PC4 & 2.474 & 84.57 \\
PC5 & 2.159 & 90.74 \\
PC6 & 1.604 & 95.32 \\
PC7 & 0.906 & 97.91 \\
PC8 & 0.728 & 100 \\
\hline
\end{tabular}


Supplementary Table 5 $\mid$ Complete media recipe for 36 artificial diets using different ratios (9:1 to $1: 9)$ and concentrations ( 8 to $80 \mathrm{~g} / \mathrm{L}$ ) of Protein:Carbohydrates (modified from Shik et al. 2016). All diets were prepared in $1000 \mathrm{~mL}$ of distilled water and all amounts are in grams. Ingredient amounts adjusted relative to the mass of protein and carbohydrates they contained.

\begin{tabular}{|c|c|c|c|c|c|c|c|}
\hline P:C & $\begin{array}{l}\text { Bacto- } \\
\text { peptone }\end{array}$ & $\begin{array}{l}\text { Trypticase } \\
\text { peptone }\end{array}$ & $\begin{array}{l}\text { Bacto } \\
\text { tryptone }\end{array}$ & Glucose & Starch & Vitamin & Agar \\
\hline \multicolumn{8}{|c|}{ Diet concentration: $8 \mathrm{~g} / \mathrm{L}$} \\
\hline $9: 1$ & 2.585 & 2.554 & 2.555 & 0.381 & 0.381 & 0.160 & 16.00 \\
\hline $6: 1$ & 2.461 & 2.431 & 2.433 & 0.555 & 0.555 & 0.160 & 16.00 \\
\hline $3: 1$ & 2.128 & 2.129 & 2.154 & 0.984 & 0.984 & 0.160 & 16.00 \\
\hline $2: 1$ & 1.895 & 1.896 & 1.918 & 1.315 & 1.315 & 0.160 & 16.00 \\
\hline $1: 1$ & 1.419 & 1.420 & 1.436 & 1.990 & 1.990 & 0.160 & 16.00 \\
\hline $1: 2$ & 0.943 & 0.943 & 0.954 & 2.664 & 2.664 & 0.160 & 16.00 \\
\hline $1: 3$ & 0.709 & 0.710 & 0.718 & 2.995 & 2.995 & 0.160 & 16.00 \\
\hline 1:6 & 0.406 & 0.406 & 0.411 & 3.425 & 3.425 & 0.160 & 16.00 \\
\hline 1:9 & 0.284 & 0.284 & 0.287 & 3.598 & 3.598 & 0.160 & 16.00 \\
\hline \multicolumn{8}{|c|}{ Diet concentration: $32 \mathrm{~g} / \mathrm{L}$} \\
\hline $9: 1$ & 10.21 & 10.22 & 10.34 & 1.525 & 1.525 & 0.640 & 16.00 \\
\hline $6: 1$ & 9.725 & 9.731 & 9.845 & 2.219 & 2.219 & 0.640 & 16.00 \\
\hline $3: 1$ & 8.512 & 8.517 & 8.617 & 3.938 & 3.938 & 0.640 & 16.00 \\
\hline $2: 1$ & 7.579 & 7.583 & 7.672 & 5.261 & 5.261 & 0.640 & 16.00 \\
\hline $1: 1$ & 5.675 & 5.678 & 5.745 & 7.958 & 7.958 & 0.640 & 16.00 \\
\hline $1: 2$ & 3.771 & 3.773 & 3.817 & 10.65 & 10.65 & 0.640 & 16.00 \\
\hline $1: 3$ & 2.837 & 2.839 & 2.872 & 11.97 & 11.97 & 0.640 & 16.00 \\
\hline $1: 6$ & 1.624 & 1.625 & 1.644 & 13.69 & 13.69 & 0.640 & 16.00 \\
\hline 1:9 & 1.135 & 1.136 & 1.149 & 14.39 & 14.39 & 0.640 & 16.00 \\
\hline \multicolumn{8}{|c|}{ Diet concentration: $56 \mathrm{~g} / \mathrm{L}$} \\
\hline $9: 1$ & 17.88 & 17.89 & 18.10 & 2.669 & 2.669 & 1.120 & 16.00 \\
\hline $6: 1$ & 17.02 & 17.03 & 17.23 & 3.883 & 3.883 & 1.120 & 16.00 \\
\hline $3: 1$ & 14.90 & 14.91 & 15.08 & 6.891 & 6.891 & 1.120 & 16.00 \\
\hline $2: 1$ & 13.26 & 13.27 & 13.43 & 9.206 & 9.206 & 1.120 & 16.00 \\
\hline $1: 1$ & 9.931 & 9.937 & 10.05 & 13.93 & 13.93 & 1.120 & 16.00 \\
\hline $1: 2$ & 6.599 & 6.603 & 6.681 & 18.65 & 18.65 & 1.120 & 16.00 \\
\hline $1: 3$ & 4.965 & 4.968 & 5.027 & 20.96 & 20.96 & 1.120 & 16.00 \\
\hline $1: 6$ & 2.843 & 2.844 & 2.878 & 23.97 & 23.97 & 1.120 & 16.00 \\
\hline 1:9 & 1.986 & 1.987 & 2.011 & 25.19 & 25.19 & 1.120 & 16.00 \\
\hline
\end{tabular}


Supplementary Table $\mathbf{5}$ continued $\mid$ Complete media recipe for 36 artificial diets using different ratios (9:1 to 1:9) and concentrations (8 to $80 \mathrm{~g} / \mathrm{L}$ ) of Protein:Carbohydrates (modified from Shik et al. 2016). All diets were prepared in $1000 \mathrm{~mL}$ of distilled water and all amounts are in grams. Ingredient amounts adjusted relative to the mass of protein and carbohydrates they contained.

\begin{tabular}{llllllll}
\hline P:C & $\begin{array}{c}\text { Bacto- } \\
\text { peptone }\end{array}$ & $\begin{array}{c}\text { Trypticase } \\
\text { peptone }\end{array}$ & $\begin{array}{l}\text { Bacto } \\
\text { tryptone }\end{array}$ & Glucose & Starch & Vitamin & Agar \\
\hline \multicolumn{7}{l}{ Diet concentration: $80 \mathrm{~g} / \mathrm{L}$} \\
\hline $9: 1$ & 25.54 & 25.55 & 25.85 & 3.813 & 3.813 & 1.600 & 16.00 \\
\hline $6: 1$ & 24.31 & 24.33 & 24.61 & 5.547 & 5.547 & 1.600 & 16.00 \\
\hline $3: 1$ & 21.28 & 21.29 & 21.54 & 9.845 & 9.845 & 1.600 & 16.00 \\
\hline $2: 1$ & 18.95 & 18.96 & 19.18 & 13.15 & 13.15 & 1.600 & 16.00 \\
$1: 1$ & 14.19 & 14.20 & 14.36 & 19.90 & 19.90 & 1.600 & 16.00 \\
$1: 2$ & 9.427 & 9.433 & 9.544 & 26.64 & 26.64 & 1.600 & 16.00 \\
$1: 3$ & 7.093 & 7.098 & 7.181 & 29.95 & 29.95 & 1.600 & 16.00 \\
$1: 6$ & 4.061 & 4.063 & 4.111 & 34.25 & 34.25 & 1.600 & 16.00 \\
$1: 9$ & 2.837 & 2.839 & 2.872 & 35.98 & 35.98 & 1.600 & 16.00 \\
\hline
\end{tabular}

Supplementary Table 6 $\mid$ Loading values of performance in different growth conditions (i.e., ratios and concentrations of P:C) to the separation of Termitomyces isolates in PCA space (Figure 3).

\begin{tabular}{|llllll}
\hline $\begin{array}{l}\text { Variable (Concentra- } \\
\text { tion and P:C ratio) }\end{array}$ & $\begin{array}{l}\text { Dimen- } \\
\text { sion 1 }\end{array}$ & $\begin{array}{l}\text { Dimen- } \\
\text { sion 2 }\end{array}$ & $\begin{array}{l}\text { Dimen- } \\
\text { sion 3 }\end{array}$ & $\begin{array}{l}\text { Dimen- } \\
\text { sion 4 }\end{array}$ & $\begin{array}{l}\text { Dimen- } \\
\text { sion 5 }\end{array}$ \\
\hline 8 g/L 1:9 & 0.125 & 0.283 & 0.157 & -0.093 & 0.015 \\
\hline 8 g/L 1:6 & -0.034 & 0.032 & 0.310 & -0.160 & -0.242 \\
\hline 8 g/L 1:3 & 0.216 & -0.184 & 0.036 & 0.115 & 0.156 \\
\hline 8 g/L 1:2 & 0.075 & -0.168 & 0.150 & 0.346 & 0.095 \\
\hline 8 g/L 1:1 & -0.012 & 0.279 & -0.212 & 0.042 & -0.057 \\
\hline 8 g/L 2:1 & -0.055 & 0.262 & -0.069 & 0.169 & 0.180 \\
\hline 8 g/L 3:1 & -0.191 & 0.253 & 0.004 & 0.037 & -0.121 \\
\hline 8 g/L 6:1 & -0.229 & 0.079 & -0.011 & 0.204 & 0.130 \\
\hline 8 g/L 9:1 & 0.055 & -0.183 & -0.254 & 0.065 & -0.278 \\
\hline 32 g/L 1:9 & 0.172 & 0.175 & 0.168 & -0.133 & -0.200 \\
\hline 32 g/L 1:6 & 0.145 & 0.134 & 0.245 & -0.154 & -0.010 \\
\hline 32 g/L 1:3 & 0.187 & 0.237 & 0.071 & 0.119 & -0.027 \\
\hline 32 g/L 1:2 & 0.212 & 0.181 & 0.109 & 0.107 & -0.039 \\
\hline 32 g/L 1:1 & 0.242 & -0.002 & -0.144 & -0.048 & 0.194 \\
\hline 32 g/L 2:1 & 0.243 & -0.043 & -0.124 & 0.059 & 0.183 \\
\hline
\end{tabular}




\begin{tabular}{|c|c|c|c|c|c|}
\hline $\begin{array}{l}\text { Variable (Concentra- } \\
\text { tion and P:C ratio) }\end{array}$ & $\begin{array}{l}\text { Dimen- } \\
\text { sion } 1\end{array}$ & $\begin{array}{l}\text { Dimen- } \\
\text { sion } 2\end{array}$ & $\begin{array}{l}\text { Dimen- } \\
\text { sion } 3\end{array}$ & $\begin{array}{l}\text { Dimen- } \\
\text { sion } 4\end{array}$ & $\begin{array}{l}\text { Dimen- } \\
\text { sion } 5\end{array}$ \\
\hline $32 \mathrm{~g} / \mathrm{L} 3: 1$ & 0.254 & 0.050 & 0.056 & 0.057 & 0.160 \\
\hline 32 g/L 6:1 & 0.126 & 0.152 & -0.110 & 0.306 & 0.125 \\
\hline 32 g/L 9:1 & 0.009 & -0.189 & -0.278 & 0.017 & -0.256 \\
\hline $56 \mathrm{~g} / \mathrm{L} 1: 9$ & 0.162 & 0.198 & 0.241 & -0.113 & -0.047 \\
\hline $56 \mathrm{~g} / \mathrm{L} 1: 6$ & -0.180 & 0.022 & 0.155 & -0.113 & 0.377 \\
\hline $56 \mathrm{~g} / \mathrm{L} 1: 3$ & -0.206 & 0.166 & -0.023 & -0.210 & 0.095 \\
\hline $56 \mathrm{~g} / \mathrm{L} 1: 2$ & 0.102 & 0.147 & -0.175 & 0.173 & 0.054 \\
\hline $56 \mathrm{~g} / \mathrm{L} 1: 1$ & -0.128 & -0.108 & 0.283 & 0.206 & -0.136 \\
\hline $56 \mathrm{~g} / \mathrm{L} 2: 1$ & 0.052 & 0.098 & -0.059 & -0.386 & 0.137 \\
\hline $56 \mathrm{~g} / \mathrm{L} 3: 1$ & 0.162 & 0.104 & 0.097 & 0.203 & 0.023 \\
\hline 56 g/L 6:1 & -0.025 & 0.238 & 0.136 & 0.240 & 0.173 \\
\hline $56 \mathrm{~g} / \mathrm{L} 9: 1$ & 0.191 & -0.131 & -0.184 & 0.074 & 0.157 \\
\hline 80 g/L 1:9 & 0.218 & -0.024 & -0.179 & -0.146 & -0.083 \\
\hline 80 g/L 1:6 & 0.248 & 0.022 & -0.068 & -0.130 & 0.075 \\
\hline 80 g/L 1:3 & 0.247 & 0.065 & -0.117 & -0.095 & -0.026 \\
\hline $80 \mathrm{~g} / \mathrm{L} 1: 2$ & 0.204 & 0.027 & -0.087 & -0.286 & 0.026 \\
\hline $80 \mathrm{~g} / \mathrm{L} 1: 1$ & 0.129 & 0.188 & -0.013 & 0.142 & -0.416 \\
\hline $80 \mathrm{~g} / \mathrm{L} \mathrm{2:1}$ & 0.188 & -0.063 & 0.130 & 0.181 & -0.297 \\
\hline 80 g/L 3:1 & 0.076 & -0.241 & 0.256 & -0.087 & 0.012 \\
\hline $80 \mathrm{~g} / \mathrm{L} 6: 1$ & 0.136 & -0.230 & 0.250 & 0.007 & 0.091 \\
\hline $80 \mathrm{~g} / \mathrm{L}$ 9:1 & 0.159 & -0.233 & 0.214 & 0.008 & 0.103 \\
\hline
\end{tabular}

Supplementary Table $7 \mid$ Principal component analysis Eigen values follow by their cumulative variance (Figure 3).

\begin{tabular}{lll}
\hline $\begin{array}{l}\text { Principle } \\
\text { Component }\end{array}$ & Eigenvalue & $\begin{array}{l}\text { Cumulative \% } \\
\text { of variance }\end{array}$ \\
\hline PC1 & 12.9430855 & 36,0 \\
\hline PC2 & 7.2119164 & 55.0 \\
PC3 & 5.2983926 & 70.7 \\
PC4 & 4.7632778 & 83.9 \\
\hline PC5 & 2.4075263 & 90.6 \\
PC6 & 1.4994803 & 94.8 \\
\hline PC7 & 1.3576969 & 98.6 \\
\hline PC8 & 0.5186243 & 100
\end{tabular}


Supplementary Table 8| GLM analyses comparing growth responses (area and biomass) at the termite species level, across Termitomyces isolates isolated from Odontotermes sp. (Od127, Od128, Od159), Odontotermes cf. badius (Od145, Od150, Od150), and Macrotermes natalensis (Mn156, Mn160, Mn162). Since none of the treatment by termite colony interactions were significant, we calculated averages across colonies within termite species when preparing Figure 3 in the manuscript.

\begin{tabular}{|c|c|c|c|c|c|c|}
\hline $\begin{array}{l}\text { Termite } \\
\text { species }\end{array}$ & $\begin{array}{l}\text { Depen- } \\
\text { dent } \\
\text { variable }\end{array}$ & Factor & $\begin{array}{l}\text { Degrees } \\
\text { of free- } \\
\text { dom }\end{array}$ & $\begin{array}{l}\text { Type III } \\
\text { Mean } \\
\text { square }\end{array}$ & F value & P value \\
\hline \multirow{22}{*}{ 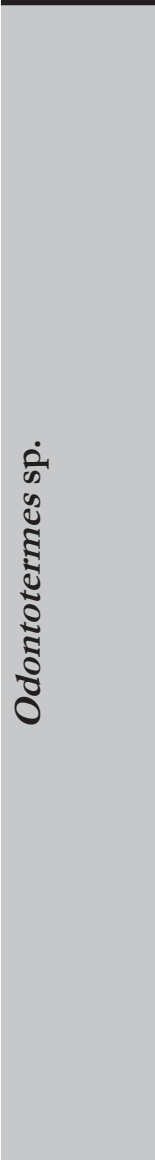 } & \multirow{11}{*}{ 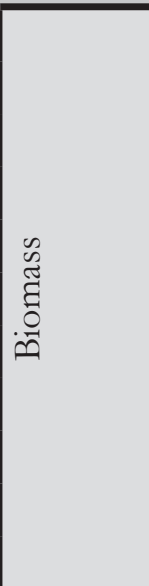 } & $\mathrm{P}$ & 1 & 0.004 & 0.30 & 0.5854 \\
\hline & & C & 1 & 3.790 & 275.7 & $<.0001$ \\
\hline & & colony & 2 & 0.002 & 0.13 & 0.8773 \\
\hline & & $\mathrm{P} \times \mathrm{P}$ & 1 & 0.096 & 6.69 & 0.0088 \\
\hline & & $\mathrm{C} \times \mathrm{C}$ & 1 & 4.267 & 310.4 & $<.0001$ \\
\hline & & $\mathrm{P} \times \mathrm{C}$ & 1 & 0.208 & 15.10 & 0.0001 \\
\hline & & P x colony & 2 & 0.006 & 0.41 & 0.6654 \\
\hline & & C x colony & 2 & 0.007 & 0.48 & 0.6177 \\
\hline & & $\mathrm{P} \times \mathrm{P} \times$ colony & 2 & 0.003 & 0.21 & 0.8096 \\
\hline & & C x C x colony & 2 & 0.011 & 0.81 & 0.4447 \\
\hline & & P x C x colony & 2 & 0.009 & 0.72 & 0.4856 \\
\hline & \multirow{11}{*}{ 导 } & $\mathrm{P}$ & 1 & 22.65 & 7.68 & 0.0059 \\
\hline & & C & 1 & 61.15 & 20.72 & $<.0001$ \\
\hline & & colony & 2 & 3.437 & 1.16 & 0.3134 \\
\hline & & $\mathrm{P} \times \mathrm{P}$ & 1 & 144.1 & 45.85 & $<.0001$ \\
\hline & & $\mathrm{C} \times \mathrm{C}$ & 1 & 7.153 & 2.42 & 0.1206 \\
\hline & & $\mathrm{P} \times \mathrm{C}$ & 1 & 0.904 & 0.31 & 0.5802 \\
\hline & & P x colony & 2 & 1.085 & 0.37 & 0.6925 \\
\hline & & C x colony & 2 & 2.993 & 1.01 & 0.3640 \\
\hline & & P x P x colony & 2 & 0.605 & 0.21 & 0.8145 \\
\hline & & $\mathrm{C} \times \mathrm{C}$ x colony & 2 & 2.246 & 0.76 & 0.4680 \\
\hline & & $\mathrm{P} \times \mathrm{C} \times$ colony & 2 & 1.329 & 0.45 & 0.6378 \\
\hline
\end{tabular}


Supplementary Table 8 continued| GLM analyses comparing growth responses (area and biomass) at the termite species level, across Termitomyces isolates isolated from Odontotermes sp. (Od127, Od128, Od159), Odontotermes cf. badius (Od145, Od150, Od150), and Macrotermes natalensis (Mn156, Mn160, Mn162). Since none of the treatment by termite colony interactions were significant, we calculated averages across colonies within termite species when preparing Figure 3 in the manuscript.

\begin{tabular}{|c|c|c|c|c|c|c|}
\hline $\begin{array}{l}\text { Termite } \\
\text { species }\end{array}$ & $\begin{array}{l}\text { Depen- } \\
\text { dent } \\
\text { variable }\end{array}$ & Factor & $\begin{array}{l}\text { Degrees } \\
\text { of free- } \\
\text { dom }\end{array}$ & $\begin{array}{l}\text { Type III } \\
\text { Mean } \\
\text { square }\end{array}$ & $F$ value & $P$ value \\
\hline \multirow{22}{*}{ 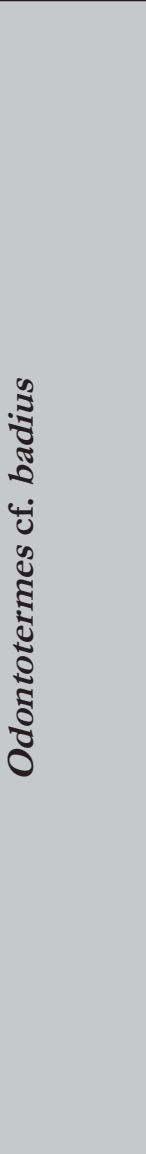 } & \multirow{11}{*}{ 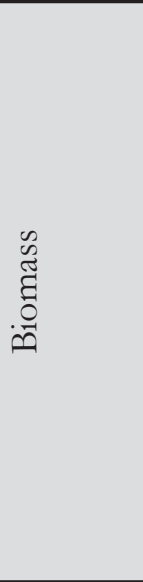 } & $\mathrm{P}$ & 1 & 0.082 & 4.14 & 0.042 \\
\hline & & $\mathrm{C}$ & 1 & 4.291 & 214.0 & $<.0001$ \\
\hline & & colony & 2 & 0.017 & 0.86 & 0.4225 \\
\hline & & $\mathrm{P} \times \mathrm{P}$ & 1 & 0.008 & 0.44 & 0.5071 \\
\hline & & $\mathrm{C} \times \mathrm{C}$ & 1 & 4.790 & 238.9 & $<.0001$ \\
\hline & & $\mathrm{P} \times \mathrm{C}$ & 1 & 0.106 & 5.30 & 0.0220 \\
\hline & & P x colony & 2 & 0.003 & 0.18 & 0.8338 \\
\hline & & C x colony & 2 & 0.015 & 0.78 & 0.4581 \\
\hline & & $\mathrm{P} \times \mathrm{P} \times$ colony & 2 & 0.002 & 0.13 & 0.8754 \\
\hline & & $\mathrm{C} \times \mathrm{C} x$ colony & 2 & 0.010 & 0.55 & 0.5798 \\
\hline & & $\mathrm{P} \times \mathrm{C}$ x colony & 2 & 0.004 & 0.21 & 0.8146 \\
\hline & \multirow{11}{*}{ 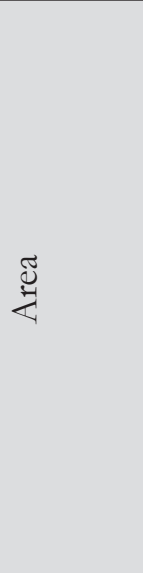 } & $\mathrm{P}$ & 1 & 1.774 & 1.10 & 0.2955 \\
\hline & & $\mathrm{C}$ & 1 & 2.046 & 1.27 & 0.2613 \\
\hline & & colony & 2 & 0.800 & 0.50 & 0.6098 \\
\hline & & $\mathrm{P} \times \mathrm{P}$ & 1 & 39.32 & 24.34 & $<.0001$ \\
\hline & & $\mathrm{C} \times \mathrm{C}$ & 1 & 36.59 & 22.65 & $<.0001$ \\
\hline & & $\mathrm{P} \times \mathrm{C}$ & 1 & 0.484 & 0.30 & 0.5846 \\
\hline & & $\mathrm{P} \times$ colony & 2 & 0.906 & 0.56 & 0.5712 \\
\hline & & C x colony & 2 & 4.199 & 2.60 & 0.0760 \\
\hline & & $\mathrm{P} \times \mathrm{P} \times$ colony & 2 & 0.597 & 0.37 & 0.6910 \\
\hline & & $\mathrm{C} \times \mathrm{C}$ x colony & 2 & 6.147 & 3.80 & 0.0233 \\
\hline & & $\mathrm{P} \times \mathrm{C} \times$ colony & 2 & 0.296 & 0.18 & 0.8325 \\
\hline
\end{tabular}


Supplementary Table 8 continued| GLM analyses comparing growth responses (area and biomass) at the termite species level, across Termitomyces isolates isolated from Odontotermes sp. (Od127, Od128, Od159), Odontotermes cf. badius (Od145, Od150, Od150), and Macrotermes natalensis (Mn156, Mn160, Mn162). Since none of the treatment by termite colony interactions were significant, we calculated averages across colonies within termite species when preparing Figure 3 in the manuscript.

\begin{tabular}{|c|c|c|c|c|c|c|}
\hline $\begin{array}{l}\text { Termite } \\
\text { species }\end{array}$ & $\begin{array}{l}\text { Depen- } \\
\text { dent } \\
\text { variable }\end{array}$ & Factor & $\begin{array}{l}\text { Degrees } \\
\text { of free- } \\
\text { dom }\end{array}$ & $\begin{array}{l}\text { Type III } \\
\text { Mean } \\
\text { square }\end{array}$ & $F$ value & $P$ value \\
\hline \multirow{22}{*}{ 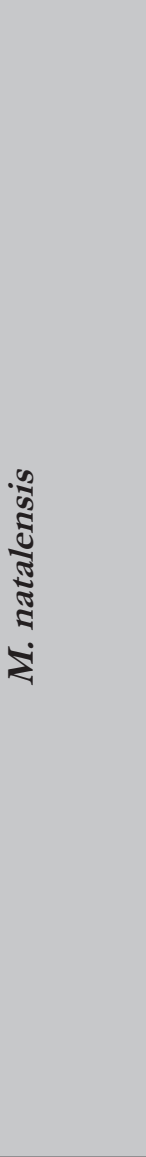 } & \multirow{11}{*}{ 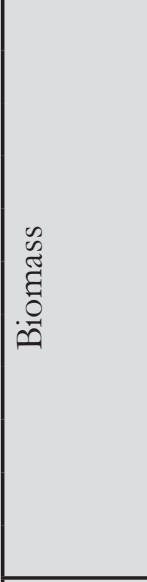 } & P & 1 & 0.102 & 5.01 & 0.0260 \\
\hline & & $\mathrm{C}$ & 1 & 3.010 & 147.6 & $<.0001$ \\
\hline & & colony & 2 & 0.001 & 0.10 & 0.9084 \\
\hline & & $\mathrm{P} \times \mathrm{P}$ & 1 & 0.002 & 0.12 & 0.7296 \\
\hline & & $\mathrm{C} \times \mathrm{C}$ & 1 & 3.587 & 175.8 & $<.0001$ \\
\hline & & $\mathrm{P} \times \mathrm{C}$ & 1 & 0.046 & 2.26 & 0.1340 \\
\hline & & $\mathrm{P} \times$ colony & 2 & 0.050 & 2.48 & 0.0850 \\
\hline & & C x colony & 2 & 0.060 & 2.94 & 0.0542 \\
\hline & & $\mathrm{P} \times \mathrm{P} \times$ colony & 2 & 0.069 & 3.43 & 0.0338 \\
\hline & & $\mathrm{C} \times \mathrm{C} x$ colony & 2 & 0.068 & 3.36 & 0.0362 \\
\hline & & $\mathrm{P} \times \mathrm{C}$ x colony & 2 & 0.004 & 0.22 & 0.8055 \\
\hline & \multirow{11}{*}{ 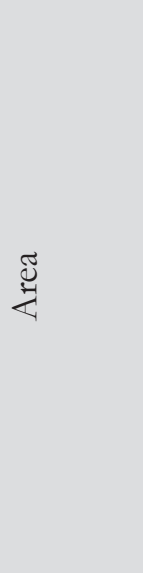 } & $\mathrm{P}$ & 1 & 14.66 & 7.26 & 0.0075 \\
\hline & & $\mathrm{C}$ & 1 & 21.82 & 10.80 & 0.0011 \\
\hline & & colony & 2 & 0.745 & 0.37 & 0.6920 \\
\hline & & $\mathrm{P} \times \mathrm{P}$ & 1 & 61.20 & 30.28 & $<.0001$ \\
\hline & & $\mathrm{C} \times \mathrm{C}$ & 1 & 68.09 & 33.69 & $<.0001$ \\
\hline & & $\mathrm{P} \times \mathrm{C}$ & 1 & 52.15 & 25.80 & $<.0001$ \\
\hline & & P x colony & 2 & 0.432 & 0.21 & 0.8076 \\
\hline & & C x colony & 2 & 0.382 & 0.19 & 0.8277 \\
\hline & & $\mathrm{P} \times \mathrm{P} \times$ colony & 2 & 0.217 & 0.11 & 0.8978 \\
\hline & & $\mathrm{C} \times \mathrm{C}$ x colony & 2 & 0.134 & 0.07 & 0.9359 \\
\hline & & $\mathrm{P} \times \mathrm{C} \times$ colony & 2 & 0.330 & 0.16 & 0.8491 \\
\hline
\end{tabular}


Supplementary Table 9| Univariate tests of parameter estimates of two opposing measures of fungal growth in three fungus-growing termite species: (i) production of fungal biomass (gram after 20 days of incubation), and (ii) radial growth ( $\mathrm{cm} 2$ after 24 days of incubation) across a nutritional landscape of 36 diets varying across nine P:C ratios (between 1:9 and 9:1) and four protein and carbohydrate concentrations $(8,32,56$, and $80 \mathrm{~g} / \mathrm{L})$. Results are from least-square regressions, estimating nonlinear parametric response surfaces with the linear and quadratic components of protein $(\mathrm{P})$ and carbohydrate $(\mathrm{C})$ composition in the diets, and the $\mathrm{P}$ $\times \mathrm{C}$ interaction.

\begin{tabular}{|c|c|c|c|c|c|}
\hline $\begin{array}{l}\text { Termite } \\
\text { species }\end{array}$ & $\begin{array}{l}\text { Dependent } \\
\text { variable }\end{array}$ & Parameter & $\begin{array}{l}\text { Degrees of } \\
\text { freedom }\end{array}$ & F value & $P$ value \\
\hline \multirow{10}{*}{ 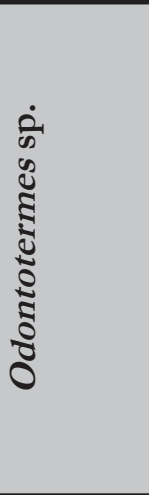 } & \multirow{5}{*}{ 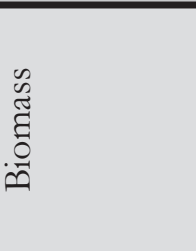 } & $\mathrm{P}$ & 1 & 165.6 & $<.0001$ \\
\hline & & $\mathrm{C}$ & 1 & 11.76 & 0.0007 \\
\hline & & P2 & 1 & 15.80 & $<.0001$ \\
\hline & & C2 & 1 & 296.6 & $<.0001$ \\
\hline & & $\mathrm{PxC}$ & 1 & 14.93 & 0.0001 \\
\hline & \multirow{5}{*}{$\stackrel{\Xi}{\dot{Z}}$} & $\mathrm{P}$ & 1 & 130.6 & $<.0001$ \\
\hline & & $\mathrm{C}$ & 1 & 125.5 & $<.0001$ \\
\hline & & P2 & 1 & 52.27 & $<.0001$ \\
\hline & & $\mathrm{C} 2$ & 1 & 2.11 & 0.1472 \\
\hline & & $\mathrm{PxC}$ & 1 & 0.29 & 0.5921 \\
\hline \multirow{10}{*}{ 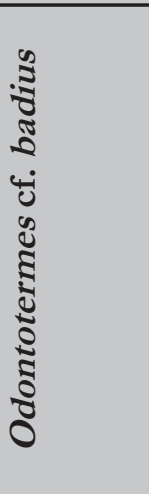 } & \multirow{5}{*}{ 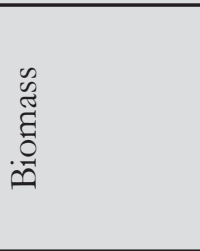 } & $\mathrm{P}$ & 1 & 4.14 & 0.0428 \\
\hline & & $\mathrm{C}$ & 1 & 214.0 & $<.0001$ \\
\hline & & P2 & 1 & 0.44 & 0.5071 \\
\hline & & C2 & 1 & 238.9 & $<.0001$ \\
\hline & & $\mathrm{PxC}$ & 1 & 5.30 & 0.0220 \\
\hline & \multirow{5}{*}{$\stackrel{Ð}{\sharp}$} & $\mathrm{P}$ & 1 & 98.13 & $<.0001$ \\
\hline & & $\mathrm{C}$ & 1 & 99.03 & $<.0001$ \\
\hline & & P2 & 1 & 35.67 & $<.0001$ \\
\hline & & C2 & 1 & 25.86 & $<.0001$ \\
\hline & & $\mathrm{PxC}$ & 1 & 0.36 & 0.5492 \\
\hline \multirow{10}{*}{ 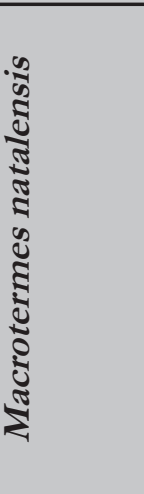 } & \multirow{5}{*}{ 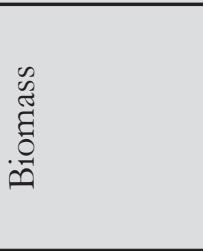 } & $\mathrm{P}$ & 1 & 5.01 & 0.0260 \\
\hline & & $\mathrm{C}$ & 1 & 147.6 & $<.0001$ \\
\hline & & P2 & 1 & 0.12 & 0.7296 \\
\hline & & $\mathrm{C} 2$ & 1 & 175.8 & $<.0001$ \\
\hline & & $\mathrm{PxC}$ & 1 & 2.26 & 0.1340 \\
\hline & \multirow{5}{*}{$\stackrel{\varpi}{\xi}$} & $\mathrm{P}$ & 1 & 7.26 & 0.0075 \\
\hline & & $\mathrm{C}$ & 1 & 10.80 & 0.0011 \\
\hline & & P2 & 1 & 30.28 & $<.0001$ \\
\hline & & $\mathrm{C} 2$ & 1 & 33.69 & $<.0001$ \\
\hline & & $\mathrm{PxC}$ & 1 & 25.80 & $<.0001$ \\
\hline
\end{tabular}


Supplementary Table 10 Univariate tests of parameter estimates of two measures of fungal growth in three fungus-growing termite species: (i) production of fungal biomass (' $\mathrm{g}$ ' after 20 days of incubation), and (ii) radial growth ( $\mathrm{cm} 2$ after 24 days of incubation) across a nutritional landscape of 36 diets varying across nine P:C ratios (between 1:9 and 9:1) and four protein and carbohydrate concentrations $(8,32,56$, and $80 \mathrm{~g} / \mathrm{L})$.

\begin{tabular}{l|lllllll}
\hline $\begin{array}{l}\text { Termite } \\
\text { species }\end{array}$ & $\begin{array}{l}\text { Depen- } \\
\text { dent } \\
\text { variable }\end{array}$ & $\begin{array}{l}\text { Degrees } \\
\text { of free- } \\
\text { dom } \\
\text { model }\end{array}$ & $\begin{array}{l}\text { Degrees } \\
\text { of free- } \\
\text { dom error }\end{array}$ & R2 & $\begin{array}{l}\text { Mean } \\
\text { square } \\
\text { model }\end{array}$ & F & P \\
\hline O. sp. & Biomass & 17 & 302 & 0.629 & 54.62 & 18.51 & $<.0001$ \\
& Area & 17 & 302 & 0.510 & 0.4149 & 30.18 & $<.0001$ \\
\hline O. cf. badius & Biomass & 17 & 302 & 0.563 & 0.4596 & 22.92 & $<.0001$ \\
& Area & 17 & 302 & 0.477 & 26.25 & 16.24 & $<.0001$ \\
\hline M. natalensis & Biomass & 17 & 302 & 0.458 & 0.3062 & 15.01 & $<.0001$ \\
& Area & 17 & 302 & 0.553 & 44.39 & 21.96 & $<.0001$ \\
& & & & & & &
\end{tabular}




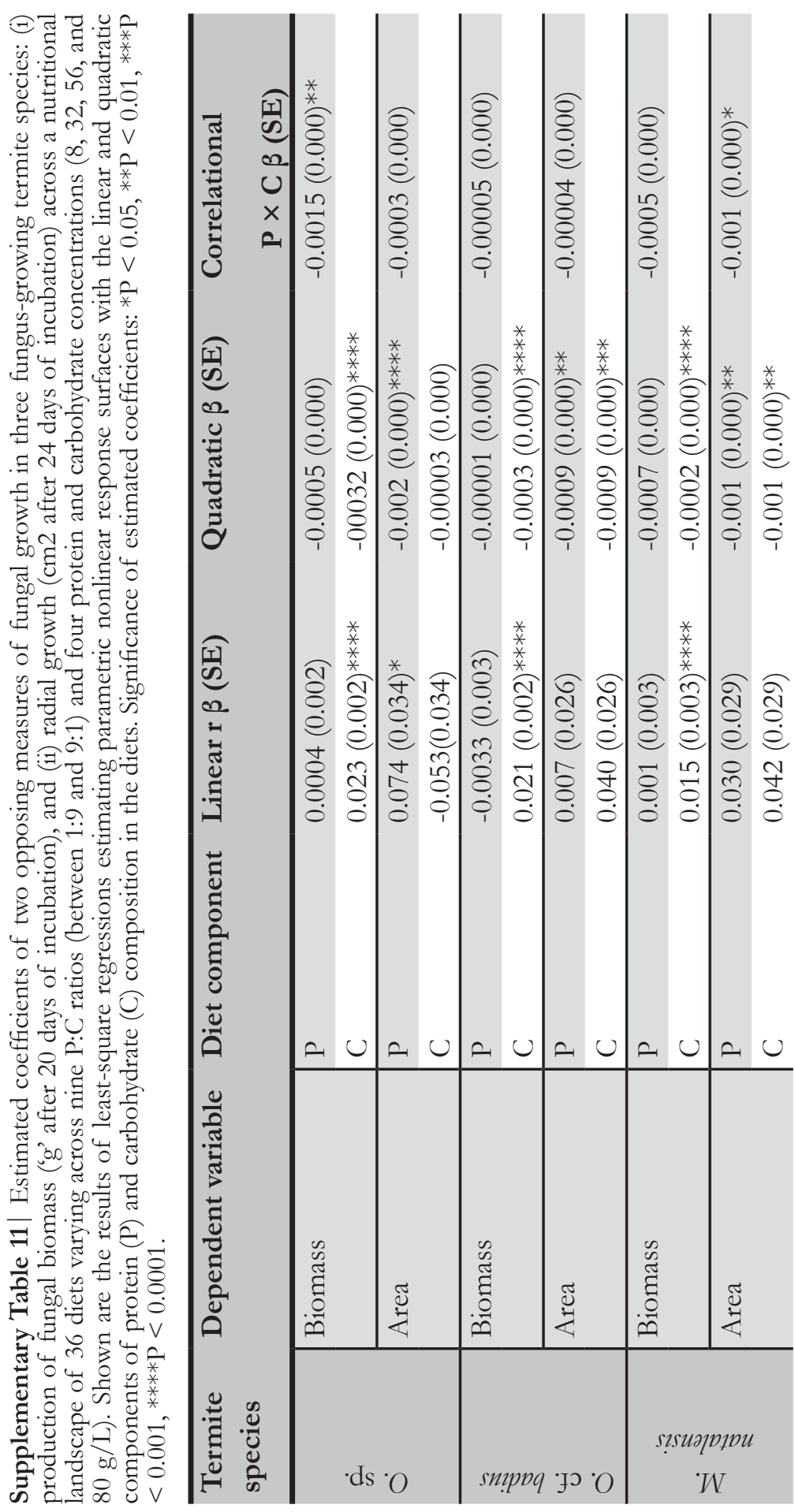


Supplementary Table 12, $13 \& 14 \mid$ can be found online at https://doi.org/10.1016/j.funeco.2018.08.009.
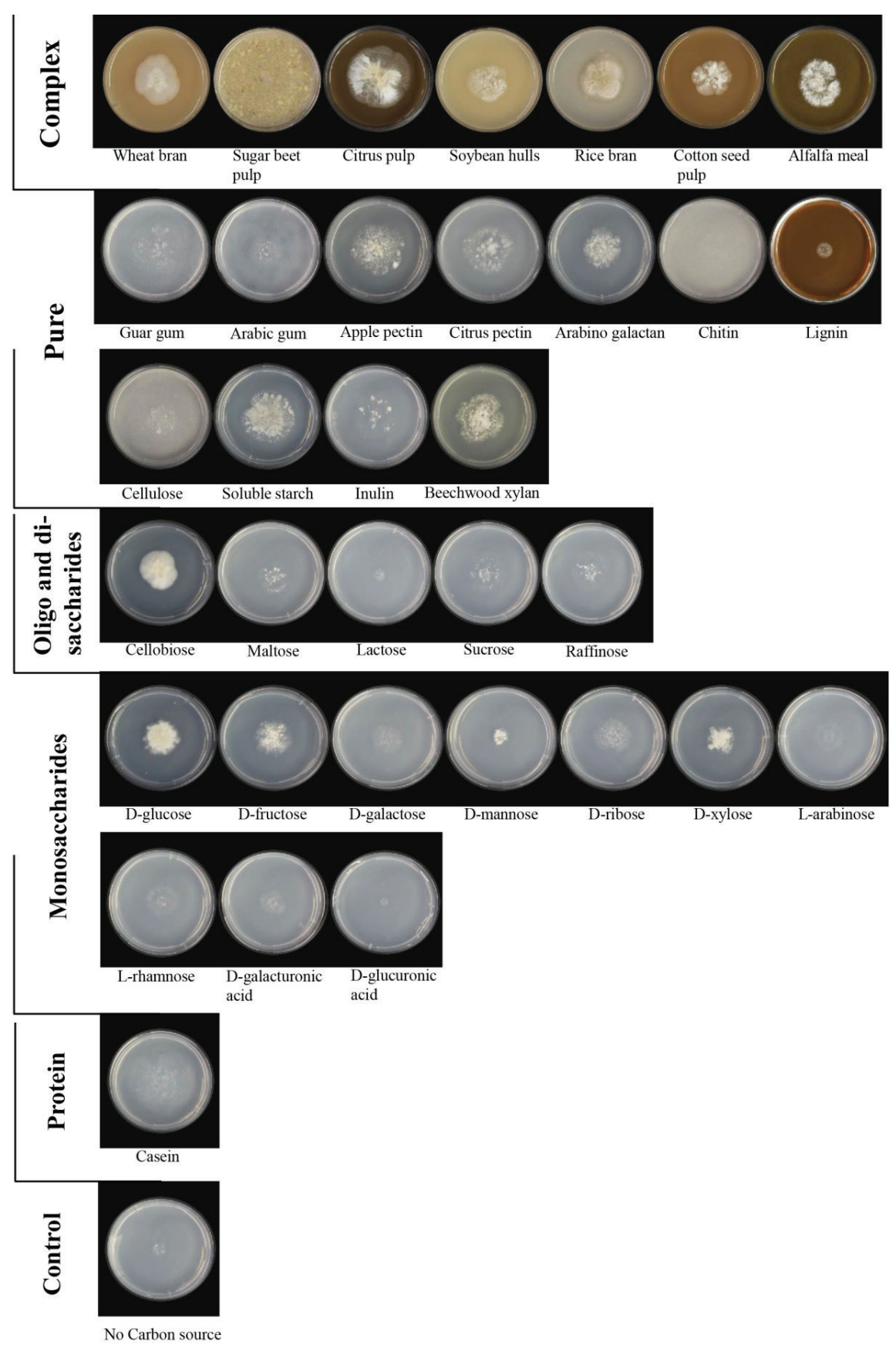

Supplementary Figure 1| Pictures of Termitomyces cultured on different carbon sources. 


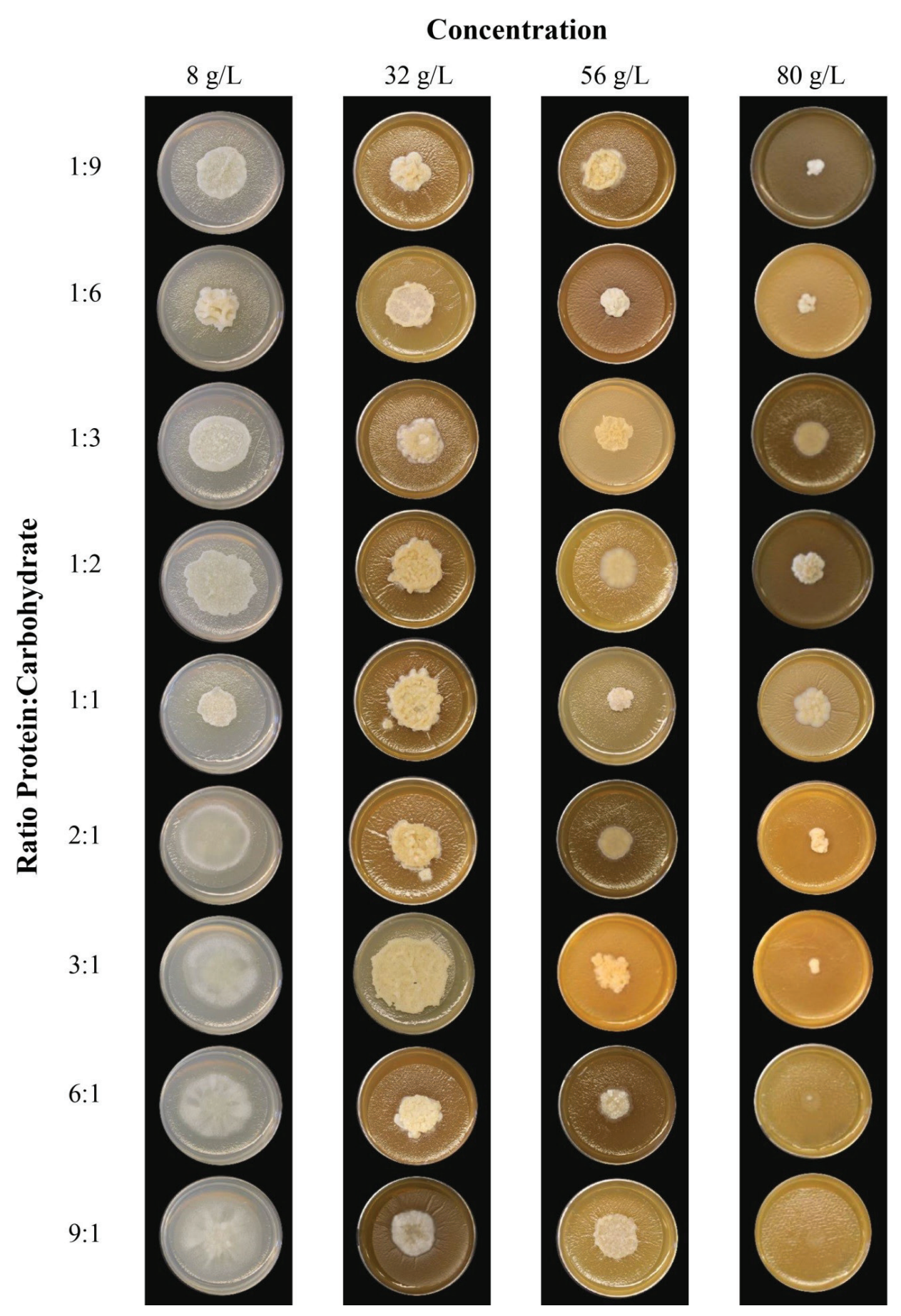

Supplementary Figure 2| Pictures of Termitomyces cultured on 36 media with different ratios and concentrations of protein and carbohydrate. 


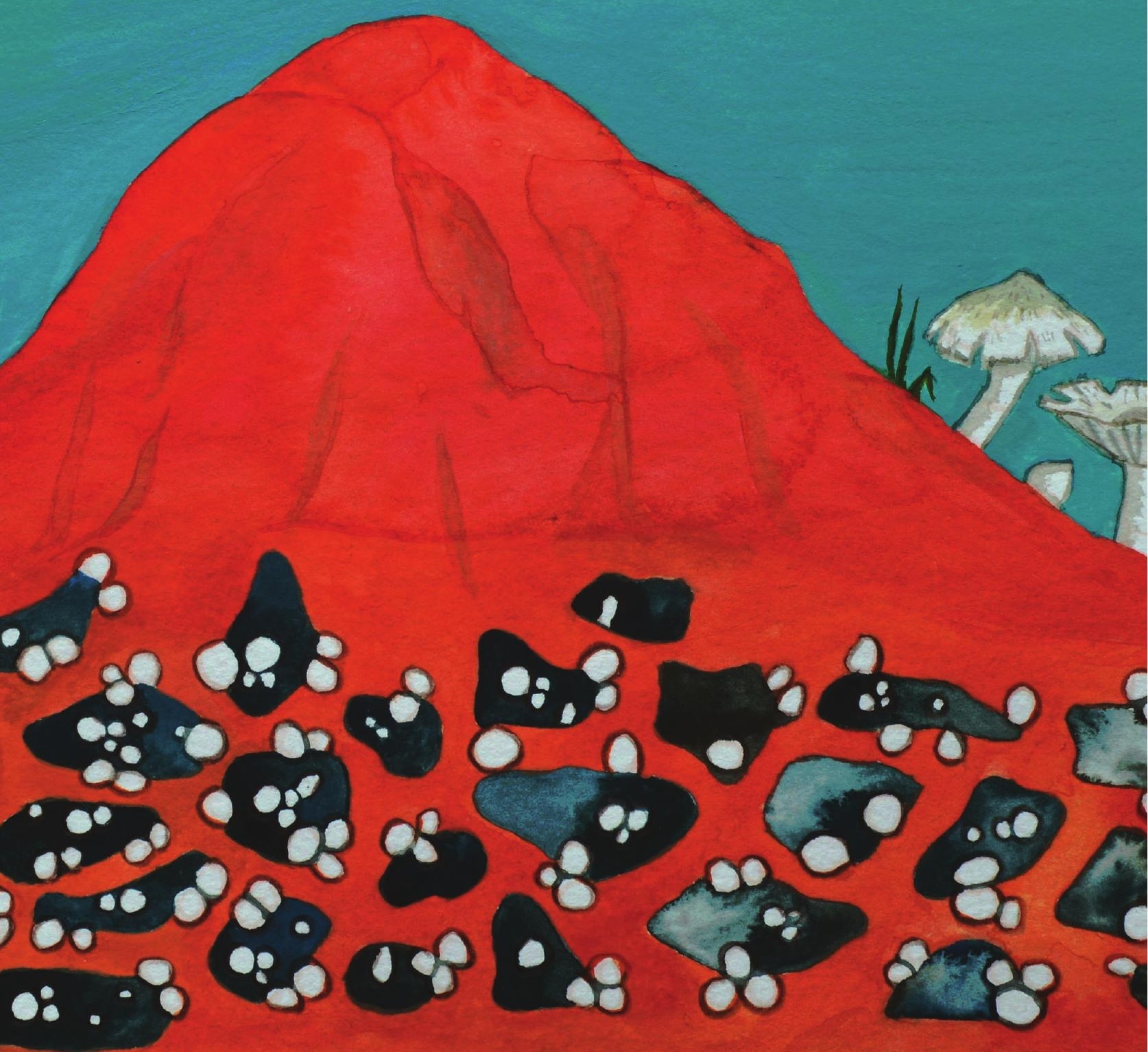



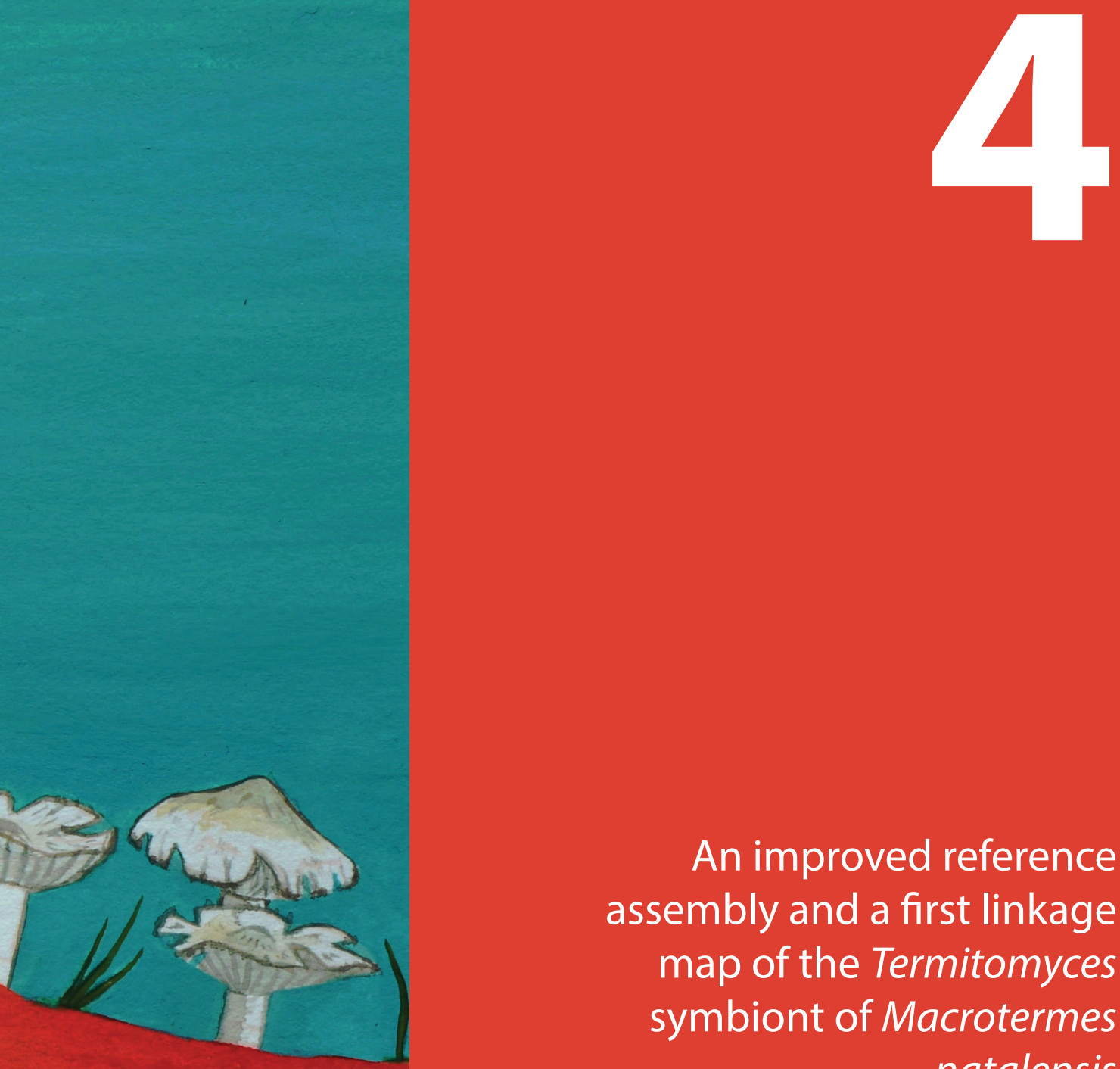

\section{An improved reference assembly and a first linkage map of the Termitomyces symbiont of Macrotermes natalensis}

Sabine M.E. Vreeburg, Bas Jacobs, Peter M. Bourke, Joost van den Heuvel, Bas J. Zwaan, Duur K. Aanen 


\section{Abstract}

The termite-fungus symbiosis is an example of an ancient stable mutualism despite the theoretically destabilising mode of horizontal symbiont transmission; each newly founded termite colony needs to re-establish the interaction with a fungal symbiont (horizontal transmission). The first assembly of the reference genome of the symbiotic partners indicated that part of the stability can be explained by genetic complementarity in the ability to break down plant substrate between the symbiotic partners. Here we present a new, more contiguous Termitomyces v2.0 reference assembly and the first linkage map of the Termitomyces symbiont of the termite Macrotermes natalensis, to aid future studies of how the mutualistic lifestyle has shaped evolution of Termitomyces genomes. In this study we focused on one characteristic that may have been affected by the mutualistic lifestyle: the recombination landscape. It has been hypothesised that inhabitant symbionts such as Termitomyces fungi that live in a stable biotic environment and have been adapted to a symbiotic lifestyle should be selected to undergo little genetic change, e.g. little recombination. Here we used the positional information of markers - created by a Genotyping-by-Sequencing approach - on the v2.0 reference assembly to compare physical to genetic distance for a first exploration of the recombination landscape. Under the assumption of randomly distributed crossovers, our mapping population showed substantial variation in recombination rate across the genome. However, to provide a conclusive answer to whether recombination rate in Termitomyces fungi is indeed lower than in free-living relatives, future research should aim to study the recombination landscape in more Termitomyces clades, as well as in their close relatives. 


\section{Introduction}

From bees and orchids, to corals and algae, to the microbes in our guts, mutualisms are ubiquitous in the world and have shaped life as we know it. One of these mutualisms that has had an important impact on Old-world tropical ecosystems is the mutualistic symbiosis between Termitomyces fungi and their termite farmers (Macrotermitinae). This obligate mutualism has allowed its partners to engineer ecosystems (Lavelle et al. 1997), to become major decomposers of the African savannahs (Wood and Sands 1978), and to incur massive agricultural losses (Rouland-Lefèvre 2011). In addition, the mushrooms that the fungus produces seasonally are considered local delicacies that contain important nutrients, including high amounts of essential amino acids (Ogundana and Fagade 1982, Botha and Eicker 1992, Kansci et al. 2003, Malek et al. 2012). In the areas where Termitomyces is found, its mushrooms are considered to be an important source of nutrition and income (Kone et al. 2011).

The termite-fungus symbiosis is an example of a symbiotic mutualism that has remained stable over 30 million years (Aanen et al. 2002, Aanen and Eggleton 2005), despite horizontal transmission of the fungal symbiont (Johnson et al. 1981, Korb and Aanen 2003, Nobre et al. 2011a). Horizontal transmission means that reproduction of the host and symbiont are not coupled, but instead each new generation needs to re-establish the interaction. The latter is an interesting feature, because theoretical studies indicate that if the reproductive interests of host and symbiont are not fully aligned, which is the case if there is horizontal transmission of the symbiont, this is a potential source of conflict (Herre et al. 1999). Mixing genetically unrelated symbionts with a single host may have direct or indirect costs due to selection for antagonistic traits that have negative side effects for the host. Therefore, the host is under selection to reduce symbiont dispersal and the associated mixing between genetically different symbionts (Frank 1996).

In addition to the conflict over symbiont mixing, an issue of horizontal symbiont transmission is that a newly founded termite colony needs to obtain the 'right' symbiont. It has been shown that there is a certain level of interaction specificity between termites and their fungi (Aanen et al. 2002, Aanen et al. 2007, De Fine Licht et al. 2007). In general termite species from one clade live together with fungal symbionts from a particular fungal clade, although within clades there are large differences in interaction specificity; some termites are only found with a single fungal species, other termites can be found with different fungal species. And from the fungal side, some fungal isolates are only cultivated by one termite species, whereas other fungal isolates can be cultivated by many termite species (Aanen et al. 2002, van de Peppel and Aanen 2020). The factors underlying the observed interaction specificities are currently unknown, although 
it has been hypothesised that different complementarities in substrate breakdown and differences in the degree of complementarity may play a role (Rouland-Lefèvre 2000, De Fine Licht et al. 2007, Nobre and Aanen 2012, da Costa et al. 2019). In 2014 the genomes of a Termitomyces fungus, its termite farmer and the termite's gut microbiota were published (Poulsen et al. 2014). These reference sequences allowed Poulsen et al. to show that the symbiotic partners have indeed developed some complementarity to break down the complex substrate that they feed on, thereby ensuring faithfulness and enhancing the stability of the symbiosis. Whether there are genetic differences in the extent of complementarity between termite-fungus combinations remains to be systematically tested.

In the case of fungus-growing termites horizontal symbiont acquisition also involves sexual reproduction of the fungal symbiont through the formation of mushrooms. Within a termite colony a single fungal heterokaryon is propagated vegetatively. After a termite colony produces dispersing reproductives, its fungus produces mushrooms that spread sexual spores in the environment that can inoculate newly founded colonies (Sands 1960, Johnson et al. 1981, Sieber 1983, Korb and Aanen 2003, De Fine Licht et al. 2006, Kone et al. 2011). Law and Lewis (1983) hypothesised that sex, which they define as any process involved in reshuffling chromosomal genomes, should be selected against in inhabitant mutualistic symbionts because they live in a sheltered biotic environment to which they have co-evolved. Also, Bergstrom and Lachmann (2003) showed that mutualistic interactions often favour slow rates of evolution, in contrast to the rapid evolution that is found in antagonistic relationships. Following from the work of Law and Lewis and Bergstrom and Lachmann, we hypothesise that the sexual recombination rates of the inhabitant Termitomyces fungi should be lower than that of their free-living relatives.

To be able to study recombination and differences in complementary, high quality, contiguous reference assemblies are needed. The emergence and further development of next-generation sequencing technologies have revolutionised genetic research. Currently, genetic sequences are publicly available even for non-model species and many more will be added over the coming years. However, many of the reference genomes are based on cheaper, short-read technologies, such as the Termitomyces v1.0 reference assembly (Poulsen et al. 2014), while more repetitive regions of the genome are almost impossible to assemble with short reads. As evidence is accumulating that especially these repetitive regions are more subject to genomic changes in eukaryotic genomes and thus of high interest when studying the evolution of related species, it would be highly beneficial to have more complete reference assemblies of the species we are studying (Thomma et al. 2016). Also, more contiguous reference assemblies would allow for a 
plethora of studies including QTL analyses, analysis of gene clusters rather than single genes, or synteny analysis (Lewin et al. 2009, Thomma et al. 2016).

In this study we focused on the study of recombination in the Termitomyces symbiont of Macrotermes natalensis. We first made a new version of the Termitomyces reference assembly using long-read sequencing technology. To allow for comparison of genetic studies on the v1.0 assembly to the new assembly we transferred the v1.0 annotation to the v2.0 reference assembly. Subsequently, we constructed the first genetic linkage map of a Termitomyces species using SNP markers generated by Genotyping by Sequencing (GBS) on single-spore isolate cultures from the mushroom of a single Macrotermes natalensis colony (Elshire et al. 2011). Finally, to analyse the recombination landscape of the Termitomyces symbiont of $M$. natalensis, we used the positional information of the SNP markers on the v2.0 assembly to compare the physical to the genetic distance.

\section{Material and Methods}

\section{Genome assembly:}

\section{Culture and DNA isolation}

A homokaryotic strain of the Termitomyces symbiont of $M$. natalensis was previously obtained through protoplasting by Poulsen et al. (2014). The fungus was grown in liquid culture in Malt Yeast (MY) medium (20g malt, $2 \mathrm{~g}$ yeast, $1 \mathrm{~L}$ demineralised water) and freeze dried. DNA was extracted using a CTAB protocol. Removal of RNA contamination, library preparation and sequencing were performed by BGI. Sequencing was done on the Pacbio Sequel System.

\section{Assembly of genome v2.0}

Pacbio reads were assembled using the CANU assembler v1.7 (Koren et al. 2017) using the estimated genome size of Poulsen et al. $(83.7 \mathrm{Mb})$ for the parameter genomeSize (Poulsen et al. 2014). Previously published Illumina data (Poulsen et al. 2014) was trimmed for quality in CLC Genomics Workbench v9 using default settings. The trimmed reads were used to polish the CANU assembly with Pilon v1.22 (Walker et al. 2014) using default settings. Genome statistics were obtained by QUAST v4.6.3. (-m 0 to be able to compare to v1.0 genome statistics) (Gurevich et al. 2013) and assembly completeness was assessed using BUSCO v3, gene set Basidiomycota odb9) (Simao et al. 2015). Pacbio reads were aligned back to the genome using minimap2 v2.17-r954dirty (Li 2018) and average alignment depth was calculated using samtools v1.7 (Li et al. 2009). The assembly was aligned to the previous version (v1.0) of the reference genome of Termitomyces (Poulsen et al. 2014) using minimap2 v2.17 (Li 2018). The alignment was visualised using dotPlotly (-q 1000, -m 1000) (https://github.com/tpoorten/dotPlotly). The mitochondrion contig was identified using BLAST (online) (Nieuwenhuis 
et al. 2019). Annotation of TIG004 in our assembly was lifted over from the annotation of assembly v1.0 using the "Live Annotate \& Predict" function in Geneious v10.0.9 with a similarity of $99 \%$ (Kearse et al. 2012).

\section{Linkage mapping: Mapping population}

A population of homokaryotic siblings was obtained from mushrooms of a single Macrotermes natalensis colony in Modimolle, South Africa (GPS: S24 40.484 E28 48.271). Mushrooms were obtained by incubation of fungal combs (Vreeburg et al. 2020). Stems of the mushrooms were cut away close to the gills and the caps were attached to the lids of Petri dishes using dots of Vaseline. Spores were deposited on Petri dishes containing Malt Yeast Agar (MYA: 20g malt, $2 \mathrm{~g}$ yeast, 15g Agar, 1L demineralised water) for a range of time periods varying from a few seconds, to 24 hours. Petri dishes with spore prints were incubated at $25^{\circ} \mathrm{C}$ and monitored daily for hyphal growth under a binocular. To isolate homokaryons, small patches of hyphal growth were isolated using a needle and placed on a fresh plate of MYA.

\section{DNA isolation}

All isolates were grown in liquid culture in Malt Yeast medium. DNA was isolated from the putative single spore cultures and the parental heterokaryon using a CTAB extraction. Any RNA present in the samples after extraction was degraded by $3 \mu \mathrm{l}$ RNase I (Thermo Scientific) incubated for two hours at $37^{\circ} \mathrm{C}$.

As the colonies were isolated as soon as hyphal growth was observed, we expected most of the mapping isolates to be homokaryotic, but some may have formed heterokaryotic mycelium by fusion of the hyphae of two adjacent compatible germinating spores. As Termitomyces species do not form clamp connections there is no clear distinction between homokaryons and heterokaryons (De Fine Licht et al. 2005). However, Termitomyces heterokaryons have been shown to have higher growth rates than homokaryons, enabling a rough selection of putative homokaryons based on growth compared to the heterokaryon (Nobre et al. 2014). To test whether our selection of putative homokaryons vs. heterokaryons was successful, we developed a Cleaved Amplified Polymorphic Marker (CAPS) marker on a highly variable part of the nuclear Elongation Factor 1 alpha gene for the parent heterokaryon. We amplified this region using primers EF595F and EF1160R (De Fine Licht et al. 2006) and Sanger-sequenced the product. A double peak in the chromatogram showed a disrupted NdeI restriction site for one of the two alleles. Using this marker 37 isolates were analysed: twelve suspected heterokaryons and 25 putative homokaryons (Supplementary Figure 1). Since none of the putative homokaryons and roughly half of the suspect heterokaryons showed heterozygosity (which would be expected if all of them were heterokaryons resulting from a mating of sibling homokaryons), these 12 along with all other suspected heterokaryons in the 
mapping population were excluded from the further analyses. From the remaining presumed homokaryons in the mapping population, 92 were chosen at random for GBS analysis. In addition, the parent heterokaryon was included in three replicates as a control.

\section{Genotyping-by-sequencing (GBS)}

GBS was performed at the Genomic Diversity Facility of Cornell University according to the protocol described by Elshire et al. (2011). The enzyme used for the restriction step was EcoT22I (a six-base cutter) rather than ApeKI (a five-base cutter with one wobble base), because the less frequent cutting results in fewer different sequenced fragments and therefore higher coverage per sequenced fragment. Although this does decrease the number of SNPs that will be found, it increases the chance that most individuals can be scored for a certain marker, which is favourable for linkage analysis.

\section{The parent heterokaryon: protoplasting, sequencing, and assembly}

To reconstruct the parental genotype to identify marker phasing and to compare the genome of the parent to our reference assembly we wanted to obtain the constituent nuclei of the parent heterokaryon. One gram of mycelium (wet weight) from a liquid culture of the parent heterokaryon was crushed in $1 \mathrm{~mL}$ saline solution $(8 \mathrm{~g} / \mathrm{L} \mathrm{NaCl})$ to obtain a homogeneous suspension. $1 \mathrm{~mL}$ of this suspension was added to $100 \mathrm{ml}$ MY medium in a sterile $500 \mathrm{ml}$ Erlenmeyer flask and incubated for one week at $25^{\circ} \mathrm{C}$, shaking at $100 \mathrm{rpm}$. Mycelium was harvested using a sterile Büchner funnel with a sterile nylon filter, washed with $0.6 \mathrm{M}$ sucrose. $1.2 \mathrm{~g}$ of mycelium (wet weight) was added to $10 \mathrm{~mL}$ of protoplasting solution $(0.6 \mathrm{M}$ sucrose and $20 \mathrm{~g} / \mathrm{L}$ Novozym 234 (Novo Nordisk), filter sterilised) and incubated for $2-3$ hours at $30{ }^{\circ} \mathrm{C}$, shaking horizontally at $80 \mathrm{rpm}$.

The resulting mixture with protoplasts was filtered over a glass wool plug in a funnel pre-rinsed with $0.6 \mathrm{M}$ sucrose into a fresh tube, after which the filter was rinsed again with $0.6 \mathrm{M}$ sucrose. The protoplasts in the filtrate were collected by centrifugation (10 $\mathrm{min}, 2000 \times \mathrm{g}, 10^{\circ} \mathrm{C}$ ). Protoplasts were resuspended in $5 \mathrm{~mL} 0.6 \mathrm{M}$ sucrose and collected again by centrifugation $(5 \mathrm{~min}, 3000 \times \mathrm{g}$, room temperature). Protoplasts were resuspended in $5 \mathrm{~mL} 0.6 \mathrm{M}$ sucrose, after which their concentration was determined using a Neubauer haemocytometer (Brand GmbH + Co KG). The mixture was then again centrifuged ( $5 \mathrm{~min}, 3000 \times \mathrm{g}$, room temperature), the supernatant was discarded, and the protoplasts were resuspended in $0.6 \mathrm{M}$ sucrose to a concentration of approximately $5 \times 106$ protoplasts $/ \mathrm{ml} .1 / 10$ and $1 / 100$ times dilutions were made of which 100 $\mu \mathrm{l}$ was plated on MYA with 0.6 M sucrose. Regeneration plates were incubated for six days at $25^{\circ} \mathrm{C}$. From the plates where regeneration had been successful after the incubation period, individual colonies developing from regenerated protoplasts were transferred to fresh MYA plates, which were incubated at $25^{\circ} \mathrm{C}$. The resulting cultures were 
analysed with the CAPS marker described above. Most recovered protoplasts were heterokaryotic, and all recovered homokaryotic cultures possessed one of the two nuclei, with the allele without the NdeI restrictions site. Whole-genome Illumina sequencing was performed of this homokaryotic culture, $\mathrm{mt} 50 \mathrm{a}$ (one of the parent homokaryons). Sequences were quality filtered using Trim Galore! (Galaxy version 0.6.3) and assembled using SPAdes with default parameter settings v3.12.0 (Bankevich et al. 2012). The $\mathrm{mt50a}$ assembly was aligned to the our reference assembly v2.0 using minimap2 2.17 (Li 2018). The alignment was visualised using dotPlotly (-q 500, -m 500) (https://github. com/tpoorten/dotPlotly).

\section{SNP discovery}

The GBS reads were demultiplexed using sabre version 1.000 and, together with the raw Illumina reads of $\mathrm{mt50a}$, aligned to the $\mathrm{v} 2.0$ genome using the BWA-MEM algorithm v0.7.17 (Li 2013). SNPs were called using Freebayes v1.3.1 (Garrison and Marth 2012) with a minimum read depth $(-C)$ of 5 .

\section{Strict SNP filtering}

For filtering of the VCF file the TASSEL GUI v 5.2 .58 was used. We initially filtered by position: min individuals called $=40$, min allele frequency $(\mathrm{AF})=0.2, \max \mathrm{AF}=0.8$, max heterozygous calls $=0.1$. Then, to filter out heterokaryons, we filtered out all samples with more than $10 \%$ heterozygous SNPs. Subsequently, we repeated the first filter by position, set all heterozygous calls to unknown and removed the blank control. Next, we removed all positions with three alleles, all positions that were missing in the mt50a parent, all positions with more than $10 \%$ missing and the positions with a cumulative $\mathrm{p}$-value of the observed minor allele frequency $<0.01$ (under a binomial distribution). Then we removed those positions that had SNP calls that were not supported by adjacent markers, i.e. SNP calls that would require a double crossover. Finally, we used the R package polymapR v.1.0.20 (Bourke et al. 2018) to filter out positions with the function checkF1 (qall_weights $<0.75$ ), individuals with more than $10 \%$ missing positions and positions that had less than 5 unique markers in a linkage group at LOD $>5.5$.

\section{Linkage mapping}

To make a linkage map we used the $\mathrm{R}$ package polymapR in Rstudio v3.6.1 (R Core Team 2019), with a dummy "nulliplex" parent added, to make it suitable for our mono-parental mapping population (analogous to a back-cross population with a completely homozygous recurrent parent). Linkage groups were assigned with a LOD score $>5.5$. The linkage map was visualised using the $\mathrm{R}$ package LinkageMapView (Ouellette et al. 2018). 


\section{Mapping the mating type}

Crosses were set up between 29 of the individuals of our mapping population on Petri dishes with MYA. Six individuals were crossed against all 29 individuals (Supplementary Table 1). In the absence of clamp connections, we determined mating success by assessing changes in the interaction zone as described by Nobre et al. (2014) after four weeks of growth. To further clarify growth characteristics, a piece of mycelium was transferred from both homokaryons and the interaction zone of each cross (Supplementary Figure 2). The genotypic data were added as a binary trait $(0 / 1)$ to the strictly filtered dataset and linkage mapping was performed as described above to map the mating type locus. The mating type locus was added to the map after making the core map with strictly filtered data, because it was only scored for 29 individuals.

To confirm the resulting map position of the mating-type locus, the predicted HD1 and HD2 proteins from Schirophyllum commune H4-8 (XP_003038830.1 and XP_003037496.1 respectively) were blasted against the genome using Protein Query-Translated Subject BLAST 2.9.0+ (Altschul et al. 1990). To find the putative location of the pheromone/ pheromone-receptor, the $S$. cerevisiae pheromone receptor Ste3 (sp | P06783) was blasted against the v2.0 assembly.

\section{Recombination Frequency}

\section{Less strict SNP filtering}

We initially removed all individuals that the previous filter indicated as heterokaryons, and then filtered by position (minimum individuals called $=10$, minor allele frequency $(A F)=0.05)$. Then we removed all positions that had a heterozygous call for the $\mathrm{mt50a}$ parent and all positions that had more than one heterozygous call over all samples. We changed all remaining heterozygous calls to missing. We removed all positions with one or three alleles and removed positions with more than 30 missing calls. We manually curated the marker set by looking at the alignments of suspect markers in IGV (Robinson et al. 2011). In this dataset 180 extra markers were included, that had a minor allele frequency with an expected cumulative $\mathrm{p}$-value $<0.01$ under a binominal distribution that had been filtered out in the strictly filtered dataset.

\section{Forced order linkage map}

We used the linkage function of polymapR to calculate the extent of linkage between the first two and last two markers of each scaffold in the less strictly filtered genotyping data. We connected all contigs of which the most distal markers were in LD with a LOD score above 5.5. We imputed missing values by replacing any missing data point with the previous allele, unless it was at the start of a contig, in which case we used the next data point. We calculated recombination frequency to obtain a forced order linkage 
map and compared the linkage groups to the linkage groups of the linkage map that was constructed with the strictly filtered dataset using the function compare_maps of polymapR.

\section{Genome coverage estimation and marker distribution}

Total coverage of the genome by markers on the linkage map was estimated in two different ways. Firstly, the combined length of all scaffolds represented on the linkage map was calculated for both the strict linkage map and the forced order linkage map. Secondly, the physical map length represented by the linkage map was estimated by determining the distances between the first and last marker on each contig. For all values an average genetic to physical distance ratio was calculated $(\mathrm{cM} / \mathrm{Mb})$.

\section{Comparison physical and genetic distance in the forced order map}

To examine the relation between physical and genetic distance, genetic positions were plotted against physical positions for all linkage groups in the forced-order linkage map. To assess whether there were intervals with more or less recombination compared to the average number of crossovers, we first examined whether there was a correlation between the number of crossovers in an interval between markers and the number of recombination events between those markers (Figure 1A). Spearman's correlation between physical distance and the number of recombination events was 0.46 ( $\mathrm{p}$-value $<2.2 \mathrm{e}-16$ ). We constructed a probability distribution, given the marker distance, i.e. the probability of finding a recombination event in the mapping population between two markers is equal to the marker distances between these two markers divided by the sum of all marker distances in our map. Then we drew 10,000 multinominal random samples, with probability as explained above. For each interval between two markers we compared the number of crossovers in our population to all random samples and calculated the probability of finding more or fewer crossovers than the observed number of crossovers. We corrected the obtained p-values for multiple testing using a Benjamini-Hochberg procedure (Benjamini and Hochberg 1995).

As marker distribution is not evenly spread across our assembly (Figure 1A) we repeated the above described test with our markers grouped in distances of approximately $500,000 \mathrm{bp}$ (Figure 1B). We chose this value because only $2.8 \%$ of the marker distances are larger than $500,000 \mathrm{bp}$. Also, looking at larger regions of the genome is more reliable for finding regions with low recombination frequencies.

\section{Results}

\section{V2.0 Genome assembly}

Pacbio data consisted of 666,235 reads, with a total length of 4,711,826,269bp. The largest read was 76,016bp and the average read length was 7,072bp. Based on the ge- 
A

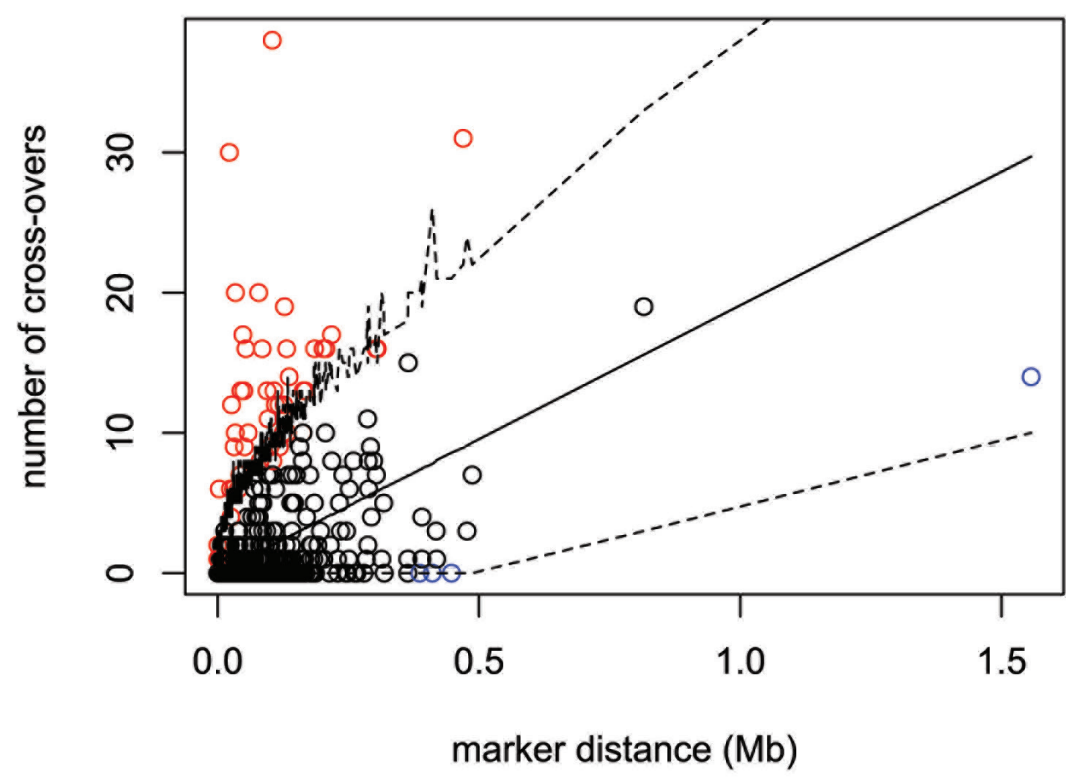

B

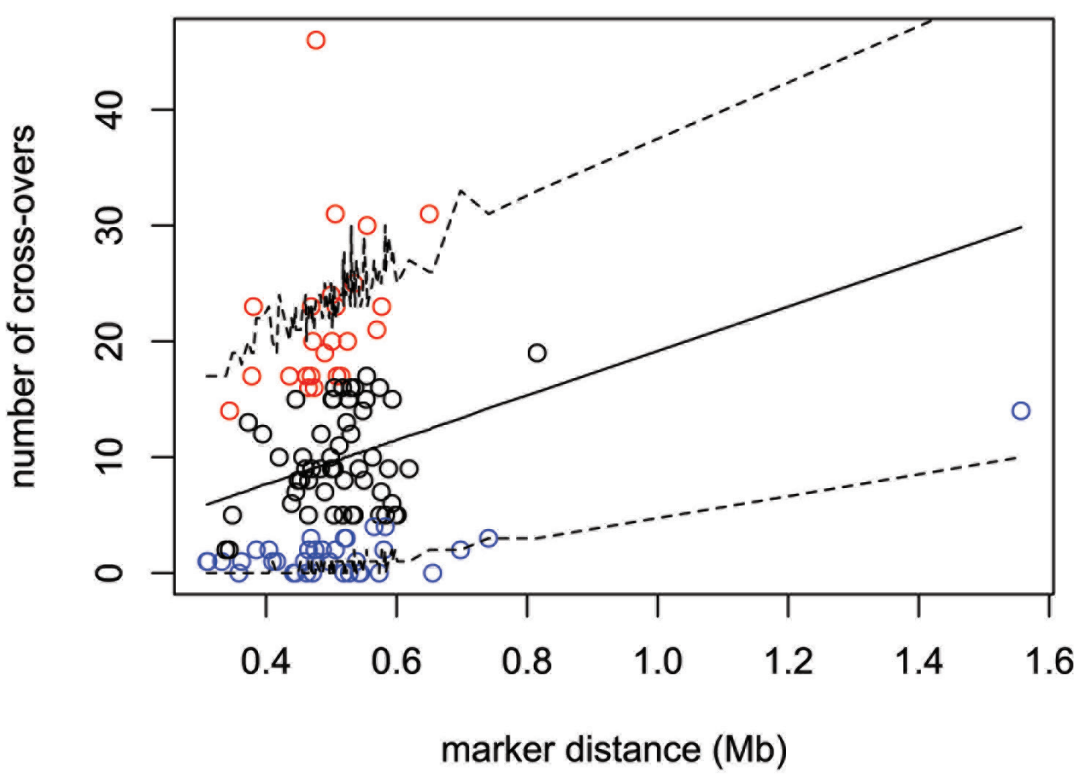

Figure 1| Number of crossovers (y-axis) plotted against marker distance between two adjacent markers. On the left, A, non-grouped marker distances, i.e. markers spread unevenly over the genome. On right, B, the intervals of grouped markers in regions of approximately 500000bp. Red dots are significantly more recombination then expected compared to the simulated datasets, blue spots significantly less recombination ( $<\mathrm{p}$-value 0.05$)$. Lines give maximum, average, minimum recombination in simulated datasets. On the left non-grouped marker distances, i.e. markers spread unevenly over the genome. On the right markers grouped in regions of approximately 500000bp. 
nome size estimate of Poulsen et al. (2014), this indicated a coverage of 56x, which is in agreement with the average alignment depth of Pacbio reads which was 56.8. Our v2.0 assembly of the Termitomyces genome consisted of 64 contigs and totalled a little over 70 $\mathrm{Mb}$ (Table 1). Compared to the v1.0 reference assembly the N50 increased over 10-fold. However, our v2.0 assembly only contains contigs; CANU only produces contigs, not scaffolds. When we compare the N50 of the v2.0 assembly to the N50 of the contigs of the v1.0 assembly, the N50 increased 100-fold. By blasting the mitochondrion sequence (Nieuwenhuis et al. 2019) against our v2.0 assembly, one of the contigs (TIG068) was identified to represent the mitochondrion. BUSCO analyses revealed that $96.6 \%$ of the BUSCO gene set of Basidiomycota was present in the assembly (Table 1). After the first polish with Pilon we did a second iteration of Pilon polishing on this version. As the BUSCO score did not change with the second polish, we decided to use the version that was polished once with the Illumina reads from Poulsen et al. (2014) for further analysis.

Table 1| Summary statistics for the v1.0 Termitomyces reference genome, the assembly with only raw Pacbio reads (assembled by CANU), the v2.0 CANU assembly polished by Pilon with Illumina reads, and the SPAdes assembly of the $\mathrm{mt} 5 \mathrm{O}$ a used as parent for mapping population.

\begin{tabular}{lllll}
\hline & $\begin{array}{l}\text { v1.0 assem- } \\
\text { bly (Poulsen } \\
\text { et al. 2014) }\end{array}$ & $\begin{array}{l}\text { CANU } \\
\text { assembly }\end{array}$ & $\begin{array}{l}\text { v2.0 assembly: } \\
\text { CANU assembly, } \\
\text { polished with Pilon }\end{array}$ & $\begin{array}{l}\text { Mt50a } \\
\text { SPAdes } \\
\text { assembly }\end{array}$ \\
\hline $\begin{array}{l}\text { QUAST } \\
\text { analysis: }\end{array}$ & & & & \\
\hline scaffolds & 11244 & 64 (contigs) & 64 (contigs) & 50520 \\
\hline $\begin{array}{l}\text { Total length } \\
\text { (bp) }\end{array}$ & $68,490,755$ & $70,033,428$ & $70,034,757$ & $70,243,221$ \\
\hline N50 (bp) & 262,000 & $2,721,236$ & $2,721,287$ & 43,018 \\
\hline N75 (bp) & 135,874 & $1,707,221$ & $1,707,259$ & 13,025 \\
\hline
\end{tabular}

\begin{tabular}{|lllll}
\hline $\begin{array}{l}\text { BuSCO } \\
\text { analysis: }\end{array}$ & & & & \\
\hline Complete & $94.2 \%$ & $96.3 \%$ & $96.6 \%$ & $96.3 \%$ \\
\hline Single & $92.9 \%$ & $94.7 \%$ & $95 \%$ & $95.1 \%$ \\
\hline Duplicated & $1.3 \%$ & $1.6 \%$ & $1.6 \%$ & $1.2 \%$ \\
\hline Fragmented & $2.3 \%$ & $1.7 \%$ & $1.6 \%$ & $1.6 \%$ \\
\hline Missing & $3.5 \%$ & $2 \%$ & $1.8 \%$ & $2.1 \%$ \\
\hline
\end{tabular}


Alignment of the v1.0 assembly to our assembly showed that every scaffold ( $>200 \mathrm{bp}$ ) in the v1.0 assembly was present in our v2.0 assembly. Reversely, only the two smallest contigs from our v2.0 assembly were not present in the v1.0 assembly (Supplementary Figure 3).

\section{Annotation liftover}

After liftover of the annotation from the v1.0 assembly to the v2.0 assembly, $91.1 \%$ of the 11556 originally predicted mRNA geneIDs were lifted over, of which $98.0 \%$ was found in a single location. Of the IDs that were found in multiple locations, most $(61.5 \%)$ were found in two locations. Fungus_00540 was found the most, on 21 locations.

\section{Putative telomeres and ribosomal DNA}

A manual search for putative telomeres in assembly v 2.0 revealed a reoccurring 6bp repeat, CACTAA, at the start of a contig or its reverse complement at the end. We found this repeat on the ends of 15 of the contigs in our assembly. The sequence was repeated 13.4 times on average, the minimum repeat number was four and the maximum 21.

Blasting ribosomal subunits $5 \mathrm{~S}, 5.8 \mathrm{~S}, 28 \mathrm{~S}$ and $18 \mathrm{~S}$ revealed that the ribosomal subunits of this Termitomyces species are clustered together with the $5 \mathrm{~S}$ subunit between the $18 \mathrm{~S}$ and 28 S subunits. The two smallest contigs (TIG00019587 and TIG00079588) only contained ribosomal repeats (two times completely and four times incompletely, respectively), and contig TIG015 contained each ribosomal subunit one time at its start.

\section{Linkage mapping}

GBS yielded on average 2.9 million reads per sample, with a standard deviation of 1.1 million, a minimum of 0.8 million, and a maximum of 5.9 million reads. The blank control sample yielded 6011 reads. Freebayes SNP calling on the GBS output and the Illumina sequencing of mt50a yielded 1,019,581 markers. After filtering, a total of 1,417 high-quality markers and 87 individuals from the mapping population were used for linkage mapping. The removal of the parent heterokaryon control samples by our filtering indicates that we successfully filtered out heterokaryotic samples. It is, therefore, likely that four other samples that were removed by filtering samples with more than $10 \%$ heterozygous SNPs, were missed by the initial phenotypic screening of the mapping population. Finally, we removed one other sample because it had too many missing calls.

Linkage mapping resulted in 13 linkage groups (LGs) with a total map length of 1,101.3 cM. In total 27 contigs from our assembly were represented in the linkage map (Figure 2, Table 2 and Supplementary Tables 2 and 3). The total length of the represented contigs was $61.4 \mathrm{Mb}$, which is $87.7 \%$ of the assembly. The sum of physical lengths between 


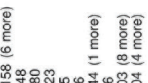

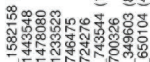

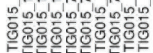

ఏิ

$\prod_{\substack{1 \\ \text { ond }}}$
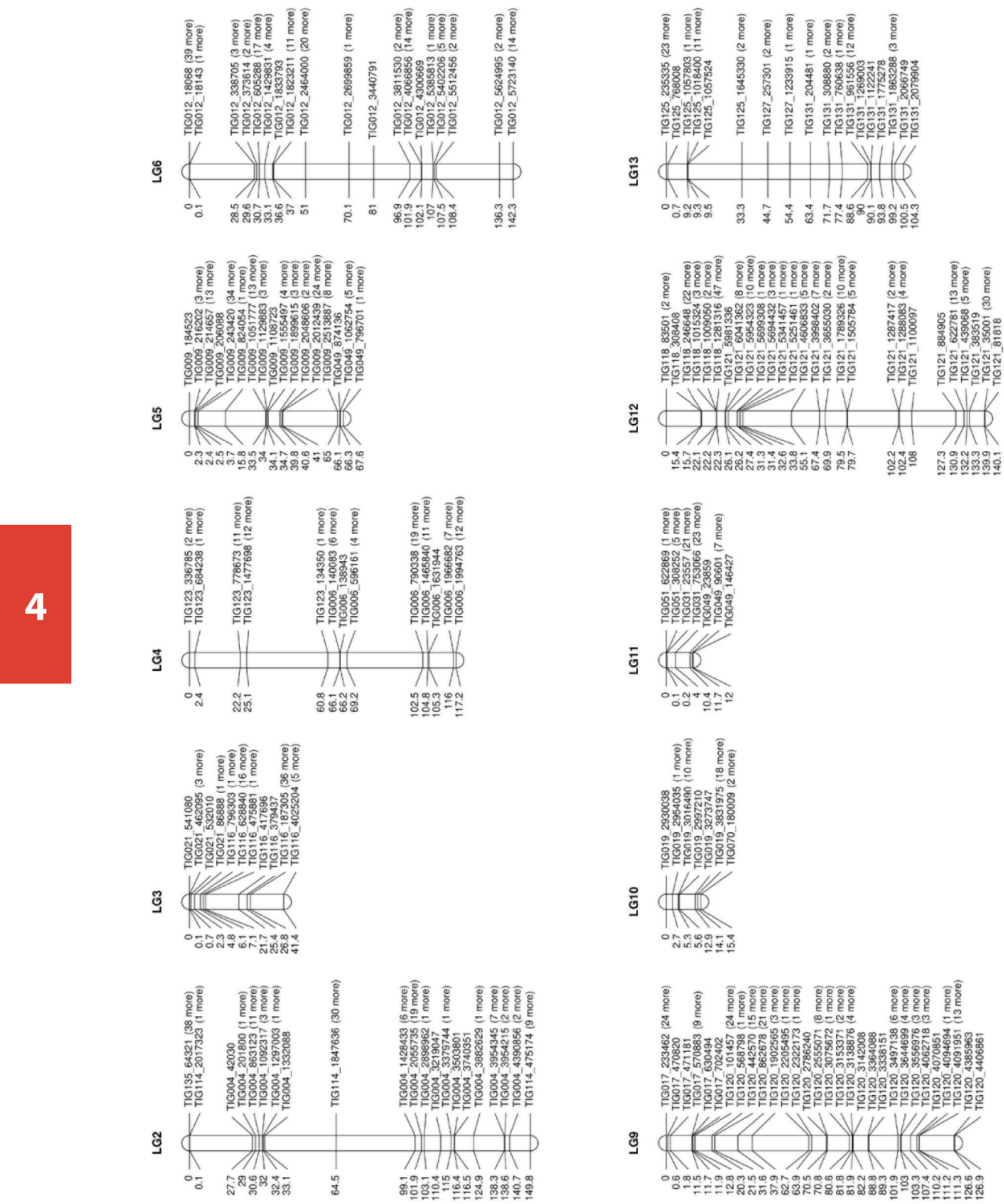

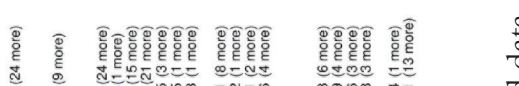

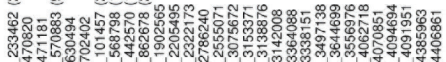

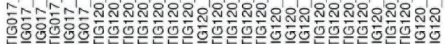

g

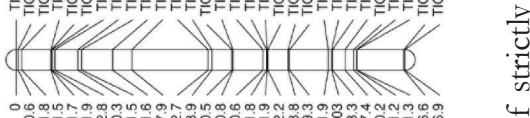
는

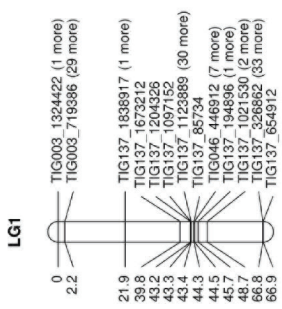

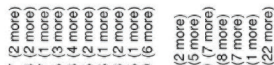

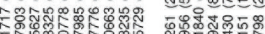

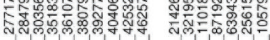

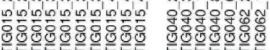

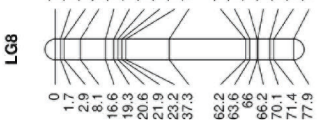


markers is $50.0 \mathrm{Mb}$, which is $71.4 \%$ of the total assembly. All markers from the same contig mapped to identical LGs except for two of the contigs from our assembly that were assigned to two different linkage groups (TIG015 and TIG049) (Supplementary Table 2). Both contigs have a large region without markers, i.e. $470 \mathrm{~Kb}$ (TIG049) and $897 \mathrm{~Kb}$ (TIG015) relative to the average marker distance (37Kb, sd 123Kb) that separates the two parts on each linkage group.

\section{Mapping the mating type}

Crosses between 30 of the single spore isolates, of which 29 are in our mapping population, indicate that the Termitomyces symbiont of M. natalensis has a bipolar mating system, as the studied homokaryons are either compatible or incompatible (Supplementary Table 1). Fourteen of the homokaryons have the one mating type allele and sixteen the other. The mating type mapped to LG2 (with a LOD score of 7.5 and 8.7 with its neighbouring markers) and fit into the pre-existing map without significantly distorting or lengthening the map (Figure 3).

\section{LG2}

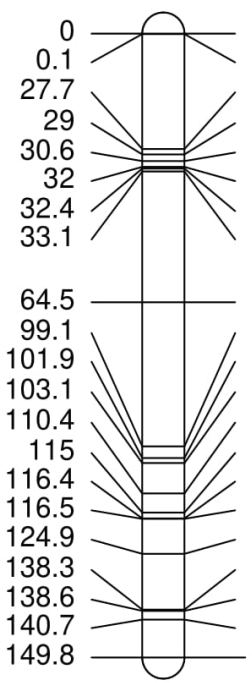

\section{LG2 with Mating Type}

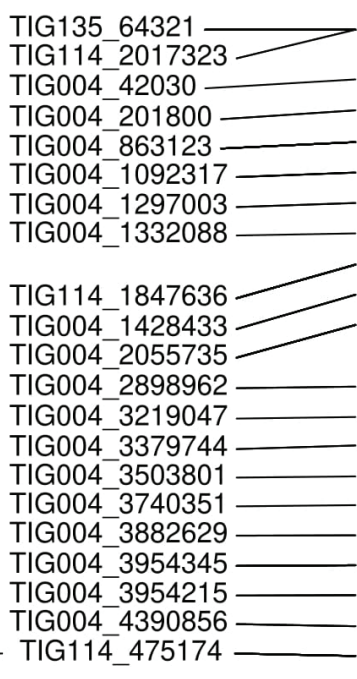

TIG135_64321 TIG114_2017323

TIG004 42030

TIG004_201800

TIG004_863123

TIG004 1092317

TIG004_1297003

TIG004_1332088

TIG114_1847636

TIG004_1428433

TIG004_2055735

Mating Type

TIG004_2898962

TIG004_3219047

TIG004-3379744

TIG004_3503801

TIG004-3740351

TIG004 3882629

TIG004_3954345

TIG004 3954215

TIG004_4390856

TIG114_475174

Figure 3| LG2 without (left) and with Mating type with lines between identical markers.

Protein to nucleotide blast of the HD1 and HD2 genes from Schizophyllum commune to the v2.0 assembly confirmed that the HD locus of the mating type is found on LG2, TIG004 in the region that was predicted by the linkage map. However, the blast results indicate that there may be two HD loci in this species. The HD1 protein was found on TIG004, from bp 2505475-2506017 (E-value 9.71e-16) and bp 2582212-2582520 (E-value 2.30e-13), which corresponded to predicted mRNA fungus_09812 and fun- 
gus_07605 respectively. HD2 protein was found on TIG004, from bp 2506752-2507018 (E-value 3.54e-05) and bp 2583597-2583803 (E-value 3.58e-04), which corresponded to predicted mRNA fungus_09811 and fungus_07606 respectively. A homologue of the Ste3 pheromone receptor was found on TIG123 (LG4) two times, which corresponded to predicted mRNA fungus_5504 and fungus_5506.

Table $2 \mid$ Summary statistics of the strictly filtered linkage map (left) and the forced order linkage map (right).

\begin{tabular}{llllll}
\hline \multicolumn{5}{l}{ Linkage map with strictly filtered data } \\
\hline Linkage Group & $\begin{array}{l}\text { No. of } \\
\text { markers }\end{array}$ & $\begin{array}{l}\text { Genetic } \\
\text { length } \\
\text { (cM) }\end{array}$ & $\begin{array}{l}\text { Longest } \\
\text { interval } \\
\text { (cM) }\end{array}$ & $\begin{array}{l}\text { Physical } \\
\text { length } \\
\text { scaffolds } \\
\text { (bp) }\end{array}$ & $\begin{array}{l}\text { Physical } \\
\text { length } \\
\text { between } \\
\text { markers }\end{array}$ \\
\hline LG1 & 117 & 66.9 & 19.7 & 3979277 & 2479225 \\
\hline LG2 & 154 & 149.8 & 34.6 & 8022737 & 7077178 \\
\hline LG3 & 74 & 41.4 & 14.6 & 4768015 & 4564448 \\
\hline LG4 & 99 & 117.2 & 35.7 & 3969078 & 3324833 \\
\hline LG5 & 131 & 67.6 & 24.0 & 3840311 & 2691605 \\
\hline LG11 & 64 & 12 & 6.4 & 1960525 & 1884415 \\
\hline LG6 & 157 & 142.3 & 28.4 & 5767440 & 5748446 \\
\hline LG7 & 29 & 39.5 & 18.7 & 4857727 & 1621542 \\
\hline LG8 & 93 & 77.9 & 24.9 & 1554905 & 2950698 \\
\hline LG9 & 174 & 126.9 & 24.8 & 5319968 & 4810179 \\
\hline LG10 & 38 & 15.4 & 7.3 & 4220926 & 1015891 \\
\hline LG12 & 210 & 140.1 & 22.5 & 7803601 & 7487537 \\
\hline LG13 & 77 & 104.3 & 23.8 & 5385040 & 4314011 \\
\hline
\end{tabular}

$\begin{array}{lllll}\text { Total: } & 1417 & 1101.3 & 61449550 & 49970008\end{array}$

Physical/

Genetic dis-

tance ratio:
$17.9 \mathrm{cM} / 22.0 \mathrm{cM} /$

$\mathrm{Mb} \quad \mathrm{Mb}$ 


\section{Forced order Linkage map}

\begin{tabular}{|c|c|c|c|c|c|c|c|}
\hline Linkage Group & $\begin{array}{l}\text { No. } \\
\text { of } \\
\text { mark- } \\
\text { ers }\end{array}$ & $\begin{array}{l}\text { Ge- } \\
\text { netic } \\
\text { length } \\
\text { (cM) }\end{array}$ & $\begin{array}{l}\text { Lon- } \\
\text { gest } \\
\text { inter- } \\
\text { val } \\
(\mathrm{cM})\end{array}$ & $\begin{array}{l}\text { Physical } \\
\text { length } \\
\text { scaffolds } \\
\text { (bp) }\end{array}$ & $\begin{array}{l}\text { Physical } \\
\text { length } \\
\text { between } \\
\text { markers } \\
\text { (bp) }\end{array}$ & $\begin{array}{l}5 \text { ' putative } \\
\text { telomere }\end{array}$ & $\begin{array}{l}3^{\prime} \\
\text { puta- } \\
\text { tive } \\
\text { telo- } \\
\text { mere }\end{array}$ \\
\hline LG1 & 167 & 71.6 & 18.2 & 3979277 & 3352014 & Yes & Yes \\
\hline LG2 & 217 & 156.8 & 21.6 & 8022737 & 7077178 & No & Yes \\
\hline LG3b & 195 & 122.7 & 43.2 & 4155742 & 4069311 & No & No \\
\hline LG4 & 135 & 95.5 & 22.7 & 3969078 & 3408123 & No & No \\
\hline LG5_LG11 & 241 & 98.9 & 35.2 & 5800836 & 5244314 & No & No \\
\hline LG6 & 254 & 128.4 & 22.7 & 5767440 & 5748446 & Yes & No \\
\hline LG7_LG8 & 152 & 139.8 & 21.6 & 6412632 & 5519496 & Ribosome & Yes \\
\hline LG9 & 202 & 108.0 & 18.2 & 5319968 & 4889355 & Yes & No \\
\hline LG10_TIG058 & 154 & 89.8 & 18.2 & 4647489 & 4272535 & Yes & No \\
\hline LG12 & 234 & 130.7 & 19.3 & 7803601 & 7487537 & No & Yes \\
\hline LG13 & 119 & 121.6 & 34.1 & 5385040 & 4678457 & Yes & Yes \\
\hline LG14 & 96 & 62.5 & 18.2 & 3383919 & 2987205 & No & No \\
\hline LG15 & 63 & 44.3 & 18.2 & 2005147 & 1697156 & No & Yes \\
\hline Total: & 2229 & 1370.4 & & 66652906 & 60431127 & & \\
\hline $\begin{array}{l}\text { Physical/ } \\
\text { Genetic dis- } \\
\text { tance ratio: }\end{array}$ & & & & $\begin{array}{l}20.6 \mathrm{cM} / \\
\mathrm{Mb}\end{array}$ & $\begin{array}{l}22.7 \mathrm{cM} / \\
\mathrm{Mb}\end{array}$ & & \\
\hline
\end{tabular}



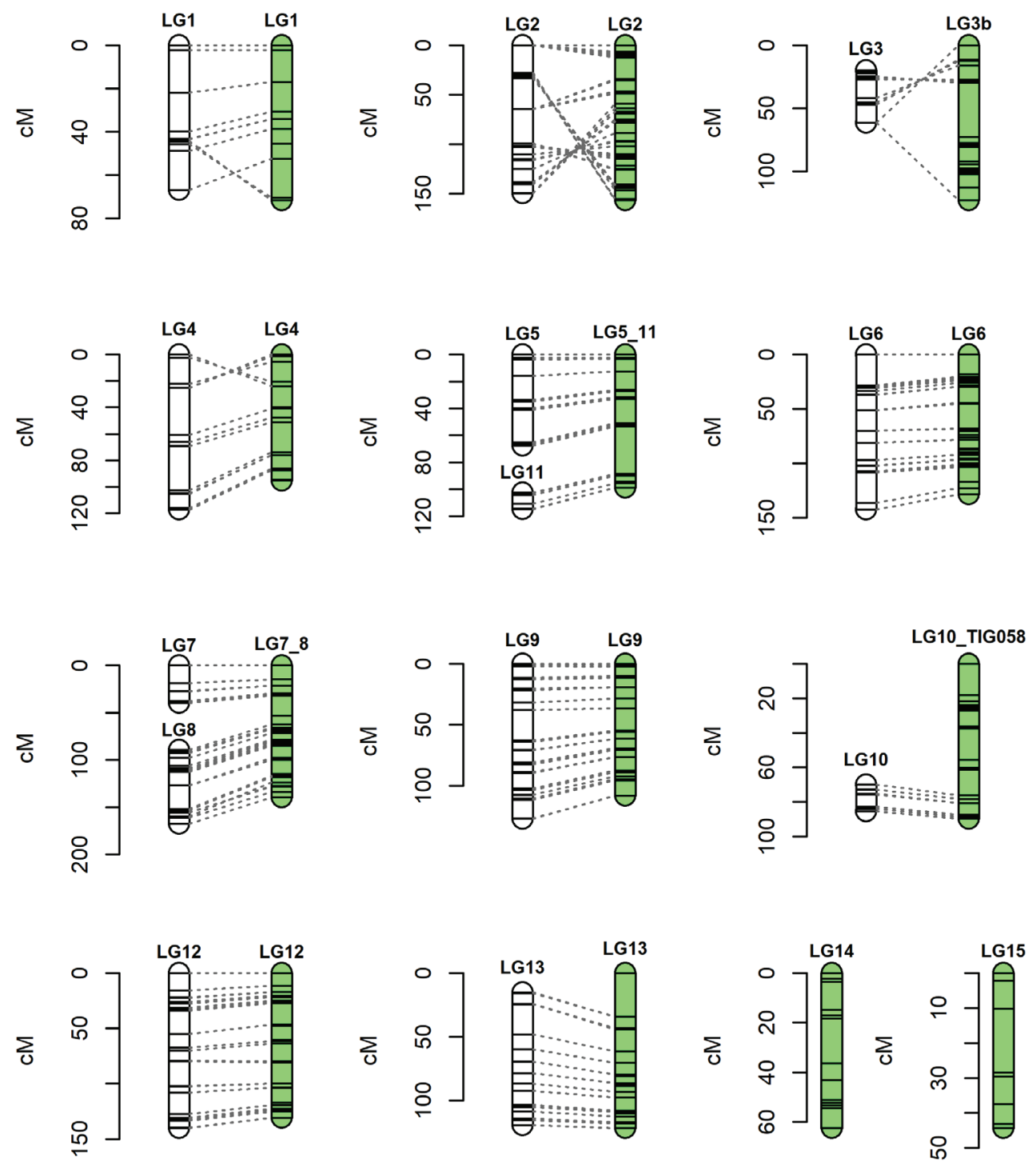

Figure 4 | Comparison between linkage map (white) and forced order linkage map(green). Lines indicate connection between identical markers.

\section{Forced order Linkage map and Recombination Frequency}

Our second mapping approach of calculating linkage between distal markers of the contigs in our v2.0 assembly and subsequently calculating the recombination frequency between markers, yielded a map with a total map length of $1370.4 \mathrm{cM}$ (Table 2, Supplementary Figure 4, Supplementary Table 4 and 5). We could include 30 contigs from the v2.0 assembly, three more than in the first mapping approach, adding up to 66.7 
$\mathrm{Mb}$, which is $95.2 \%$ of the total v2.0 assembly. Furthermore, the total physical length between markers increased to $60.4 \mathrm{Mb}$, which is $86.3 \%$ of the total v2.0 assembly. Comparison of both mapping approaches (Figure 4 and Table 2) showed that for LG1, LG2, LG4, LG6, LG9 and LG12 the longest genetic interval between two markers decreased. Also, for LG4, LG6, LG9 and LG12 the total map length decreased, even though the added physical length between markers stayed the same or increased. For LG1 the map length increased by 7\% whereas the added physical length between markers increased by $35 \%$. The map length of LG2 increased by $5 \%$, but the added physical length between markers stayed the same. For the combined linkage groups, LG5_LG11 and LG7_LG8, map length increased by $15 \%$ and $21 \%$, whereas added physical length between markers increased by $24 \%$ and 19\% respectively. For LG10, LG14 and LG15 it is not possible to make a good comparison and they were (largely) not present in the strictly filtered linkage map. Finally, for LG3 the genetic map length increased by almost $200 \%$, yet the added physical length between markers decreased by $11 \%$.

In a linkage map with many more markers than individuals it is expected that there are also many markers that are identical between individuals, i.e. that show exactly the same segregation pattern. There is, however, also the possibility that these larger regions without segregation are the result of uneven recombination across the genome. To examine the possibility of uneven recombination rates, we plotted the genetic distances against physical distances (Figure 5). The plots reveal that for some contigs the genetic position increased in jumps rather than in a continuous fashion, e.g. in LG1 and LG13. To test whether outlier genetic distances in our genetic map deviate from an even distribution across the genome we compared the observed number of crossovers between two markers to a set of randomly sampled numbers of crossovers between those markers, under the assumption that physical marker distance is correlated to number of crossovers. This showed that there are several intervals that have significantly more or less recombination than others in all linkage groups (Figure 1). We found five "cold intervals" with less recombination than expected and 48 "hot intervals" with more recombination than expected. The same test, but for grouped regions of approximately $500000 \mathrm{bp}$, found 39 "cold regions" and 25 "hot regions. When we compared the outcomes of the grouped and non-grouped marker distances, 28 "hot regions" were present in both results and four "cold regions" (Supplementary Table 6). 
LG1

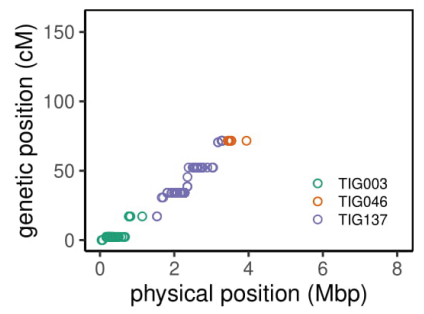

LG4

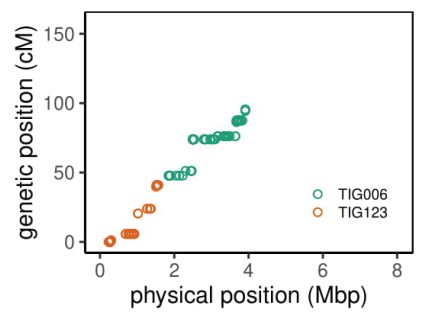

LG7_LG8

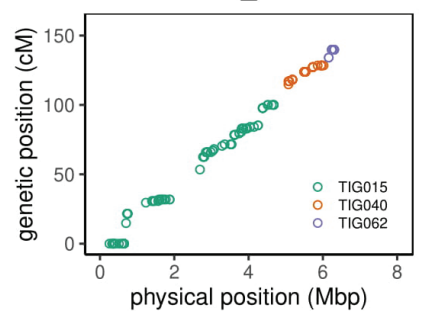

LG12

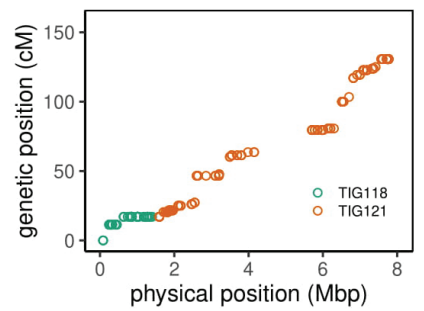

LG15

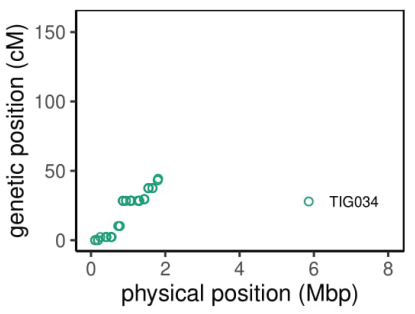

LG2

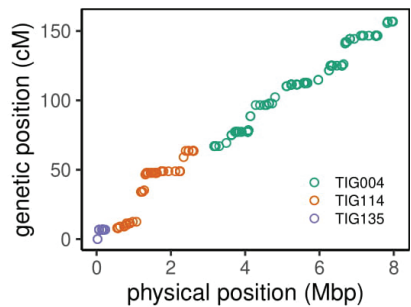

LG5_LG11

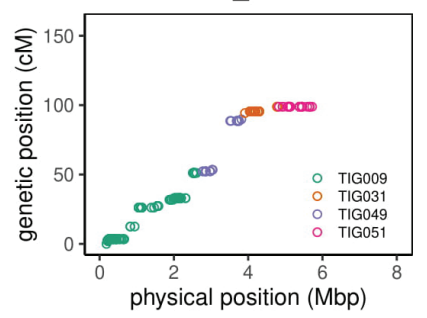

LG9

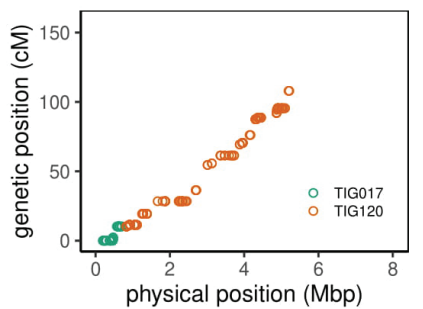

LG13

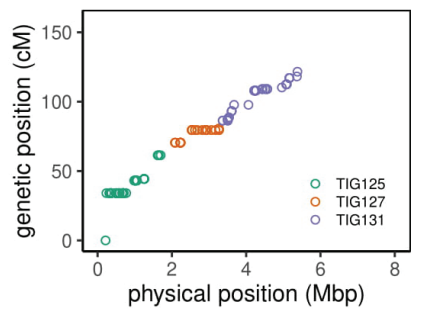

physical position (Mbp)

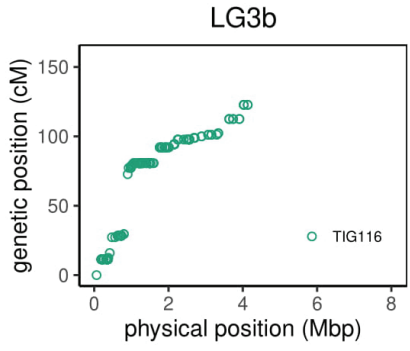

LG6

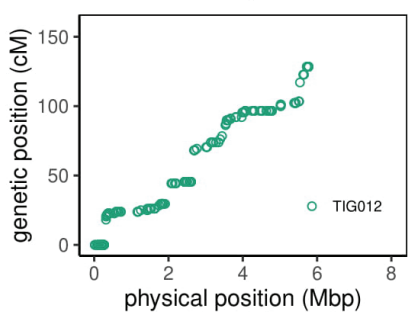

LG10_TIG058

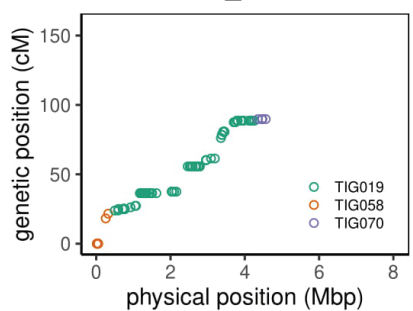

LG14

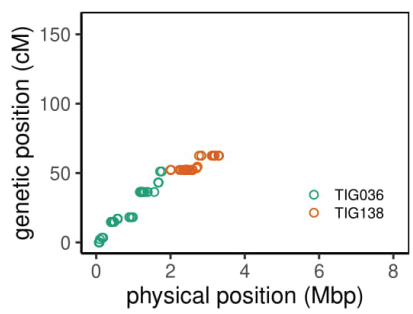

Figure 5 Plots of each linkage group in the forced-order linkage map with physical position of a marker as mapped to our assembly and on the $y$-axis the genetic position on the forced-order linkage map. 


\section{Discussion}

\section{Genome assembly}

We used long-read sequencing, the CANU assembler, and Pilon polishing with short reads to create a significantly more contiguous assembly of the genome of the Termitomyces species that is associated with $M$. natalensis. The high BUSCO score of the v1.0 genome showed that it was indeed assembled well for unique regions of the genome. As the BUSCO score of our v2.0 assembly even showed a slight improvement compared to the v1.0 assembly, improved contiguity of the genome did not come at the cost of the accuracy of single gene assembly. Although the lift-over of the annotation from the v1.0 assembly to the v2.0 assembly provides a rough annotation of the genome, this is just the starting point of the annotation process. The next step would be to improve the annotation with transcriptome data and subsequently manually curate it. The main advantage of lifting over the present annotation is that it enables a translation of the results of previous studies that have used the v1.0 annotation (e.g. Poulsen et al. 2014, da Costa et al. 2018).

\section{Two mapping approaches}

One of the issues with sequencing approaches to generate SNP data for linkage mapping is marker sites with many missing calls or low coverage calls. To increase read depth per marker we used a less frequent cutter than is often otherwise used in GBS. The main drawback of this approach is that it reduces the number of SNPs that are found. When trying to use linkage mapping to connect and order a reference assembly it depends on how small the scaffolds of the assembly are whether a less frequent cutter would also include smaller scaffolds. Although we could use only $0.14 \%$ of the raw SNPs, we managed to connect almost $90 \%$ of the assembly when strictly filtering the data. This shows that for linkage mapping a less frequent cutter is suitable. For comparable future studies, bearing in mind the continuously reducing cost of sequencing, one could also consider low coverage, whole genome sequencing (Rastas 2017). The latter option is especially interesting when aiming to study the recombination landscape in more detail as it is only dependent on the presence of SNPs between the parental individuals. In contrast to GBS, low coverage whole genome sequencing is not dependent on the presence of a restriction site near the SNP in both parental genotypes. Low coverage whole genome sequencing would produce more genotyping errors, but new linkage mapping algorithms such as LM3 use information on genotype uncertainty to be able to reliably use low coverage, error prone data (Rastas 2017).

Including markers with many missing values or low coverage in a linkage map impacts the calculation of marker distance and may change marker order and distance. However, not including these markers also means throwing away markers that do give 
reliable information. In addition to missing values, markers that have a very skewed allele frequency are also usually filtered out for linkage analysis. Skewed allele frequency may indicate erroneous calls and most mapping approaches assume mendelian segregation ratios. However, in a biparental population such as ours, larger regions that show skewed segregation may be the result of segregation distortion, often the result of viability selection on element(s) in these regions (Zhan and Xu 2011).

To deal with the above described dilemma, we chose to take two mapping approaches. The first was to make a 'regular' linkage map by strictly filtering the SNP-data so they are reliable markers for genetic mapping. In the second approach we filtered less strictly on missing data and skewed allele segregation, and manually checked all markers at the end of each contig to see whether they were supported by surrounding markers and whether the alignment to the genome looked correct. Subsequently, we calculated the extent of linkage between the first two and last two markers of each remaining contig. This approach allowed us to include, in addition to the more strictly filtered dataset, four contigs with a combined length of $5.8 \mathrm{Mb}$, i.e. more than $8 \%$ of the assembly.

\section{LG3}

In general, the two mapping approaches were highly congruent for the contigs that were included in both maps (Figure 4). One of the most obvious differences between our two mapping approaches was LG3, which increased with almost $200 \%$ in genetic length, but decreased $11 \%$ in physical length. In the second mapping approach it became clear that the most distal markers of TIG021 and TIG116 were not linked to each other with a LOD score higher than 5.5. Instead the most distal marker of TIG021 was linked to the second marker of TIG116. Alignment of TIG021 against the whole reference assembly showed that the region with the markers of TIG021 that are linked to the second marker of TIG116, also aligns to the region in LG116 that follows the second marker. In other words, the linkage between TIG021 and TIG116 in our strictly filtered dataset is most likely an artefact of erroneous SNP calling due to a duplication between a region of TIG021 and TIG116.

Still, removing TIG021 does not by itself explain the large increase in map length of LG3. On closer inspection a region of $3 \mathrm{Mb}$ long was filtered out in the strictly filtered map because of a skewed minor allele frequency (cumulative p-value lower than 0.01 under a binomial distribution). This explains a change in the linkage map, yet it does not explain the relatively small interval of $0.1 \mathrm{Mb}$ with a genetic distance of $43.2 \mathrm{cM}$ just adjacent to this region.

\section{Segregation distortion}

In addition to the region on LG3 (TIG116) that has a very skewed allele frequency, we observed a significantly skewed allele frequency for more regions of the genome 
(Supplementary Table 7). This is also the reason that the contigs included in LG14 and LG15 were not present in the strictly filtered dataset. This confirms the possibility that by filtering out SNP markers with skewed allele frequencies, regions with segregation distortion are not present in a linkage map.

In this context it is also interesting to note that despite extensive testing and repetition we were never able to obtain one of the two constituent homokaryons of the parental heterokaryon, while we were able to repeatedly recover the other parental type (mt50a). This may be due to lethal recessive mutations in the not recovered parental homokaryon, making it impossible for it to grow by itself. It has previously been found for Schizophyllum commune that there is co-adaptation between the two nuclei of heterokaryons that have co-evolved during long-term vegetative growth. In a co-adapted strain of $S$. commune at least one nucleus was found with a deleterious mutation that was compensated for by a mutation in the co-evolved nucleus (Clark and Anderson 2004). It is not unlikely that a similar co-adaptation can be found in a Termitomyces heterokaryon, as these heterokaryons can grow vegetatively for multiple decades in a termite nest (Aanen et al. 2009).

\section{Mating type}

Termitomyces fungi have been shown to lack clamp connections, which are the typical structures formed by most species of basidiomycetes in dikaryons and not in monokaryons. Also, as so far it is impossible to induce mushroom formation in this species, it is hard to judge whether a mating is successful. However, Nobre et al. (2014) showed that a heterokaryon can be distinguished from a homokaryon based on phenotypic differences. Also, as we have shown by testing with an RFLP marker, we managed to separate homokaryons from heterokaryons.

Nonetheless some uncertainty remained. Therefore, it is good that we used two approaches to map the mating type. As they both pointed to the same physical location in the genome it is clear that this is the mating-type region. However, it is not yet clear what the exact genes involved are as there seem to be two HD loci, located closely together. This is not unique as in Schizophyllum commune even three sets of HD1 and HD2 loci and two additional HD1 genes were found (Ohm et al. 2010).

Our mating tests indicate that Termitomyces sp. has a bipolar mating type. It is thought that the tetrapolar mating system is the ancestral state in basidiomycetes (James et al. 2006, Coelho et al. 2017). There are two main mechanisms to become bipolar: 1.) through physical linkage of the HD locus and the pheromone receptor locus, and, 2.) through the loss of the ability to recognise self from non-self in the pheromone/pheromone receptor locus through obtaining pheromone genes that fit one's own receptor. As we find a homolog of the yeast Ste3 receptor on a different linkage group than the HD 
locus, we hypothesise that this Termitomyces species became bipolar through the second mechanism, similar to what has been found in other species (James et al. 2006, Coelho et al. 2017).

\section{Karyotype of Termitomyces}

One piece of the puzzle needed to create a (nearly) finished assembly of Termitomyces is its karyotype. As long as we do not know the karyotype of Termitomyces we cannot be completely sure that we have assembled its whole genome. In our assembly we could find 15 unique sequences with putative telomeres, which would mean that Termitomyces has at least eight chromosomes. Our forced linkage map contains 13 linkage groups, comprising $95.2 \%$ of the genome, which makes it unlikely that there are more than 13 chromosomes. So, the Termitomyces genome likely contains between 8 and 13 chromosomes. This number of chromosomes is comparable to the number of chromosomes in other Agaricales (Table 3). Once the annotation is improved and transcriptome data of multiple samples are analysed, it may be possible to point out putative centromeres in our assembly as centromeres in filamentous fungi appear to be regions of more than $20 \mathrm{~kb}$ that do not have genes and transcription and low GC content (Smith et al. 2012).

One of the approaches that is often used to study fungal karyotypes is Contour-clamped homogeneous electric field electrophoresis (CHEF). We have tested this method extensively for the Termitomyces genome but were not able to separate chromosome size bands (van Oosten 2016). In addition to technical issues, such as the ability to isolate enough DNA with intact chromosomes, it may be that some Termitomyces chromosomes are too large to be separated by CHEF. In the setup we used, chromosomes larger than 8 $\mathrm{Mb}$ will not be separated, although Orbach et al. (1988) managed to separate a chromosome that was estimated at $12.6 \mathrm{Mb}$. As we found that Termitomyces likely has 8-13 chromosomes they will on average be between 6.4 and $10.5 \mathrm{Mb}$. So, in theory for some of the Termitomyces chromosomes it should be possible to separate them with CHEF, but for others it will probably not. Another, more recent karyotyping method that has successfully been used on fungi is optical mapping (Dimalanta et al. 2004, Zhou et al. 2007, Teague et al. 2010). Together with Pacbio assemblies, optical maps can produce near-complete genome assemblies (Faino et al. 2015, Seidl et al. 2015, van Kan et al. 2017).

\section{Recombination analysis}

Under the hypothesis that an inhabitant mutualist would have reduced recombination compared to free living species (Law and Lewis 1983, Bergstrom and Lachmann 2003), the recombination rate in Termitomyces should be relatively low. We found that the average recombination rate for the Termitomyces symbiont of M. natalensis is between 17.9 $\mathrm{cM} / \mathrm{Mb}$ and $22.6 \mathrm{cM} / \mathrm{Mb}$, depending on how we calculated physical distance and recombination rate (Table 2). Stapley et al. (2017) have investigated and compared re- 
Table 3 | Chromosome number, sizes and total genome size of five fungi from the order of Agaricales.

\begin{tabular}{|c|c|c|c|c|}
\hline Species & $\begin{array}{l}\text { Chromo- } \\
\text { some no. }\end{array}$ & $\begin{array}{l}\text { Genome } \\
\text { size } \\
(\mathrm{Mb})\end{array}$ & $\begin{array}{l}\text { Genetic } \\
\text { map } \\
\text { length } \\
\text { (cM) }\end{array}$ & Reference \\
\hline $\begin{array}{l}\text { Agaricus } \\
\text { bisporus }\end{array}$ & 13 & $\begin{array}{l}34 / \\
31.0\end{array}$ & $\begin{array}{l}543.8 / \\
851 / \\
1156\end{array}$ & $\begin{array}{l}\text { (Kerrigan et al. 1993, Son- } \\
\text { nenberg et al. 1996, Fou- } \\
\text { longne-Oriol et al. 2010, Fou- } \\
\text { longne-Oriol et al. 2011) }\end{array}$ \\
\hline $\begin{array}{l}\text { Flammulina } \\
\text { velutipes }\end{array}$ & 12 & 38.6 & $?$ & (Kim et al. 2000) \\
\hline $\begin{array}{l}\text { Hypsizygus } \\
\text { marmoreus }\end{array}$ & 11 & 36.3 & ? & (Lee et al. 2014) \\
\hline $\begin{array}{l}\text { Pleurotus } \\
\text { ostreatus }\end{array}$ & 11 & 35.1 & $\begin{array}{l}1000.7 \\
/ 1061\end{array}$ & $\begin{array}{l}\text { (Larraya et al. 2000, Park et al. } \\
\text { 2006) }\end{array}$ \\
\hline Pleurotus eryngii & ? (11 LGs) & 43.8 & 837.2 & $\begin{array}{l}\text { (Okuda et al. 2012, Yang et al. } \\
\text { 2016) }\end{array}$ \\
\hline Lentinula edodes & $\begin{array}{l}\text { Between } 8 \\
(\mathrm{CHEF}) \text { and } \\
13 \text { (LGs) }\end{array}$ & 40.2 & $\begin{array}{l}1006.1 / \\
908.8 / \\
1398.4\end{array}$ & $\begin{array}{l}\text { (Terashima et al. 2002, Ka- } \\
\text { zuhiro et al. 2008, Yip et al. } \\
\text { 2012, Gong et al. 2014) }\end{array}$ \\
\hline $\begin{array}{l}\text { Schizophyllum } \\
\text { commune }\end{array}$ & 14 & 35.6 & $?$ & (Ásgeirsdóttir et al. 1994) \\
\hline $\begin{array}{l}\text { Armillaria } \\
\text { ostoyae }\end{array}$ & $\begin{array}{l}?(11 \text { puta- } \\
\text { tive) }\end{array}$ & 56.6 & 1007.5 & (Heinzelmann et al. 2020) \\
\hline $\begin{array}{l}\text { Termitomyces } \\
\text { sp. }\end{array}$ & $\begin{array}{l}\text { Between } 8 \\
\text { and } 13\end{array}$ & 83.7 & $\begin{array}{l}1101.3 / \\
1370.4\end{array}$ & $\begin{array}{l}\text { (Poulsen et al. 2014; this } \\
\text { study) }\end{array}$ \\
\hline
\end{tabular}

combination rates across eukaryotes, including fungi. They find that in fungi the mean recombination rate is $48.68 \mathrm{cM} / \mathrm{Mb}$ with a minimum of $1.4 \mathrm{cM} / \mathrm{Mb}$ and a maximum of $119.9 \mathrm{cM} / \mathrm{Mb}$, which would indeed mean that the Termitomyces species we studied has a relatively low recombination frequency. However, Stapely et al. have studied only 15 fungal species, of which four are basidiomycetes, of which two are Agaricales, the order of Termitomyces. From the order of Agaricales, not included in the study of Stapely et al., Pleurotus ostreatus was found to have a recombination rate of $28.5 \mathrm{cM} / \mathrm{Mb}$ (Larraya et al. 2000) and Lentinula edodes $18.4 \mathrm{cM} / \mathrm{Mb}$ (Terashima et al. 2002), which is more comparable to the per $\mathrm{Mb}$ recombination rate that we observed. Additionally, recombination rates are very sensitive to genotyping errors and mapping algorithms. PolymapR, 
for example, the R package that was used for the strictly filtered linkage map uses the MDSMap algorithm, which has a tendency to slightly underestimate map length (Preedy and Hackett 2016). Table 3 shows that for species where multiple linkage maps were available the total map lengths between could differ significantly. Also, recombination rate can vary largely between varieties of the same species, as is the case for Agaricus bisporus var. bisporus and Agaricus bisporus var. burnettii, accounting for the large differences in map lengths of different maps (Table 3; Callac et al. 1993, Kerrigan et al. 1993, Callac et al. 1997, Foulongne-Oriol et al. 2010, Sedaghat Telgerd 2017).

Also, we can wonder if the per $\mathrm{Mb}$ recombination rate is biologically meaningful. Firstly, recombination rate is to a certain extent linked to the number of chromosomes an organism has; for correct segregation at meiosis at least one crossover event needs to take place per pair of homologous chromosomes. Secondly, all eukaryotes have a roughly similar number of genes, whereas genome size can vary several orders of magnitude (Walbot and Petrov 2001). In this sense, only the absolute size of the genetic map is relevant when discussing recombination frequency, because it will give information about the amount of recombination between the average gene combination. When only looking at absolute map length, the recombination frequency we observed in Termitomyces sp. is comparable to that of other Agaricales (Table 3).

In conclusion, the Termitomyces species we studied does seem to have a relatively low per $\mathrm{Mb}$ recombination rate compared to other fungi. However, the absolute map length is comparable to other Agaricales. Also, the Agaricales that we could compare our results to, are not necessarily free-living relatives of Termitomyces fungi. The species of which a linkage map has been constructed are all of economic importance, because they are grown for human consumption. They have been domesticated and are as such like symbionts, although the symbiosis is of a very recent origin compared to the termite symbiosis with termites. To come to a more substantiated conclusion about the recombination rate it would be interesting to determine the meiotic recombination rates in more Termitomyces species as well as in more closely related free-living relatives from the family of Lyophyllaceae.

As Stapley et al. (2017) point out, understanding how and why recombination rate varies between taxa, species, sexes, individuals, and across the genome is a major challenge in biology. Also, technically, recombination rates are very sensitive to genotyping errors and mapping algorithms, so it is hard to compare map lengths between different studies. How recombination landscapes evolve and what impact this has on the sexually reproducing organisms has not received much attention. However, as argued by Law and Lewis (1983) and Bergstrom and Lachmann (2003), selection can act on the evolution of recombination landscapes and may have important implications for the stability of mutualisms. We showed that in the Termitomyces symbiont of M. natalensis regions with 
high recombination rates, as well as low recombination rates were present. It is not surprising to find differences in recombination rates across the genome; so-called hotspots have been observed in many species (Stapley et al. 2017). However, it has also been shown that the preferential placement of crossovers may vary greatly under different conditions (Cotton et al. 2009, Zhang et al. 2017, Lloyd et al. 2018). So future studies of the recombination rates of Termitomyces species would greatly benefit from analysis of multiple mapping populations obtained under different conditions. Yet, as currently it is impossible to obtain induced Termitomyces mushrooms in a laboratory environment, this would require extensive fieldwork.

From a technical perspective, it is important to note that in assessing the recombination landscape of the presently studied Termitomyces we assumed that the physical genome of the parents of our mapping population was identical to the individual that was sequenced for the reference assembly. In other words, we assumed that the physical position of the SNP markers was identical. On the whole this is a reasonable assumption as 1.) both Termitomyces isolates were taken from the same termite species, M. natalensis, which was shown to only cultivate a single biological species of Termitomyces (De Fine Licht et al. 2006, Aanen et al. 2007, Nobre et al. 2014), and, 2.) both isolates were taken from the same sampling location, which means that they are likely to come from an interbreeding population. To visualise the structural similarity between the reference assembly and our mapping population we assembled the Illumina reads from mt50a, aligned the assemblies and visualised the alignment using dotPlotly (Supplementary Figure 5). Even though this alignment would probably not show large inversions, because the assembly of $\mathrm{mt50a}$ is very fragmented, it did indicate that overall the assemblies were structurally the same. However, important differences can be observed that also influence our assessment of some regions of high and low recombination. For example, the alignment shows that on LG12, TIG121 there is a region that is present in the reference assembly, but not in mt50a (Supplementary Figure 6). This region coincides with a $1.5 \mathrm{Mb}$ region without any markers, which means that the recombination analysis in this region is not reliable. Including more individuals in our mapping population as well as making a more contiguous assembly of $\mathrm{mt} 50$ a would help improve the analysis of high and low recombination regions.

\section{Acknowledgements}

We would like to acknowledge Linda van Oosten for her extensive experimentation with the CHEF setup, Ben Auxier for discussion, and reading parts of the manuscript, Fons Debets, Erik Wijnker and Hans de Jong for discussion and Alexander Tulloch Lapresa for providing me with his interesting proposal entitled: "Hedging your bets: How fungi may adapt crossover positioning to favour specific environmental adaptation". 
This work was funded by the Dutch Research Council (NWO) (S.M.E.V.: ALW Open competition 824.01.002, D.K.A. VICI NWO 86514007). The work of P.M.B. was partly funded through the TKI polyploids project (BO-26.03-009-004 \& BO-50-002-022) 


\section{Supplementary material}
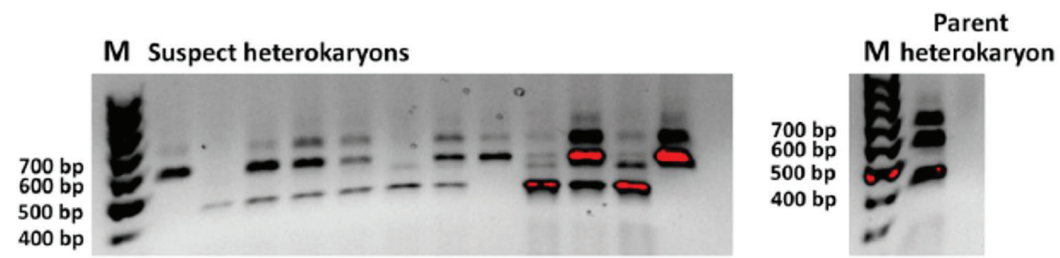

\section{Presumed homokaryons}

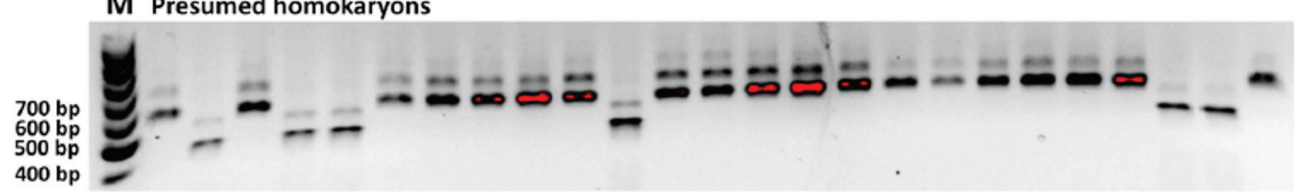

Supplementary Figure 1| Heterozygosity test 37 individuals from the mapping population. PCR-RFLP analysis was performed on 12 suspect heterokaryons (top left) and 25 presumed homokaryons (bottom), using a marker for which the parent heterokaryon (top right) was heterozygous. Sizes of the 100 base pair (bp) ladder (lane M) are indicated on the left. The length of the undigested fragment targeted by PCR was $591 \mathrm{bp}$ and digestion products of $417 \mathrm{bp}$ and 173 bp were expected for one of the two alleles.
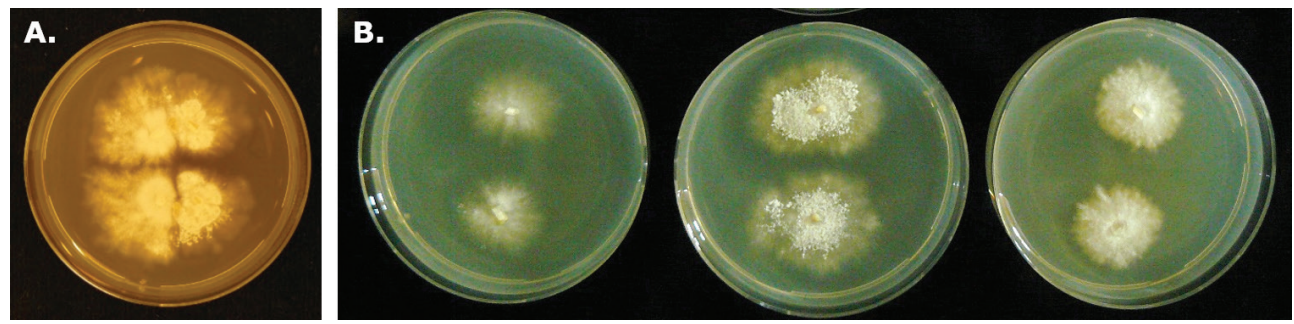

Supplementary Figure 2| Example of mating type assessment. Figure A. shows a duplicate cross between individual 3 and 7. Figure B. shows a further clarification by transferring a piece of mycelium from both homokaryons (left and right) and the interaction zone of the cross (middle). 


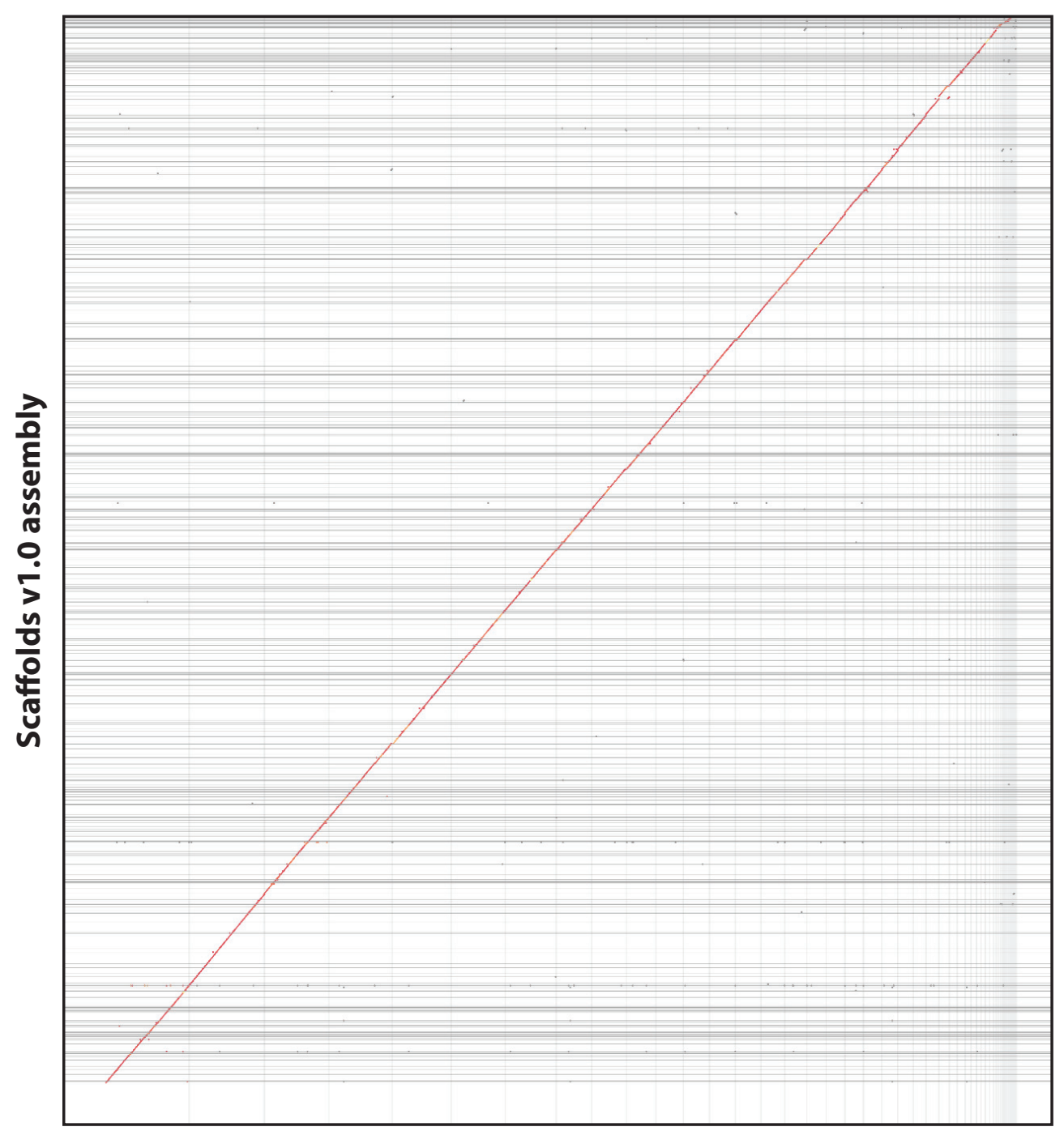

\section{Contigs v2.0 assembly}

Supplementary Figure $3 \mid$ DotPlotly visualisation of the alignment between the Termitomyces assembly v2.0 and the Termitomyces v1.0 assembly. On the x-axis the contigs of the v2.0 assembly, on the $y$-axis the scaffolds of the v1.0 assembly. 

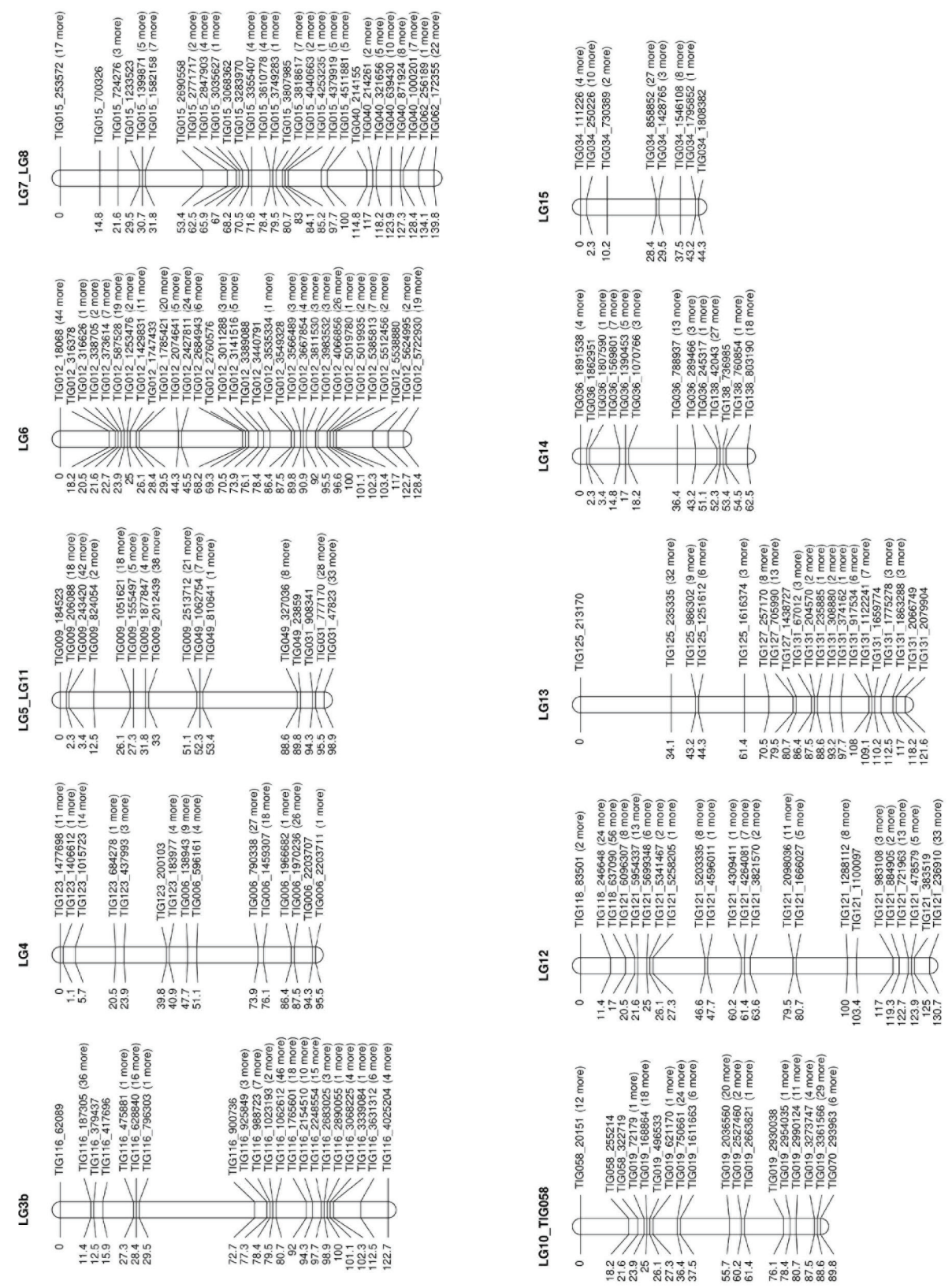

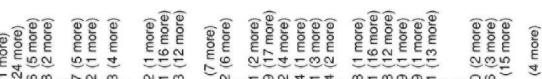

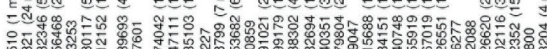

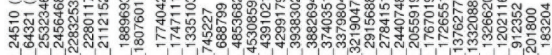

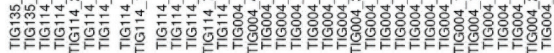

ษ
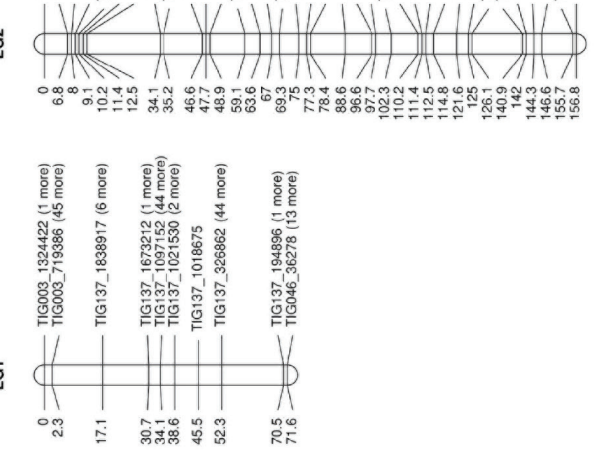

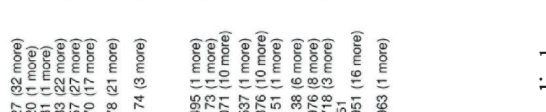

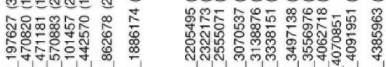

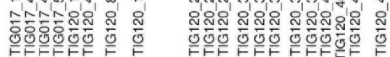

乌

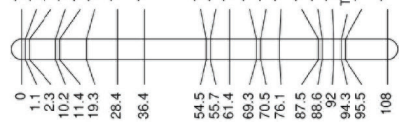

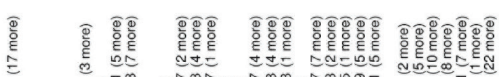

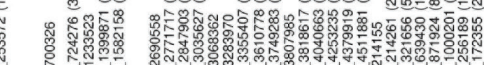

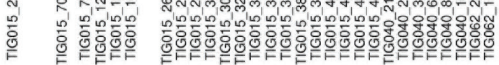

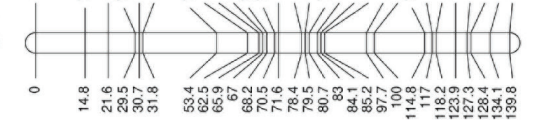




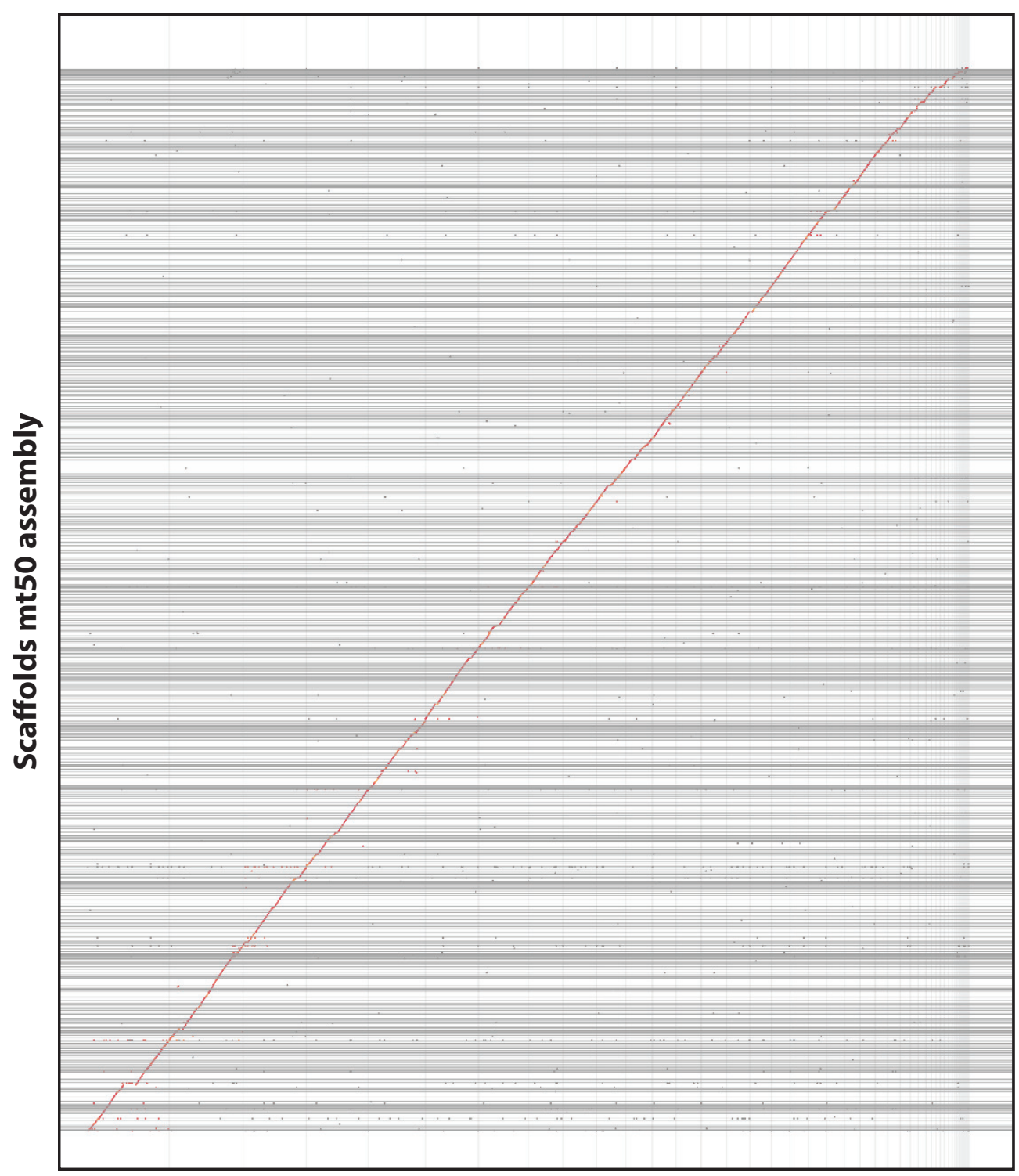

\section{Contigs v2.0 assembly}

Supplementary Figure 5| DotPlotly visualisation of the alignment between the v2.0 reference assembly and the assembly of $\mathrm{mt} 50 \mathrm{a}$. On the $\mathrm{x}$-axis the contigs of the v2.0 reference genome, on the $y$-axis the scaffolds of the mt50a assembly. 


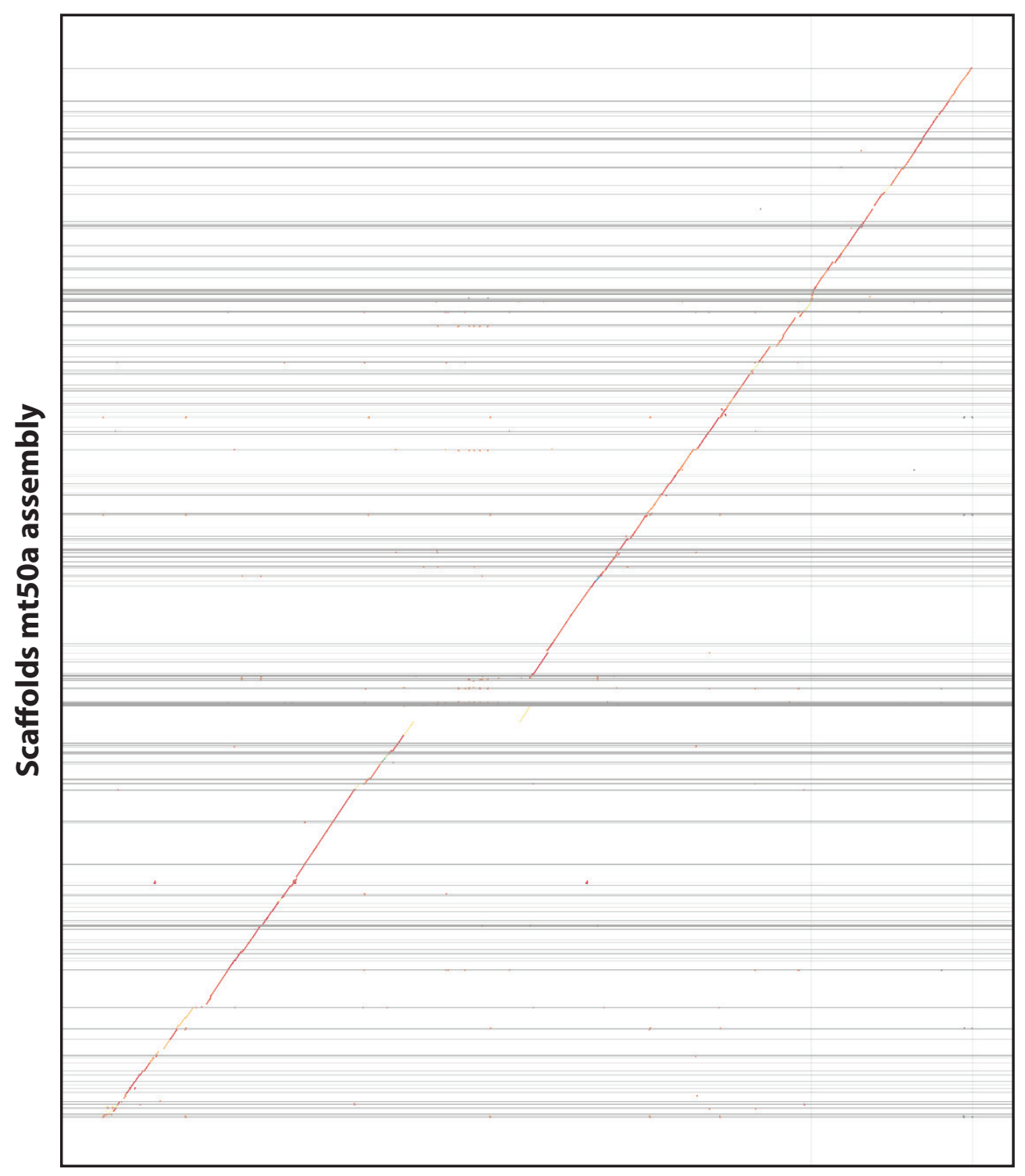

\section{LG12 Contigs v2.0 assembly}

Supplementary Figure 6 | DotPlotly visualisation of the alignment between the contigs of the v2.0 reference assembly that form LG12 in the forced order linkage map and the assembly of $\mathrm{mt50a}$. On the x-axis the contigs of the new reference genome, on the y-axis the scaffolds of the mt50a assembly. 
Supplementary Table 1| Crossing table between 30 individuals from the mapping population against 6 individuals from the mapping population with legend. Shows that this Termitomyces species likely has a bipolar mating type.

\begin{tabular}{|l|l|}
\hline & Not setup \\
\hline & Successful cross \\
\hline & No cross \\
\hline & No clear outcome \\
\hline
\end{tabular}

\begin{tabular}{|c|c|c|c|c|c|c|}
\hline & 3 & 6 & 19 & 32 & 49 & 60 \\
\hline \multicolumn{7}{|l|}{3} \\
\hline \multicolumn{7}{|l|}{6} \\
\hline \multicolumn{7}{|l|}{19} \\
\hline \multicolumn{7}{|l|}{32} \\
\hline \multicolumn{7}{|c|}{49} \\
\hline \multicolumn{7}{|c|}{60} \\
\hline \multicolumn{7}{|c|}{7} \\
\hline 8 & $\mathrm{NA}$ & & & & & \\
\hline \multicolumn{7}{|l|}{12} \\
\hline \multicolumn{7}{|c|}{14} \\
\hline \multicolumn{7}{|l|}{15} \\
\hline \multicolumn{7}{|l|}{20} \\
\hline \multicolumn{7}{|c|}{21} \\
\hline \multicolumn{7}{|l|}{22} \\
\hline \multicolumn{7}{|c|}{23} \\
\hline \multicolumn{7}{|c|}{24} \\
\hline \multicolumn{7}{|l|}{26} \\
\hline \multicolumn{7}{|c|}{29} \\
\hline \multicolumn{7}{|l|}{31} \\
\hline \multicolumn{7}{|c|}{34} \\
\hline \multicolumn{7}{|c|}{35} \\
\hline \multicolumn{7}{|l|}{36} \\
\hline \multicolumn{7}{|c|}{38} \\
\hline \multicolumn{7}{|l|}{42} \\
\hline \multicolumn{7}{|c|}{43} \\
\hline \multicolumn{7}{|c|}{47} \\
\hline \multicolumn{7}{|l|}{48} \\
\hline \multicolumn{7}{|c|}{51} \\
\hline \multicolumn{7}{|c|}{55} \\
\hline 58 & & & & & & \\
\hline
\end{tabular}




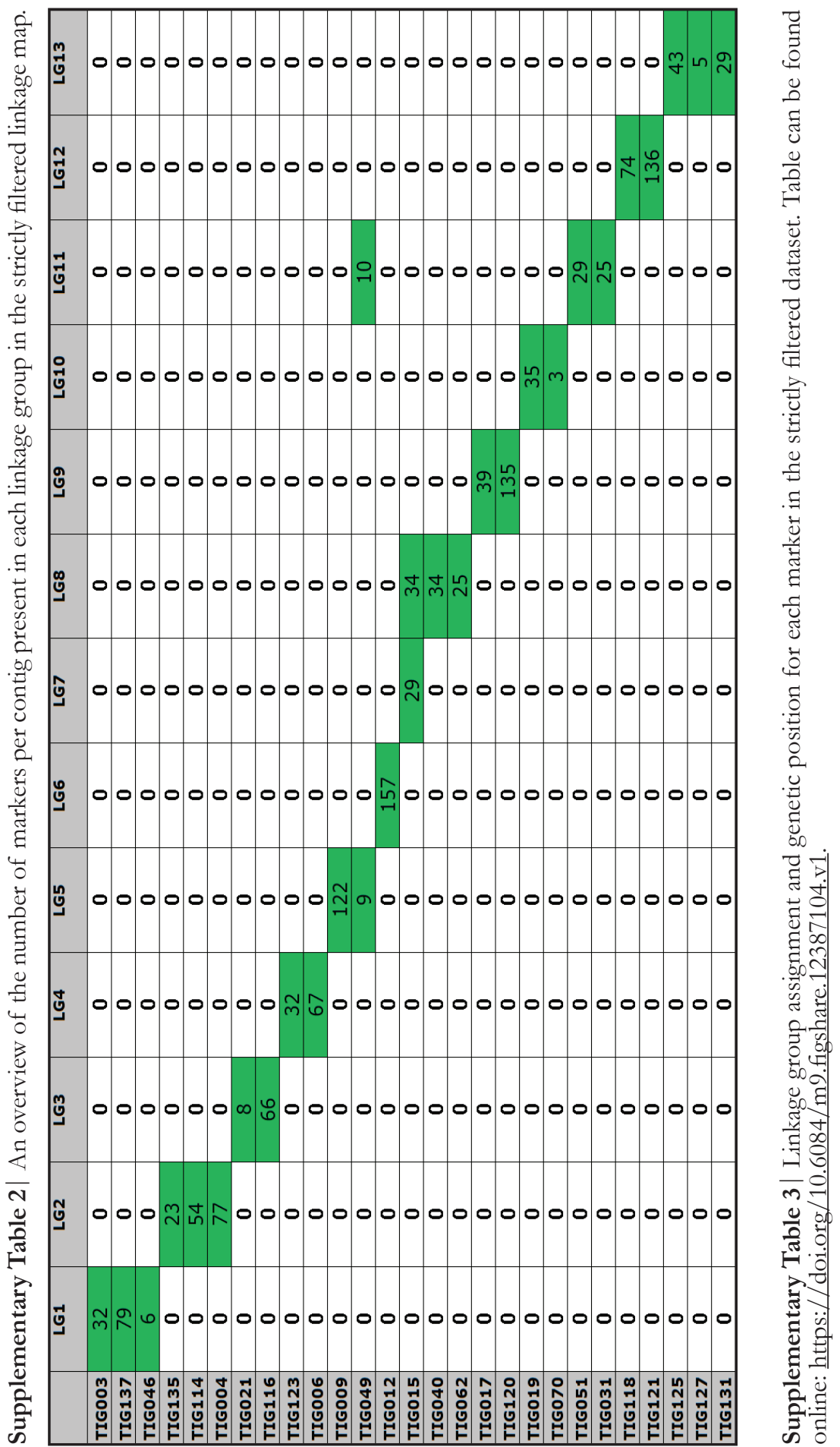




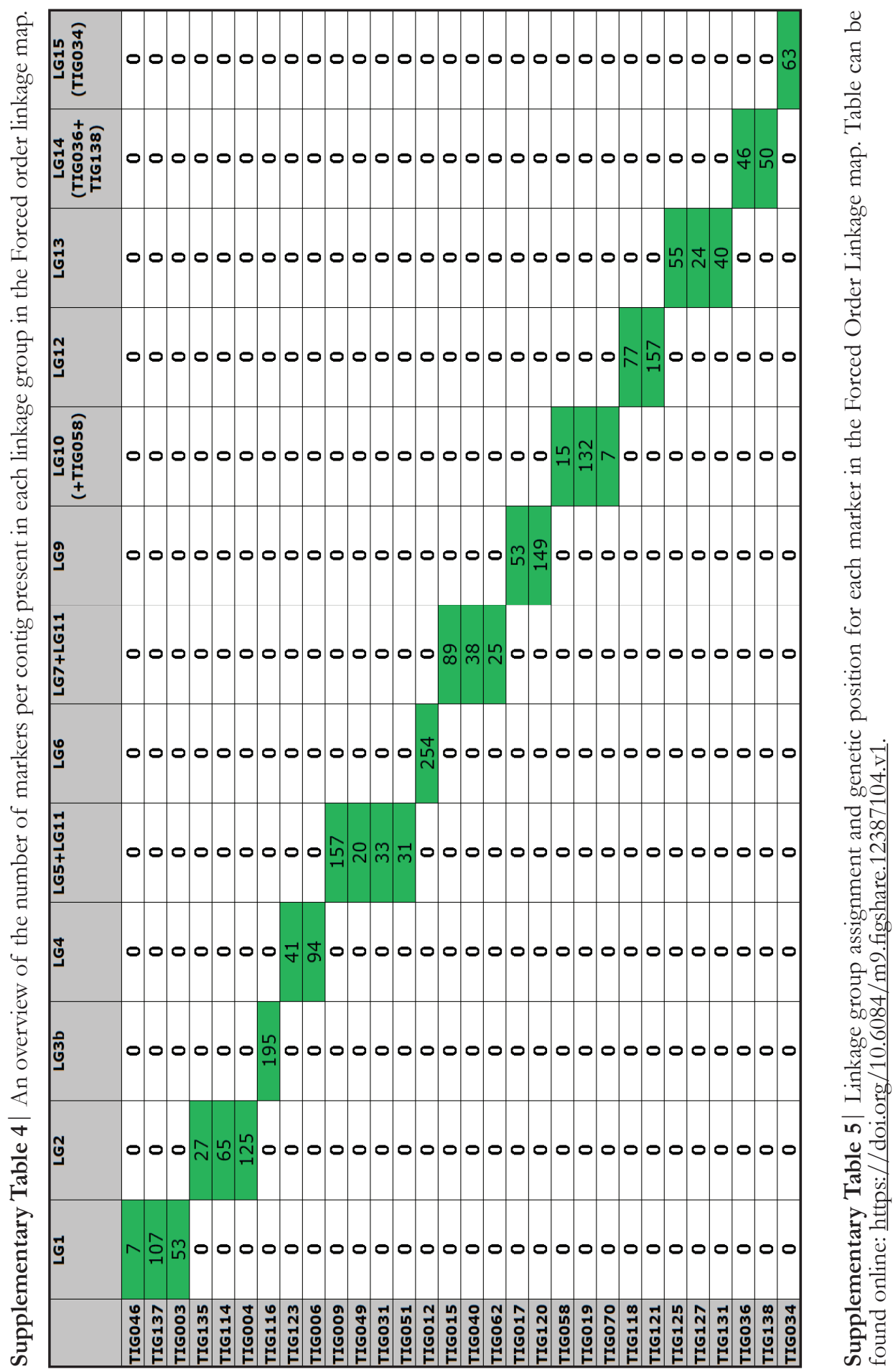


Supplementary Table $6 \mid$ Intervals between markers and between grouped markers with less (cold) or more (hot) recombination than expected. Table can be found online: https://doi. org $/ 10.6084 / \mathrm{m} 9$. figshare.12387104.v1.

Supplementary Table $7 \mid$ Markers with a skewed minor allele frequency (cumulative p-value $<0.05$ under binomial distribution). Table can be found online: https://doi.org $/ 10.6084 / \mathrm{m}$ ) figshare.12387104.v1. 


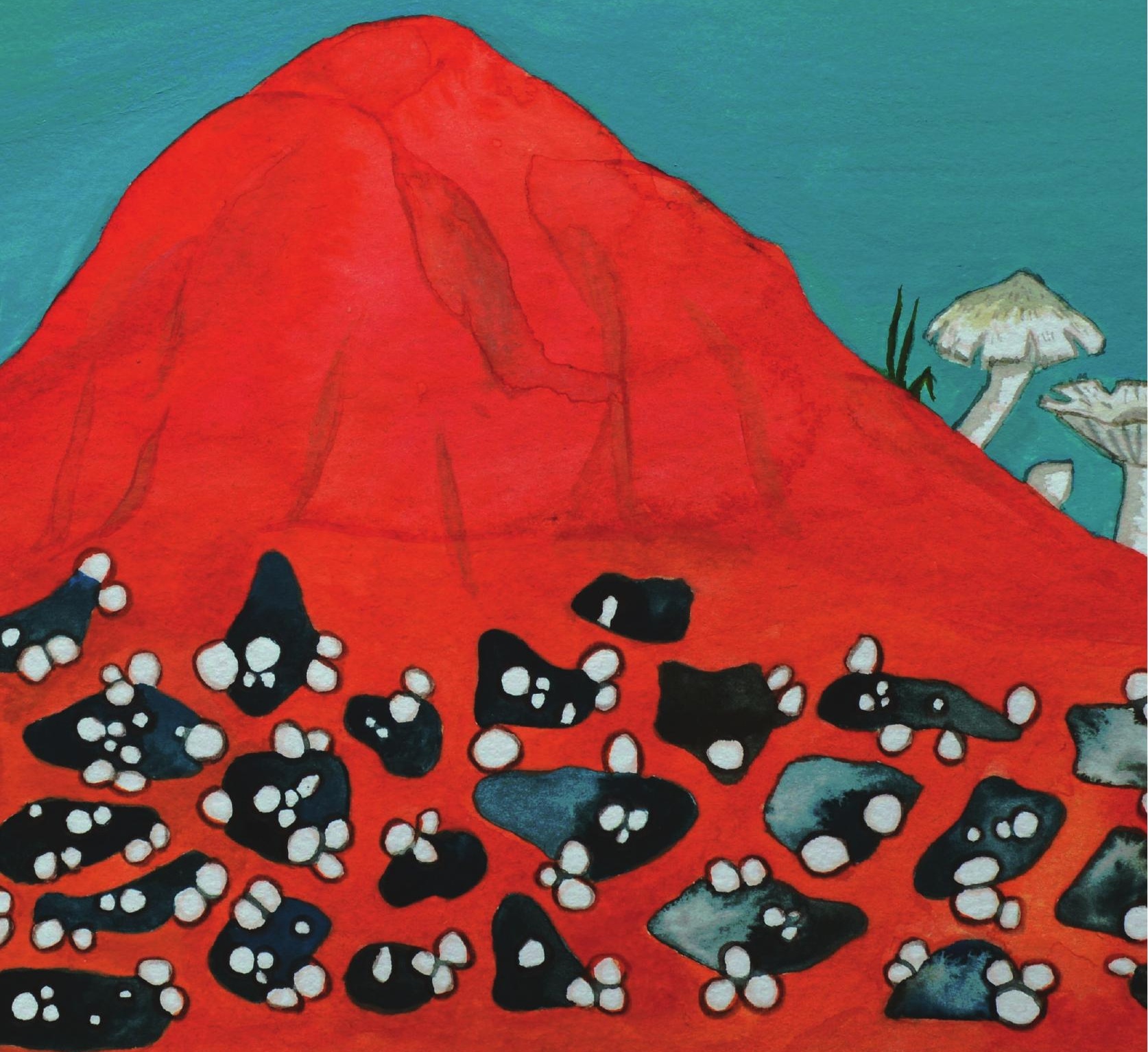




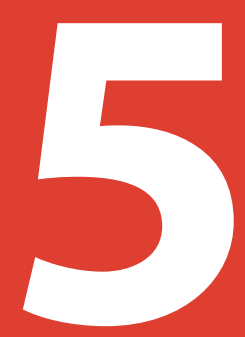

\title{
Unholy marriages and eternal triangles - how competition in the mushroom life cycle can lead to genomic conflict
}

\author{
Sabine M.E. Vreeburg, \\ Kristiina Nygren \& Duur K. Aanen \\ Published in Philosophical \\ Transactions B
}




\section{Abstract}

In the vast majority of sexual life cycles, fusion between single-celled gametes is directly followed by nuclear fusion, leading to a diploid zygote and a life-long commitment between two haploid genomes. Mushroom-forming basidiomycetes differ in two key respects. First, the multicellular haploid mating partners are fertilised in their entirety, each cell being a gamete that simultaneously can behave as a female, i.e. contributing the cytoplasm to a zygote by accepting nuclei, and male gamete, i.e. only donating nuclei to the zygote. Second, after gamete union the two haploid genomes remain separate so that the main vegetative stage, the dikaryon, has two haploid nuclei per cell. Only when the dikaryon produces mushrooms, the nuclei fuse to enter a short diploid stage, immediately followed by meiosis and haploid-spore formation. So in basidiomycetes, gamete fusion and genome mixing (sex) are separated in time. The 'living apart together' of nuclei in the dikaryon maintains some autonomy for nuclei to engage in a relationship with a different nucleus. We show that competition among the two nuclei of the dikaryon for such 'extramarital affairs' may lead to genomic conflict by favouring genes beneficial at the level of the nucleus, but deleterious at that of the dikaryon. 


\section{Introduction}

Sex (see Box 1 for our definition of sex) starts with fusion between gametes, which brings the genomes of different organisms together in a single zygote and thereby introduces competition between homologous genes for transmission to offspring. Furthermore, during sexual reproduction, an individual transmits its genome not as a single entity but as different fractions, i.e. different sets of genes that replicate together (referred to as co-replicons by Cosmides and Tooby (1981)), which can be subject to different transmission rules. For example, in organisms with genetic sex determination, sex chromosomes are unevenly transferred to males and females, in contrast to the autosomes. Similarly, cytoplasmic genes, such as mitochondrial or chloroplast genomes, usually are passed on via the female lineage only. These different transmission rules imply that different co-replicons within an individual can have different fitness optima, leading to conflict between them. So, in this respect, sex has two consequences: i) it introduces competition between homologues within a single co-replicon category and ii) it introduces conflict between the genes of different co-replicons due to different transmission rules. It is important here to emphasise the difference between competition and conflict. Competition occurs between entities within the same category, such as between homologous genes, or between different mitochondrial genomes. Essentially, competition implies: wanting the same. In contrast, there can only be conflict between entities of different categories within an individual, such as between the nuclear and mitochondrial genomes, or between non-homologous genes within a nuclear genome. Essentially, therefore, conflict means: wanting something different, such as 'meiotic drive' versus 'fair meiosis', or 'male sterility' versus 'male fertility'.

Both consequences of sex stated previously provide opportunities for selfish (or 'ultra-selfish' (Crow 1988)) genes, i.e. genes that decrease the fitness of the individual carrying them and therefore depend on other means to increase in frequency, to compensate for the harm they incur on their host. Such compensation can occur through horizontal spread to other individuals or through violation of a transmission 'rule' such as meiosis, leading to meiotic drive. Ultimately, ultra-selfish genes can be selected due to intra-individual competition between homologous genes. Since ultra-selfish genes per definition decrease individual fitness, they will be in conflict with all other unlinked genes in the genome, and will thus lead to genomic conflict (Hurst et al. 1996).

Sexually reproducing organisms have evolved various life-cycle adaptations to reduce the selective scope for ultra-selfish genes. For example, uniparental transmission of cytoplasmic genes reduces the selective scope for ultra-selfish mitochondrial and chloroplast genes, as the fates of the cytoplasmic genes and the maternal lineage become united (Cosmides and Tooby 1981, Hoekstra 1990). Also, the union of the two haploid 
nuclei into a single diploid nucleus, followed by mitotic divisions of that nucleus, and a 'fair' meiosis in the sexual organs, removes the opportunity for the haploid nuclear components to pursue their own selfish interests by, for example, outcompeting the other by faster replication. Yet, certain sexually reproducing organism groups are lacking some of these adaptations to reduce the selective scope for ultra-selfish genes. This begs the question if such organisms are more prone to genomic conflicts due to ultra-selfish genes. In this paper, we consider this question for filamentous basidiomycete fungi in which we can distinguish two categories of co-replicons based on their transmission during mating, vir. nuclei and mitochondria. (In this review, we assume that meiosis is 'fair', so we do not consider the possibility of meiotic drive, which has been found to play a role in some ascomycete fungi (Turner and Perkins 1991)).

\section{Box 1: The definition of sex}

There are many (evolutionary) biology or genetics textbooks in which sex is discussed, but no explicit definition of sex is given; it is apparently generally assumed that the reader knows what sex is. However, the implicit definitions of sex tend to be narrow and not applicable to all classes of organisms e.g. involving the fusion of two different gametes like an egg and a sperm cell. As in the example, characteristics of a species' life cycle are often implicitly included in the definition, thereby neglecting other species that are considered to have sex but have different life cycles. This caveat was also pointed out by Dick (1987), who consequently defined sexual reproduction as "the union of two haploid nuclei each derived from one of two different meioses". The advantage of Dick's definition of sex is that it allows for separation of the universal nuclear events, i.e. the effect from sex on the genome, from the typical life cycle events that can differ extensively between classes of organisms. We do think, however, that Dick's definition is incomplete in one aspect, namely the subsequent reduction of fusion product's genome by meiosis. Hence, our definition of sex is: the union of two haploid nuclei, each produced by meiosis, in due course followed by a reduction of the genome through meiosis. According to this definition, sex in basidiomycetes is separated in time from gamete fusion, which is in contrast to almost all other sexual life cycles.

Filamentous basidiomycete fungi differ in several key aspects from the most well-known sexual organisms, such as animals, plants and ascomycete fungi (Figure 1). First, gamete fusion does not occur during the single-celled stage, but between monokaryons (all terms printed in bold are explained in the legend of Figure 1), multicellular haploid mycelia, which mate in a hermaphroditic fashion. Two cells of the different monokaryons 
fuse creating one cell where both nuclei and cytoplasms are shared. Subsequently, if the two nuclei are compatible, i.e. if they have different mating types, both monokaryons allow their partner's nuclei to migrate through their mycelium until they reach the other side of the former monokaryon (now dikaryon) (Figure 1). Thus, barring the narrow zone of interaction where the cells fuse, there is no exchange of mitochondria. Second, unlike the vast majority of sexual organisms, upon fertilisation, the nuclei do not fuse, but remain separate in each cell of the dikaryon. It is only in specific cells, the basidia, of the mushrooms that the two nuclei will fuse, just before meiosis (Figure 1). Consequently, sex in basidiomycetes is separated in time from gamete fusion, which is in contrast to almost all other sexual life cycles. Third, although the dikaryon cannot be fertilised by a second haploid nucleus, it retains the potential to donate nuclei to another monokaryon (Buller 1930, Quintanilha 1937). We systematically explore the potential for genomic conflict in this life cycle and discuss empirical evidence for the theoretical predictions.

\section{The monokaryon: gametes and sexual roles}

What are the male and female gametes in the basidiomycete life cycle (Anderson and Kohn 2007)? The answer to this question is important, since it is one of the determinants for genomic conflict. In the standard life cycle of basidiomycetes, two monokaryons reciprocally fertilise each other by simultaneously donating nuclei to, and receiving nuclei from, their mating partner (Figure 1). Ultimately, the donated nuclei are the male gametes as these fertilise the monokaryon without prior investment in growth. It is less obvious what the female gamete is. As each monokaryon can be fertilised in its entirety, the monokaryon can be considered as a single female 'super gamete'. Alternatively, as each cell of the monokaryon can be fertilised, the monokaryon can also be considered as a sexual organ and each of its cells as a female gamete. This is not just a theoretical argument, since a single monokaryon can be fertilised by multiple genetically different nuclei, from multiple monokaryons (Nieuwenhuis and Aanen 2012). However, because the monokaryon exhibits both a female and male role, i.e. the reception and donation of nuclei, respectively, and can grow vegetatively, the monokaryon also is a multicellular individual, albeit a special one, since each cell can become fertilised. So in essence, each cell of the monokaryon can behave as a female gamete and each nucleus as a male gamete.

\subsection{The female role - exposed to risks and bearing the costs}

Although the monokaryon needs to accept another nucleus to become fertilised and reproduce sexually, by doing so it exposes itself to risks and potential costs. At 'gamete fusion', the nucleus of the receiving monokaryon is providing a soma to the nucleus of its mating partner, while its partner provides only a nucleus. If the resulting dikaryon is 


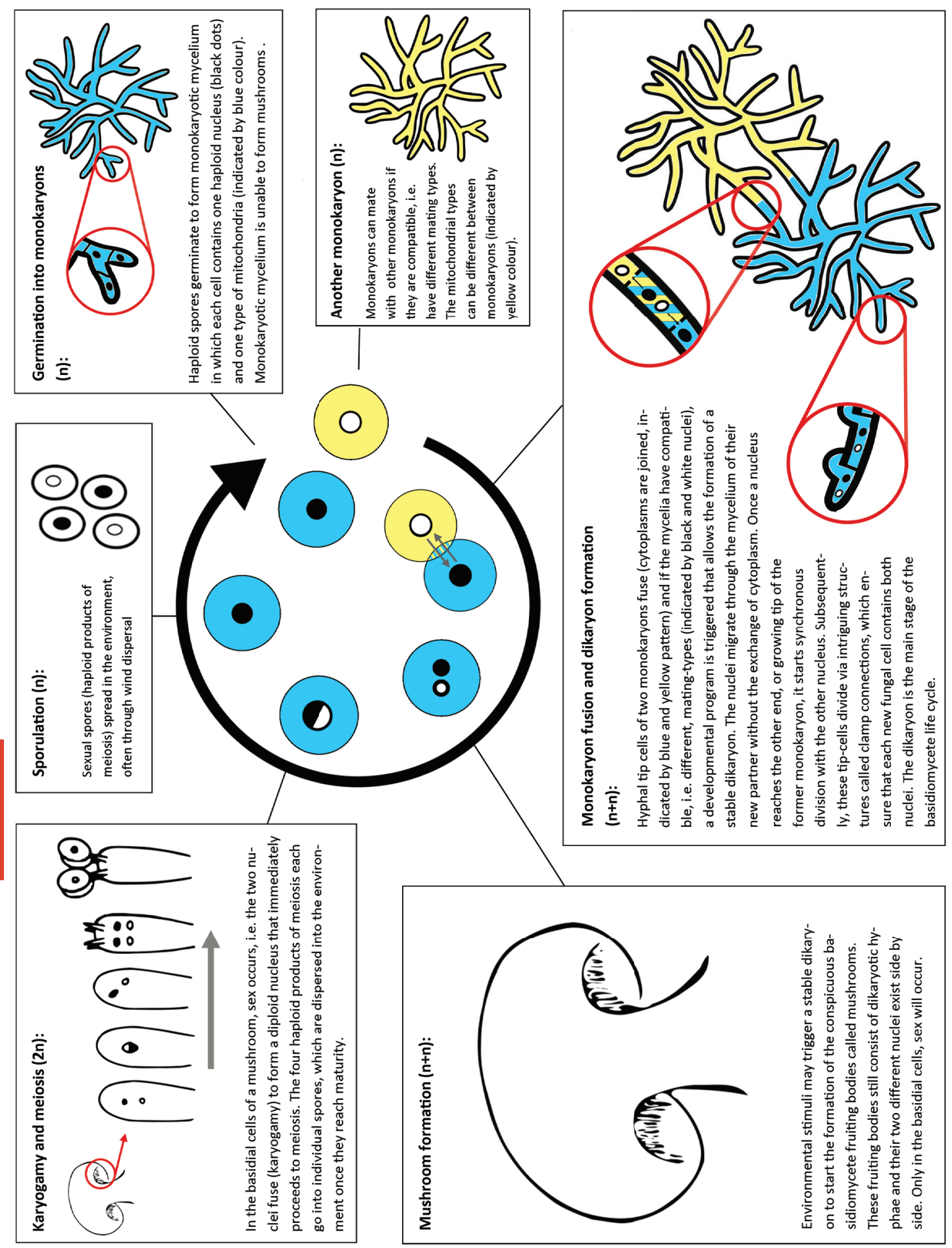




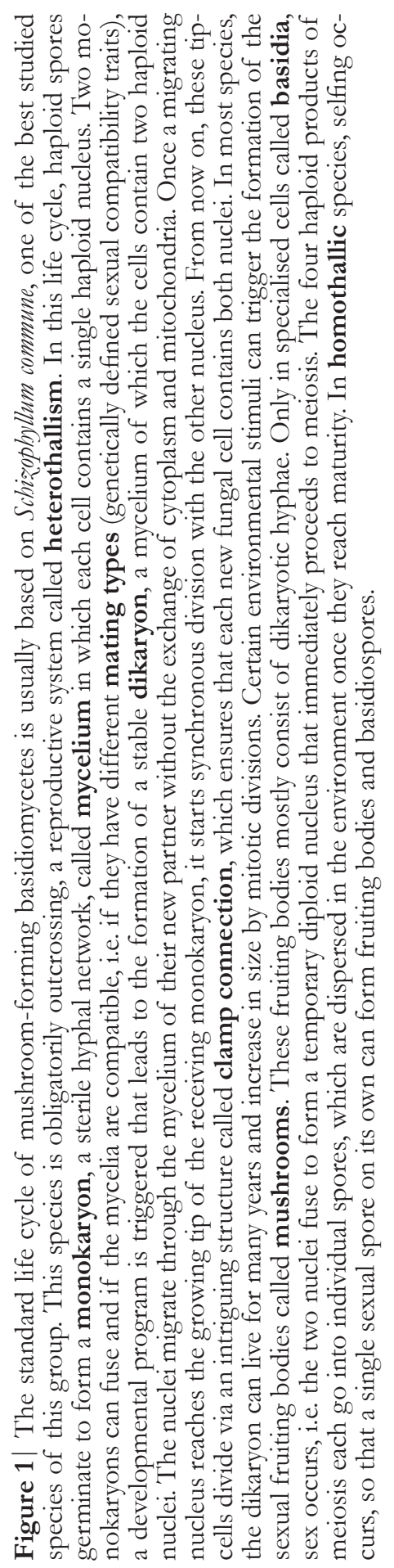


poorly functioning, the cost is borne by the receiving monokaryon, which contributed the cytoplasm, and which now has decreased or even lost its chance to reproduce. One example would be the invasion of selfish nuclei, which might take over the cytoplasm and eliminate the original nucleus. An observation in spore-trap experiments indicates that this might happen in Scbizophyllum commune (James and Vilgalys 2001). Also in the genus Armillaria, it has been shown that there can be hostile take-overs of one nucleus by a different one, although this is an exceptional genus as it has diploid nuclei (Rizzo and May 1994, Carvalho et al. 1995). Similarly, in pairings between selfing and outcrossing populations of the species Stereum birsutum replacement of the nucleus of the outcrossing population by a nucleus of the selfing population has been demonstrated (Ainsworth et al. 1990). Even without selfish elements, the new nucleus might carry genes that are not well adapted to the environment or not compatible with the original nucleus. This may render the dikaryon less adapted to the environment than the original monokaryon. Thus this "female role" of the monokaryon is believed to be one chance only, since the accepted nucleus cannot be aborted, even if the resulting dikaryon is maladapted.

Furthermore, although the dikaryon resulting from a mating between monokaryons cannot accept another nucleus, it can still donate one of its nuclei to a monokaryon (Buller 1930, Snider and Raper 1958), a phenomenon called the 'Buller phenomenon' or 'di-mon mating' (Quintanilha 1937). However, by accepting another nucleus, and forming a dikaryon, the receiving nucleus enters into a competition with the nucleus it just accepted for future success in di-mon matings.

So there are two potential costs of accepting a nucleus. The first arises from accepting a nucleus that results in a maladapted dikaryon. The second cost arises from accepting a more competitive nucleus that wins in the competition for di-mon fertilisations. Both costs should promote the evolution of female choice (Nieuwenhuis et al. 2011, Nieuwenhuis and Aanen 2012).

\section{Recognition of the sources of male gametes and its consequences}

The male role of a monokaryon is the donation of nuclei to its mating partner. However, there are also other sources of male gametes. First, sexual spores can act as male gamete if they land on an established monokaryon (James and Vilgalys 2001, Anderson and Kohn 2007, Nieuwenhuis et al. 2013). Second, as described above, the dikaryon resulting from a mating between monokaryons can still donate one of its nuclei to a monokaryon (Buller 1930, Snider and Raper 1958), the so-called 'Buller phenomenon' (Quintanilha 1937). The recognition of the various sources of male gametes has im- 
portant implications for the opportunities for sexual selection, genomic conflict and for the calculation of the cost of sex.

\subsection{Outcrossing basidiomycetes have a male-biased operational sex ratio}

Since the dikaryon cannot receive another nucleus, a di-mon mating is equivalent to a mating between a male and a female. Because of this, all populations of outcrossing basidiomycetes, which consist of 'male' dikaryons and 'hermaphroditic' monokaryons, have a male-biased operational sex ratio, which can increase the importance of sexual selection (Nieuwenhuis et al. 2011, Nieuwenhuis and Aanen 2012) (see also section 4.2: Nuclear competition during Buller phenomenon).

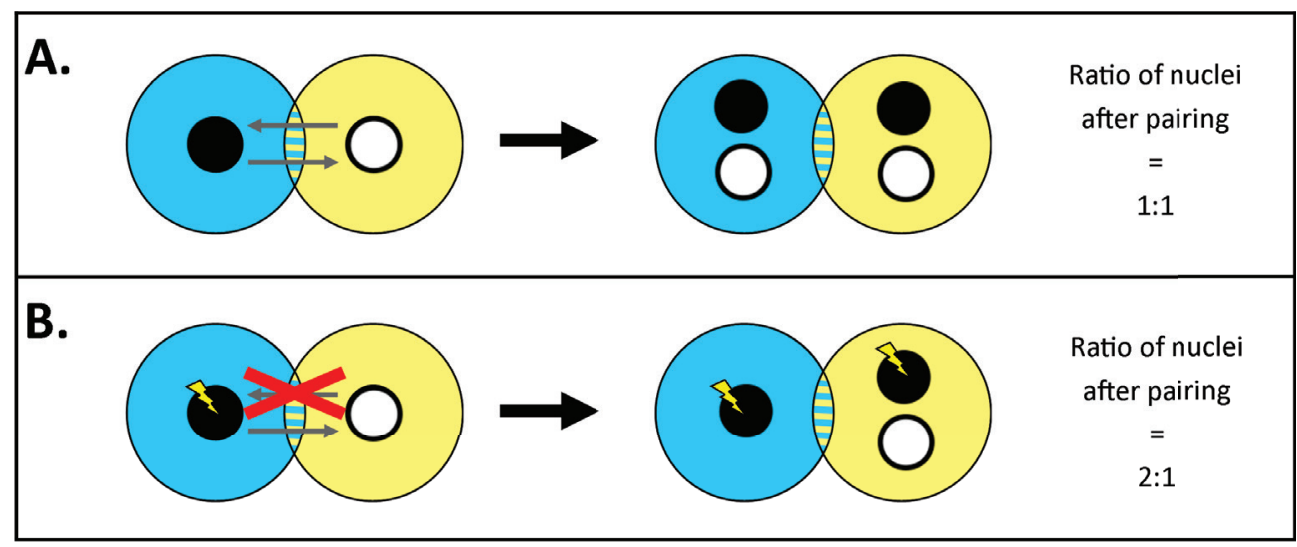

Figure $2 \mid$ A) Schematic illustration of a pairing between two monokaryons, where each monokaryon dilutes its genetic constitution by the acceptance of a nucleus, and compensates for the dilution by simultaneously donating nuclei to its partner monokaryon. B) As in A, with the difference that one of the monokaryons has gained a mutation that prevents dilution of its genetic constitution, by inducing asexual reproduction or sexual reproduction via selfing, while still being able to donate a nucleus. While the ratio of black and white genome after pairing has changed from 1:1 to $2: 1$, the ratio of black and white genome in the spores has changed from 1:1 to $3: 1$, assuming that both parts of the mycelium produce equal numbers of spores.

\subsection{The one-and-a-half-fold cost of sex}

The hermaphroditic nature of pairings between two monokaryons can provide the conditions for the selection of ultra-selfish genes. As explained above, in the regular lifecycle of mushroom-forming basidiomycetes, the monokaryon simultaneously donates and accepts another nucleus. By accepting another nucleus, a monokaryon dilutes its own genetic constitution, but compensates for this genome dilution by donating nuclei to its partner monokaryon. Imagine a mutation in a monokaryon that prevented its genomic dilution by prohibiting the acceptance of another nucleus, and enabled asexual reproduction or selfing, while still allowing the fertilisation of another monokaryon (Figure 2). Everything else being equal, the relative benefit of this mutation would be $50 \%$ relative to its wildtype allele (Aanen and Hoekstra 2007). Since this benefit is 
shared with all other genes of that nucleus, there is no genomic conflict at the level of the monokaryon (see Aanen and Hoekstra (2007) for a more extensive discussion).

\subsection{Changing life cycles}

A similar argument can be made for the initial benefit of another, similar kind of mutation occurring in a dikaryon of an obligatorily outcrossing (so-called heterothallic - a mating system where mating can only occur between gametes with different mating types) species. If this mutation changes the outcrossing life cycle into a selfing (homothallic or secondarily homothallic - a mating system where mating can occur between gametes with identical mating types) or asexual life cycle, all spores produced by this dikaryon contain the mutation. If such a mutant still allows the dikaryon to donate one of its nuclei to a monokaryon, it theoretically gains a benefit over the wildtype. The exact magnitude of this benefit is hard to calculate since it depends on the frequency of di-mon matings in a population, which we do not know. The example on Stereum birsutum discussed above, in which a nucleus of a selfing population is shown to have been donated to monokaryons from an outcrossing population, illustrates that such mutations do exist (Ainsworth et al. 1990).

Agaricus bisporus is one of the mushroom-forming basidiomycetes in which an outcrossing mating system evolved into a (partially) selfing (secondarily homothallic) mating system (Raper et al. 1972). In this species, there are two interfertile varieties that both produce dikaryotic and monokaryotic spores, but in a different ratio. In $A$. bisporus var. bisporus (all present-day commercial varieties and most wild varieties) two meiotic products, i.e. two nuclei, are packaged into one spore in over $90 \%$ of the basidia in such a way that both parental mating types are represented in each spore (Evans 1959, Saksena et al. 1976, Callac et al. 1993, Kerrigan et al. 1993). As both parental mating types are present in these dikaryotic spores, they are self-fertile. In contrast, A. bisporus var. burnettii (a subspecies isolated from the Californian desert) predominantly produces four monokaryotic spores, which are therefore not self-fertile (Callac et al. 1993, Kerrigan et al. 1993).

As explained above, the transition from obligate outcrossing (heterothallism) could be explained by an initial fitness gain of the selfing (homothallic) variety in an outcrossing (heterothallic) population (Aanen and Hoekstra 2007). However, this predicted fitness gain might be severely reduced as the new life cycle will lead to inbreeding and increased homozygosity. Yet, this particular species appears to have evolved a way to minimise the effects of inbreeding by redirecting meiotic crossovers almost exclusively to its chromosome ends (Kerrigan et al. 1993, Sonnenberg et al. 2016). By having crossovers at chromosome ends, the offspring of these mushrooms have practically intact parental chromosomes. Combined with predominantly non-sister nuclei pairing in spores, this ensures that $\sim 90 \%$ of the var. bisporus spores practically only differ from their paren- 
tal genotypes by a reshuffling of homologous chromosomes over the two nuclei. All chromosomes combined still form the same genome. Hence, genetically, the resulting mushrooms are almost identical to the parental mushroom, which limits the detrimental effects of inbreeding.

\section{The dikaryon: genomic conflict due to mitochondrial competition}

During a mating between two monokaryons, a combination of migration and nuclear division transforms the two monokaryons into a single dikaryon in terms of nuclear genomes. All cells of the dikaryon contain two nuclei, one of each monokaryon. In contrast, the mitochondria of each monokaryon do not migrate, so that the two mitochondrial types of the resulting dikaryon are separated in space (Figure 3). This gives rise to possible competition, among the mitochondrial haplotypes from the two original monokaryons, within the newly formed dikaryon.

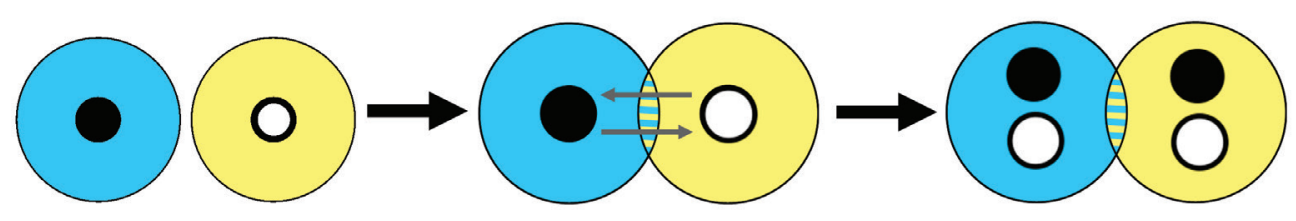

Figure $3 \mid$ Schematic representation of a mating between two monokaryons. In a mating between two monokaryons, both nuclei (one white, one black) migrate to fertilise their respective partner mycelium. The cytoplasms, and thus the mitochondria (blue and yellow) are not exchanged, except for in the narrow interaction zone in which the hyphal tips fuse (blue and yellow stripes). Consequently, a single dikaryon is formed in which all cells have the same nuclear genotype, but may have different mitochondrial genotypes.

Since most cells of the dikaryon contain only a single type of mitochondria, and each cell potentially can give rise to a mushroom, cytoplasmic inheritance is doubly uniparental: both monokaryons involved in a mating can potentially transmit their cytoplasm to the sexual spores, but normally only a single type per spore (Aanen et al. 2004). In this life cycle, within-cell competition between genetically different mitochondria is limited, since the only cells that contain the two types of mitochondria are the fused cells at the initiation of mating (Figure 1). However, at the dikaryon level, the two types of mitochondria do compete over transmission. This is a peculiar situation: although there is restricted cytoplasmic exchange, there is nevertheless enduring physical contact between cells with two types of mitochondria. If individual mitochondria can increase their relative chance to be included in the spores and if this occurs at a cost of dikaryon fitness, this leads to genomic conflict for two reasons:

I. A mitochondrial gene can be selected at the level of the cytoplasmic genome but selected against at the level of the dikaryon; 
II. Because nuclei are homogeneously distributed in the dikaryon, nuclear fitness is directly dependent on dikaryon fitness. A reduction in dikaryon fitness because of intra-dikaryon mitochondrial competition is therefore directly in conflict with nuclear interests.

One theoretical possibility for ultra-selfish behaviour of mitochondrial genes is via the induction of male sterility. A monokaryon normally both accepts its partner's nucleus and donates its own, which are female and male roles, respectively. Theoretically, a mitochondrion that can prevent the male role of the monokaryon it resides in while maintaining its female role (Cytoplasmic Male Sterility, or CMS) will have a selective advantage over a partner mitochondrion that does not do so for two reasons (which are not mutually exclusive):

I. Such a mitochondrion will monopolise the spores, because fruiting in the other section of the mycelium will be prevented;

II. In most basidiomycetes, the relative growth rate of a dikaryon is higher than that of a monokaryon (Swietzynski and Day 1960, Kues 2000). Therefore, even postponing male function relative to female function can be advantageous for an individual mitochondrion.

Aanen et al. (2004) have modelled the evolution of mtDNA-induced male sterility. In their model, there were male-sterile and male-fertile mtDNAs and nuclear determinants specifying either resistance or susceptibility to cytoplasmic male sterility. In a monokaryon with a resistant nucleus, the effect of mtDNA-based male sterility is nullified, and nuclear migration occurs irrespective of whether the other monokaryon is male sterile or male fertile. The model explained data obtained for the genus Hebeloma reasonably well (Aanen et al. 2004).

In plants, cytoplasmic male sterility is well established and leads to gyno-dioecy, a mating system with female and hermaphroditic plants (cf. Frank 1989, Budar et al. 2003). In all described cases, CMS is encoded by mitochondrial mutations, while resistance genes exist in the nuclear genome. For basidiomycetes, the question is what possibilities mitochondria would have to induce male sterility. A theoretical mechanism for male sterility is that mtDNA mutations somehow block the mating pheromone receptors of the other monokaryon, or block the production of the pheromones of the male-sterile strain. Also, if a mitochondrion can induce the dikaryon to produce more mushrooms in the part of the mycelia with that mitochondrion, it will increase its proportion of spores, even without causing male sterility. However, it remains to be demonstrated that cytoplasmic male sterility plays a general role in basidiomycete fungi. 


\section{The dikaryon - nuclei living apart together: genomic conflict due to nuclear competition.}

\subsection{Nuclear competition during vegetative growth and asexual reproduction}

Another source of genomic conflict arises from competition among the nuclei within the dikaryon during vegetative growth and asexual propagation (Buss 1987, Ramsdale 1999). If the replication of the nuclei in the mycelium is not regulated, one nucleus can divide faster than the other, increasing its relative abundance within the mycelium, even if this decreases the fitness of the mycelium. A study on vegetative growth rates of monokaryons and dikaryons of Heterobasidion parviporum confirmed that it is possible for a nucleus to dominate the dikaryon, even when it leads to a decreased growth rate (James et al. 2008).

Alternatively, a nucleus can be opportunistic in positioning itself towards the hyphal tip. Because growth in filamentous fungi occurs at the edge of the mycelium, those nuclei that position themselves at the hyphal tips take part in growth and can replicate (Xiang and Fischer 2004). In most ascomycetes and some basidiomycetes, mitotic growth is not well regulated (Gladfelter and Berman 2009). These fungi can form multinucleate heterokaryotic cells, in which the ratios between the two types of nuclei can deviate strongly from 50-50 (Davis 1959, Hui et al. 1999, James et al. 2008). This can lead to the escape of monokaryotic hyphae (e.g. Agaricus bisporus; (Wang and Wu 1974)) and to the production of monokaryotic asexual spores (oidia) favouring the nuclear type that is in the majority (e.g. Heterobasidion annosum, Heterobasidion parviporum, Pholiota microspora (Pholiota nameko) (Arita 1979, Ramsdale and Rayner 1994, Ramsdale and Rayner 1996, Hui et al. 1999, James et al. 2008)). In contrast, in Termitomyces, another basidiomycete fungus with multiple nuclei per cell, no monokaryotic escapes have been found (Nobre et al. 2014). Similarly, in some of the mutualistic fungi cultivated by fungus-growing ants, multiple nuclei are present per cell. However, in contrast to Termitomyces, more than two haploid genomes are maintained within a single mycelium, although at present it is unknown if those genomes are distributed between different haploid nuclei, or within polyploid nuclei (Kooij et al. 2015).

In most basidiomycete species, the formation of so-called clamp connections between dikaryotic cells prohibits deviations from a one to one ratio among the two nuclear types during somatic growth of the dikaryon (Figure 1). Nevertheless, if monokaryotic spores are formed during asexual spore production, one nuclear type could still be overrepresented in species with clamp connections. It is striking though that the two nuclei of a dikaryon cell appear to change position after each conjugate division (Iwasa et al. 1998). Although asexual reproduction probably is less important in most species of basidiomycetes than in ascomycetes, it is tempting to consider this highly regulated 
change in position of the nuclei as a specific 'policing' mechanism at the level of the dikaryon to reduce the risk that one nuclear type can monopolise asexual monokaryotic spores. Nevertheless, the two possible kinds of asexually formed monokaryotic spores by a dikaryon with clamp connections can show strong deviations from 50-50 ratio (Hui et al. 1999, Kitamoto et al. 2000). If the gene that biases the nuclear ratio comes at a cost for the total number of spores produced by the dikaryon, there is genomic conflict.

\subsection{Nuclear competition during Buller interactions}

In section 1a we predicted that a monokaryon will be choosy in accepting a nucleus, since the receiving nucleus will enter a competition with the accepted nucleus for future success in Buller pairings. In this section, we address the potential implications for genomic conflict of this within-dikaryon inter-nuclear competition for di-mon matings (Buller 1930). Since most populations of basidiomycetes contain hundreds of mating types, the vast majority of nuclei are compatible. So, both nuclei of a dikaryon usually will be compatible with an unrelated monokaryon, and will compete to fertilise it in a di-mon interaction. If there would be no difference between the two nuclei, each would have $50 \%$ chance to fertilise the monokaryon. However, it has been found that the success of nuclei often strongly and consistently deviates from a one to one ratio in favour of one of the two, even if both are compatible with the monokaryon (Ellingboe and Raper 1962, Crowe 1963, Ellingboe 1964, Nieuwenhuis et al. 2011). In theory, such success in Buller matings could be due to a mutation (or multiple mutations) that increases the success of its containing nucleus even if that same mutation reduces dikaryon fitness as a pleiotropic effect. This is a conflict between a gene selected at the level of the nucleus in competition with another nucleus, and all other nuclear genes, which depend on dikaryon fitness (except for half of the genes residing in the same nucleus as the mutation, but only until recombination cuts that linkage). But how large can this conflict be?

Let a mutation $a$ give a nucleus a benefit $x$ in competition with a nucleus with the wildtype allele, so that its success in Buller matings is $0.5+x(0 \leq x \leq 0.5)$ relative to the wildtype, but it decreases the relative fitness $(w)$ of the dikaryon it finds itself in with y, so:

$\begin{array}{ll}\mathrm{w}_{\mathrm{wt}}=1+0.5 * 1=1.5 & \text { (See Figure 4A) } \\ \mathrm{w}_{\mathrm{a}}=1-\mathrm{y}+(0.5+\mathrm{x})(1-\mathrm{y}) & \text { (See Figure 4B). }\end{array}$

If

$1-\mathrm{y}+(0.5+\mathrm{x})(1-\mathrm{y})>1.5$,

$a$ will be selected. 
Now we consider the maximum possible value of $x(0.5)$, to calculate the maximum fitness reduction $y$ for the mutation still to be selected:

$1-\mathrm{y}+1-\mathrm{y}=1.5 \rightarrow \mathrm{y}=0.25$.

So, the maximum tolerable fitness reduction for a mutation that increases the success in Buller pairings to $100 \%$ is 0.25 .

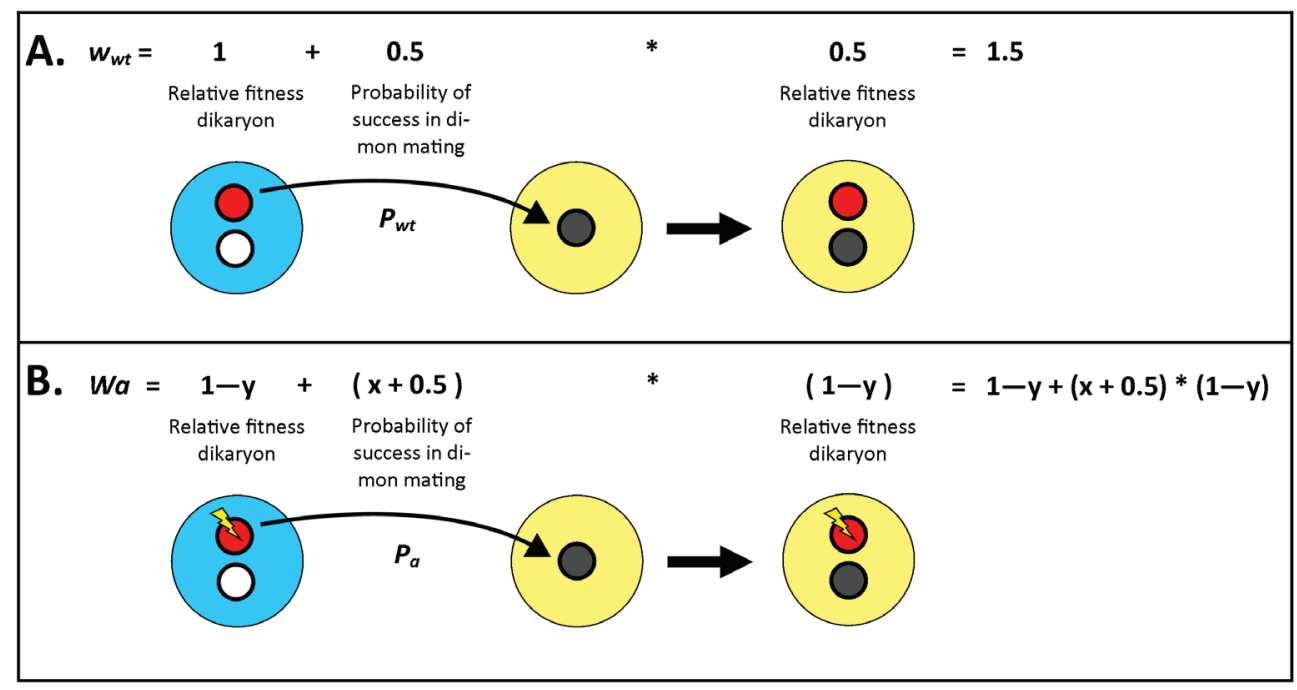

Figure 4 | Schematic illustration of a di-mon pairing, where the two nuclei of a dikaryon compete to fertilise a monokaryon.

The calculation above ignores at least two important complications. First, the selection of an ultra-selfish gene is frequency dependent. So, to calculate its fitness, we would need to consider the change in frequency during selection to find its equilibrium frequency. Second, assuming that mildly ultra-selfish genes may go to fixation, they will form the starting situation for a possible new round of selection for other ultra-selfish mutations. So, the maximum value calculated above only applies to one particular time point, and for one possible kind of interaction. However, the major force determining the selective advantage of a mutation leading to an increased male performance in dimon matings, is the -unknown- frequency of di-mon mating events in nature. Using experimental evolution in Schizophyllum commune for increased male fertility, increased male mating success could be selected, but generally no clear trade-offs were found with other fitness components, such as growth rate (Nieuwenhuis and Aanen 2018). 


\subsection{Possible mechanisms for winning the competition between nuclei}

\subsubsection{Mating types}

Basidiomycetes generally have many mating types, up to thousands (Raper 1966). In most basidiomycetes the mating type is determined by two loci: a locus encoding a homeodomain transcription factor (HD), (the "A" locus), and locus, encoding pheromones and pheromone receptors (P/R), (the "B" locus) (see Freihorst et al. (2016) for an excellent overview). Two nuclei are compatible if the alleles at both mating type loci are different; therefore, the probability of being compatible is mainly determined by the number of mating types at the locus with the lowest number of variants. The mating type genes have been found to evolve faster than genes involved in other conserved functions (May et al. 1999, James et al. 2004) and especially at the P/R locus there is a large variety of pheromone alleles. It has most often been inferred that the enormous diversity in mating types allows for maximum outbreeding, whilst it reducing sibling compatibility. Almost any randomly encountered individual will have a different mating type whereas only $25 \%$ of the progeny of the same fruiting body will be compatible (Badalyan et al. 2004). However, this hypothesis does not account for the high degree of redundancy, i.e. usually multiple compatible pheromone-receptor interactions are found between alleles, whereas a single compatible interaction is sufficient. Therefore, Nieuwenhuis and Aanen (2012) proposed the alternative hypothesis that the mating-type genes are a target of sexual selection in Basidiomycete fungi (see also James 2015). More specifically, they hypothesised that the redundancy in pheromones at the $\mathrm{P} / \mathrm{R}$ locus is a consequence of sexual selection (Nieuwenhuis and Aanen 2012). As explained above, in di-mon matings competition can arise between the two nuclei of a dikaryon that might be costly to the dikaryon as a whole. It would be interesting to test in future studies i) if the $\mathrm{P} / \mathrm{R}$ mating type locus is indeed responsible for the observed deviation in success between the two nuclei of a dikaryon in a Buller mating and ii) if the locus itself, or any genes linked, have a negative effect on overall dikaryon fitness.

\subsubsection{Nucleus-specific and parent-of-origin effects on gene expression}

Since there is no genetic sex determination in basidiomycetes, potential conflicts could be combatted by differential gene regulation in a way analogous to genomic imprinting (the differential expression of alleles of a gene depending on its parent of origin) found in mammals and plants (Reik and Walter 2001, Kohler and Weinhofer-Molisch 2010). However, with the marked difference that, whereas in other organisms the imprint is determined by the sex of the meiotic parent, in basidiomycetes the difference in gene regulation would have to depend on the sex role taken by the monokaryon at fertilisation.

In di-mon matings, the two male gamete types, i.e. the nuclei of the dikaryon, compete for the fertilisation of the monokaryon. This male-male competition is analogous to 
sperm competition, with the fundamental difference that it occurs within the cell in which the two competitors are together and generally assumed to cooperate. Potentially, one nucleus could regulate gene expression in the other nucleus, in such a way that it reduced the male potential of its competitor nucleus. Especially the receiving monokaryon might have most power to suppress the future male role of the fertilising nucleus, since the receiving nucleus contributes the cytoplasm to the initial dikaryon. Given that the haploid monokaryotic mycelium does not need to form a dikaryon for survival and vegetative growth, the hypothetical extreme-case scenario is that the second nucleus is active only during sexual reproduction. This putative mechanism would remove all competition in Buller matings, while leaving the outcrossing advantage of sexual reproduction intact.

A study of the ascomycete fungus Neurospora tetrasperma has shown that gene expression levels can differ between the two nuclei in a dikaryon (Samils et al. 2014). Unlike most ascomycetes, the vegetative mycelium of $N$. tetrasperma commonly consists of two nuclear types, but the ratio of the two nuclei was found to deviate from one to one. However, the relative gene expression of a few investigated genes did not reflect the ratio of each nuclear component in the mycelium (Samils et al. 2014). Although this example is an ascomycete species, it shows that the relative gene expression of a nucleus does not necessarily correlate with its frequency in the mycelium.

The differences in gene expression between the two nuclei are not necessarily the result of competitive interaction. If one nucleus is better adapted to the environment, the dikaryon as a whole could benefit if that nucleus became dominant, in terms of gene expression. Under such scenario differential gene expression would be adaptive for both nuclei. Alternatively, differences in gene expression between nuclei are the result of competition. Recently, research has shown that selfish behaviour of nuclear variants within the mycelium of the ascomycete Neurospora crassa can occur. In an evolution experiment, cheater nuclei were selected that had a relative benefit in competition with wild-type nuclei, at the cost of the total number of spores produced by the mycelium (Bastiaans et al. 2016). However, the mechanism by which these differences in competitive success are achieved remains to be explored.

\section{General discussion}

The specific aspects of the sexual life cycle of mushroom-forming basidiomycetes leave room for the selection of ultra-selfish genetic elements that are in conflict with the rest of the genome. This implies that basidiomycete 'organismality', i.e. the extent to which the parts composing a social group, in this case a multicellular individual, work together for the common whole, is lower than in other multicellular organisms (Queller and 
Strassmann 2009). A corollary of this is that organismal fitness may be suboptimal, and that differentiation is less irreversible than in animals and plants.

In contrast to a diploid organism with a single fused, diploid nucleus and one type of mitochondrial genome (and other cytoplasmic organelles) per cell, the dikaryon consists of multiple genetic entities that form an 'unholy marriage' as they can still pursue their own selfish interest to some degree, even if this comes at a cost of the dikaryon as a whole. The two genetically different haploid nuclei of the dikaryon remain separate until just before meiosis and the dikaryon might also be a mosaic for mitochondrial types, although most cells will contain only a single type. So, sex in basidiomycetes is separated in time from gamete fusion, and the nuclei remain separate for most of the vegetative stage until just before meiosis, facilitating 'eternal triangles' during the dikaryon stage. Furthermore, gametes in the basidiomycete life cycle are not single cells, but multicellular organisms that mate in a hermaphroditic fashion.

There is an interesting parallel between basidiomycetes and mosses. In mosses, a haploid gametophyte produces eggs that get fertilised by sperm produced by the same or a different gametophyte to produce a diploid sporophyte. Also, after fertilisation, the gametophyte 'mother' supports that diploid sporophyte, which grows on top of her (Haig and Wilczek 2006, Haig 2016). Similar to the monokaryon in basidiomycetes, in the moss life cycle, the haploid 'mother' thus invests before fertilisation and supports an unrelated haploid genome of her mating partner after fertilisation. In both life cycles, conflicts between male and female haploid 'interests' may be played out both before and after gamete fusion.

The first main category of possible genomic conflicts we identified is between mitochondrial and nuclear DNA, due to the different inheritance modes of these genomes. A possible result of this type of conflict is the evolution of mitochondrion induced male cytoplasmic sterility. However, there is not much evidence that this type of conflict is important, and it seems hard to imagine that the mitochondrial DNA has sufficient possibilities to induce male sterility in the basidiomycete life cycle.

The second main source of genomic conflicts is nuclear competition. We have pointed out that a monokaryon can simultaneously behave as a male (each nucleus) and female gamete (each monokaryotic cell). Once fertilised, the resultant dikaryon has lost its female potential, but retains its male potential via so-called di-mon (or Buller) pairings. The consequential male-biased operational sex ratio, combined with the potential cost of accepting a nucleus, could lead to selection for ultra-selfish mutations, providing a benefit to a nucleus in competition with the other nucleus. This process can also be interpreted in terms of sexual selection. The one theoretical prediction is that the nuclei in the dikaryon are in competition to fertilise monokaryons, which may favour traits 
that provide a selective benefit at the level of the nucleus, but that are harmful for the dikaryon. We have calculated the maximum tolerable costs for such mutations using some simplifying assumptions, and have shown that these costs can be significant. The other prediction is that the monokaryon will be under selection to be critical to the nucleus she accepts, as this determines her fitness. A possible mechanism for winning the competition among nuclei is via the pheromones, encoded by one of the mating-type loci, and we have argued that the extreme redundancy of pheromones compared to pheromone receptors that is observed may be a consequence of this selection process.

Another consequence of the 'living apart together' of the two haploid nuclei in the dikaryon is that competition occurs to enter the asexual spores. Even though asexual reproduction is less important in basidiomycetes than in many other fungi, there are some examples where one nucleus has a higher representation in the asexual spores.

A different type of ultra-selfish mutations is a mutation that monopolises the spores. A mutation that turns a monokaryon or a dikaryon into asexual or selfing (homothallic) reproduction, whilst retaining the possibility to donate nuclei, would be selected. This selective benefit could be the explanation for frequent changes in life cycles seen in some basidiomycete groups, such as the genera Agaricus, Coprinus and Mycena.

The analysis in this paper is largely theoretical. Most research on competition and conflict in the mushroom life cycle has been in laboratory settings with only a small set of model organisms. We see two main lines of progress for future research. First, experimental evolution may provide the experimental tests of theoretical predictions, and some progress has recently been made (cf. Clark and Anderson 2004, Nieuwenhuis and Aanen 2018). Second, due to progress in genetic and genomic techniques, the possibilities of studying natural populations and non-model organisms will increase (cf. Anderson and Catona 2014). These recent advances will allow us to assess the relevance of the potential sources of genomic conflict in the basidiomycete life cycle that we identified in this paper. 


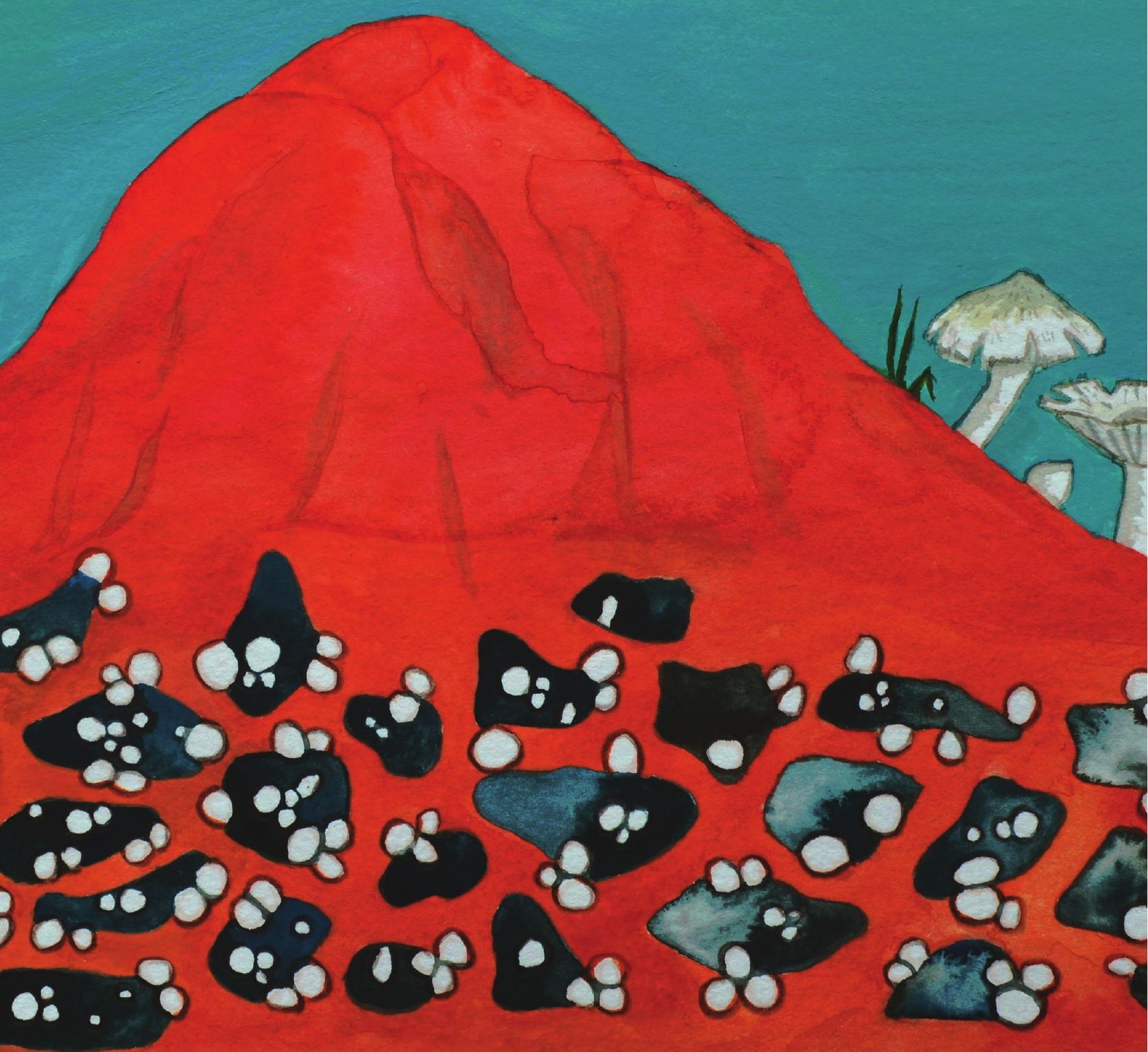




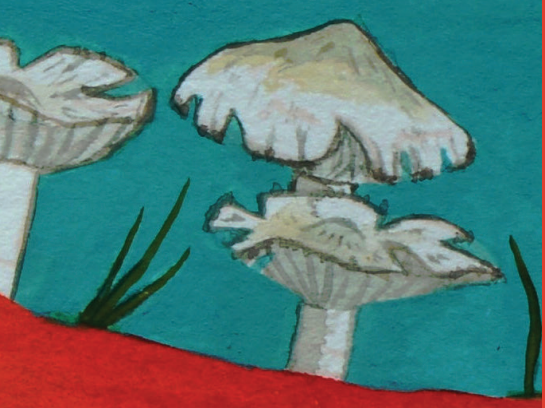

General discussion

Sabine M.E. Vreeburg

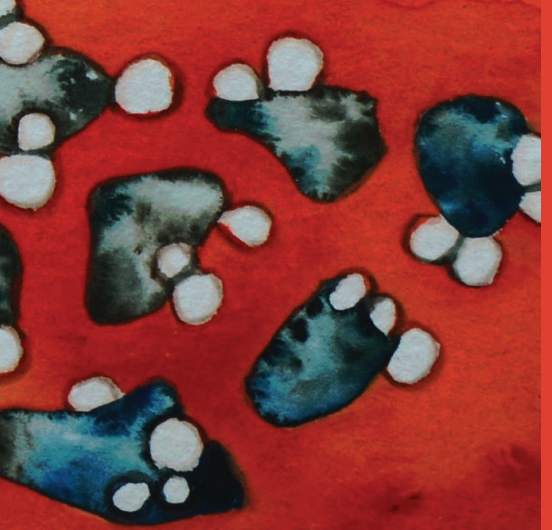


At the heart of my argument is the simple observation that the history of life is a history of the elaboration of new self-replicating entities by the self-replicating entities within them (or the incorporation of some selfreplicating entities by others).

- Buss (1987) 


\section{Introduction}

Often, when people talk about evolution they mean adaptive evolution through natural selection. For adaptive evolution to occur on a certain trait three requirements need to be fulfilled: 1.) the trait must vary in the population, 2.) the variation in the trait must affect how well an individual carrying the trait survives and reproduces under a selection pressure, 3.) the variation must be heritable. A great example of evolution through natural selection was found by Rosemary and Peter Grant who studied the two main species of Darwin's finches (Figure 1) on the Galapagos island Daphne Major. Amongst other traits, they measured beak size of the birds (beak size is a trait that varies in the population of birds). Then, one year the rainy season brought very little rain and it diminished the bird populations, especially that of the medium ground finch, one of the species they studied. These birds were usually eating smaller, softer seeds, that were no longer available due to the drought. Only ground finches with larger beaks were capable of breaking larger seeds and they survived and got to reproduce. In the next generation the average beak size was larger, because beak size is heritable and only birds with larger beaks got to reproduce (Grant 1991, Grant and Grant 2003).

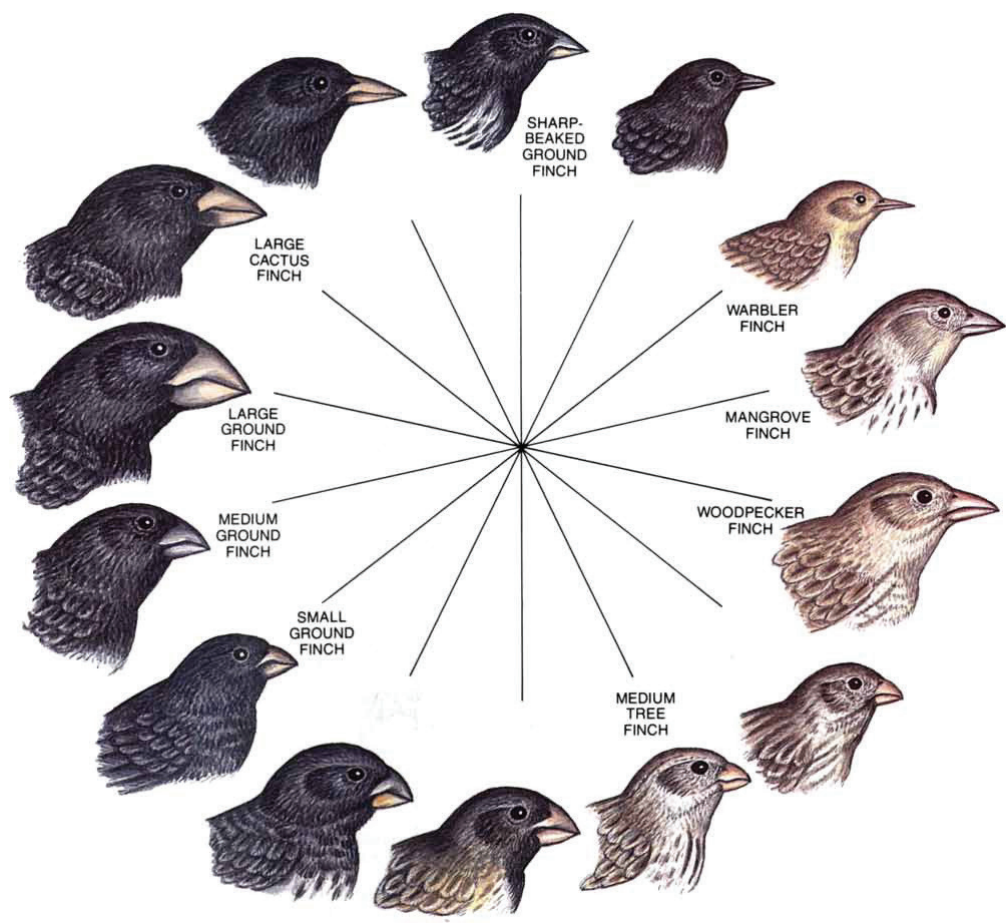

Figure 1| Darwin's finches. Rosemary and Peter Grant studied the two major populations on the Galapagos island Daphne Major: the medium ground finch (Geospiza forti) and the cactus finch (G. scandens). Figure from Grant (1991). 
Above I give the relatively intuitive example of how natural selection can act on individuals in a population of birds. However, these birds are made of individual cells on which natural selection can act directly too. Within the cells there are mitochondria on which natural selection can act too. Within the nuclei of the cells there is DNA containing genes on which again natural selection can act. This is the hierarchical fashion in which life is organised, as Buss so wonderfully phrased in the quote above. At higher forms of organisation each self-replicating entity consists of smaller entities that are or were once self-replicating.

As long as the interests of the smaller, or lower-level, entities align with the interest of the larger entity, or higher-level organisation, the cooperation between lower-level entities can continue (Buss 1987, Maynard Smith and Szathmáry 1995). This is the case if the fitness of the lower level is correlated with that of the higher level; this can for example occur if the smaller entity loses the ability to replicate independently and can only replicate as part of the whole. However, lower-level selection can still oppose higher-level organisation. Thus to stably maintain higher-level organisation, mechanisms are necessary to shift selection from the lower-level entity to the higher-level organisation (Buss 1987). Regulated cell division in multicellular organisms is such a mechanism.

An example of lower-level selection opposing higher-level organisation is the development of cancers. A cell of a multicellular organism can gain a mutation that allows the cell to escape from the normally regulated division. This mutation allows the cell to replicate faster and thus increase in number at the cost of the other cells. At the lower-level (level of the cell) there is selection for the mutation that increases the direct advantage of the individual cell, even though this is at the cost of the survival of the multicellular organism that holds the cell. Buss proposed a mechanism through which higher-level organisation in multicellular organisms is preserved, despite the lower-level selection for cancerous replication. Through early segregation of germline and soma, cancers in somatic cells are evolutionarily dead ends, since they are not passed on to the next generation of the multicellular organism (Buss 1987).

Interestingly, the principles that govern the (in)stability of higher-level organisations, e.g. alignment of reproductive interests of the lower level entities, are similar for all forms of higher-level organisation (Buss 1987, Maynard Smith and Szathmáry 1995). For all these transitions of lower-level entities to higher-level organisations, including symbiotic mutualisms such as the one that I studied in this thesis, the same question can be asked: "Why not did natural selection, acting on entities at the lower level ..., disrupt integration at the higher level?” (Maynard Smith and Szathmáry 1995).

In this thesis I studied stabilising mechanisms and possible conflicts at different levels of organisation in the ancient symbiosis between termites of the subfamily Macro- 


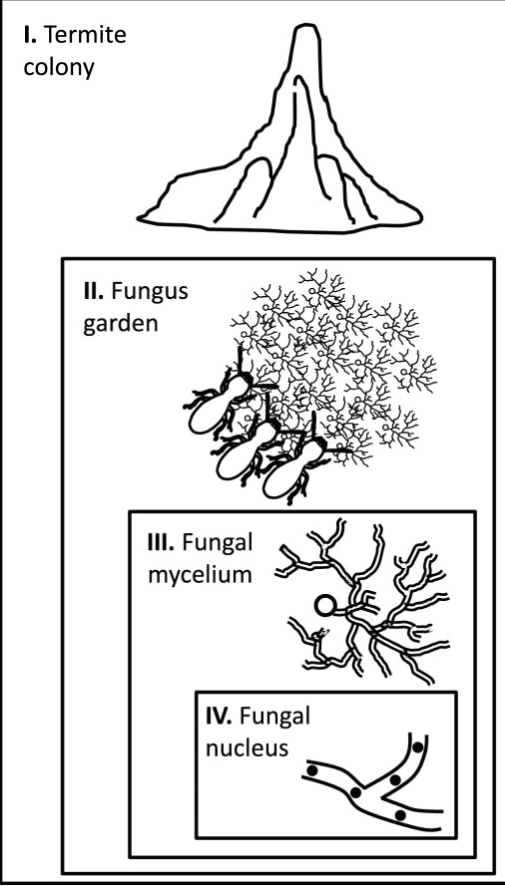

Figure 2| The levels at which selection can act on Termitomyces fungi in the termite-fungus symbiosis. Natural selection can act at the level of: I.) the termite colony, II.) individual fungus combs within the colony, III.) fungal individuals within a termite colony (when multiple individuals are present e.g. presumable at the founding of a colony), IV.) fungal nuclei within a mycelium. Figure from Nobre et al. (2014). termitinae and their basidiomycete fungi of the genus Termitomyces (Figure 2). At the organisational level of the fungus-growing termite colony, or the mutualism, we studied how, despite the seeming lack of alignment of reproductive interests between the two partners, the mutualism could remain stable through possible control of symbiont dispersal (chapter 2; Vreeburg et al. 2020) and co-evolution between host and symbiont (chapter 3; da Costa et al. 2019). Also we explored whether the symbiotic life-style of Termitomyces has had an effect on its recombination rate, as hypothesised by Law and Lewis (chapter 4; Law and Lewis 1983). In the same chapter we provided a tool for further studies of co-evolution (chapter 4). Finally, at the organisational level of the basidiomycete mycelium, we explored the possibilities for conflict between the mycelium as a whole and the individual nuclei and its mitochondria (chapter 5; Vreeburg et al. 2016). Here I will discuss the findings of this thesis in the light of the stability of higher-level biological organisation involving or within Termitomyces fungi.

\section{How is the conflict over reproduction mitigated?}

In most fungus-growing termite species, including the species that we studied - Macrotermes natalensis - there is independent reproduction between host and symbiont (chapter 2; Sands 1960, Johnson et al. 1981, Sieber 1983, Korb and Aanen 2003, De Fine Licht et al. 2006, Vreeburg et al. 2020). Consequently, there is no complete alignment of reproductive interest between the lower-level entities (termite and fungus) and the higher-level organisation (the mutualism). Frank (1996) showed that in general, in host-symbiont interactions there is a conflict of interest between host and symbiont over the dispersal of the symbiont and that under certain conditions host control over symbiont dispersal could evolve (Frank 1996). As fungal dispersal from the termite colony in those species with horizontal symbiont transmission likely only happens through fungal fruiting (see chapter 1), we assume 
that control over symbiont dispersal is equal to control over symbiont sexual reproduction. Whether the termite host controls dispersal of their fungal symbionts despite "allowing" horizontal symbiont transmission is currently unknown, even though there are indications that this is indeed the case. I will discuss these indications below.

The fruiting bodies, mushrooms, of Termitomyces fungi are usually found on active termite colonies and thus are likely part of the regular Termitomyces life cycle (see chapter 1, Figure 6). I would like to stress this point, because a common misconception is that Termitomyces mushrooms are only rarely found, and sometimes that they are only found on dying colonies. The origin of this misconception probably lies in the comparison with the convergently evolved ant-fungus symbiosis in which all ant species mainly transmit their fungus vertically and mushrooms are rarely observed (Mueller 2002).

One indication that termites control mushroom formation in their Termitomyces fungi is that, as multiple researchers noted, the appearance of the fruiting bodies coincides with the appearance of the first workers from newly founded termite colonies (Johnson et al. 1981, Kone et al. 2011). At this time the newly founded colonies need to re-establish the symbiotic relationship with a fungal symbiont (Johnson et al. 1981, Darlington 1994). Additionally, Kone et al. (2011) observed that only those termite colonies that produced alates - i.e. colonies that reproduced - produced mushrooms.

One of the proposed mechanisms for this correlation between alate production and mushroom formation is that termites normally suppress mushroom formation through consumption of primordia - the initial stages of mushroom formation (Batra and Batra 1967, Korb and Aanen 2003, Aanen 2006, Aanen and Boomsma 2006). Based on this general hypothesis there is one, often used version in which the typical asexual structures that are found in all species of Termitomyces - nodules - are also the primordia of mushrooms. In this thesis we refuted this version of the hypothesis by showing that asexual nodules are different from true primordia from the earliest developmental stages that could be sampled (chapter 2). However, our findings do not refute the more general hypothesis that termites suppress mushroom formation through consumption of primordia.

Re-reading older literature with the knowledge that nodules and primordia are two different structures, gives some insight. Dixon reported mushroom primordia in Odontotermes colonies without implying any relation to nodules - or bromatia as he called them (Dixon 1983). He did not systematically compare nodules to mushroom primordia, yet he found that in the dry season (when he observed no fructification) a small portion of the combs were bearing primordia. In his discussion he mentioned that the combs bearing primordia were not actively torn down by termites, but that after a mushroom appeared from a comb the remaining aborted primordia and the comb itself were ac- 
tively reduced by the termites. These findings indicate that consumption of primordia is not the way by which mushroom formation is controlled. Yet, behavioural experiments with termites and combs bearing mushroom primordia and combs with asexual nodules should be performed to give more conclusive answers. However, if consumption of primordia is not the way by which mushroom formation is suppressed, mushroom formation should be suppressed in a different way. As primordia are not found in most of the termite colonies at all (chapter 2) it is likely that their formation itself is suppressed. Then two questions remain, (i) what is the mechanism of primordia suppression by the termite host, and (ii) what are the mechanisms of mushroom formation and its relationship with termite control of reproduction?
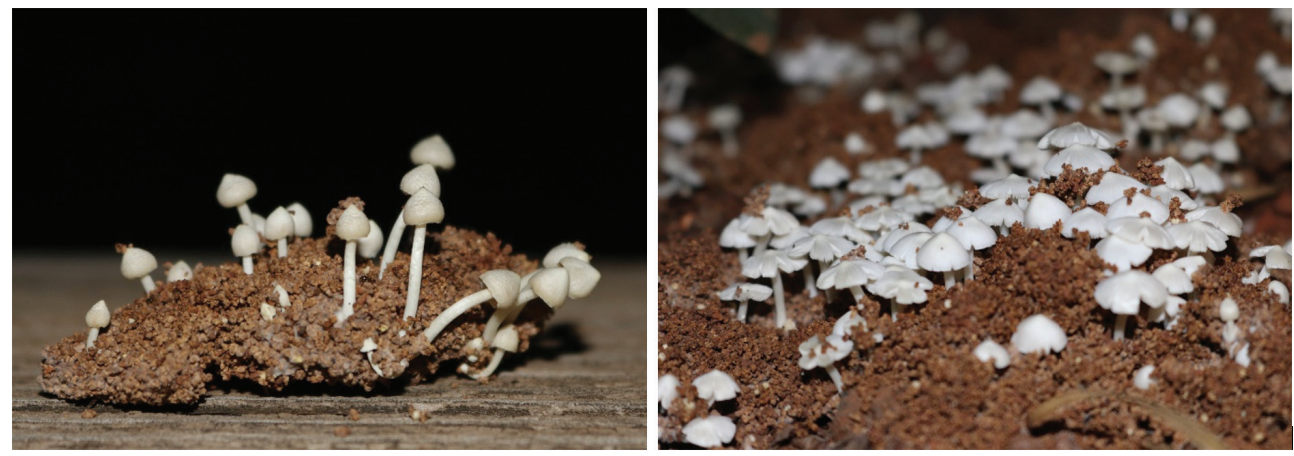

Figure 3| Termitomyces microcarpus mushrooms appear on fungus combs that are ejected from some Odontotermes colonies shortly after rainfall.

\section{An alternative hypothesis}

In addition to the observed coupling between termite reproduction and Termitomyces reproduction, there are other indications that termites control mushroom formation of their fungi. For example, one fungus, T. microcarpus, that is cultivated by some species of Odontotermes, forms mushrooms on fungal combs that are ejected from the colony; the ejected combs are no longer tended to by termites (Figure 3; Sieber 1983, Darlington 1994). Also, in species that have evolved vertical transmission, no mushrooms are found on top of the colonies (Johnson et al. 1981, Darlington 1994, Kone et al. 2011). Yet, the population structure of the vertically transmitted symbiont of $M$. bellicosus shows that occasional recombination, viæ. mushroom formation, takes place (Nobre et al. 2011a). Nobre et al. (2011a) found that the symbiont species of $M$. bellicosus is also cultivated by $M$. subbyalinus that employs horizontal transmission. These findings imply that $M$. bellicosus suppresses mushroom formation and $M$. subbyalinus seasonally allows mushroom formation. It is likely that $M$. bellicosus hosts occasionally pick up spores that are produced by mushrooms of a $M$. subbyalinus symbiont. Affirming these findings, Termitomyces mushrooms were observed on dead termite $M$. bellicosus colonies; colonies in 
which the fungus gardens are no longer tended to by termites and suppression is lifted (Nobre et al. 2011a).

A common feature to the induction of mushroom formation in other basidiomycetes is a sudden arrest of mycelial growth. This arrest can be due to changes in environmental or physiological factors, such as light, temperature, the availability of nutrients, injury, and gasses (oxygen and carbon dioxide) (reviewed by Kües and Liu 2000, Halbwachs et al. 2016, Sakamoto 2018). Mushroom formation is thought to be a response to escape an environment that is no longer suitable for vegetative growth. Nutrient limitation is one of the factors that can, under the appropriate environmental conditions such as humidity and temperature, induce mushroom formation. For this reason, in human cultivation of Agaricus bisporus - the button mushroom - compost that is colonised by fungal hyphae is covered with a nutrient poor casing layer (Kües and Liu 2000).

When we first observed mushroom primordia on fungal combs in a M. natalensis colony, we noticed that the comb fragments containing these primordia seemed to be older fungus comb. An indication for this is the colour of the fungus comb. Because Termitomyces is a white rot fungus - which means it breaks down lignin - the older the substrate is, the lighter it becomes by the breaking down of dark lignin (Figure 4; Hyodo et al. 2000, da Costa et al. 2018). Additionally, we observed the formation of primordia on a comb fragment that had been incubated for 16 days and had previously only borne asexual nodules. Combining these observations with the knowledge that mushroom formation can be a starvation response, and that combs that are ejected from the nest (in the species T. microcarpus) or are unattended in dead colonies start producing mushrooms, I hypothesise that Termitomyces mushrooms are found on fungus combs that are nutritionally depleted, because there is no new substrate added to these fungal combs.

In addition to explaining the observations of Microcarpus mushrooms on expelled combs and Termitomyces mushrooms on dying termite colonies, my hypothesis possibly also explains the observed co-occurrence of termite reproduction and mushroom formation. The production of alates likely leads to a temporary

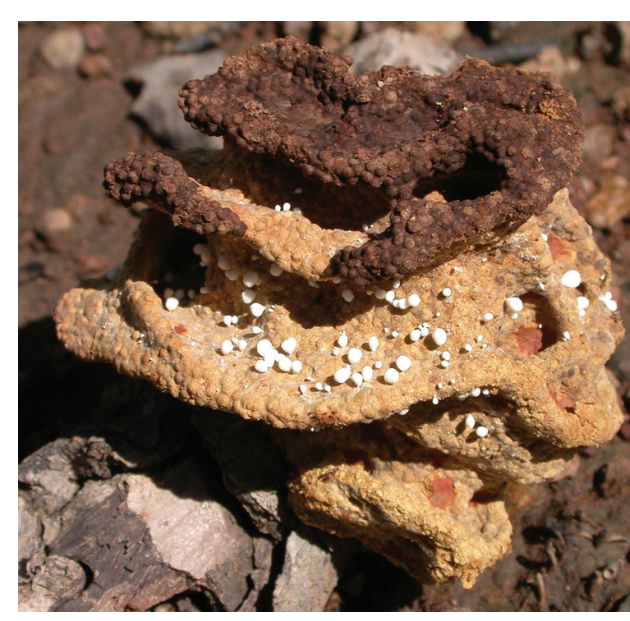

Figure 4| Fungus comb of Macrotermes bellicosus. The lighter comb on the bottom is older comb, in which more plant substrate has been broken down and more fungal hyphae have grown. The darker comb on top is fresh, just inoculated substrate. Photo credit: Judith Korb 
change in colony dynamics, because the colony's resources needed for alate production cannot be allocated to worker production. It could be that this leads to abandonment of parts of the fungus comb; in abandoned combs substrate is no longer added nor consumed. As a result, these combs become nutritionally depleted and under the right conditions (e.g. right humidity after rainfall) mushrooms can appear. Consistent with my hypothesis, abandoned combs have been observed in Macrotermes colonies, and not in Microtermes colonies (with vertical symbiont transmission) in which fungal combs are almost completely metabolised (Wood and Johnson 1978, Wood and Thomas 1989). However, especially in the context of the division of labour that is observed between different castes and age groups in Macrotermes and Odontotermes species that were studied, the exact colony dynamics that could lead to fungus comb abandonment are complex (Badertscher et al. 1983, Gerber et al. 1988, Hinze and Leuthold 1999, Hinze et al. 2002, Gladfelter and Berman 2009, Li et al. 2016). With the artificial cultivation system recently set-up by $\mathrm{Li}$ et al. (2016), it would be possible to study fungus comb turnover in more detail and test whether colony dynamics can lead to fungus comb neglection and thereby mushroom formation.

Analogously, in the convergently evolved obligate mutualistic symbiosis between attine ants and a different group of basidiomycete fungi (Order: Agaricales, tribe: Leucocoprineae), mushroom formation is also suppressed by the insect farmers (Mueller 2002). Recently, Shik et al. (2016) found that one of the mechanisms by which mushroom formation is controlled by the ants is by carefully managing the protein content in the substrate. If the fungus grows on a substrate that is too rich in protein, vegetative growth is hampered. However, if the fungus grows on a substrate that is depleted in protein it will start producing mushrooms (Shik et al. 2016). In their foraging behaviour, the ants manage protein provisioning to stay within the range in which hyphal production is good, but mushroom formation is not induced. To see whether the termites could have adapted a similar foraging strategy, we performed the same geometrical growth experiments for Termitomyces isolates (chapter 3; da Costa et al. 2019). We also found reduced vegetative growth on high protein substrates but did not find mushroom formation on substrates low in protein content. However, these experiments were carried out on Petri dishes, which removes the influence of the three-dimensional structure of the fungus comb. One of the effects of the three-dimensional structure of the fungus comb could be the regulation of air flow, and thereby the regulation of fungus' contact with gasses like oxygen and carbon dioxide. Also, the substrates contained simple carbohydrate and protein sources (glucose, starch and Bacto peptone and Trypticase peptone), which does not reflect the natural substrate of complex plant material (Figure 5). Observations of termite foraging behaviour in addition to experiments on more realistic growth substrates, could be performed in future to further explore the possibility that substrate provisioning by the termites suppresses mushroom formation in Termitomyces fungi. 


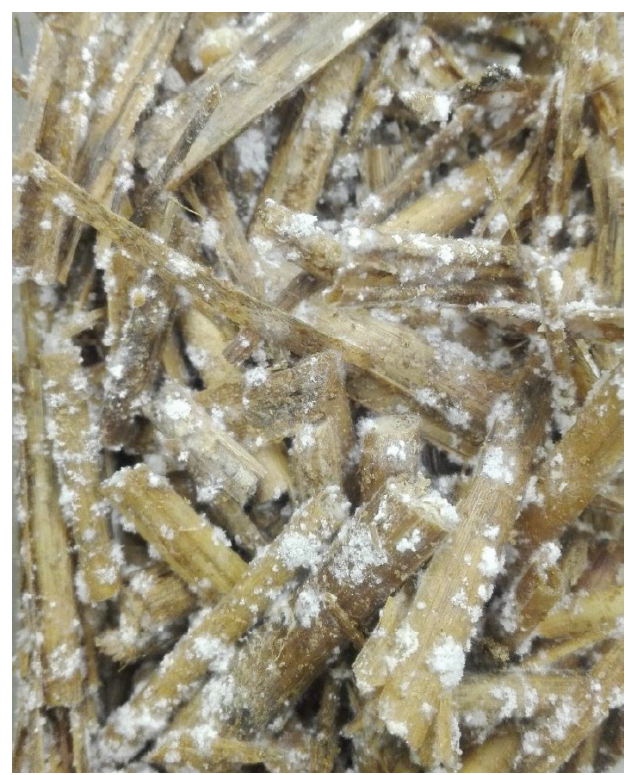

Figure 5| The Termitomyces symbiont of $M$. natalensis grown on hydrated Miscanthus (a grass) and 10\% milled wheat bran. A student I supervised has made the first steps towards culturing Termitomyces on more realistic growth substrates. His main finding was that inoculation via brood leads to better results than inoculation via spore suspension, because it leads to faster colonisation and less contamination of the substrate. Also, he tested multiple carriers and found that Miscanthus as a carrier gave the best results. He hypothesised that this was due to the airy structure of the substrate and because it retained relatively little water (Gaertner 2019).

\section{What is control?}

The term "control" has the connotation that the termites consciously make the decision to control the fruiting of their fungus, which is obviously not the case. However, if my hypothesis about the induction of primordia through neglect of fungal combs is true, this does mean that the termites control fungal reproduction, in the sense that their behaviour affects whether the fungus does or does not fruit. Still the question remains whether this termite behaviour has been selected for because control over fungal fruiting provided a selective benefit, or whether the control over fungal fruiting is a by-product of termite behaviour that evolved in a different way? How does this compare to the seemingly active control of fungal reproduction by the fungus-growing ants?

Both the termites and the ants select fungal cultivars on vegetative production; both termites and ants inoculate new parts on the fungus comb with vegetative fungal inoculums (asexual spores and mycelium respectively) (Leuthold et al. 1989, Darlington 1994, Mueller 2002, Aanen 2006). Fresh substrate is continuously

provided. When a fungus can continue to grow vegetatively it is less likely to reproduce sexually, which applies both to the ant-fungus as well as to the termite-fungus. In this sense suppression of fungal fruiting would be a by-product of selection for vegetative fungal growth. In addition, the conflict over symbiont mixing is resolved in both antsand termite-fungus symbiosis; in each colony only a single cultivar is maintained. The mechanisms that reduce symbiont mixing in the ant-fungus and in the termite-fungus symbiosis are, however, not reliant on the repression of fungal reproduction. In the fungus-growing termites a single heterokaryon can be quickly selected from multiple different starter cultures trough frequency dependent selection (Aanen et al. 2009), and in the fungus-growing ants each new colony is inoculated with a single strain by the 
founding queen (Mueller 2002). In conclusion, I think that in both symbioses fruiting suppression can be explained by selection of termite/fungus behaviour that provided a selective benefit in itself and that as a by-product, may have resulted in repression of fungal fruiting.

\section{On the role of nodules in the origin of termite-fungus symbiosis}

The finding that asexual nodules are not the primordia of mushrooms also has an important implication for another hypothesis that leans on the assumption that nodules are primordia: asexual spores that are formed by all studied Termitomyces species are an adaption to termite consumption of immature mushrooms (Aanen 2006, Aanen and Boomsma 2006, Nobre et al. 2011c). Our findings suggest that this hypothesis can be rejected, although I think that nodules played an important role in the origin of the termite-fungus symbiosis.

Recently, Bucek et al. (2019) presented evidence that the ancestor of the fungus-growing termites was already building faecal combs without growing fungi. They showed that the comb-building family Sphaerotermitinae is the sister group of the comb-building Macrotermitinae. The single species of the Sphaerotermitinae cultivates bacteria rather than fungi on its combs (Garnier-Sillam et al. 1989). The most parsimonious reconstruction is that the ancestor of all Termitomyces fungi started to grow on combs that were already present in the ancestor of all fungus-growing termites. Many wood-feeding (non-fungus-growing) termites are attracted to rotting wood and foraging on fungus-infested wood increases termite survivorship substantially (Viana-Junior et al. 2018). Therefore, the Termitomyces ancestor probably was not the only fungus that infested the termite comb, but it may have been the only fungus that found a way to escape destruction by the termite gut (Leuthold et al. 1989). Thomas was the first to show that indeed the first gut passage dramatically reduced the number of culturable fungal units that were previously present in the foraged material (Thomas 1987a). Moreover, none of the fungi that she found in the foraged material were Termitomyces species. Later Leuthold et al. (1989) showed that only the Termitomyces asexual spores could survive termite gut passage, whereas Termitomyces hyphae could not.

Following from the observation that only the asexual conidiospores and not fungal hyphae can pass the termite gut unharmed, and from the fact that all studied Termitomyces species make these asexual conidiospores, it is likely that the asexual spores play a crucial role in the origin of the termite-fungus symbiosis (Aanen 2006). Interestingly, recent work in our lab showed that Artbromyces fungi, found in Central America, are a sister group to Termitomyces fungi and that at least one Arthromyces species produces asexual spores on vegetative mycelium in a similar fashion to Termitomyces (Botha and Eicker 
1991, Baroni et al. 2007, van de Peppel et al. submitted). This suggests that asexual spores were already present in the last common ancestor of Termitomyces and Arthromyces, especially since asexual spores are relatively rare (Kües et al. 2016, van de Peppel et al. submitted). Also, to find out whether the gut resistance of Termitomyces spores is an adaption to the symbiosis, it would be very interesting to study whether the asexual spores of Artbromyces fungi can survive termite gut passage.

Regardless of whether asexual Termitomyces spores were already present before they were domesticated by termites, it is highly likely that the nodules as they are found now in Termitomyces have been adapted to domestication. A comparison of symbionts of Macrotermes natalensis and Odontotermes badius showed that the microscopic characters of the spores are more similar - vir; in size, branching pattern and ontogeny - than the nodules on fungal combs as a whole (Botha and Eicker 1991). Also, laboratory studies showed that Termitomyces fungi have slow radial growth and are easily contaminated by other fungi (Petch 1906, Darlington 1994, Ono et al. 2017). Through harvesting of asexual spores and regular and fast destruction of vegetative mycelia, Termitomyces fungi have been selected to invest in the production of asexual spores, rather than mycelial growth, in contrast to most free-living basidiomycete fungi (Nobre et al. 2011c). The more asexual spores a strain of Termitomyces produces, the more it is inoculated in new parts of the fungus comb, and the higher the chance one mycelial patch can fuse to a genetically identical mycelial patch, which has been shown to increase spore production (Aanen et al. 2009). It is likely that this alteration in the mode of fungal colonisation made Termitomyces fungi dependent on their termite hosts. As a by-product only one heterokaryon remains in each termite colony, removing possible detrimental competition between symbionts, despite multiple symbionts possibly inoculating each newly founded colony (Aanen 2006, Aanen et al. 2009). The resulting monoculture removes harmful competitive interactions between genetically different heterokaryons, thus aligning the interests of fungus with those of the termite colony.

\section{How is the right fungus selected?}

In fungus-growing termites large differences in interaction specificity are observed that do not necessarily correlate with the mode of transmission (Aanen et al. 2002, Aanen et al. 2007, Nobre et al. 2011b, Nobre and Aanen 2012, van de Peppel and Aanen 2020). For example, the termite species Macrotermes natalensis, which employs a horizontal mode of transmission is only found to cultivate one lineage of Termitomyces (De Fine Licht et al. 2006, Aanen et al. 2007), which has been found to represent a single biological species (Nobre et al., 2014). On the other hand, termites of the genus Microtermes and of the species $M$. bellicosus - both vertically transmitted - are found to cultivate multiple fungal genotypes. Despite these differences there is a certain degree of specificity between 
the fungus-growing termites and their fungi at the level of the termite genus; species from a single termite genus or in some cases closely related genera generally cultivate fungi from the corresponding monophyletic group of Termitomyces fungi (Aanen et al. 2002, Rouland-Lefèvre and Bignell 2002, Aanen et al. 2007).

One of the often-proposed causes for the observed interaction specificity and co-evolution is nutritional interdependence; one of the partners loses the ability to metabolise a certain substrate, because its partner reliably provides it, and vice versa (Visser et al. 2010, Ellers et al. 2012). In chapter 3 we tested one part of this hypothesis, vi\% whether different Termitomyces isolate have different growth capacities on 1) different carbohydrate substrates and 2) different ratios of protein and carbohydrate in a two-factor nutritional geometric framework experiment. We tested this for the symbiont of $M$. natalensis (high interaction specificity) and two isolates of Odontotermes symbionts (intermediate interaction specificity). Following from the observed co-evolution it was expected that the Odontotermes symbionts would have a different growth profile than the M. natalensis symbionts. Also, following from the observed interaction specificity between Odontotermes species and their symbionts, it was expected that their growth patterns would be more similar to each other than to the $M$. natalensis symbiont as they are presumed to be more generalist than the M. natalensis symbiont (Rouland-Lefèvre 2000, De Fine Licht et al. 2007).

In the discussion of chapter $\mathbf{3}$ we hypothesised that all Termitomyces isolates that we studied formed more biomass on cellobiose than on D-glucose because we standardised carbon-source concentrations rather than carbon content. Due to this, media with cellobiose contained double the amount of carbon compared to the media that contained $\mathrm{D}$-glucose as a C-source. We tested this hypothesis by comparing growth on media with cellobiose and D-glucose that were standardised on carbon content and indeed found similar growth on both media, which indicates that our hypothesis was correct (Coolen 2018).

The findings in chapter 3 indicate that the Termitomyces symbionts cultivated by the three species could to some extent be adapted to different substrates. However, our experiments were one-sided in the sense that they only assessed the ability of biomass formation and radial growth of Termitomyces symbionts in an artificial setup. Substrate breakdown is not only achieved by Termitomyces in this symbiosis. The termites and their gut microbiota also play a role in the breakdown of plant material and different degrees of complementarity may have evolved between the partners of the symbiosis (Nobre and Aanen 2012, Poulsen et al. 2014). For M. natalensis it was shown that the genetic potential of carbohydrate substrate breakdown in the termite, its symbiont and the termite gut microbiota showed complementarity; the microbiome was shown to target simpler sugars while the Termitomyces symbiont lacked some of the genes to use simple 
sugars (Poulsen et al. 2014). A recent study in our lab that compared the CAZyme genes (carbohydrate active enzyme genes) in four different Termitomyces isolates studies did not find any striking differences between the Termitomyces species (van de Peppel et al. submitted). This is consistent with what we found in chapter 3: most differences were quantitative rather than qualitative, indicating that possible differences could also be explained by differences in expression levels or enzymes efficiencies.

In chapter 4 we constructed a more contiguous version of the Termitomyces reference assembly accompanied by a genetic linkage map. Both could help in future studies of the metabolic capacity of different Termitomyces strains. With the help of these results the breakdown capacity of different substrates could be analysed using Quantitative Trait Analysis (QTL). Also, it is likely to provide more information on the regulatory parts of the genome that are often not captured in a less contiguous genome. Finally, a more contiguous genome will aid the analysis of gene clusters, which may play an important role in metabolic pathways (Slot and Hibbett 2007, Thomma et al. 2016).

\section{The possible role of nitrogen in interaction specificity}

Termites (non-fungus-growing) are known to store uric acid - a final product of nitrogen metabolism in termites - in the fat body (Potrikus and Breznak 1981, Slaytor and Chappell 1994). They can, however, not mobilise this uric acid because they lack the enzymes to do this. It is thought that the uric acid is recycled in the colony through necrophagy and cannibalism, and broken down by gut bacteria capable of uric acid degradation (Potrikus and Breznak 1980, Slaytor and Chappell 1994). It is not completely clear whether fungus-growing termites also store uric acid, and this has only been studied in a few Macrotermes species (Tayasu et al. 2002). The data of Tayasu et al. indicate that in some Macrotermes species older workers may retain uric acid (Tayasu et al. 2002). The latter would indicate that nitrogen is preserved in the colony if these older workers are eaten by their younger conspecifics. The absence of accumulation in younger workers, or (in some colonies) in all sampled workers could indicate that uric acid is deposited in the fungus comb and utilised by Termitomyces as a reliable nitrogen source.

Nitrogen is required by all organisms, it is needed for the production of proteins and nucleic acids - the building blocks of proteins, DNA and RNA - among other things (Caddick 2004). In general fungi live in habitats where the availability of nutrients can be unpredictable. Most fungi are therefore selected to maintain diverse metabolic capacities (Wong et al. 2008). These metabolic pathways are, however, selectively expressed. The energetically more 'expensive' pathways are suppressed by the presence of energetically 'cheaper' nitrogen sources, such as ammonium and glutamine (Caddick 2004, Wong et al. 2008). For Termitomyces the environment in which it grows and the nutrition 
it receives is much more predictable than for free-living fungi. It is therefore not unlikely that certain metabolic pathways, such as the energetically costly pathway to breakdown nitrate, can deteriorate over time as there is no selective pressure to maintain them (Caddick 2004).

In addition to growth ability on different carbon sources, three students that I supervised studied nitrogen utilisation in Termitomyces isolates (Runderkamp 2016, Coolen 2018, Hensen 2018). Their most striking finding was that some Termitomyces isolates, amongst which all $M$. natalensis symbionts, some Odontotermes symbionts and some Microtermes symbionts, were unable to grow on sodium nitrate, whereas others, some Odontotermes symbionts and some Microtermes symbionts were. Also, on average the highest growth was obtained on urea (Runderkamp 2016, Coolen 2018, Hensen 2018). Urea is a breakdown product of, amongst others, uric acid which is excreted by most birds, reptiles and terrestrial insects (Geisseler et al. 2010). In subsequent experiments it would be interesting to study whether different Termitomyces species can grow on uric acid as a sole nitrogen source. Finally, future research should aim at comparing the ability to grow on different nitrogen sources of Termitomyces with their phylogeny, to see whether possible losses or gains of nitrogen assimilation pathways occurred independently or have a common origin. Also, it should be studied by which termites these isolates are cultivated, to see whether nitrogen metabolism could be an adaptation to the termite symbiosis that enhances interaction specificity.

\section{Other possible contributors to interaction specificity}

In this thesis I have mainly considered nutritional aspects, or complementarity as a possible underlying cause of the observed interaction specificity. However, there are also other factors that could contribute. The first contributor could be timing of the founding of new nests and the appearance of mushrooms in mounds of conspecifics. It is known that symbionts of different termite species fruit at different times, which could make sexual spores of the most coevolved symbiont more abundant around the time that a new colony needs to be inoculated (Kone et al. 2011). A second contributor could be the role of the first gut passage. During this gut passage the substrate is physically disrupted (masticated), saliva is added, the fungus is inoculated at a certain density, and enzymes are added (Nobre and Aanen 2012, da Costa et al. 2018). Different termite species may work their substrate in slightly different manners creating optimal growth conditions for their co-evolved species, which can then form most sexual spores and is selected (Aanen et al. 2009). A third contributor could be the construction of the comb on which the symbiont is cultivated. Different fungus-growing termite species construct their fungus combs differently, which may affect a sexual spore-yield and thus favour a better adapted cultivar. 


\section{The life cycle of Termitomyces compared to other basidiomycetes}

Until now I have mainly discussed mechanisms that mitigate conflicts of interest between host and symbiont in the termite-fungus mutualism; conflicts between selection at the fungus level and cooperation at the mutualism level. In chapter $\mathbf{5}$, however, we zoomed in on possible conflicts that can arise within the organisational level of a basidiomycete individual as a consequence of the basidiomycete life cycle (Vreeburg et al. 2016). Here I would like to extend the ideas of chapter 5 to the Termitomyces life cycle, that differs from the 'standard' basidiomycete life cycle in several ways.

I will start here by repeating the 'standard' basidiomycete life cycle that is mainly based on the life cycle of Schizophyllum commune (also see chapter 5, Figure 1). Haploid sexual spores germinate to form a monokaryon, a connected hyphal network in which each cell contains a single haploid nucleus. The monokaryon can grow only vegetatively. Two monokaryons can fuse and if the nuclei of the respective monokaryons are compatible in the sense that they have different alleles at the two mating type loci - the homeodomain (HD) locus and the pheromone receptor (PR) locus -, a developmental program is triggered that leads to formation of a dikaryon. The nuclei of the former monokaryons migrate through the mycelium of their fusion partner and once the cells at the growing tips of the mycelium have become dikaryotic, the two different nuclei start synchronous division. New cells are formed using clamp connections that ensure that each new cell contains exactly one copy of both nuclei. The dikaryon can grow vegetatively, but also sexually through the formation of mushrooms in which haploid sexual spores are produced by meiosis after a short diploid phase in specialised cells called basidia.

The first difference between Termitomyces - at least the species that is associated with M. natalensis - and the 'standard' basidiomycete life cycle is the absence of nuclear migration in Termitomyces (chapter 4; Nobre et al. 2014). This means that, in contrast to what we discuss in chapter 5 , in a mating between $M$. natalensis symbionts, none of the homokaryons take the female role in the sense that the whole mycelium is fertilised. The homokaryons that mate are thus not exposed to the risks of the female role. Also, Nobre et al. (2014) argued that the lack of nuclear migration may even be the result of reduced selection on the nuclear level. Nuclear migration may be lost, because the way in which the Termitomyces is cultivated - through repeated inoculation of asexual spores - hardly leaves the opportunity for fast radial growth and nuclear migration. Thus, the mechanism of repeated inoculation would have mitigated one opportunity for competition at the nuclear level that could possibly have negative effects at the mycelial level. However, at the same time the absence of nuclear migration does make the possibility for genomic conflict due to mitochondrial competition a real threat. 
In the 'standard' basidiomycete life cycle, there is only a small part of the mated mycelium that contains two types of mitochondria. Only the interaction zone of a fused cell contains both mitochondria, but the rest of the mycelium contains only one type of mitochondria, from the previously 'female' homokaryon. In the absence of nuclear migration, however, the resulting heterokaryon will contain two different types of mitochondria, because the interaction zone gives rise to the whole heterokaryon. This bi-parental mode of mitochondrial transmission can induce competition between the different mitochondria at the cost of the mycelium and thus at the cost of the higher organisational level. Yet, in a study of the mitochondrial DNA of 12 Termitomyces symbionts from termites of three different genera (Macrotermes, Odontotermes and Microtermes), only one type of mitochondrial DNA was found for each isolate, which implies that there is a mechanism by which only one mitochondrial type remains in the colony's heterokaryon (Nieuwenhuis et al. 2019). The latter is in line with the fact that in nature hardly any organism is found with multiple mitochondrial types (Basse 2010), although heteroplasmy in cells close to the interaction zone between two homokaryons has been found in multiple basidiomycete species (May and Taylor 1988, Matsumoto and Fukumasa-Nakai 1996, Barroso and Labarère 1997).

Whether in the whole $M$. natalensis termite colony only one type of mitochondria is maintained remains to be tested. If this is indeed the case, it can be studied how one mitochondrial type is retained in each fungus colony. It would be very interesting to try to obtain a heteroplasmic $M$. natalensis symbiont and study whether this heteroplasmy is maintained and whether it negatively effects heterokaryon. Finally, it should be studied whether the absence of nuclear migration is also observed in other species of Termitomyces.

Another clear deviation from the standard basidiomycete life cycle is that most studied Termitomyces species lack clamp-connections (Bi et al. 1993, De Fine Licht et al. 2005). Consequently, the number of nuclei within a cell is not as strictly regulated as in a dikaryotic fungus. In the Termitomyces symbiont of $M$. natalensis up to 10 nuclei per cell have been found (De Fine Licht et al. 2005). In theory this could lead to competition between the nuclei and thereby to nucleus level selection that is in conflict with mycelium level organisation. Especially since Termitomyces fungi produce asexual spores - another aberration from the 'standard' life cycle - it could be that the nuclei are selected to preferentially position themselves in the spores and thereby have an advantage in the next inoculation. However, Nobre et al. (2014) showed that in reality, escape from the heterokaryon through the formation of homokaryotic spores is not observed. In addition, they showed that heterokaryons invariably produced more spores than homokaryons, which means that even if a homokaryotic spore is produced it is likely quickly outcompeted by the heterokaryotic spores upon inoculation in the fungus comb. 
To gain more insight into the life cycle of Termitomyces, a student that I supervised attempted to establish a genetic Agrobacterium-mediated transformation protocol to introduce histones labelled with a fluorescent tail (Packbier 2018). In other filamentous fungi this visualisation tool has allowed researchers to study nuclear dynamics and hyphal fusion between differently labelled strains (Freitag et al. 2004, Rech et al. 2007). I would suggest continuing Packbier's work in future, to show microscopically what happens after fusion of two homokaryons, whether each cell in the heterokaryons indeed contains the two constituent nuclei and if so, how this is maintained, .

\section{Is there a 'standard' basidiomycete life cycle?}

Clearly the life cycle of Termitomyces fungi differs from the 'standard' basidiomycete life cycle in some key respects. However, it is certainly not the only basidiomycete that deviates. As stated before, it is relatively rare for a mushroom forming basidiomycete to also form asexual spores, although the myriad of ways by which different basidiomycetes do form asexual spores shows that there are many deviations from the 'standard' life cycle. Also, a lack of clamp connections is not unique. The well-known button mushroom Agaricus bisporus - also lacks clamp connections (Raper et al. 1972). The same species also does not have nuclear migration (Raper et al. 1972, Xu et al. 1993). Another species that generally lacks clamp connections is the fungus Lencoagaricus gongylophorus, the symbiont of the leaf-cutter ant (Vellinga et al. 2003). However, symbionts of the leaf-cutter ants are not only multinucleate, but probably also polynucleate, in the sense that they contain more than two different nuclei in one mycelium (Kooij et al. 2015). Not even the 'living apart together' of two separate nuclei within one mycelium is standard. The mushroom forming basidiomycete Armillaria mellea has an extended diploid stage in which it grows vegetatively (Ullrich and Anderson 1978).

Many deviations from the 'standard' life cycle of basidiomycetes can thus be found. Yet, we have studied only a fraction of the basidiomycetes that exist. In that respect we are living in an exciting era, where more and more organisms can be studied that are not a 'model' in one way or the other. As I showed in chapter 4, even constructing a species' reference genome can now be done by a handful of scientists (and some powerful computers and algorithms). I predict that we will find a plethora of deviations from the 'standard' basidiomycete life cycle that will make us reconsider whether there even is such a thing as the 'standard' basidiomycete life cycle. It would be very interesting to study and compare all these life cycle differences as many of their peculiarities will be answers to the question posed by Maynard Smith and Szathmáry: "Why not did natural selection, acting on entities at the lower level ..., disrupt integration at the higher level?" (Maynard Smith and Szathmáry 1995). Buss (1987) already argued that observed life cycles are the result of selection on life cycles that can resolve conflict between the different levels of 
selection. I think that we will be able to categorise Basidiomycete life cycles according to their mechanisms of resolving the conflict between lower-level selection on the individual nuclei and mitochondria, and the higher-level organisation of the fungal individual. Thereby we will be able to paint a clearer picture of the extent to which theoretical conflicts play an actual role in the evolution of life cycles.

\section{Towards re-domesticating Termitomyces mushrooms}

The original title of the proposal for this thesis was: "Re-domesticating an ancient domesticated fungus". The core idea of the project was to find out how we, human farmers, could cultivate the protein-rich, highly prized Termitomyces mushrooms for our own consumption. I have often explained my project, especially chapter 2 , to other people from this perspective, as it is 1.) more accessible to people who do not have a background in evolutionary biology, and 2.) it seems to have a more direct relevance, i.e. it can aid the protein transition - moving our diets away from excess animal protein consumption. I purposely say "seems to have more direct relevance", because understanding how higher levels of organisation - such as mutualisms - remain stable is very relevant for our understanding the biological world and its hierarchical organisation. Nevertheless, all the work I have done on primordia of mushroom formation (chapter 2), the growth requirements of different Termitomyces strains (chapter 3 ), and the genome and linkage map (chapter 4) are all relevant for re-domestication of Termitomyces mushrooms.

Being able to cultivate Termitomyces mushrooms is something that has often been tried, but so far with no success. The interest in cultivation methods is two-sided: 1.) for research purposes, being able to complete the sexual cycle would open many possibilities, 2.) for consumption; Termitomyces mushrooms are highly prized and likely have a large market potential. One study showed mushroom formation in laboratory settings of Termitomyces microcarpus, but we did not manage to replicate the findings (De 1983). Reports have been made of Termitomyces mushrooms that were commercially cultivated, although others have noted that at least one widely cultivated cultivar that is presumed to be a Termitomyces mushroom called "Black Termite Mushroom", actually belongs to the species Oudemanciella raphanipes (Hao et al. 2016). Some patents claiming Termitomyces mushroom cultivation techniques have also been published, but in the light of the work of Hao et al. (2016) it seems doubtful that these are indeed Termitomyces species.

In reaction to talks about the possibilities of Termitomyces mushroom domestication, I would often be asked the question whether Termitomyces mushrooms are a good meat replacement. According to the Dutch "Voedingscentrum" - the Dutch organisation that is responsible for providing accessible, scientifically sound, advise to consumers about healthy, safe and sustainable nutrition - meat replacement needs to contain sufficient 
protein, iron, vitamins B1 and B12 (Voedingscentrum 2020). In that sense no mushroom is a good meat replacement. However, even though I will not deny that these four nutrients are important, I do think that this view is very 'meat-centred', and not the way in which I think we should approach our nutrition.

It is very clear that both from a human health perspective as well as from a planet health perspective a dietary shift needs to occur. We need to greatly increase consumption of plant-based products and at the same time greatly reduce the intake of animal products to reduce pressure on the environment and improve health outcomes. The recent publication by the EAT-lancet Commission, shows the importance of a varied, mostly plant-based diet (Willett et al. 2019). After an extensive review of all reliable available evidence, the commission concludes that the following dietary patterns promote low risk of major chronic disease and overall well-being (Willett et al. 2019):

1. protein sources primarily from plants, including soy foods, other legumes, and nuts, fish or alternative sources of omega-3 fatty acids several times per week with optional modest consumption of poultry and eggs, and low intakes of red meat, if any, especially processed meat;

2. fat mostly from unsaturated plant sources, with low intakes of saturated fats, and no partly hydrogenated oils;

3. carbohydrates primarily from whole grains with low intake of refined grains and less than $5 \%$ of energy from sugar;

4. at least five servings of fruits and vegetables per day, not including potatoes;

5. moderate dairy consumption as an option.

In short, meat does not necessarily need to be replaced in a varied diet. Vitamin B12, a vitamin that is only produced by bacteria and stored in animal tissue, is the only nutrient that needs to be supplemented, but this can be done via supplements.

One thing that strikes me both in the study of the EAT-lancet commission as well as in "de schijf van vijf" - the dietary advice from the "Voedingscentrum", is that mushrooms are not mentioned in any of the categories. Also, in my discussions with friends and family I would notice that it is hard to place mushrooms in a category; if I was talking about fungi, they would classify them as microorganism, whereas if I was talking about edible mushrooms they would classify them as vegetables. Yet, mushrooms contain nutrients that can be found in plants and animals, but the blend is unique to the fungal kingdom (Feeney et al. 2014). Compared to cultivation of plants, mushroom cultivation and especially breeding is still largely unexplored. For example, the common button mushroom was bred almost 40 years ago and has not been much improved upon since then (Fritsche 1986, Sonnenberg et al. 2011). 
So, what would be my answer to the question "are Termitomyces mushrooms a good meat replacement"? Simple, you do not need a meat replacement, you need a balanced diet in which the nutrients you need are provided and with which you do not deplete the earth's resources. Termitomyces mushrooms are rich in protein, contain essential amino acids and mushrooms in general are a source of dietary fibre, mineral and vitamins. Finally, mushrooms including Termitomyces can grow on recalcitrant woody materials that could not be directly consumed by people. In conclusion Termitomyces mushrooms can be part of a healthy, sustainable diet. On top of this they are delicious as prof. Bernard Slippers reminisced: "The taste is not easy to describe - it is delicious, that is for sure. People say it has a meaty taste - but not quite like meat - much softer, and rich but not fatty. And with a texture that is firm but melt-in-your mouth mushroom-like." 
Aanen, D. K., P. Eggleton, C. Rouland-Lefevre, T. Guldberg-Froslev, S. Rosendahl, et al. (2002). "The evolution of fungus-growing termites and their mutualistic fungal symbionts." Proceedings of the National Academy of Sciences of the United States of America 99(23): 14887-14892.

Aanen, D. K., T. W. Kuyper, A. J. Debets and R. F. Hoekstra (2004). "The evolution of non-reciprocal nuclear exchange in mushrooms as a consequence of genomic conflict." Proc Biol Sci 271(1545): 1235-1241.

Aanen, D. K. and P. Eggleton (2005). "Fungus-growing termites originated in African rain forest." Current Biology 15(9): 851-855.

Aanen, D. K. (2006). "As you reap, so shall you sow: coupling of harvesting and inoculating stabilizes the mutualism between termites and fungi." Biology Letters 2(2): 209-212.

Aanen, D. K. and J. J. Boomsma (2006). The evolutionary origin and maintenance of the mutualistic symbiosis between termites and fungi. Insect Symbiosis, Volume 2. K. Bourtzis and T. A. Miller, CRC press: 79-95.

Aanen, D. K. and R. F. Hoekstra (2007). Why sex is good: on fungi and beyond. Sex in fungi: molecular determination and evolutionary implications. J. Heitman, J. W. Kronstad, J. W. Taylor and L. A. Casselton. Washington DC, ASM press: 527-534.

Aanen, D. K., V. I. D. Ros, H. H. D. Licht, J. Mitchell, Z. W. de Beer, et al. (2007). "Patterns of interaction specificity of fungus-growing termites and Termitomyces symbionts in South Africa." Bmc Evolutionary Biology 7.

Aanen, D. K., H. H. De Fine Licht, A. J. M. Debets, N. A. G. Kerstes, R. F. Hoekstra, et al. (2009). "High Symbiont Relatedness Stabilizes Mutualistic Cooperation in Fungus-Growing Termites." Science 326(5956): 1103-1106.

Adejumo, T. and O. B. Awosanya (2005). "Proximate and mineral composition of four edible mushroom species from South Western Nigeria." African Journal of Biotechnology 4: 1084-1088.

Ainsworth, A. M., A. D. M. Rayner, S. J. Broxholme and J. R. Beeching (1990). "Occurrence of unilateral genetic transfer and genomic replacement between strains of Stereum hirsutum from non-outcrossing and outcrossing populations." New Phytologist 115(1): 119-128.

Aletor, V. A. (1995). "Compositional studies on edible tropical species of mushrooms." Food Chemistry 54(3): 265-268.

Alexander, I. (2018). Diagram of the six possible types of symbiotic relationship, from mutual benefit to mutual harm. Wikimedia Commons, licensed under the Creative Commons Attribution-Share Alike 4.0 International license.

Allaway, A. E. and D. H. Jennings (1970). "The influence of cations on glucose transport and metabolism by, and the loss of sugar alcohols from, the fungus Dendryphiella salina." New Phytologist 69(2): 581-593.

Altschul, S. F., W. Gish, W. Miller, E. W. Myers and D. J. Lipman (1990). "Basic local alignment search tool." J Mol Biol 215(3): 403-410.

Anderson, J. B. and L. M. Kohn (2007). Dikaryons, diploids, and evolution. Sex in fungi: molecular determination and evolutionary implications. J. Heitman, J. W. Kronstad, J. W. Taylor and L. A. Casselton. Washington, DC, ASM Press: 333-348.

Anderson, J. B. and S. Catona (2014). "Genomewide mutation dynamic within a long-lived individual of Armillaria gallica." Mycologia 106(4): 642-648.

Arita, I. (1979). "The mechanism of spontaneous dedikaryotization in hyphae of Pholiota nameko.” Mycologia 71: 603-611.

Arshad, M. A. and M. Schnitzer (1987). "The chemistry of a termite fungus comb." Plant and Soil 98(2): 247-256.

Ásgeirsdóttir, S. A., F. H. J. Schuren and J. G. H. Wessels (1994). "Assignment of genes to pulse-field separated chromosomes of Schizophyllum commune." Mycological Research 98(6): 689-693.

Badalyan, S. M., E. Polak, R. Hermann, M. Aebi and U. Kues (2004). "Role of peg formation in clamp cell fusion of homobasidiomycete fungi." J Basic Microbiol 44(3): 167-177. 
Badertscher, S., C. Gerber and R. H. Leuthold (1983). "Polyethism in Food-Supply and Processing in Termite Colonies of Macrotermes-Subhyalinus (Isoptera).” Behavioral Ecology and Sociobiology 12(2): 115-119.

Bankevich, A., S. Nurk, D. Antipov, A. A. Gurevich, M. Dvorkin, et al. (2012). "SPAdes: a new genome assembly algorithm and its applications to single-cell sequencing." J Comput Biol 19(5): 455-477.

Baroni, T. J., A. E. Franco-Molano, D. J. Lodge, D. L. Lindner, E. Horak, et al. (2007). "Arthromyces and Blastosporella, two new genera of conidia-producing lyophylloid agarics (Agaricales, Basidiomycota) from the neotropics." Mycological Research 111(5): 572-580.

Barroso, G. and J. Labarère (1997). "Genetic evidence for nonrandom sorting of mitochondria in the basidiomycete Agrocybe aegerita." Applied and environmental microbiology 63(12): 4686-4691.

Basse, C. W. (2010). "Mitochondrial inheritance in fungi." Current Opinion in Microbiology 13(6): 712-719.

Bastiaans, E., A. J. M. Debets and D. K. Aanen (2016). "Experimental evolution reveals that high relatedness protects multicellular cooperation from cheaters." Nature Communications 7.

Bathellier, J. (1927). Contribution à l'etude systématique et biologique de termites de l'Indo-Chine. . Société d'éditions géographiques, maritimes et coloniales. A. Gruvel, Société d'éditions géographiques, maritimes et coloniales. 1.

Batra, L. R. and S. W. T. Batra (1966). "Fungus-Growing Termites of Tropical India and Associated Fungi." Journal of the Kansas Entomological Society 39(4): 725-738.

Batra, S. W. T. and L. R. Batra (1967). "The Fungus Gardens of Insects." Scientific American 217(5): 112-124.

Benjamini, Y. and Y. Hochberg (1995). "Controlling the False Discovery Rate: A Practical and Powerful Approach to Multiple Testing." Journal of the Royal Statistical Society: Series B (Methodological) 57(1): 289-300.

Benoit, I., H. Culleton, M. Zhou, M. DiFalco, G. Aguilar-Osorio, et al. (2015). "Closely related fungi employ diverse enzymatic strategies to degrade plant biomass." Biotechnology for Biofuels 8(1): 107.

Bergstrom, C. T. and M. Lachmann (2003). "The Red King effect: When the slowest runner wins the coevolutionary race." Proceedings of the National Academy of Sciences 100(2): 593-598.

Bi, Z., G. Zheng and T. Li (1993). The macrofungus flora of China's Guangdong Province. Hong Kong, Chinese University Press.

Boddy, L. (1999). "Saprotrophic cord-forming fungi: meeting the challenge of heterogeneous environments." Mycologia 91(1): 13-32.

Bonner, J. T., K. K. Kane and R. H. Levey (1956). "Studies on the Mechanics of Growth in the Common Mushroom, Agaricus-Campestris." Mycologia 48(1): 13-19.

Boomsma, J. J. and R. Gawne (2018). "Superorganismality and caste differentiation as points of no return: how the major evolutionary transitions were lost in translation." Biological Reviews 93(1): 28-54.

Boswell, G. P., H. Jacobs, F. A. Davidson, G. M. Gadd and K. Ritz (2002). "Functional consequences of nutrient translocation in mycelial fungi." J Theor Biol 217(4): 459-477.

Botha, W. J. and A. Eicker (1991). "Cultural studies on the genus Termitomyces in South Africa. II. Macro- and micromorphology of comb sporodochia.” Mycological Research 95(4): 444-451.

Botha, W. J. and A. Eicker (1992). "Nutritional-Value of Termitomyces Mycelial Protein and Growth of Mycelium on Natural Substrates." Mycological Research 96: 350-354.

Bourke, P. M., G. van Geest, R. E. Voorrips, J. Jansen, T. Kranenburg, et al. (2018). "polymapR-linkage analysis and genetic map construction from F1 populations of outcrossing polyploids." Bioinformatics 34(20): 3496-3502.

Bright, M. and S. Bulgheresi (2010). "A complex journey: transmission of microbial symbionts.” Nature reviews. Microbiology 8(3): 218-230. 
Bucek, A., J. Šobotník, S. He, M. Shi, D. P. McMahon, et al. (2019). "Evolution of Termite Symbiosis Informed by Transcriptome-Based Phylogenies." Current Biology 29(21): 37283734.e3724.

Budar, F., P. Touzet and R. De Paepe (2003). "The nucleo-mitochondrial conflict in cytoplasmic male sterilities revisited." Genetica 117(1): 3-16.

Bull, J. J., I. J. Molineux and W. R. Rice (1991). "Selection of Benevolence in a Host-Parasite System." Evolution 45(4): 875-882.

Bull, J. J. and W. R. Rice (1991). "Distinguishing mechanisms for the evolution of co-operation.” Journal of Theoretical Biology 149(1): 63-74.

Buller, A. H. R. (1930). "The biological significance of conjugate nuclei in Coprinus lagopus and other hymenomycetes." Nature 126: 686-689.

Buss, L. W. (1987). The evolution of individuality. Princeton, New Jersey, Princeton University Press.

Caddick, M. X. (2004). Nitrogen Regulation in Mycelial Fungi. Biochemistry and Molecular Biology. R. Brambl and G. A. Marzluf. Berlin, Heidelberg, Springer Berlin Heidelberg: 349-368.

Callac, P., C. Billette, M. Imbernon and R. W. Kerrigan (1993). "Morphological, Genetic, and Interfertility Analyses Reveal a Novel, Tetrasporic Variety of Agaricus-Bisporus from the Sonoran Desert of California." Mycologia 85(5): 835-851.

Callac, P., C. Desmerger, R. W. Kerrigan and M. Imbernon (1997). "Conservation of genetic linkage with map expansion in distantly related crosses of Agaricus bisporus." FEMS Microbiology Letters 146(2): 235-240.

Carvalho, D. B., M. L. Smith and J. B. Anderson (1995). "Genetic Exchange between Diploid and Haploid Mycelia of Armillaria-Gallica." Mycological Research 99: 641-647.

Clark, T. A. and J. B. Anderson (2004). "Dikaryons of the basidiomycete fungus Schizophyllum commune: Evolution in long-term culture." Genetics 167(4): 1663-1675.

Coelho, M. A., G. Bakkeren, S. Sun, M. E. Hood and T. Giraud (2017). "Fungal Sex: The Basidiomycota." Microbiology spectrum 5(3).

Coolen, N. (2018). The preference of nitrogen source for different Termitomyces isolates Unpublished Internship report, Laboratory of Genetics, WUR.

Cosmides, L. M. and J. Tooby (1981). "Cytoplasmic inheritance and intragenomic conflict." J Theor Biol 89(1): 83-129.

Cotton, V. E., E. R. Hoffmann, M. F. F. Abdullah and R. H. Borts (2009). Interaction of Genetic and Environmental Factors in Saccharomyces cerevisiae Meiosis: The Devil is in the Details. Meiosis: Volume 1, Molecular and Genetic Methods. S. Keeney. Totowa, NJ, Humana Press: 3-20.

Crow, J. F. (1988). “The ultraselfish gene.” Genetics 118(3): 389-391.

Crowe, L. K. (1963). "Competition between compatible nuclei in the establishment of a dikaryon in Schizophyllum commune." Heredity 18: 525-533.

da Costa, R. R., H. Hu, B. Pilgaard, S. M. E. Vreeburg, J. Schückel, et al. (2018). "Enzyme Activities at Different Stages of Plant Biomass Decomposition in Three Species of Fungus-Growing Termites.” Applied and environmental microbiology 84(5): e01815-01817.

da Costa, R. R., S. M. E. Vreeburg, J. Z. Shik, D. K. Aanen and M. Poulsen (2019). "Can interaction specificity in the fungus-farming termite symbiosis be explained by nutritional requirements of the fungal crop?" Fungal Ecology 38: 54-61.

Dangerfield, J. M., T. S. McCarthy and W. N. Ellery (1998). "The Mound-Building Termite Macrotermes michaelseni as an Ecosystem Engineer." Journal of Tropical Ecology 14(4): 507-520.

Dangerfield, J. M. and G. Schuurman (2000). "Foraging by fungus-growing termites (Isoptera: Termitidae, Macrotermitinae) in the Okavango Delta, Botswana." Journal of Tropical Ecology 16(5): 717-731.

Darlington, J. P. E. C. (1994). Nutrition and Evolution in Fungus-growing Termites. Nourishment and Evolution in Insect Societies. J. H. Hunt and C. A. Nalepa. Boulder, Westview Press: 105-130. 
Davis, R. (1959). “Asexual Selection in Neurospora Crassa.” Genetics 44(6): 1291-1308.

De, A. B. (1983). "Basidiocarp Production by Termitomyces-Microcarpus (Berk and Br) Heim in Culture." Current Science 52(10): 494-495.

De Fine Licht, H. H., A. Andersen and D. K. Aanen (2005). "Termitomyces sp associated with the termite Macrotermes natalensis has a heterothallic mating system and multinucleate cells." Mycological Research 109: 314-318.

De Fine Licht, H. H., J. J. Boomsma and D. K. Aanen (2006). "Presumptive horizontal symbiont transmission in the fungus-growing termite Macrotermes natalensis." Molecular Ecology 15(11): 3131-3138.

De Fine Licht, H. H. D., J. J. Boomsma and D. K. Aanen (2007). "Asymmetric interaction specificity between two sympatric termites and their fungal symbionts." Ecological Entomology 32(1): 76-81.

de Vries, R. P., R. Riley, A. Wiebenga, G. Aguilar-Osorio, S. Amillis, et al. (2017). "Comparative genomics reveals high biological diversity and specific adaptations in the industrially and medically important fungal genus Aspergillus." Genome Biology 18(1): 28.

Dick, M. D. (1987). "Sexual reproduction: nuclear cycles and life-histories with particular reference to lower eukaryotes.” Biological Journal of the Linnean Society 30: 181-192.

Dimalanta, E. T., A. Lim, R. Runnheim, C. Lamers, C. Churas, et al. (2004). "A Microfluidic System for Large DNA Molecule Arrays.” Analytical Chemistry 76(18): 5293-5301.

Dixon, P. A. (1983). "Reproductive capacity of Termitomyces striatus." Transactions of the British Mycological Society 80(1): 131-139.

Dons, J. J. M., O. M. H. De Vries and J. G. H. Wessels (1979). "Characterization of the genome of the basidiomycete Schizophyllum commune.” Biochimica et Biophysica Acta (BBA) Nucleic Acids and Protein Synthesis 563(1): 100-112.

Douglas, A. E. (2010). The symbiotic habit. Princeton, N.J., Princeton University Press.

Dussutour, A., T. Latty, M. Beekman and S. J. Simpson (2010). "Amoeboid organism solves complex nutritional challenges." Proceedings of the National Academy of Sciences 107(10): 4607-4611.

Eggleton, P. (2000). Global Patterns of Termite Diversity. Termites: Evolution, Sociality, Symbioses, Ecology. T. Abe, D. E. Bignell and M. Higashi. Dordrecht, Springer Netherlands: 25-51.

Ellers, J., E. Toby Kiers, C. R. Currie, B. R. McDonald and B. Visser (2012). "Ecological interactions drive evolutionary loss of traits.” Ecology Letters 15(10): 1071-1082.

Ellingboe, A. H. and J. R. Raper (1962). "Somatic recombination in Schizophyllum commune." Genetics 47: 85-98.

Ellingboe, A. H. (1964). "Nuclear Migration in Dikaryotic-Homokaryotic Matings in Schizophyllum Commune." American Journal of Botany 51(2): 133-\&.

Elshire, R. J., J. C. Glaubitz, Q. Sun, J. A. Poland, K. Kawamoto, et al. (2011). "A robust, simple genotyping-by-sequencing (GBS) approach for high diversity species.” PLoS One 6(5): e19379.

Evans, H. J. (1959). "Nuclear Behaviour in the Cultivated Mushroom.” Chromosoma 10(2): $115-135$.

Faino, L., M. F. Seidl, E. Datema, G. C. M. van den Berg, A. Janssen, et al. (2015). "Single-Molecule Real-Time Sequencing Combined with Optical Mapping Yields Completely Finished Fungal Genome." mBio 6(4): e00936-00915.

Feeney, M. J., A. M. Miller and P. Roupas (2014). "Mushrooms-Biologically Distinct and Nutritionally Unique: Exploring a "Third Food Kingdom".” Nutrition today 49(6): 301-307.

Foulongne-Oriol, M., C. Spataro, V. Cathalot, S. Monllor and J.-M. Savoie (2010). "An expanded genetic linkage map of an intervarietal Agaricus bisporus var. bisporus $\times$ A. bisporus var. burnettii hybrid based on AFLP, SSR and CAPS markers sheds light on the recombination behaviour of the species." Fungal Genetics and Biology 47(3): 226-236.

Foulongne-Oriol, M., R. Dufourcq, C. Spataro, C. Devesse, A. Broly, et al. (2011). "Comparative linkage mapping in the white button mushroom Agaricus bisporus provides foundation for breeding management." Current Genetics 57(1): 39-50. 
Frank, S. A. (1989). “The Evolutionary Dynamics of Cytoplasmic Male Sterility.” American Naturalist 133(3): 345-376.

Frank, S. A. (1996). "Host-symbiont conflict over the mixing of symbiotic lineages." Proceedings of the Royal Society of London. Series B: Biological Sciences 263(1368): 339.

Freihorst, D., T. J. Fowler, K. Bartholomew, M. Raudaskoski, J. S. Horton, et al. (2016). The Mating-Type Genes of Basidiomycetes. The Mycota I, Growth, Differentiation and Sexuality, 3rd edition. J. Wendland, Springer International Publishing Switzerland: 329-349.

Freitag, M., P. C. Hickey, N. B. Raju, E. U. Selker and N. D. Read (2004). "GFP as a tool to analyze the organization, dynamics and function of nuclei and microtubules in Neurospora crassa." Fungal Genetics and Biology 41(10): 897-910.

Fritsche, G. (1986). "Breeding Mushrooms." Mushroom J. 157: 4-17.

FrØSlev, T. G., D. K. Aanen, T. LÆSs ØE and S. Rosendahl (2003). "Phylogenetic relationships of Termitomyces and related taxa." Mycological Research 107(11): 1277-1286.

Gaertner, J. (2019). An analysis of substrate breakdown in Termitomyces sp. grown on various complex substrates Unpublished BSc thesis, Laboratory of Genetics, WUR.

Garnier-Sillam, E., F. Toutain, G. Villemin and J. Renoux (1989). "Études Préliminaires des Meules Originales du Termite Xylophage Sphaerotermes sphaerothorax (Sjostedt).” Insectes Sociaux 36(4): 293-312.

Garrison, E. and G. Marth (2012). "Haplotype-based variant detection from short-read sequencing." arXiv:1207.3907.

Geisseler, D., W. R. Horwath, R. G. Joergensen and B. Ludwig (2010). "Pathways of nitrogen utilization by soil microorganisms - A review." Soil Biology and Biochemistry 42(12): 2058-2067.

Gerber, C., S. Badertscher and R. H. Leuthold (1988). "Polyethism in Macrotermes bellicosus (Isoptera)." Insectes Sociaux 35(3): 226-240.

Gladfelter, A. and J. Berman (2009). "Dancing genomes: fungal nuclear positioning." Nat Rev Microbiol 7(12): 875-886.

Gondwana Collection Namibia. (2017). from https://www.gondwana-collection.com/news/ article/2017/03/03/omajova-a-wild-delicacy/.

Gong, W.-B., W. Liu, Y.-Y. Lu, Y.-B. Bian, Y. Zhou, et al. (2014). "Constructing a new integrated genetic linkage map and mapping quantitative trait loci for vegetative mycelium growth rate in Lentinula edodes." Fungal Biology 118(3): 295-308.

Grant, P. R. (1991). "Natural Selection and Darwin's Finches.” Scientific American 265(4): 8287.

Grant, R. B. and P. R. Grant (2003). "What Darwin's Finches Can Teach Us about the Evolutionary Origin and Regulation of Biodiversity." BioScience 53(10): 965-975.

Grassé, P. P. and C. Noirot (1958). "Le meule des termites champignonnistes et sa signification symbiotique.” Ann Sci. Nat: Zool. Biol. Anim. 20: 113-128.

Grassé, P. P. (1982). Termitologia. Paris, Masson.

Groenendijk, J. (2016, April 20, 2016). from https://www.jessicagroenendijk.com/single-post/2016/04/19/Titan-of-the-Forest-1.

Gurevich, A., V. Saveliev, N. Vyahhi and G. Tesler (2013). "QUAST: quality assessment tool for genome assemblies.” Bioinformatics 29(8): 1072-1075.

Haig, D. and A. Wilczek (2006). "Sexual conflict and the alternation of haploid and diploid generations." Philosophical Transactions of the Royal Society B-Biological Sciences 361(1466): 335-343.

Haig, D. (2016). "Living together and living apart: the sexual lives of bryophytes." Philosophical Transactions of the Royal Society B: Biological Sciences 371(1706): 20150535.

Haitham, A. H. (2010). Investigation of flow through and around the Macrotermes michaelseni termite mound skin. Doctoral Thesis, Loughborough University.

Halbwachs, H., J. Simmel and C. Bässler (2016). "Tales and mysteries of fungal fruiting: How morphological and physiological traits affect a pileate lifestyle." Fungal Biology Reviews 30(2): 36-61.

Hamilton, W. D. and R. M. May (1977). "Dispersal in stable habitats." Nature 269: 578. 
Hao, Y.-J., Q. Zhao and S. Wang (2016). "What is the radicate Oudemansiella cultivated in China?" Phytotaxa 286: 1.

Heath, K. D. and J. R. Stinchcombe (2014). "Explaining mutualism variation: a new evolutionary paradox?" Evolution 68(2): 309-317.

Heaton, L. L. M., E. López, P. K. Maini, M. D. Fricker and N. S. Jones (2010). "Growth-induced mass flows in fungal networks." Proceedings of the Royal Society B: Biological Sciences 277(1698): 3265-3274.

Heim, R. (1942). Les champignons des termitières. Nouveaux aspects d'un problèm de biologie et de systématique générale, La Revue Scientifique.

Heim, R. (1977). Termites et champignons: les champignons termitophiles d'Afrique noire et d'Asie méridionale, Bouhée.

Heinzelmann, R., D. Rigling, G. Sipos, M. Münsterkötter and D. Croll (2020). "Chromosomal assembly and analyses of genome-wide recombination rates in the forest pathogenic fungus Armillaria ostoyae.” Heredity 124(6): 699-713.

Hensen, L. (2018). The effect of different nitrogen sources on the growth of 23 Termitomyces strains Unpublished BSc thesis, Laboratory of Genetics, WUR.

Herre, E. A., N. Knowlton, U. G. Mueller and S. A. Rehner (1999). "The evolution of mutualisms: exploring the paths between conflict and cooperation." Trends in Ecology \& Evolution 14(2): 49-53.

Hinze, B. and R. H. Leuthold (1999). "Age related polyethism and activity rhythms in the nest of the termite Macrotermes bellicosus (Isoptera, Termitidae).” Insectes Sociaux 46(4): 392-397.

Hinze, B., K. Crailsheim and R. H. Leuthold (2002). "Polyethism in food processing and social organisation in the nest of Macrotermes bellicosus (Isoptera, Termitidae)." Insectes Sociaux 49(1): 31-37.

Hoekstra, R. F. (1990). Evolution of uniparental inheritance of cytoplasmic DNA. Organisational constraints on the dynamics of evolution. J. Maynard Smith and G. Vida. Manchester, New York, Manchester Univ. Press: 269-278.

Hui, C., H. Yamamoto, K. Takeo and Y. Kitamoto (1999). "Nuclear selection in monokaryotic oidium formation from dikaryotic mycelia in a basidiomycete, Pholiota nameko." Mycoscience 40: 199-203.

Hurst, L. D., A. Atlan and B. O. Bengtsson (1996). "Genetic conflicts." Quarterly Review of Biology 71(3): 317-364.

Hyodo, F., T. Inoue, J. I. Azuma, I. Tayasu and T. Abe (2000). "Role of the mutualistic fungus in lignin degradation in the fungus-growing termite Macrotermes gilvus (Isoptera; Macrotermitinae)." Soil Biology and Biochemistry 32(5): 653-658.

Iwasa, M., S. Tanabe and T. Kamada (1998). "The Two Nuclei in the Dikaryon of the Homobasidiomycete Coprinus cinereus Change Position after Each Conjugate Division." Fungal Genet Biol 23(1): 110-116.

James, T. Y. and R. Vilgalys (2001). "Abundance and diversity of Schizophyllum commune spore clouds in the Caribbean detected by selective sampling." Mol Ecol 10(2): 471-479.

James, T. Y., U. Kues, S. A. Rehner and R. Vilgalys (2004). "Evolution of the gene encoding mitochondrial intermediate peptidase and its cosegregation with the A mating-type locus of mushroom fungi." Fungal Genet Biol 41(3): 381-390.

James, T. Y., P. Srivilai, U. Kües and R. Vilgalys (2006). "Evolution of the bipolar mating system of the mushroom Coprinellus disseminatus from its tetrapolar ancestors involves loss of mating-type-specific pheromone receptor function." Genetics 172(3): 1877-1891.

James, T. Y., J. Stenlid, A. Olson and H. Johannesson (2008). "Evolutionary significance of imbalanced nuclear ratios within heterokaryons of the basidiomycete fungus Heterobasidion parviporum." Evolution 62(9): 2279-2296.

James, T. Y. (2015). "Why mushrooms have evolved to be so promiscuous: Insights from evolutionary and ecological patterns.” Fungal Biology Reviews 29(3-4): 167-178. 
Johjima, T., Y. Taprab, N. Noparatnaraporn, T. Kudo and M. Ohkuma (2006). "Large-scale identification of transcripts expressed in a symbiotic fungus (Termitomyces) during plant biomass degradation.” Applied Microbiology and Biotechnology 73(1): 195-203.

Johnson, R. A. (1981). "Colony development and establishment of the fungus comb in Microtermes sp. nr. Usambaricus (Sjöstedt) (Isoptera: Macrotermitinae) from Nigeria.” Insectes Sociaux 28(1): 3-12.

Johnson, R. A., R. J. Thomas, T. G. Wood and M. J. Swift (1981). "The inoculation of the fungus comb in newly founded colonies of some species of the Macrotermitinae (Isoptera) from Nigeria." Journal of Natural History 15(5): 751-756.

Josse, J. and F. Husson (2016). "missMDA: A Package for Handling Missing Values in Multivariate Data Analysis.” 2016 70(1): 31.

Jouquet, P., E. Chaudhary and A. R. V. Kumar (2017). "Sustainable use of termite activity in agro-ecosystems with reference to earthworms. A review." Agronomy for Sustainable Development 38(1): 3 .

Kansci, G., D. C. Mossebo, A. B. Selatsa and M. Fotso (2003). "Nutrient content of some mushroom species of the genus Termitomyces consumed in Cameroon." Nahrung-Food 47(3): 213-216.

Kazuhiro, M., F. Huang, B. Zhang, S. Shiraishi, M. Sakai, et al. (2008). "Genetic map of a basidiomycete fungus, Lentinula edodes (shiitake mushroom), constructed by tetrad analysis." Breeding Science 58: 23-30.

Kearse, M., R. Moir, A. Wilson, S. Stones-Havas, M. Cheung, et al. (2012). “Geneious Basic: an integrated and extendable desktop software platform for the organization and analysis of sequence data." Bioinformatics 28(12): 1647-1649.

Kerrigan, R. W., J. C. Royer, L. M. Baller, Y. Kohli, P. A. Horgen, et al. (1993). "Meiotic behavior and linkage relationships in the secondarily homothallic fungus Agaricus bisporus." Genetics 133(2): 225-236.

Kim, D., Y. Tamai, T. Azuma, A. Harada, A. Ando, et al. (2000). "Analysis of the electrophoretic karyotype of Flammulina velutipes.” Journal of Wood Science 46(6): 466-469.

Kitamoto, Y., M. Shishida, H. Yamamoto, K. Takeo and P. Masuda (2000). "Nuclear selection in oidium formation from dikaryotic mycelia of Flammulina velutipes." Mycoscience 41: 417-423.

Kohler, C. and I. Weinhofer-Molisch (2010). "Mechanisms and evolution of genomic imprinting in plants." Heredity 105(1): 57-63.

Kone, N. A., K. Dosso, S. Konate, J. Y. Kouadio and K. E. Linsenmair (2011). "Environmental and biological determinants of Termitomyces species seasonal fructification in central and southern Cote d'Ivoire." Insectes Sociaux 58(3): 371-382.

Kone, N. A., K. Yeo and K. E. Linsenmair (2013). "Socio-economical aspects of the exploitation of Termitomyces fruit bodies in central and southern Côte d'Ivoire: Raising awareness for their sustainable use." Journal of Applied Biosciences 70: 5580- 5590.

Kooij, P. W. (2013). Fungal adaptations to mutualistic life with ants Doctoral thesis, Centre for Social Evolution, University of Copenhagen.

Kooij, P. W., D. K. Aanen, M. Schiøtt and J. J. Boomsma (2015). "Evolutionarily advanced ant farmers rear polyploid fungal crops." Journal of Evolutionary Biology 28(11): 1911-1924.

Korb, J. (2003). "Thermoregulation and ventilation of termite mounds." Naturwissenschaften 90(5): 212-219.

Korb, J. and D. K. Aanen (2003). "The evolution of uniparental transmission of fungal symbionts in fungus-growing termites (Macrotermitinae)." Behavioral Ecology and Sociobiology 53(2): 65-71.

Koren, S., B. P. Walenz, K. Berlin, J. R. Miller, N. H. Bergman, et al. (2017). "Canu: scalable and accurate long-read assembly via adaptive k-mer weighting and repeat separation." Genome Res 27(5): 722-736.

Kues, U. (2000). "Life history and developmental processes in the basidiomycete Coprinus cinereus." Microbiol Mol Biol Rev 64(2): 316-353. 
Kües, U. and Y. Liu (2000). "Fruiting body production in basidiomycetes." Applied Microbiology and Biotechnology 54(2): 141-152.

Kües, U., S. M. Badalyan, A. Gießler and B. Dörnte (2016). Asexual Sporulation in Agaricomycetes. Growth, Differentiation and Sexuality. J. Wendland. Cham, Springer International Publishing: 269-328.

LaFage, J. P. and W. L. Nutting (1978). Nutrient dynamics of termites. Production Ecology of Ants and Termites. M. V. Brian, Cambridge University Press: 165-232.

Larraya, L. M., G. Pérez, E. Ritter, A. G. Pisabarro and L. Ramírez (2000). "Genetic linkage map of the edible basidiomycete Pleurotus ostreatus." Applied and environmental microbiology 66(12): 5290-5300.

Lavelle, P., D. Bignell, L. M, V. Wolters, P. Roger, et al. (1997). "Soil function in a changing world: The role of invertebrate ecosystem engineers." European Journal of Soil Science 33: 159-193.

Law, R. and D. H. Lewis (1983). "Biotic environments and the maintenance of sex-some evidence from mutualistic symbioses.” Biological Journal of the Linnean Society 20(3): 249276.

Lê, S., J. Josse and F. Husson (2008). "FactoMineR: An R Package for Multivariate Analysis." 2008 25(1): 18.

Lee, K. P., S. J. Simpson, F. J. Clissold, R. Brooks, J. W. O. Ballard, et al. (2008). "Lifespan and reproduction in Drosophila: New insights from nutritional geometry." Proceedings of the National Academy of Sciences 105(7): 2498-2503.

Lee, S. H., M. K. Kim, M. K. Lee, N. R. Kim, C.-Y. Lee, et al. (2014). "Electrophoretic karyotyping of Hypsizygus marmoreus and evaluation of variation among its basidiospores." FEMS Microbiology Letters 359(2): 209-215.

Leigh Jr, E. G. (2010). "The evolution of mutualism.” Journal of Evolutionary Biology 23(12): $2507-2528$.

Leuthold, R. H., S. Badertscher and H. Imboden (1989). "The inoculation of newly formed fungus comb with Termitomyces in Macrotermes colonies (Isoptera, Macrotermitinae)." Insectes Sociaux 36(4): 328-338.

Lewin, H. A., D. M. Larkin, J. Pontius and S. J. O’Brien (2009). “Every genome sequence needs a good map." Genome Res 19(11): 1925-1928.

Li, H., B. Handsaker, A. Wysoker, T. Fennell, J. Ruan, et al. (2009). "The Sequence Alignment/ Map format and SAMtools." Bioinformatics 25(16): 2078-2079.

Li, H. (2013). "Aligning sequence reads, clone sequences and assembly contigs with BWAMEM." arXiv:1303.3997v1.

Li, H. (2018). "Minimap2: pairwise alignment for nucleotide sequences.” Bioinformatics 34(18): 3094-3100.

Li, H. J., C. Dietrich, N. Zhu, A. Mikaelyan, B. Ma, et al. (2016). "Age polyethism drives community structure of the bacterial gut microbiota in the fungus-cultivating termite Odontotermes formosanus." Environmental Microbiology 18(5): 1440-1451.

Lloyd, A., C. Morgan, F. C. H Franklin and K. Bomblies (2018). "Plasticity of Meiotic Recombination Rates in Response to Temperature in Arabidopsis." Genetics 208(4): 1409-1420.

Malek, S. N. A., G. Kanagasabapathy, V. Sabaratnam, N. Abdullah and H. Yaacob (2012). "Lipid Components of a Malaysian Edible Mushroom, Termitomyces Heimii Natarajan." International Journal of Food Properties 15(4): 809-814.

Marais, E. (1937). The Soul of the White Ant, dminoz.

Masamba, K. (2010). "Determination and comparison of nutrient and mineral contents between cultivated and indigenous edible mushrooms in central Malawi." African Journal of food science 4: 176-179.

Matsumoto, T. and Y. Fukumasa-Nakai (1996). "Mitochondrial DNA inheritance in sexual crosses of Pleurotus ostreatus." Current Genetics : Lower Eukaryotes and Organelles 30(6): 549-552.

May, G. and J. W. Taylor (1988). "Patterns of mating and mitochondrial DNA inheritance in the agaric Basidiomycete Coprinus cinereus." Genetics 118(2): 213-220. 
May, G., F. Shaw, H. Badrane and X. Vekemans (1999). "The signature of balancing selection: fungal mating compatibility gene evolution.” Proc Natl Acad Sci U S A 96(16): 9172-9177.

Maynard Smith, J. (1989). "Generating novelty by symbiosis." Nature 341(6240): 284-285.

Maynard Smith, J. and E. r. Szathmáry (1995). The major transitions in evolution. Oxford, W.H. Freeman Spektrum.

Mitchell, J. D. (2002). "Termites as pests of crops, forestry, rangeland and structures in southern Africa and their control." Sociobiology 40: 47-69.

Moore, D. (1994). Tissue Formation. The Growing Fungus. N. A. R. Gow and G. M. Gadd. London, Chapman \& Hall: 423-465.

Moya, A., J. Peretó, R. Gil and A. Latorre (2008). "Learning how to live together: genomic insights into prokaryote-animal symbioses." Nature reviews. Genetics 9(3): 218-229.

Mueller, U. G. (2002). "Ant versus fungus versus mutualism: ant-cultivar conflict and the deconstruction of the attine ant-fungus symbiosis." Am Nat 160 Suppl 4: S67-98.

Nieuwenhuis, B. P., A. J. Debets and D. K. Aanen (2011). "Sexual selection in mushroom-forming basidiomycetes." Proc Biol Sci 278(1702): 152-157.

Nieuwenhuis, B. P. and D. K. Aanen (2012). "Sexual selection in fungi." J Evol Biol 25(12): 2397-2411.

Nieuwenhuis, B. P. S., S. Nieuwhof and D. K. Aanen (2013). "On the asymmetry of mating in natural populations of the mushroom fungus Schizophyllum commune.” Fungal Genetics and Biology 56: 25-32.

Nieuwenhuis, B. P. S. and D. K. Aanen (2018). "Nuclear arms races: Experimental evolution for mating success in the mushroom-forming fungus Schizophyllum commune." PLOS ONE 13(12): e0209671.

Nieuwenhuis, M., L. J. J. van de Peppel, F. T. Bakker, B. J. Zwaan and D. K. Aanen (2019). "Enrichment of G4DNA and a Large Inverted Repeat Coincide in the Mitochondrial Genomes of Termitomyces." Genome Biology and Evolution 11(7): 1857-1869.

Nobre, T., P. Eggleton and D. K. Aanen (2010). "Vertical transmission as the key to the colonization of Madagascar by fungus-growing termites?” Proceedings of the Royal Society B: Biological Sciences 277(1680): 359-365.

Nobre, T., C. Fernandes, J. J. Boomsma, J. Korb and D. K. Aanen (2011a). "Farming termites determine the genetic population structure of Termitomyces fungal symbionts." Molecular Ecology 20(9): 2023-2033.

Nobre, T., N. A. Kone, S. Konate, K. E. Linsenmair and D. K. Aanen (2011b). "Dating the fungus-growing termites' mutualism shows a mixture between ancient codiversification and recent symbiont dispersal across divergent hosts." Mol Ecol 20(12): 2619-2627.

Nobre, T., C. Rouland-Lefèvre and D. K. Aanen (2011c). Comparative Biology of Fungus Cultivation in Termites and Ants. Biology of Termites: a Modern Synthesis. D. E. Bignell, Y. Roisin and N. Lo. Dordrecht, Springer Netherlands: 193-210.

Nobre, T. and D. K. Aanen (2012). "Fungiculture or Termite Husbandry? The Ruminant Hypothesis." Insects(3): 307-323.

Nobre, T., B. Koopmanschap, J. J. P. Baars, A. S. M. Sonnenberg and D. K. Aanen (2014). “The scope for nuclear selection within Termitomyces fungi associated with fungus-growing termites is limited.” Bmc Evolutionary Biology 14.

Ogundana, S. K. and O. E. Fagade (1982). "Nutritive-Value of Some Nigerian Edible Mushrooms." Food Chemistry 8(4): 263-268.

Ohm, R. A., J. F. de Jong, L. G. Lugones, A. Aerts, E. Kothe, et al. (2010). "Genome sequence of the model mushroom Schizophyllum commune.” Nat Biotechnol 28(9): 957-963.

Okuda, Y., J. Ueda, Y. Obatake, S. Murakami, Y. Fukumasa, et al. (2012). "Construction of a Genetic Linkage Map Based on Amplified Fragment Length Polymorphism Markers and Development of Sequence-Tagged Site Markers for Marker-Assisted Selection of the Sporeless Trait in the Oyster Mushroom." Applied and Environmental Microbiology 78(5): 1496-1504.

Ono, K., T. Hata, T. Yoshimura and K. Kinjo (2017). "Wood decaying properties of the termite mushroom Termitomyces eurrhizus.” Journal of Wood Science 63(1): 83-94. 
Orbach, M. J., D. Vollrath, R. W. Davis and C. Yanofsky (1988). "An electrophoretic karyotype of Neurospora crassa." Molecular and Cellular Biology 8(4): 1469-1473.

Otani, S., A. Mikaelyan, T. Nobre, L. H. Hansen, N. G. A. Koné, et al. (2014). "Identifying the core microbial community in the gut of fungus-growing termites." Molecular Ecology 23(18): 4631-4644.

Ouellette, L. A., R. W. Reid, S. G. Blanchard and C. R. Brouwer (2018). "LinkageMapView-rendering high-resolution linkage and QTL maps.” Bioinformatics 34(2): 306-307.

Packbier, N. (2018). "Genetic Transformation of Termitomyces associated with Macrotermes natalensis, MSc thesis."

Park, S.-K., M. M. Peñas, L. Ramírez and A. G. Pisabarro (2006). "Genetic linkage map and expression analysis of genes expressed in the lamellae of the edible basidiomycete Pleurotus ostreatus." Fungal Genetics and Biology 43(5): 376-387.

Pegler, D. N. and G. D. Piearce (1980). “The Edible Mushrooms of Zambia.” Kew Bulletin 35(3): 475-491.

Pegler, D. N. and M. Vanhaecke (1994). “Termitomyces of Southeast Asia.” Kew Bulletin 49(4): 717-736.

Petch, T. (1906). "The Fungi of certain Termite Nests " Annals of the Royal Botanic Gardens, Peradeniya 3: $185-270$.

Piearce, G. D. (1987). “The genus Termitomyces in Zambia.” Mycologist 1(3): 111-116.

Potrikus, C. J. and J. A. Breznak (1980). "Uric Acid-Degrading Bacteria in Guts of Termites Reticulitermes flavipes (Kollar).” Applied and Environmental Microbiology 40(1): 117 124.

Potrikus, C. J. and J. A. Breznak (1981). "Gut bacteria recycle uric acid nitrogen in termites: A strategy for nutrient conservation." Proceedings of the National Academy of Sciences 78(7): 4601-4605.

Poulsen, M. and J. Boomsma (2005). "Mutualistic Fungi Control Crop Diversity in Fungus-Growing Ants.” Science (New York, N.Y.) 307: 741-744.

Poulsen, M., H. F. Hu, C. Li, Z. S. Chen, L. H. Xu, et al. (2014). "Complementary symbiont contributions to plant decomposition in a fungus-farming termite." Proceedings of the National Academy of Sciences of the United States of America 111(40): 14500-14505.

Preedy, K. F. and C. A. Hackett (2016). "A rapid marker ordering approach for high-density genetic linkage maps in experimental autotetraploid populations using multidimensional scaling." Theor Appl Genet 129(11): 2117-2132.

Queller, D. C. (1994). "Extended parental care and the origin of eusociality." Proceedings of the Royal Society of London. Series B: Biological Sciences 256(1346): 105-111.

Queller, D. C. and J. E. Strassmann (2009). "Beyond society: the evolution of organismality." Philosophical Transactions of the Royal Society B-Biological Sciences 364(1533): 31433155.

Quintanilha, A. (1937). “Contribution à l'étude génétique du phénomène de Buller.” C.R. Soc. Biol. (Paris) 205: 745.

R Core Team (2019). R: A Language and Environment for Statistical Computing, R Foundation for Statistical Computing.

Ramsdale, M. and A. D. M. Rayner (1994). "Distribution patterns of number of nuclei in conidia from heterokaryons of Heterobasidion annosum (Fr) Bref. and their interpretation in terms of genomic conflict." New Phytologist 128: 123-134.

Ramsdale, M. and A. D. M. Rayner (1996). "Imbalanced nuclear ratios, postgermination mortality and phenotype-genotype relationships in allopatrically-derived heterokaryons of Heterobasidion annosum." New Phytologist 133: 303-319.

Ramsdale, M. (1999). "Genomic conflict in fungal mycelia." Population and community biology series 25(139-174).

Raper, C. A., J. R. Raper and R. E. Miller (1972). "Genetic analysis of the life cycle of Agaricus bisporus." Mycologia 64: 1088-1117.

Raper, J. R. (1966). Genetics of sexuality in higher fungi. New York, Ronald Press. 
Rastas, P. (2017). "Lep-MAP3: robust linkage mapping even for low-coverage whole genome sequencing data." Bioinformatics 33(23): 3726-3732.

Raubenheimer, D. (2011). "Toward a quantitative nutritional ecology: the right-angled mixture triangle.” Ecological Monographs 81(3): 407-427.

Rech, C., I. Engh and U. Kück (2007). "Detection of hyphal fusion in filamentous fungi using differently fluorescence-labeled histones." Current Genetics 52(5): 259.

Reik, W. and J. Walter (2001). "Genomic imprinting: Parental influence on the genome." Nature Reviews Genetics 2(1): 21-32.

Rizzo, D. M. and G. May (1994). "Nuclear Replacement during Mating in Armillaria-Ostoyae (Basidiomycotina)." Microbiology-Uk 140: 2115-2124.

Roberts, E. M., C. N. Todd, D. K. Aanen, T. Nobre, H. L. Hilbert-Wolf, et al. (2016). “Oligocene Termite Nests with In Situ Fungus Gardens from the Rukwa Rift Basin, Tanzania, Support a Paleogene African Origin for Insect Agriculture.” PLoS One 11(6): e0156847.

Robinson, J. T., H. Thorvaldsdottir, W. Winckler, M. Guttman, E. S. Lander, et al. (2011). "Integrative genomics viewer." Nat Biotechnol 29(1): 24-26.

Rouland-Lefèvre, C. (2000). Symbiosis With Fungi. Termites: Evolution, Sociality, Symbioses, Ecology. T. Abe, D. E. Bignell and M. Higashi. Dordrecht, Springer Netherlands: 289-306.

Rouland-Lefèvre, C. and D. E. Bignell (2002). Cultivation of Symbiotic Fungi by Termites of the Subfamily Macrotermitinae. Symbiosis: Mechanisms and Model Systems. J. Seckbach. Dordrecht, Springer Netherlands: 731-756.

Rouland-Lefèvre, C. (2011). Termites as Pests of Agriculture. Biology of Termites: a Modern Synthesis. D. E. Bignell, Y. Roisin and N. Lo. Dordrecht, Springer Netherlands: 499-517.

Runderkamp, M. (2016). Nutrient requirements for Termitomyces species Unpublished BSc Thesis.

Sachs, J. L., U. G. Mueller, T. P. Wilcox and J. J. Bull (2004). “The evolution of cooperation.” Q Rev Biol 79(2): 135-160.

Sakamoto, Y. (2018). "Influences of environmental factors on fruiting body induction, development and maturation in mushroom-forming fungi.” Fungal Biology Reviews 32(4): 236248.

Saksena, K. N., R. Marino, M. N. Haller and P. A. Lemke (1976). "Study on Development of Agaricus-Bisporus by Fluorescent Microscopy and Scanning Electron-Microscopy.” Journal of Bacteriology 126(1): 417-428.

Samils, N., J. Oliva and H. Johannesson (2014). "Nuclear interactions in a heterokaryon: insight from the model Neurospora tetrasperma." Proceedings of the Royal Society B-Biological Sciences 281(1786).

Sands, W. A. (1960). "The initiation of fungus comb construction in laboratory colonies of Ancistrotermes guineensis (Silvestri).” Insectes Sociaux 7(3): 251-263.

Sedaghat Telgerd, N. (2017). Unravelling the genetic base of the meiotic recombination landscapes in two varieties of the button mushroom, Agaricus bisporus Doctoral thesis, Wageningen University.

Seidl, M. F., L. Faino, X. Shi-Kunne, G. C. M. van den Berg, M. D. Bolton, et al. (2015). "The Genome of the Saprophytic Fungus Verticillium tricorpus Reveals a Complex Effector Repertoire Resembling That of Its Pathogenic Relatives.” Molecular Plant-Microbe Interactions ${ }^{\circledR} 28(3): 362-373$.

Shik, J. Z., E. B. Gomez, P. W. Kooij, J. C. Santos, W. T. Wcislo, et al. (2016). "Nutrition mediates the expression of cultivar-farmer conflict in a fungus-growing ant." Proceedings of the National Academy of Sciences 113(36): 10121-10126.

Sieber, R. (1983). "Establishment of fungus comb in Laboratory colonies of Macrotermes michaelseni and Odontotermes montanus (Isoptera, Macrotermitinae)." Insectes Sociaux 30(2): 204-209.

Simao, F. A., R. M. Waterhouse, P. Ioannidis, E. V. Kriventseva and E. M. Zdobnov (2015). "BUSCO: assessing genome assembly and annotation completeness with single-copy orthologs." Bioinformatics 31(19): 3210-3212. 
Simpson, S. J. and D. Raubenheimer (2012). The Nature of Nutrition; A Unifying Framework from Animal Adaptation to Human Obesity, Princeton University Press.

Slaytor, M. and D. J. Chappell (1994). "Nitrogen metabolism in termites." Comparative Biochemistry and Physiology -- Part B: Biochemistry and Molecular Biology 107(1): 1-10.

Slot, J. C. and D. S. Hibbett (2007). "Horizontal Transfer of a Nitrate Assimilation Gene Cluster and Ecological Transitions in Fungi: A Phylogenetic Study." PLOS ONE 2(10): e1097.

Smith, K. M., J. M. Galazka, P. A. Phatale, L. R. Connolly and M. Freitag (2012). "Centromeres of filamentous fungi." Chromosome research : an international journal on the molecular, supramolecular and evolutionary aspects of chromosome biology 20(5): 635-656.

Snider, P. J. and J. R. Raper (1958). "Nuclear migration in the basidiomycete Schizophyllum commune." American Journal of Botany 45(7): 538-546.

Soleymaninejadian, E., B. Z. Ji, S. W. Liu, J. J. Yang and X. W. Zhang (2014). "Foraging Polyethism in Odontotermes Formosanus Shiraki." Journal of Advanced Agricultural Technologies I(1): 32-42.

Sonnenberg, A. S., P. W. de Groot, P. J. Schaap, J. J. Baars, J. Visser, et al. (1996). "Isolation of expressed sequence tags of Agaricus bisporus and their assignment to chromosomes." Applied and environmental microbiology 62(12): 4542-4547.

Sonnenberg, A. S. M., J. J. P. Baars, P. M. Hendrickx, B. Lavrijssen, W. Gao, et al. (2011). Breeding and strain protection in the button mushroom Agaricus bisporus. proceedings of the 7th International Conference of the World Society for Mushroom Biology and Mushroom Products, 04-07 October 2011, Arcachon, France, ICMBMP.

Sonnenberg, A. S. M., W. Gao, B. Lavrijssen, P. Hendrickx, N. Sedaghat-Tellgerd, et al. (2016). "A detailed analysis of the recombination landscape of the button mushroom Agaricus bisporus var. bisporus." Fungal Genetics and Biology 93: 35-45.

Stapley, J., P. G. D. Feulner, S. E. Johnston, A. W. Santure and C. M. Smadja (2017). "Variation in recombination frequency and distribution across eukaryotes: patterns and processes.” Philosophical Transactions of the Royal Society B: Biological Sciences 372(1736): 20160455.

Stearns, S. C. and R. F. Hoekstra (2000). Evolution: An Introduction, Oxford University Press.

Swietzynski, K. M. and P. R. Day (1960). "Heterokaryon formation in Coprinus lagopus." Genet. Res. Camb. 1: 114-128.

Tayasu, I., F. Hyodo and T. Abe (2002). "Caste-specific N and C isotope ratios in fungus-growing termites with special reference to uric acid preservation and their nutritional interpretation.” Ecological Entomology 27(3): 355-361.

Teague, B., M. S. Waterman, S. Goldstein, K. Potamousis, S. Zhou, et al. (2010). "High-resolution human genome structure by single-molecule analysis." Proceedings of the National Academy of Sciences 107(24): 10848-10853.

Terashima, K., T. Matsumoto, E. Hayashi and Y. Fukumasa-Nakai (2002). "A genetic linkage map of Lentinula edodes (shiitake) based on AFLP markers." Mycological Research 106(8): 911-917.

Thomas, R. J. (1987a). "Distribution of Termitomyces heim and other fungi in the nests and major workers of Macrotermes bellicosus (Smeathman) in Nigeria." Soil Biology and Biochemistry 19(3): 329-333.

Thomas, R. J. (1987b). "Factors affecting the distribution and activity of fungi in the nests of macrotermitinae (isoptera)." Soil Biology and Biochemistry 19(3): 343-349.

Thomma, B., M. F. Seidl, X. Shi-Kunne, D. E. Cook, M. D. Bolton, et al. (2016). "Mind the gap; seven reasons to close fragmented genome assemblies." Fungal Genet Biol 90: 24-30.

Turner, B. C. and D. D. Perkins (1991). "Meiotic drive in Neurospora and other fungi." The American Naturalist 137(3): 416-429.

Turner, J. S. (2011). “Termites as models of swarm cognition.” Swarm Intelligence 5(1): 19-43.

Ullrich, R. C. and J. B. Anderson (1978). "Sex and diploidy in Armillaria mellea." Experimental Mycology 2(2): 119-129.

Uys, V. M. (2002). A guide to the termite genera of Southern Africa. Pretoria, Plant Protection Research Institute, Agricultural Research Council. 
van de Peppel, L. J. J. and D. K. Aanen (2020). "High diversity and low host-specificity of Termitomyces symbionts cultivated by Microtermes spp. indicate frequent symbiont exchange." Fungal Ecology 45: 100917.

van de Peppel, L. J. J., M. Nieuwenhuis, B. Auxier, T. J. Baroni, T. W. Kuyper, et al. (submitted). Phylogenomics reveals ancestral predisposition of the termite-cultivated fungus Termitomyces towards a domesticated lifestyle.

van der Westhuizen, G. C. A. and A. Eicker (1990). "Species of Termitomyces occurring in South Africa." Mycological Research 94(7): 923-937.

van Kan, J. A. L., J. H. M. Stassen, A. Mosbach, T. A. J. Van Der Lee, L. Faino, et al. (2017). "A gapless genome sequence of the fungus Botrytis cinerea." Molecular Plant Pathology 18(1): 75-89.

van Oosten, L. (2016). Pulsed Field Gel Electrophoresis of Termitomyces sp. to estimate chromosome number Unpublished BSc Thesis, Laboratory of Genetics, WUR.

Vellinga, E. C., R. P. de Kok and T. D. Bruns (2003). "Phylogeny and taxonomy of Macrolepiota (Agaricaceae)." Mycologia 95(3): 442-456.

Viana-Junior, A. B., M. O. r. Côrtes, T. G. Cornelissen and F. d. S. Neves (2018). "Interactions between wood-inhabiting fungi and termites: a meta-analytical review." Arthropod-Plant Interactions : An international journal devoted to studies on interactions of insects, mites, and other arthropods with plants 12(2): 229-235.

Visser, A. A., V. I. D. Ros, Z. W. De Beer, A. J. M. Debets, E. Hartog, et al. (2009). "Levels of specificity of Xylaria species associated with fungus-growing termites: a phylogenetic approach.” Molecular Ecology 18(3): 553-567.

Visser, A. A., P. W. Kooij, A. J. M. Debets, T. W. Kuyper and D. K. Aanen (2011). "Pseudoxylaria as stowaway of the fungus-growing termite nest: Interaction asymmetry between Pseudoxylaria, Termitomyces and free-living relatives." Fungal Ecology 4(5): 322-332.

Visser, B., C. Le Lann, F. J. den Blanken, J. A. Harvey, J. J. M. van Alphen, et al. (2010). "Loss of lipid synthesis as an evolutionary consequence of a parasitic lifestyle." Proceedings of the National Academy of Sciences 107(19): 8677-8682.

Voedingscentrum. (2020). from https://www.voedingscentrum.nl/encyclopedie/vleesvervangers.aspx.

Vreeburg, S. M. E., K. Nygren and D. K. Aanen (2016). "Unholy marriages and eternal triangles: how competition in the mushroom life cycle can lead to genomic conflict." Philosophical Transactions of the Royal Society B: Biological Sciences 371(1706): 20150533.

Vreeburg, S. M. E., N.C.A. de Ruijter, B.J. Zwaan, R.R da Costa, M. Poulsen and D. K. Aanen (2020). "Asexual and sexual reproduction are two separate developmental pathways in a Termitomyces species." Biology Letters.

Walbot, V. and D. A. Petrov (2001). "Gene galaxies in the maize genome." Proceedings of the National Academy of Sciences 98(15): 8163-8164.

Walker, B. J., T. Abeel, T. Shea, M. Priest, A. Abouelliel, et al. (2014). "Pilon: an integrated tool for comprehensive microbial variant detection and genome assembly improvement." PLoS One 9(11): e112963.

Wang, H. H. and J. Y. Wu (1974). "Nuclear distribution in hyphal system of Agaricus bisporus." Mushroom Sciences 9: 23-29.

Wei, T.-Z., Y.-J. Yao, B. Wang and D. N Pegler (2005). Termitomyces bulborhizus sp. nov. from China, with a key to allied species.

Wheeler, W. M. (1911). “The ant-colony as an organism.” Journal of Morphology 22(2): 307325.

White, T. Bruns, S. Lee and J. Taylor (1990). White, T. J., T. D. Bruns, S. B. Lee, and J. W. Taylor. Amplification and direct sequencing of fungal ribosomal RNA Genes for phylogenetics: 315-322.

Willett, W., J. Rockström, B. Loken, M. Springmann, T. Lang, et al. (2019). "Food in the Anthropocene: the EAT- Lancet Commission on healthy diets from sustainable food systems." The Lancet 393(10170): 447-492. 
Wilson, E. O. (1971). The insect societies, Cambridge, Massachusetts, USA, Harvard University Press [Distributed by Oxford University Press.

Wong, K. H., M. J. Hynes and M. A. Davis (2008). "Recent Advances in Nitrogen Regulation: a Comparison between Saccharomyces cerevisiae and Filamentous Fungi." Eukaryotic Cell 7(6): 917-925.

Wood, T. G. and R. A. Johnson (1978). Abundance and vertical distribution in soil of Microtermes (Isoptera, Termitidae) in savanna woodland and agricultural ecosystems at Mokwa, Nigeria. Wroclaw [etc.], Zaklad Narodowy im. Ossolinskich - Wydawnictwo Polskiej Akademii Nauk.

Wood, T. G. and W. A. Sands (1978). The role of termites in ecosystems. Production Ecology of Ants and Termites. B. M.V. Cambridge, Cambridge Univ. Press: 245-292.

Wood, T. G. and R. J. Thomas (1989). The mutualistic association between Macrotermitinae and Termitomyces. London, Academic Press: 69-92.

Xiang, X. and R. Fischer (2004). "Nuclear migration and positioning in filamentous fungi." Fungal Genet Biol 41(4): 411-419.

Xu, J., R. W. Kerrigan, P. A. Horgen and J. B. Anderson (1993). "Localization of the Mating Type Gene in Agaricus bisporus.” Applied and environmental microbiology 59(9): 3044 3049.

Yang, R.-H., Y. Li, Y. Wáng, J.-N. Wan, C.-L. Zhou, et al. (2016). “The genome of Pleurotus eryngii provides insights into the mechanisms of wood decay." Journal of Biotechnology 239: 65-67.

Yip, P. Y., K. S. Wong, L. Li, Q. L. Huang, H. S. Kwan, et al. (2012). “Genome sequence and genetic linkage analysis of Shiitake mushroom Lentinula edodes." Nature Precedings.

Zhan, H. and S. Xu (2011). "Generalized linear mixed model for segregation distortion analysis.” BMC Genetics 12(1): 97.

Zhang, K., X.-C. Wu, D.-Q. Zheng and T. D. Petes (2017). "Effects of Temperature on the Meiotic Recombination Landscape of the Yeast Saccharomyces cerevisiae." mBio 8(6): e02099-02017.

Zhou, S., J. Herschleb and D. C. Schwartz (2007). A Single Molecule System for Whole Genome Analysis. Perspectives in Bioanalysis. K. R. Mitchelson, Elsevier. 2: 265-300. 


\section{Summary}

Life is organised in a hierarchical fashion; smaller replicating entities cooperate to make more complex organisational forms. For example, DNA is organised in genes, genes are organised on chromosomes, chromosomes are organised in nuclei, nuclei and organelles are organised in cells and cells can be organised in multicellular organisms. One form of higher-level organisation is an obligate symbiotic mutualism; two different species that become mutually dependent on each other. From an evolutionary perspective there is a tension between lower-level selection and higher-level organisation; natural selection at the lower level can oppose the higher-level organisation, if reproductive interests between the two levels are not aligned. In this thesis I explored this tension and the stabilising mechanisms that align the interests of different organisational levels at different levels of selection in the termite-fungus symbiosis.

The symbiosis between fungus-growing termites (Macrotermitinae) and Termitomyces fungi (Basidiomycota) evolved on time, approximately 30 million years ago, without subsequent reversals to non-symbiotic states. The symbiosis has often been described as a farming system in which the termite farmers cultivate their domesticated fungus. Over time both the termites and their fungi have become mutually and obligately dependent on each other, even though in most cases the termites and fungi have retained independent reproduction and dispersal. Independent reproduction implies that the reproductive interests of the termites and their symbionts are not completely aligned, leaving room for conflict between the partners. Since the symbiosis has remained stable over evolutionary time, it is likely that there are mechanisms that have stabilised this level of organisation.

One of the major questions in the termite-fungus symbiosis is how sexual reproduction in the partners is correlated in time. Even though the termites and their fungal symbionts reproduce and disperse independently to establish new colonies, the fungal symbiont typically forms mushrooms a few weeks after the colony has produced reproductive termites. It has been hypothesised that this timing of mushroom formation is due to a trade-off between alate and worker production by the queen of the termite colony. Under the assumption of a maximal rate of termite reproduction, investment in the production of alates leads to a reduction in the production of workers. Because workers consume the fungus, reduced numbers of workers will allow mushrooms to 'escape' from the host colony. In chapter 2 we tested a specific version of this hypothesis, viz. that the typical asexual structures found in all species of Termitomyces - nodules - are immature stages of mushrooms that are normally harvested in a primordial stage, except when there are too few workers. We refuted this version of the hypothesis by showing that nodules and mushrooms are completely different structures from the 
earliest developmental stages that we could sample. While our results indicate that a reduced number of workers is a necessary condition for the production of mushrooms, they also show that it is not a sufficient condition, and that other factors are also necessary to trigger the formation of mushrooms. In chapter 6 I discussed a possible mechanism that may trigger mushroom formation in Termitomyces fungi.

Due to the independent reproduction and dispersal of the termites and their fungi, the interaction between host and symbiont needs to be re-established at the start of each termite colony. It is known that there is a certain interaction specificity between termites and Termitomyces fungi, but unknown what factors contribute to the observed combinations of termite and fungus. It has been hypothesised that substrate provisioning by termite farmers could explain the observed interaction specificity. In chapter 3 we explored whether differences in nutrient requirement between fungi from different termite species can be found. We tested if differences in in vitro performance of Termitomyces cultivars from nests of three termite species on various substrates are correlated with the interaction specificity of their hosts. We showed that there were quantitative differences between biomass formation on different carbon sources and in a two-factor geometric framework experiment (simultaneously varying carbohydrate and protein availability), which indicates that substrate provisioning may contribute to selection of an adapted symbiont. However, future research needs to show whether those differences indeed contribute to selection of specific fungal cultivars by termites at the founding of a colony.

In the termite-fungus symbiosis, horizontal symbiont transmission is also associated with sexual reproduction of the fungus. The dispersing fungal spores are sexual spores produced in the mushrooms. It has been shown that for inhabitant symbionts, like Termitomyces, those that undergo little genetic change should be selected as they live in a stable biotic environment to which they have become adapted. Following from this observation, there should have been selection for a low recombination rate in Termitomyces fungi. In chapter 4 we constructed a new, more contiguous reference assembly of the Termitomyces symbiont of $M$. natalensis that allows for the study of recombinational landscapes. Also, we isolated a full-sibling mapping population of this Termitomyces species and used these to create the first linkage map of a Termitomyces fungus using a Genotyping-by-Sequencing approach. Finally, we performed an initial study into the recombination landscape of this Termitomyces species and showed that its recombination rate varies substantially across the genome. To be able to answer whether Termitomyces fungi indeed have evolved a low recombination rate, the recombination landscapes of more Termitomyces species as well as those of its close, free-living relatives should be studied.

In chapter 5 we zoomed in on the basidiomycete life cycle and explored how the peculiarities of basidiomycete life cycle open possibilities for lower-level selection that 
conflicts with the higher-level organisation (the fungal mycelium). The first difference between basidiomycetes and the vast majority of sexual life cycles is that after gamete fusion, the nuclei remain separate for almost the whole life cycle. The second difference is that the nuclei of two fusing gametes can move through the whole body - the whole mycelium - of their mating partner. We show that by remaining separate, the fates of these two separate nuclei are not fully aligned, which means that selection can act on the individual nuclei at the cost of the dikaryon. Also, we show that these life cycle peculiarities could enhance the conflict of interest between nuclei and mitochondria, possibly leading to reduced fitness of the dikaryon. In chapter 6 I elaborated on this chapter by discussing how the Termitomyces life cycle deviates from the 'standard' basidiomycete life cycle. Most Termitomyces species that were studied did not show nuclear migration, and do not have the typical clamp connections to ensure that only two nuclei (one of each mate) are present in each mycelial cell. I argue that absence of nuclear migration may reduce nuclear competition at the cost of the heterokaryon, but may enhance the conflict between nuclei and mitochondria through competition between the different parental mitochondria.

Although the stability of the termite-fungus symbiosis has attracted the interest of many evolutionary biologists, the interest in the termite-fungus symbiosis is not for fundamental questions only. All mushrooms of Termitomyces fungi are edible and considered delicacies in the areas where they are found. The work described in this thesis concerning Termitomyces will also aid the search for Termitomyces mushroom cultivation methods. The work in chapter 2 brings us closer to find the factors that promote mushroom formation. The work in chapter 3 will aid the optimisation of Termitomyces growth substrate. Finally, the work in chapter 4 could in the future help for breeding and analysing desirable traits for the cultivation of Termitomyces mushrooms, so that in future we will be able to re-domesticate the fungus that was domesticated by termites 30 million years ago. 


\section{Acknowledgements}

Analogously to raising children, I find, it takes a village to finish a $\mathrm{PhD}$ thesis. Here I would like to thank my 'village'.

First of all Wytske. I believe that I would have not even started this particular $\mathrm{PhD}$ if you had not told Duur I was looking for one. This is exemplary for your role within the group. You make sure that everyone stays connected and updated. Thank you for our Monday morning chats in the empty open office.

Professor Duur, you are a wonderful supervisor. Thank you for the support and freedom you have given me. With your calm, open and positive attitude you helped me take the edges of some of the harder things in the process. I think you are a great scientist and writer and I have learned lots from you. Bas, it was a pleasure to have you as a promotor and as a boss. I'm not sure whether you realise the importance of the 'assignment' you once gave me to figure out what I wanted to do after my $\mathrm{PhD}$. It paved the road to explore my possibilities and gave me the energy to finish my $\mathrm{PhD}$. It ultimately led to finding my new job and I am happy that in a sense you will continue to be my boss.

One of the reasons I wanted to do my $\mathrm{PhD}$ at the Laboratory of Genetics was the group-feeling that I had experienced during my BSc thesis. I was not let down. I enjoyed the coffee breaks, lunches, Christmas dinners, 'schoen zetten', Pub quizzes, Easter brunches, barbeques, lab-outings and all the songs that were written for celebrations. Thanks to all of you! My paranymph Margo, Kim and Anneloes, thank you for all the lovely discussions about science, life, and the open office space that was successfully made less open. Also, a special thanks to all (also former) people in 'group Duur' and to the (also former) technicians who make sure that our lab runs like a machine. Especially Marijke, who taught me all the ins and outs of culturing fungi. José, thank you for our early morning coffees sharing our lack of sleep. Fons, thank you for being like a surrogate supervisor. Your door was always open to discuss fungi, PFGE, recombination, weird genetic phenomena, education, science and the university policy.

A big thanks to all of the people at FABI who so generously hosted us for fieldwork; it was a pleasure meeting all of you. Bernard, a special thanks for sharing your childhood memories with me. I was moved by your immediate response, and your observation on how connected we all are. The field trips wouldn't have been half as much fun without the people that joined: it was great digging dirt, and dancing on the tables of the 'Springbok bar' with all of you.

A month or three into my $\mathrm{PhD}$, the first field trip was scheduled. I had an idea for what is now chapter 2 of this thesis, but I didn't know how to approach it. Norbert (Labora- 
tory of Cell Biology), thanks to you I knew what to collect and how to handle it. You made sure I came prepared and taught me everything I needed to know to get to the lovely pictures in the chapter. Patrick (Mushroom Group), you also helped making this experiment possible by lending me some material I was too late for ordering.

For chapter 3, Rafael visited me in Wageningen. We had a lot of fun taking over the lab with our crazy experiment. It was great having you as a writing buddy for this chapter. Thank you for sharing our PhDs and some wonderful times in Wageningen, South Africa, and Copenhagen. Peter and Joost, I was super happy that you wanted to be involved in chapter 4 . You both taught me a lot in a very short time, thank you for sharing your expertise. Ben and Kim, you also gave me some vital pointers for this chapter and Ben, thank you for regularly using your SUDO powers on Franklin for me.

Bas (Jacobs), Mandy, Nerissa, Lisa, Niko and Jan, thank you for having me as your supervisor. You did great work and I was happy to play a small part in your development. You'll find that all of your theses play some part in this thesis. Also a big thanks to the Mushroom Group, you have helped me out on multiple occasions with material and experience, you helped Jan with his experiments and we had some good discussions on the protein content of Termitomyces (Johan).

I would also like to thank Jessica Tummers, who helped me identify how I wanted to develop myself for my future career and pointed out that I should just start to express what I wanted. I took this advice and it was wonderful to see how many people wanted to help. With a special thanks to Lennart, Amber and Claudius, who gave me the opportunity to develop a pitching workshop. I am super happy that they will, together with Naomi, Inka, Anja, Theo, Anja, and Maartje, will be my new colleagues at PE\&RC.

All members of the WUR council, including Clem, Anneke, Hermijn and Monique, thank you very much for allowing me to be your chair. I learned a lot from each and every one of you. Thank you Marjan, for helping me get most out of this learning experience. Job, bad cop to my good cop, thanks for the fun, support and discussions.

Sara, thank you for planting the seed in me that it is actually possible to defend your thesis within contract time. I am proud that I managed to do it too.

Then, the non-work people who greatly contributed to my mental health and overall happiness. Lieve Berts, I love it that whenever we see each other we just pick up where we left off. Santé ermee. Lieve 'Bijklets date' thanks for 'het bijkletsen'. Lieve Bou en Benno, also known as Ome Boudie and Ome Benno, I am happy that being with Gerben meant that I got you as extra 'family'. Lieve Mir, thank you for sharing the good, the bad and the ugly of parenthood, and for bailing us out whenever Gerben and I have forgotten about Christmas dinners, birthdays, or Sinterklaas celebrations at day-care. 
Vera and Lenny, I loved and miss the wonderful hangout Thursdays in which we would not be able to finish a single sentence without being interrupted and yet manage to discuss some serious topics.

Technically, not really non-work at the beginning, but definitely non-work now, my fellow mum-of-two-and-PhD-candidate and paranymph Elysa. Thank you, for everything. We did it!

And then, all of a sudden, the pandemic happened. An interesting situation with two kids and two working parents at home. A big thanks to all of our 'achterpad' neighbours, you made this period much more enjoyable. You helped me push through, keep to the original deadline and maintain my sanity. Anita, thank you very much for painting the beautiful cover!

Last, but certainly not least, my family. Lieve papa en mama, bedankt dat jullie me altijd het vertrouwen hebben gegeven om mijn eigen keuzes te maken. Mama, bedankt voor je eindeloze geduld, nu ook met Luka en Imme, en bedankt voor het omdraaien van papa en mama in Jip en Janneke. Papa, ik heb genoten van de korte tijd dat we collega's waren. Bedankt voor het overdragen van jouw visie op onderwijs en onderzoek. Ik heb er veel aan gehad. En? Sta je nu je opa bent nog steeds achter stelling 12? Lieve Linda, thanks for being my sister. Ik heb grote bewondering voor hoe je je staande houdt, voor hoe je een prachtig mens bent geworden. Bedankt dat ik elke keer weer mag opscheppen over hoe de mooiste kleertjes van Luka en Imme door hun tante Linda zijn gemaakt! En dan zo goed als zusje, Steef en haar man Martin, bedankt voor alle All-You-NeedIs-Love kerstavonden. Een traditie die er wat mij betreft altijd in moet blijven.

Lieve Corrie en Bernard, ik kan me geen fijnere schoonouders wensen. Bedankt voor het warme welkom in jullie gezin en voor alle hulp. Lieve Geke en Karim, jullie ook bedankt voor jullie support! Zullen we snel weer een spelletje spelen?

Lieve, lieve Gerben, eigenlijk voelt alles wat ik hier op probeer te schrijven als een beetje afgezaagd. Ik ben gewoon ontzettend blij met je. Met je energie, je relativeringsvermogen, je optimisme, je begrip, je onvoorwaardelijke liefde en je bijdrage (zowel genetisch als opvoedkundig) aan onze zoons. Luka en Imme, lieve spiegels van me, bedankt voor alle lessen die jullie me nu al geleerd hebben.

I feel incredibly grateful to be surrounded by so many lovely people. 


\section{PE\&RC Training and Education Statement}

With the training and education activities listed below the $\mathrm{PhD}$ candidate has complied with the requirements set by the C.T. de Wit Graduate School for Production Ecology and Resource Conservation (PE\&RC) which comprises of a minimum total of 32 ECTS (= 22 weeks of activities)

\section{Review of literature (4.5 ECTS)}

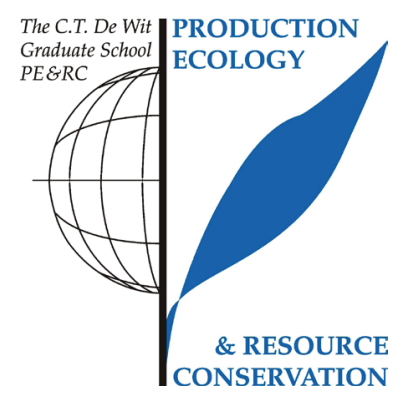

- Re-domesticating an ancient domesticated fungus

\section{Post-graduate courses (7.7 ECTS)}

- $\quad$ Systems biology: statistical analysis of omics data; EPS (2014)

- Evolutionary biology; Basel University \& ETH Zürich (2015)

- Life history theory; RUG (2015)

- $\quad$ Experimental design; WUR (2017)

\section{Laboratory training and working visits (2 ECTS)}

- Fungus growing termites and Termitomyces fieldwork and labwork in South Africa (2015)

Competence strengthening / skills courses (4.2 ECTS)

- $\quad$ Pitch training; In’to Languages (2015)

- Competence assessment; WGS (2015)

- $\quad$ Supervising students; WGS (2017)

- Science communication for Famelab winners; British Council (2017)

- Career assessment; PE\&RC (2017)

- Effective behaviour in you professional surroundings; PE\&RC (2018)

- $\quad$ Storytelling; In'to Languages (2018)

- Writing effective memo's; TAQT training (2018)

- Daily board training; Springest (2018)

- Personal coaching for chairing competencies; WUR (2018, 2019)

- $\quad$ Strategic behaviour and negotiation; TAQT training (2019)

PE\&RC Annual meetings, seminars and the PE\&RC weekend (1.5 ECTS)

- PE\&RC Day (2014)

- PE\&RC Workshop carousel $(2015,2018)$

- PE\&RC Last years weekend (2018) 
Discussion groups / local seminars / other scientific meetings (5.9 ECTS)

- WEES Seminars (2014-2019)

- Fall meeting of the KNVM division mycology (2015)

- B-wise Meeting (2016 - 2017)

- Microbial population biology (2016 - 2017)

- Distinguishing science and metaphysics in evolution and religion: Lorentz meeting (2018)

International symposia, workshops and conferences (9.6 ECTS)

- Seminar@ FABI for guests working on fungus growing termites including; oral presentation; Pretoria (2015)

- PhD Pitch symposium Dies Natalis,; oral presentation; Wageningen (2015)

- International Society for Mushroom Science; oral presentation; Amsterdam (2016)

- Famelab, local and national final; oral presentation; Wageningen, Utrecht (2017)

- ESEB; poster presentation; Groningen (2017)

Lecturing / supervision of practicals / tutorials (3 ECTS)

- Evolution and systematics (2015-2016)

- Genetic analysis, trends and concepts (2015-2017)

\section{Supervision of students (12 ECTS)}

- Genotyping-by-sequencing, linkage mapping, protoplasting, and mating of the Termitomyces symbiont of Macrotermes natalensis (MSc thesis)

- An analysis of substrate breakdown in Termitomyces sp. grown on various complex substrates (BSc thesis)

- Pulsed field gel electrophoresis of Termitomyces sp. to estimate chromosome number (BSc thesis)

- The effect of different nitrogen sources on the growth of 23 Termitomyces strains (BSc thesis)

- Nutrient requirements for Termitomyces species (MLO internship)

- Genetic transformation of Termitomyces associated with Macrotermes natalensis (MSc thesis) 
The research in this thesis was carried out at the Laboratory of Genetics at Wageningen University \& Research, the Netherlands. The work was financially supported by the Dutch Research Council (NWO, ALW Open Competition 824.01.002).

Cover art by Anita Stoof (anitaletuve.com)

Thesis layout by Sabine M.E. Vreeburg

Printed by DuurzameDissertatie.nl on 100\% recycled paper using water-based ink. A tree has been planted for every copy of this thesis. 